\title{
25th Congress of the European Society for Pediatric Neurosurgery (ESPN) Paris-France, 8-11 May 2016
}

(C) Springer-Verlag Berlin Heidelberg 2016

\section{Oral Presentations}

\section{Session 1: Spinal dysraphism}

\section{OP01}

\section{Gait changes in children with spinal lipoma}

Lucy Alderson, Lindy May, Christina Rampota, Dominic Thompson (London, United Kingdom)

Objective: To describe gait of children with lipoma in a clinical setting. Children with lipomyelomeningocele (spinal lipo$\mathrm{ma}$ ) have a spectrum of physical presentations affecting neurological and urological function, musculoskeletal deformity, pain and motor deficits (Kanev et al., 1990; Kulwin et al, 2013; Segal et al, 2013). Evidence to support prophylactic untethering is conflicting. Current practice at GOSH is to monitor within an MDT clinic and intervene promptly if function deteriorates. Identifying deterioration is challenging as baseline function and tethering presentations vary. Standard mobility classification is often insensitive to symptom progression. This study explores identification of subtle walking deficits in this population, and monitoring progress or progression of symptoms.

Material and Methods: 26 children who attended dysraphic clinic at GOSH between Jan 2013-June 2014 completed a GAITRite assessment as part of routine follow up. Children walked across a $6 \mathrm{~m}$ pressure sensitive carpet 3 times at preferred, slow and fast speeds. 2 children were assessed at multiple time intervals. Approval for retrospective review was granted by GOSH/ICH Research and Development Office.

Results: Mean age was 8.6 years (range 3.8-17.9). Preferred velocity mean (sd) was $116.2 \mathrm{~cm} / \mathrm{s}(21.0)$; fast velocity 172.0 $\mathrm{cm} / \mathrm{s}$ (37.3). Data was plotted on normal gait centiles, from 650 typically developing children, and converted to $\mathrm{Z}$ scores using LMS-Growth. Preferred walking velocity fell within the
25 th and 75 th centiles with no significant difference between $\mathrm{Z}$ scores and predicted age means ( $\mathrm{p}>0.05)$. Velocity, cadence ( $p<0.01$ ), single support and double support $(p=0.02)$ were all significantly different for the lipoma group at fast speeds.

Conclusion: Velocity of children with lipoma falls within normal ranges at preferred speed. Progressive symptoms are associated with reduced endurance, falling behind peers, with slowing of gait when walking fast. Longitudinal gait measurement in children with Lipoma at fast and preferred speeds may identify subtle deteriorations, aiding surgical decision making.

\section{OP02}

Spinal lipoma of the filum terminale: review of 174 consecutive patients

Kenichi Usami, Pauline Lallemant, Thomas Roujeau, Syril James, Kevin Beccaria, Raphael Levy, Christian Sainte-Rose, Michel Zerah (Paris, France)

Purpose: Spinal lipoma of the filum terminale (LFT) is a congenital lumbosacral anomaly that can cause tethered cord syndrome. Purposes of this study are to clarify preoperative characteristics of LFT, to elucidate surgical effects, and to discuss the reasonability of prophylactic surgery for LFT.

Methods: One hundred and seventy four children (20082014) who underwent section of LFT were prospectively followed for prevalence of symptoms, skin stigmas, and associated malformations, motivator of diagnosis, conus level, and surgical outcome. Mean age at surgery was $4.1 \pm 4.2$ years old (37 days to 17.7 years).

Results: Ninety-four children (54.0\%) had skin stigmas and $60(34.5 \%)$ had certain perineal malformations. Seventy-nine children (45.4\%) were symptomatic. The most common motivator for diagnosis was skin stigmas $(44.3 \%)$, followed by syndromes $(33.3 \%)$, and symptoms $(20.1 \%)$. The age at surgery was significantly older in symptomatic patients than in 
asymptomatic patients $(\mathrm{p}<0.001)$. Surgery improved symptoms in $50 \%$ of patients with 2.1 years follow-up period. Of 85 asymptomatic patients, all except one remained asymptomatic postoperatively and none of the symptomatic patients deteriorated. The presence of associated malformations and the conus level did not affect surgical outcome. Postoperative complications developed in nine patients (5.2\%): 7 transient local problems, 1 definitive urological deterioration, and 1 transient respiratory problem.

Conclusion: Surgery for LFT was a simple and safe procedure. It improved half of the symptomatic patients and stopped the deterioration of the others. Even if only one of the asymptomatic patients deteriorated at maximum of follow-up, the role of prophylactic surgery remains a point of discussion.

\section{OP03}

Surgical treatment in children spinal cord lipomas with tethered cord syndrome

Ai-Jia Shang (Beijing, China)

Objective: To explore the surgical treatment in children with tethered cord syndrome (TCS) of lipomatous type and analyze the curative effect of it.

Methods: According to MRI performance, 275 cases of children with TCS of lipomatous type were classified into four types, namely dorsal type, caudal type, chaotic type, lipomyelomeningocele type. All these cases were being treated by microscopic surgeries. Operative effect and prognosis of different types of lipomatous TCS were analyzed.

Results: 275 children were being followed up for 6 to 60 months with an average of 20 months. The curative effects were evaluated by Spina Bifida Neurological Scale (SBNS) which is based on function, reflex, bladder and bowel function of sensory and motor. Factors that affect surgical treatment and prognosis were analyzed. The result indicates that the total effective rate of postoperative children with lipomatous TCS was $76 \%$ in this group. Different types of efficiency were as follows. Best results were obtained in dorsal type cases, following by caudal type, lipo-myelomeningocele type and chaotic type. Total resection and near-total resection are better than partial resection.

Conclusions: Once diagnosis is confirmed, early microsurgical operation should be taken in lipomatous TCS cases. Early surgical treatment can prevent or release the dysfunction of bladder, bowel and lower limb. To get total resection or near-total resection of the lipomas and complete release of spinal cord and cauda equina, surgery should be guided by electrophysiology. Reconstruction of neural placode and prevention of adhesion should be achieved as well. The operation effect and prognosis are mainly related to lipomas types, timing of operation, severity of symptoms, thorough release of nerves can be achieved or not, and retethering.

\section{OP04}

Hemimyelomeningocele: clinico-pathological considerations Memet M. Ozek, Murat Başarır (Istanbul, Turkey)

Objective: The term hemimyelomeningocele refers to a rare condition of spinal dysraphism in which open neural placode is localized in one hemicord of a split cord malformation of type I. This entity requieres attention due to lack of overt clinical evidence and neuroradiological investigation since the open neural tube defect of the newborn is generally repaired as an emergency.

Method: Between 1989 and 2013800 newborns and infants with an open neural tube defect were operated on at the Division of Pediatric Neurosurgery at Marmara University Medical Center and Acıbadem University, Department of Neurosurgery. 168 patients (21\%) presented an associated split cord malformation and within this group 32 cases (4\%) were diagnosed as hemimyelomeningocele. The diagnosis of hemimyelomeningocele have been made either during preoperative physical examination or incidentally during operation. In 28 cases the placode had a paramedian localization, with hypertrichosis in 16 cases. Scoliosis was present in 11 cases with an additional foot deformity in 6 of them. In cases with neurological deficit, the deficit was typically asymmetrical. Five cases have been noted to have a normal neurological examination. Sensorimotor function was preserved on the side of the closed hemicord. In surgery, the neural tube is reconstructed, the septum removed, and a duraplasty was performed. There were no surgical morbidity and mortality. Detailed postoperative investigation with magnetic resonance imaging revealed the split cord malformation with two hemicords. Within the first month after surgery urine analysis \& culture, urinary system $\mathrm{U} / \mathrm{S}$ and voiding cystouretrogram were also performed.

Results: Only two patients presented with neurogenic bladder in the long-term follow-up period. The mean follow-up period was 5 years. Nine children needed a shunt during early infancy. Twelve children were classified as Gr. 2, whereas twenty as Gr. 3 according to the Oi and Matsumato's Spina Bifida Neurological Scale (SBNS).

Conclusions: Hemimyelomeningocele is a rare subgroup of Spina bifida, which has significantly better prognosis compared to other types of Spina bifida. Preoperative diagnosis of both hemicords is crucial to preserve the intact hemicord during surgery.

\section{OP05}

Closure of myelomeningocele. Does timing matter?

Tafadzwa Mandiwanza, Samuel Ndoro, John Caird, Darach Crimmins (Dublin, Ireland)

Introduction: Myelomenigocele (MMC) remains prevalent in Ireland with 25 to 30 new cases per year. Standard neurosurgical 
management includes early closure of the defect and consideration of CSF diversion. International best practice suggests closure of the defect should be performed within 48 hours of birth to prevent CSF leakage, infection and for ease of care.

Aims: We wished to examine timing of closure in our children with MMC and how it relates to incidence of wound problems, rates of meningitis and shunt infections.

Methods: Retrospective review of all newborn children in the Irish Republic with MMC from 2009 to 2014. They were divided into two groups: early closure (within 48 hours) and delayed closure (after 48 hours).

Results: 141 children were born with MMC in Ireland during this period. Eighty-three (59\%) were female. Seventy $(50 \%)$ of children had their myelomeningocele closed within 48 hours. Of the 70 cases in the early closure group, 46 required a CSF shunt (66\%). Twenty seven $(39 \%)$ of the early closure group had complex procedures (rotational flaps). In the early closure group $5 / 46$ shunts became infected (11\%). There were 5/70 wound breakdowns/CSF leaks (7\%) and 5/70 children developed meningitis $(7 \%)$. There were 68 cases in the delayed closure group 58 of whom required a CSF shunt $(85 \%)$. Twenty nine $(43 \%)$ of the delayed closure group had complex procedures (rotational flaps). In the delayed closure group 10/58 shunts became infected (22\%). There were 10/68 wound breakdowns/CSF leaks $(15 \%)$ and $10 / 68$ children developed meningitis (15\%). Three children had no surgery for their lesion. One with multiple anomalies died early in the neonatal period following CSF shunting. One had an partially skin covered sacral lesion that never leaked (shunted for hydrocephalus). The third had a large thoracic lesion that epithelialized spontaneously without the need for a shunt. Altogether 106 children required CSF shunts. Five shunts were placed before closure with 2 becoming infected (40\%). Eleven shunts were placed simultaneous to closure with 1 infection (9\%). Eighty-eight shunts were inserted after closure with 15 becoming infected $(17 \%)$. Shunt infection was more likely in cases of wound leak (27\%) than without wound leak (16\%). Meningitis was also more common in cases of wound leak $(27 \%)$ than without a leak $(9 \%)$. Two children had shunts and were never closed.

Conclusions: There is a higher rate of meningitis and shunt infection with delayed myelomeningocele closure and where there were post-operative wound problems. We would therefore advocate myelomeningocele closure within 48 hours of birth, careful wound vigilance and simultaneous shunt or EVD placement where there is significant hydrocephalus and likelihood of wound problems.

\section{OP06}

Cellular and molecular lesion mechanisms in the human myelomeningocele placode

Friederike Knerlich-Lukoschus, Bea Kowitzke, Ivo Leuschner, Arend Koch, Michael Synowitz, Janka Held-Feindt, Gesa Cohrs (Kiel, Germany; Berlin, Germany)

Introduction: Myelomeningocele (MMC) repair primarily addresses the deficient neurulation defect. Assuming that traumatic insults on the exposed placodes induce further lesion-amplifying cascades the aim of this study was to identify underlying cellular and molecular changes, which might provide targets for novel adjuvant treatment strategies.

Material and Methods: Ethical board approval was obtained. 17 specimens from MMC-repair surgeries performed within 72 hours after birth, were identified that harbored sufficient neuroepithelial tissue for further investigations. Respective clinical data were collected. Controls included normal adult and fetal spinal cord (sc) specimens. Sections were immunostained with neuroglial, neural crest, mesenchymal and epithelial marker antibodies, and CD3 as inflammatory marker. Immunohistochemistry for Interleukin-1beta/-receptor (IL-1b/ IL-1R1), erythropoietin/-receptor (EPO/EPO-R) and Hypoxia Inducible Factor (HIF) 1 and 2 was performed and analyzed qualitatively and semiquantitatively (ImageJ $1.43 \mathrm{u}, \mathrm{NIH}, \mathrm{USA}$ ). Cellular localization was confirmed by double-fluorescence-labeling with respective cellular markers. Cytokine- and GFAP expression was further analyzed on mRNA level by real-time RTPCR.

Results: Investigated placodes exhibited comparable cellular profiles with consistent neuronal and epithelial cellular marker expression. All placodes presented with significantly elevated GFAP-, prominent Vimentin-, and partially CD3-immunoreactivity. All cases exhibited significant multi-cellular induction of IL-1beta and IL1-R1 ( $\mathrm{p} \leq 0.001$ vs. control). EPOR immunoreactivity was significantly elevated in most cases with neuronal expression pattern, while its ligand was expressed more inconsistently and co-stained with neuronal and microglial markers. EPO also co-stained with HIF-2alpha, which, in contrary to HIF-1alpha, was induced significantly in all cases. Real-time RT-PCR confirmed GFAP and cytokine induction on mRNA level.

Conclusion: These studies confirmed induction of cellular and molecular changes in the human MMC placodes that resemble secondary lesions cascades induced by sc trauma. For the first time pro-inflammatory and neuroprotective cytokine expression was demonstrated in MMC placodes, providing new potential targets for adjuvant treatment regimens of open neural tube defects. 
Session 2: Advances in pediatric head trauma management

\section{OP07}

Surgical options for treatment of traumatic subdural hematomas in children with suspected abusive head trauma

José Roberto Tude Melo, Federico Di Rocco, Christian Sainte-Rose, Philippe Meyer, Michel Zerah (Paris, France; Salvador de Bahia, Brazil)

Introduction: Subdural hematoma (SDH) is the most common finding in children victim of abusive head trauma (AHT) during the first year of life. Our scope is to report our experience, and to discuss the routine surgical options in SDH, managed at a Level 1 Pediatric Trauma Center.

Methods: A cross-sectional study with a consecutive revision of data described in medical records, of patients at risk of having AHT, between January 2008 and June 2013, in Ile de France. The inclusion criteria were the absence of a clear witnessed history of accident and a SDH identified at CT-scan. This single center study was in agreement with French law requirements.

Results: 184 children less than 2 years of age with SDH were included. Median age was 5.8 months, with a male predominance $(70 \%)$. A neurosurgical treatment was necessary in 111 children (60\%) with Glasgow Coma Score $\leq 12$, bulging fontanels or other suggestive signs of intracranial hypertension. The first surgical option was a subdural peritoneal shunt in 48\% (53/ 111), a subdural subgaleal shunt in 17\% (19/111), external subdural drainage in 16\% (18/111), transcutaneous subdural puncture in $15 \%(17 / 111)$, a craniotomy in $1.8 \%(2 / 111)$, and a decompressive craniectomy in $1.8 \%(2 / 111)$. We verified that in $82 \%$ of the children who benefited of a transcutaneous subdural puncture, and in $33 \%$ of those with an external subdural drainage, an increased or persistancy of the SDH were observed and further surgical intervention was required.

Conclusions: The choice of SDH treatment should be focused on each case, according to the clinical and radiological presentation. Although effective on an emergency basis, transcutaneous subdural puncture and external subdural drainage may be insufficient to obtain complete resolution of SDH, and a transient subdural peritoneal shunt may be needed.

\section{OP08}

Continuous multimodality monitoring in children after traumatic brain injury - Preliminary experience

Adam Young, Joseph Donnelly, Marek Czosnyka, Ibrahim Jalloh, Xiuyun Liu, Marcel Aries, Helen Fernandes, Mathew Garnett, Peter Smielewski, Peter Hutchinson, Shruti Agrawal (Cambridge, United Kingdom)

Introduction: Multimodality monitoring is regularly employed in comatose adult traumatic brain injury (TBI) patients and has provided crucial insight into the pathophysiology and patient management of this heterogeneous condition. Pediatric studies are less frequent.

Methods: An analysis of data collected prospectively from 12 pediatric TBI patients admitted to the Addenbrooke's Hospital Pediatric Intensive Care Unit (PICU) between August 2012 and December 2014 was performed. Patients' intracranial pressure (ICP), arterial blood pressure (ABP), and cerebral perfusion pressure (CPP) were monitored continuously using brain monitoring software $\mathrm{ICM}^{+\mathbb{B}}$ ) Pressure reactivity index $(\mathrm{PRx})$ and "Optimal CPP" (CPPopt) were calculated. Patient outcome was dichotomized into survivors and non-survivors.

Results: At 6 months 8/12 (66\%) of the cohort survived the TBI. The median $( \pm \mathrm{IQR})$ ICP was significantly lower in survivors $13.1 \pm 3.2 \mathrm{~mm} \mathrm{Hg}$ compared to non-survivors $(21.6 \pm 42.9 \mathrm{~mm}$ $\mathrm{Hg}$; $\mathrm{p}=0.003$ ). The time spent with ICP over $20 \mathrm{~mm} \mathrm{Hg}$ was lower in survivors $(9.7 \pm 9.8 \%)$ vs $60.5 \pm 67.4 \%$ in non-survivors; $\mathrm{p}=0.003$ ). Although there was no evidence that CPP was different between survival groups, the time spent with a CPP close (within $10 \mathrm{~mm} \mathrm{Hg}$ ) to the optimal CPP was significantly longer in survivors $(90.7 \pm 12.6 \%)$ compared with non-survivors (70.6 $\pm 21.8 \%$; $\mathrm{p}=0.02$ ). The pressure reactivity index ( $\mathrm{PRx}$ ) provided significant outcome separation with survivors measured at 0.02 \pm 0.19 compared to non-survivors at $0.39 \pm 0.33(\mathrm{p}=0.02)$.

Conclusion: Our observations provide evidence that multimodality monitoring may be useful in pediatric TBI with ICP, deviation of CPP from CPPopt, and PRx being strong determinants of outcome.

\section{OP09}

"Wait and see" policy as the first-line management of radiologically significant epidural hematomas in pediatric patients with normal initial neurological examination. A 3-years experience of our department

Georgios Markogiannakis, Olympia Karypidou, Dimitris Panagopoulos, Niki Pavlatou, Michael Koutzoglou (Athens, Greece)

Introduction: Traumatic epidural hematomas (EDH) with significant radiological size are traditionally treated with craniotomy and evacuation of the epidural blood clot. At the same time, in children, a normal initial neurological assessment may indicate a conservative "wait and see" approach, a strategy recently employed by many pediatric Neurosurgical centers and reported in the literature. We report our 3 year experience in non-operative management of traumatic EDH analyzing clinical and radiological parameters.

Methods: We retrospectively reviewed a series of 6 patients who were conservatively treated with serial neurological examinations during 2012-2015. Inclusion criteria were an initial GCS score of 15, absence of neurological deficit, supratentorial location of hematoma and thickness $>1 \mathrm{~cm}$. All patients were initially applied a rigorous neurological 
assessment protocol that was succeeded by a clinical and radiological follow-up surveillance.

Results: 6 children ( 5 boys, 1 girl) with supratentorial EDH met the inclusion criteria of our study and all of them were managed non-operatively. Mechanism of injury, time until EDH diagnosis, localization of hematoma and size on axial slices and associated symptoms of increased intracranial pressure (headache, vomiting) were studied.

No post-traumatic sequelae were recorded; all patients were seizure free and had an uncomplicated course at follow-up reexaminations.

Conclusion: Our results are in accordance with a recently emerged body of literature which supports the conservative management of significant size EDH in the pediatric population under strict clinical standards. Factors that corroborate this policy in children are the usual venous origin of EDH and their potential to tolerate better an increase of intracranial pressure. Essential prerequisites are the ability to perform careful serial neurological observation under a standardized protocol and the capacity of the centre facilities to provide immediate hematoma evacuation in case of acute neurological deterioration.

\section{Session 3: Spine}

\section{OP10}

The risk of meningitis in spinal dermal sinuses: when, not if Matthieu Vinchon, Irene Stella (Lille, France)

Dermal sinuses carry a risk of infection and require prophylactic surgery. However, the delay of infection is not well known, and appears markedly different for cranial and for spinal dermal sinuses (SDS). As a result, the ideal timing of surgery for SDS is not established.

We reviewed cases of SDS treated in our institution since 1987. During that period, our general policy has been to operate children before the age of 6 months whenever possible. We compared infected versus non-infected cases.

We operated 31 children for SDS; the median age at surgery was 10 months. Sixteen of these $(52 \%)$ were infected at the time of diagnosis. The cumulated incidence of infection was $12 \%$ at 12 months, and $50 \%$ at 2 years. The mean followup was 72 months. The outcome regarding development, sphincter control and spinal deformity was poorer in the infected group, although the figures did not reach statistical significance. All patients with severe sequelae (dependency for daily care) were in the infected group.

The risk of meningeal infection is very high in SDS. Furthermore, infection often occurs during the first months of life, earlier than in cranial dermal sinuses. In our experience, infection had dire consequences on long-term outcome. SDS should be looked for systematically at birth and operated prophylactically within the first month of life whenever possible.

\section{OP11}

Craniovertebral junction fixation in children under 5

Patrick Grover, Dominic Thompson (London, United Kingdom)

Introduction: Rigid internal fixation for the treatment of paediatric craniovertebral (CVJ) instability has been shown to be safe and effective. However in young children skeletal immaturity, bone dysplasia and congenital anomalies of development frequently preclude standard instrumentation. We report a single centre experience of CVJ stabilization in children under 5 years old.

Methods: Children under 5 years undergoing internal fixation procedures for CVJ instability were reviewed. Primary diagnosis, indication for surgery, pre-operative neurological status extent of fixation and operative technique was recorded. Primary outcome measures were radiological fusion and neurological status at follow up. Secondary outcomes were procedure related morbidity and need for second surgery.

Results: 28 cases were identified, median age 2.3 years (range 9 -59 months). Diagnoses comprised; bone dysplasia (13), nonsyndromic CVJ anomaly (6), trauma (5), tumour (2) and infection (2). There were 21 occipitocervical and $7 \mathrm{C} 1 / 2$ fixations.

Mode of fixation was a) Calvarial autologous bone graft secured with cables (20) b) Occipital plate and rod construct with cables (6) and c) Rigid cervical screw and occipital plate fixation (1).

Median follow up was 2.6 years. 23/28 (83\%) achieved fusion after initial surgery. 2 cases required revision due to construct failure. $93 \%$ of patients had stable or improved neurology post-operatively. Two patients had post-operative neurological deterioration. There was no procedure related mortality. 4 patients were dead at latest follow up due to tumour progression (2), persisting bulbar compromise (2). Pin site infections were common (5).

Conclusion: For young children, high fusion rates with good outcomes can be achieved using semi-rigid constructs. However, these techniques require a period of external immobilization with halo-body orthosis entailing additional anaesthetic procedures. Whilst the authors recognise the need to embrace and refine internal fixation technologies for younger children the strategy proposed here has shown to be effective for both radiological and clinical outcome.

\section{OP12}

Central sleep apneas: related symptom or criterion of effectiveness after craniovertebral junction anomalies (CVJA) surgery?

Alice Rolland, Régis Lopez, Emilie Chan Seng, Maxime Ros, Yves Dauvilliers, Thomas Roujeau (Montpellier, France)

Introduction: Not all related symptoms improve after Chiari surgery. In some cases, effectiveness of 
decompression is difficult to ascertain and second look surgery can be discussed. We hypothesized that Central sleep apneas (CSA) could be an objective criterion of efficient hindbrain decompression.

Methods: We prospectively studied 44 consecutive pediatric cranio vertebral junction anomalies between 2012 and 2015 in our center (16 girls, 28 boys, median age 10.4 years old). All patients had videopolysomnographic evaluation. CSA were defined by an apnea-hypopnea index superior than 1 event per hour. Symptomatic patients were operated while asymptomatic ones were followed. Postoperative polysomnography was performed when preoperatively abnormal. We compared symptomatic patients according to presence or absence of preoperative CSA.

Results: 30 patients were followed and 14 were operated, (11 Chiari, 1 complex CVJA, 1 achondroplasy, 2 occipito-cervical malformations). 2 patients were operated twice.

No CSA was found among the 30 asymptomatic patients but in $8 / 14$ of the symptomatic group. Clinical symptoms and CSA were improved in all cases after surgery.

Incomplete improvement led to second surgery in 2 cases in which syrinx and residual CSA resolved after redo.

In the symptomatic group with CSA, we encountered 2 syrinx and 1 posterior fossa cyst. Among the symptomatic patients without CSA, there were 2 syrinx, 1 hydrocephalus.

Conclusion: Central sleep apneas seem to be related to symptomatic CVJA in accordance with previous studies.

CSA seem to be improved by surgery which has not been previously published. Moreover, when incomplete the improvement is after a first surgery, it disappears when redo.

Further studies are needed to confirm that polysomnographic anomalies by themselves could indicate surgery, or that incomplete cure of CSA should indicate redo.

\section{OP13}

\section{Reversed rotatory atlanto-axial subluxations: proposing a} new entity

Sandip Chatterjee (Kolkata, India)

Introduction: Anomalies at the craniovertebral junction need to be evaluated on the basis of their embryological development. This is a review of 6 cases of torticollis with "reverse" rotatory atlantoaxial subluxations occurring as a compensatory measure to a lateral tilt produced by nondevelopment of the lateral mass of atlas/occiput on one side as a failure of the development of the lateral proatlas elements. Here the axis rotates round a fixed atlas as a compensatory mechanism, hence called "reversed" atlantoaxial rotatory subluxation.

Aim of the Study: To introduce the concept of "reverse" rotatory atlanto-axial fixation (AARF) in the setting of congenital maldevelopment of the lateral elements of the proatlas.
Material and Methods: We encountered 6 cases of this anomaly. 4 of these presented with progressive torticollis and neurodeficit within the first 5 years of life, and the other 2 had symptoms after trivial trauma. In all cases, CT scans documented fixed atlas with defect in lateral mass of atlas and condyle of occiput on the same side, with rotation of the axis on the fixed atlas.

Results: The first 3 patients underwent occipito-cervical fusion. In all 3 cases, paraparesis improved after surgery. In 2 cases, opening up and fusion of the atlanto-axial joint on both sides, and in one case atlantoaxial distraction was produced using laminar hooks on the posterior arch of the atlas with transpedicular screws into the axis. In all cases the torticollis improved after surgery.

Conclusion: It is necessary to evaluate the exact embryological defect in children presenting with torticollis, and to tailor treatment strategy according to the anatomical deficiency causing the neurological problem. The unifying factor in all these 4 children has been the maldevelopment of the lateral sclerotome of the proatlas with or without accompanying maldevelopment of the $\mathrm{C} 1$ segment, resulting in the "reverse" rotatory subluxation.

\section{OP14}

Do we fix, halo or collar pediatric atlanto-axial rotatory fixation (AARF)? Is less more?!

Desedario Rodrigues, Digant Kamdar, Guirish A. Solanki (Birmingham, United Kingdom)

Introduction: While torticollis is commonly seen, true AARF cock-robin deformity is an uncommon paediatric injury with potential for severe neurological deficit. Some experts use traction (up-to $72 \mathrm{hrs)}$ ) to determine treatment (orthosis or surgery). However very young age, learning disabilities, autistic spectrum behaviour or simply children too frightened to cooperate makes treatment fraught with difficulties. We report our institutional experience in the management of AARF.

Patients: 32 children (19 girls, 13 boys) were treated between 2007 and 2015. F:M ratio:1.46. Median age: 6.4 years. Girls significantly older ( $81 / 2$ vs. $53 / 4$ ) years.

Methods: Fielding's radiological classification was used. Only children with radiologically confirmed AARF were included. Children were treated with muscle relaxation, neck collar and manipulated under anaesthetic (MUA) at the earliest opportunity. Halo orthosis (Halo) used in late ( $>1$ month) presentation or failure to reduce. Surgery for recurrence despite Halo immobilisation.

Results: Falls and trauma accounted for $53 \%(37.5 \%, 15.6 \%)$ of cases respectively. $25 \%$ (8) were due to post-infectious syndrome (Grisel's, TB osteomyelitis), 9\% (3) in Down's, $6 \%$ (2) were iatrogenic following surgery, $6 \%$ (2) idiopathic cases. Post-Infectious cases were three times higher in girls. $62.5 \%$ (20) children were initially treated with a collar. $66 \%$ 
(21) ultimately required a halo orthosis. TB osteomyelitis required prolonged antibiotic treatment and halo immobilisation. However, only one child $(3 \%)$ required a posterior C12 fusion for recurrent AARF. Complications included pin-site infection and halo slippage. There were no treatment-related cranial nerve or neurological deficits.

Conclusions: In children, AARF presenting with a painful torticollis often resolves with muscle relaxation and neck collar alone over a period of days. We propose that early presentation should be treated with muscle relaxants and a wellfitting collar initially \pm MUA. Halo should be considered if MUA and collar treatment fail, or in overt instability. While rare, instability or recurrent AARF (despite Halo immobilisation over 3 months) should be considered for surgery.

\section{OP15}

Foramen magnum stenosis in achondroplasia: management roadmap

Armando Cama, Gianluca Piatelli, Marcello Ravegnani, Alessandro Consales, Marco Pavanello, Paola Lanteri, Andrea Rossi (Genova, Italy)

Introduction: Achondroplasia is the most common form of skeletal dysplasia that requires spinal surgery in the pediatric age group. Foramen magnum stenosis is its most severe pathological feature, and may cause hindbrain dysfunction. We aim to present our single-centre experience in the neurosurgical management of achondroplasic infants.

Material and Methods: We retrospectively analysed a group of 90 achondroplastic infants (age range: 3-40 months; median, 18 months) who underwent occipito-cervical decompression for foramen magnum stenosis. We reviewed the clinical, neuroradiological and neurophysiological features, the surgical strategy, and the outcome. All patients presented with progressive neurological deterioration and/or neurophysiological alteration. With a standard neuroradiological planning (plain and dynamic radiographs, 3D CT scan, cerebral and cervical MRI with MR angiography) we assessed the morphology of the foramen and of the nervous tissues, the stability of the cranio-cervical junction (CCJ), and associated cerebral pathologies and vascular abnormalities. Neurophysiological studies were performed to quantify the neurological damage in sympomatic patients and to assess early neurological deterioration also in asymptomatic patients.

Results: Perioperative mortality was $0 \%$. There was 1 case of severe broncopneumonitis and another case of respiratory distress with gastroesophagitis and iliac thrombosis, which completely recovered. At follow-up (range 6-48 months) we observed neurological improvement in $90 \%$ of cases and neurophysiological improvement in $95 \%$. Two children with severe neurological status (tetraparesis, recurrent apnea) before surgery had a long postoperative period of clinical stability but died for respiratory complications and pneumonia at 2 and 4 years, respectively.
Discussion: Based on our experience, we propose a protocol for the treatment of foramen magnum stenosis in achondroplastic infants: 1) within the first year of life: clinical, neuroradiological, and neurophysiological assessment; 2 ) if the first screening is normal, continue to follow-up with a new MRI every 3 years; 3 ) craniocervical decompression for foramen magnum stenosis if clinical and/or neuroradiological and/or neurophisiological signs are present; 4) surgical procedure is supported by fibroscopic intubation and neurophysiological monitoring (SSEP); 5) post-operative soft collar is kept in place for 3 months; 6) a multidisciplinary follow-up is planned.

This multidisciplinary approach should include asymptomatic infants, as early diagnosis of foramen magnum stenosis, with early decompression whenever necessary, may improve surgical and neurological outcome and prevent clinical deterioration or sudden death.

\section{Session 4: Pediatric Neurosurgery and Technology}

\section{OP16}

Combining fMRI, MRI tractography and TMS with intraoperative electrophysiology and imaging to maximize radicality as well as functional preservation in eloquent area surgery

Martin U. Schuhmann, Marina Liebsch, Teresa Leao Tatagiba, Constantin Roder, Sotirios Bisdas, Samuel Gröschel, Marco Wilke (Tuebingen, Germany)

Introduction: Extensive functional MRI, tractography and TMS are rarely used in children for the pre-operative workup due to a presumed age related inability of children to perform accordingly in the scanner. We have shown that a dedicated team ín an appropriate environment is able to establish long imaging in awake children. This information, used for meticulous planning of surgical strategy, needs to be combined with intraoperative monitoring and imaging to optimize radicality and functional preservation.

Methods: 21 pediatric patients were included with either low grad glioma $(\mathrm{n}=14)$ or cavernomas $(\mathrm{n}=7)$ of central region, (left) temporal (region) or midbrainThey received: fMRI for language detection with up to 4 paradigms $(n=10)$, fMRI of motor cortex $(n=11)$, tractography of motor tract $(n=13)$, tractography visual tract $(n=9)$ and arcuate fascicle $(n=3)$. TMS was performed in 5 cases. High resolution intraoperative ultrasound was used in 19 operations and iMRI in 9.

Results: Desired functional or tract information was obtained in all cases. Surgical corridors were defined according to those results. In one case family decided against surgery, in all other results helped to balance risk. Extended intra-operative neuromontoring was used to confirm pre-operatively defined functionality if ever possible. On child underwent awake surgery because of arcuate fascicle involvement. Surgical goals 
according to intention to treat, which was mostly radical resection, could be achieved in all cases. There were no permanent new functional deficits except for partial loss of visual field in 1 case. Intraoperative ultrasound was most helpful, supplemented by iMRI, to achieve radicality.

Conclusions: Modern functional MRI and tractography are possible in awake children form 5 years onwards if an adequate study atmosphere is maintained. Tractography can be done for visual tract and arcuate fascicle equally good as motor tractography. In combination with intraoperative neuromonitoring, high-resolution ultrasound and iMRI, this information enables the pediatric neurosurgeon to successfully "walk" on the thin red line between radicality and functional impairment without harming. Thus these time consuming additional techniques should be used whenever possible to make surgery of lesions in eloquent areas as safe as possible and thus rewarding for surgeon and patient.

\section{OP17}

Lessons learned with the intraoperative magnetic resonance in a pediatric unit after 5 years

Sonia Tejada, Benedetta Pettorini, Chris Parks, Santosh Mohan Rao Kanangi, Shivram Avula, Joszef Lang, Dawn Hennigan, Sasha Burn, Ajay Sihna, Conor Mallucci (Pamplona, Spain; Liverpool, United Kingdom)

Introduction: The aims of this communication are to report our experience with the intraoperative magnetic resonance (ioMRI) in brain surgery, exclusively in pediatric patients as a routinely instrument since 2009 and discuss its potential clinical benefits in paediatric neurosurgery.

Methods: All the data has been collected prospectively from the database built with the surgical, radiological reports and clinical notes. The MRI findings were recorded in the radiological report, and the final decision was registered in the surgical notes. We have compared our data with those published in the literature.

Results: The ioMRI was introduced in our unit in 2009, since then, 255 cases have undergone surgery within intraoperative images. The mean age was 9.4 years (range 1 month-21 years), and the male/female ratio was 1.36/1. The most frequent type of lesion treated under ioMR were tumours, 152 were low grade tumours (WHO grade I and II) vs 64 high grade (WHO grade III and IV). 39 were non-tumour diseases. In 172 patients no actions followed the ioMR (67.4\%) because the neurosurgeon had achieved the first aim of the surgery. When complete resection was the aim, 32 out of 116 patients went for further resection after the ioMRI $(27.5 \%)$, whereas when the aim was debulking the tumour, 33 out of $59(56 \%)$ had more resection. The reoperation rate was $0 \%$ at 6 months. The mean duration of the MR was 31 minutes (5-45 min). There were no issues related with the head holder, the pins, and with the patient position. No incidents regarding excessive heating have been noticed.
Conclusions: This is the largest pediatric series with ioMRI and based in our experience a rationale has been performed to better understand the usefulness of this tool and to provide surgical guidelines for the future in pediatric neurosurgery.

\section{OP18}

Role of ultrasonographic evaluation in suspected craniosynostosis

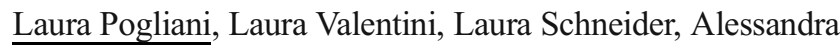
Erbetta, Elena Beretta, Valentina Giudici, Gianvincenzo Zuccotti (Milan, Italy)

Prevalence of craniosynostosis (CS) is estimated at 1 in 2500 live births. Differential diagnosis might be difficult as misdiagnosed with positional plagiocephaly, which dramatically increased since adoption of AAP guidelines to prevent sudden infant death syndrome. Clinical presentation allows a reliable diagnosis in most cases, but when specific features are missing further examination becomes mandatory. The gold standard for the diagnosis is computed tomography (CT) but it involves sedation, radiation exposure and is expensive. Ultrasound scan (US) is a promising tool as a first step investigation.To assess US diagnostic accuracy, we evaluated 137 infants (92 males) aged from 3 days to 12 months (mean age 4.3 months), clinically suspected to have CS with US scan (LogiQ 5, General electric, 7.5MHZ linear probe) and, if a suspicion of stenosis was raised, infants were referred to 3DCT scan, otherwise a routine clinical follow-up was carried out. US was inconclusive in 4 cases due to hypermotility of the infants (normal CT scan). In 18/18 infants the diagnosis was confirmed by CT ( 9 cases of sagittal, 4 metopic, 2 coronal, 1 partial coronal, 1 lamdoyd, 1 sagittal plus metopic stenosis), while 11 infants underwent CT because of non reassuring clinical presentation, and result was normal. None of the 108 infants who underwent the clinical follow up was diagnosed as to have a CS later. Our data show excellent correlation between US and $\mathrm{CT}$ in diagnosis of CS. US scan is an effective, fast, low cost, easy to perform, radiation-free procedure and should become the screening method for children with head deformity while CT may be reserved for those with abnormal US and preoperative planning.

\section{OP19}

Transorbital ultrasound measurement of the optic nerve sheath diameter (ONSD) as a non-invasive marker of intracranial pressure (ICP) in children

Llewellyn Padayachy, Ushma Galal, Anthony Figaji, Fieggen A. Graham (Cape Town, South Africa)

Purpose: To analyze the diagnostic accuracy of age-related optic nerve sheath diameter (ONSD) cut-off values in children for detecting raised intracranial pressure (ICP). 
Methods: ONSD measurement was performed prior to invasive ICP in children under general anesthesia, undergoing a neurosurgical procedure. The diagnostic accuracy of the ONSD measurement was compared to ICP at thresholds of $20,15,10$ and $5 \mathrm{mmHg}$. This was further analyzed in children above and below the age of 1 year, with a subgroup analysis of age at 4 years old in part II of this study.

Results: Data from 174 children were analyzed. In children $\leq 1$ year old the ONSD measurement with the best diagnostic accuracy for detecting ICP $\geq 20 \mathrm{mmHg}$ was $5.16 \mathrm{~mm}$, compared to $5.75 \mathrm{~mm}$ in children $>1$ year old $(\mathrm{p}<0.001)$. In addition, patency of the anterior fontanelle (AF) was found to be a useful clinical marker for defining different ONSD cut-off values at ICP thresholds of 20, 15, 10 and $5 \mathrm{mmHg}$.

Conclusion: Transorbital ultrasound measurement of the ONSD is a reliable non-invasive marker of ICP particularly at higher thresholds of 20 and $15 \mathrm{mmHg}$, in children. Patency of the AF is a useful clinical marker for defining different ONSD cut-off values in children.

\section{OP20}

Validity evidence for the neuro-endoscopic ventriculostomy assessment tool (NEVAT)

Gerben Breimer, Faizal Haji, Giuseppe Cinalli, Eelco Hoving, James Drake (Amsterdam, the Netherlands; London ON, Canada; Naples, Italy; Groningen, the Netherlands; Toronto ON, Canada)

Background: Growing demand for transparent and standardized methods for evaluating surgical competence prompted the construction of the Neuro-Endoscopic Ventriculostomy Assessment Tool (NEVAT).

Objective: To provide validity evidence of the NEVAT by reporting on the tool's internal structure and its relationship with surgical expertise during simulation-based training.

Methods: The NEVAT was used to assess performance of trainees and faculty at an international neuroendoscopy workshop. All participants performed an endoscopic third ventriculostomy (ETV) on a synthetic simulator. Participants were simultaneously scored by 2 raters using the NEVAT procedural checklist and global rating scale (GRS). Evidence of internal structure was collected by calculating interrater reliability and internal consistency of raters' scores. Evidence of relationships with other variables was collected by comparing the ETV performance of experts, experienced trainees, and novices using Jonckheere's test (evidence of construct validity).

Results: Thirteen experts, 11 experienced trainees, and 10 novices participated. The interrater reliability by the intraclass correlation coefficient for the checklist and GRS was 0.82 and 0.94, respectively. Internal consistency (Cronbach's a) for the checklist and the GRS was 0.74 and 0.97 , respectively. Median scores with interquartile range on the checklist and
GRS for novices, experienced trainees, and experts were 0.69 (0.58-0.86), 0.85 (0.63-0.89), and 0.85 (0.81-0.91) and 3.1 (2.5-3.8), $3.7(2.2-4.3)$ and $4.6(4.4-4.9)$, respectively. Jonckheere's test showed that the median checklist and GRS score increased with performer expertise $(\mathrm{P}=.04$ and .002 , respectively).

Conclusion: This study provides validity evidence for the NEVAT to support its use as a standardized method of evaluating neuroendoscopic competence during simulation-based training.

\section{OP21}

Diagnostic yield and clinical impact of brain biopsy in children presenting with neurological deterioration of unknown cause

Jane Hassell, Patrick Grover, Charlotte Sayer, Cheryl Hemingway, Kristian Aquilina (London, United Kingdom)

Introduction: The role of brain biopsy in neurological deterioration of uncertain cause is controversial. The yield and value of diagnosis must be weighed against the risk of potentially catastrophic complications. We retrospectively reviewed indications for and outcomes of biopsy over a 25 -year period at a UK quaternary Paediatric centre.

Methods: Patients were identified using computerised codes for the period January 1990 to December 2015. All children (0-18 years) who presented with neurological deterioration and underwent biopsy after negative or inconclusive noninvasive diagnostics were included. Cases with a clear radiological diagnosis of tumour were excluded. Case notes, MRI, histopathology, microbiology and virology results were reviewed. We identified cases where histopathology had influenced further management.

Results: Fifty-three brain biopsies were performed over 25 years, 27/53 (50.9\%) in 2009-15. Complete data were available for $43 / 53$ (81.1\%). The main indications for biopsy were a differential diagnosis including malignancy, or to exclude contraindications to immunosuppressive treatment. Brain histology was abnormal in 42/43 (97.7\%) cases. Abnormal findings included unspecified encephalitis (28.6\%); diffuse neoplasm (23.8\%); specific infection (7.1\%); granulomatous disease (7.1\%); Rasmussen encephalitis (7.1 and non-specific changes (23.8\%). Biopsy was diagnostic in 31/43 (72.1\%). Biopsy findings altered clinical management in $36 / 43$ $(83.7 \%)$ cases. Short-term complications occurred in $6 / 43$ (14\%). There were no long-term complications.

Conclusions: Brain biopsy was safe and effective with a diagnostic yield of $72.1 \%$, clinical impact on management in $83.7 \%$ cases and short-term complication rate of $14 \%$. Our diagnostic yield from brain biopsy in children was higher than that reported from biopsy for adult-onset cryptogenic neurological disease (1). Clinicians and parents are often reluctant to undergo biopsy and this review may support decision-making. 
Reference: Bai et al., Diagnostic Value and Safety of Biopsy in Patients with Cryptogenic Neurological Disease: A Systematic Review and Meta-analysis of 831 Cases. Neurosurgery 2015; 77: 283-295.

\section{OP22}

A pilot study of the effects of selective dorsal rhizotomy in children with severe spastic cerebral palsy, GMFCS IV, at one year

Kristian Aquilina, Deepti Chugh, Stephanie Cawker, Jacqueline Gordon, Neil Wimalasundera, Lucinda Carr (London, United Kingdom)

Introduction: The short and long-term benefits of selective dorsal rhizotomy (SDR) for children with spastic cerebral palsy (CP), GMFCS II and III, have been described. We questioned whether children with more severe CP (GMFCS level IV) may also benefit from SDR.

Methods: Children with bilateral, predominantly spastic CP, GMFCS level IV, selected for SDR in a multidisciplinary clinic were evaluated in this prospective cohort study. Objective goals were agreed with parents. Baseline and outcome data were collected over 6-18 months postoperatively. Changes in spasticity, motor function and quality of life were tracked using Ashworth scores, GMFM -88 and 66 (Gross Motor Function Measure), and CP Qual.

Results: 10 children, 9 male, median age 5.3 years at surgery (range 3.5- 9.8), underwent SDR between 2013 and 2014. Spastic tone was abolished (Ashworth 0) in all. Muscle tightness with dystonia (mixed tonal pattern) was evident in most before, and persisted after SDR. GMFM-88 (66) improved from baseline median of 44.7 to 57.3 (42.5 to 45.8$)$ at $6-18$ months post-SDR. Most improvement occurred in the B (sitting) and $\mathrm{C}$ (crawling and kneeling) domains (63.3 to 81.7 and 52.4 to 66 ) respectively. All parents reported positive effects from SDR, evident on CP Qual and PEDI scores.

Conclusion: SDR significantly reduces spasticity in children with CP GMFCS level IV. Improvements were most marked in floor mobility and posture. Dystonia is not improved with SDR. None of the children deteriorated. The GMFM- 88 scale is more sensitive than the GMFM-66 to post-operative changes. With clear goal setting, SDR should be considered as a potentially useful intervention in these children.

\section{OP23}

\section{Developing a national pediatric neurosurgical network in} the Netherlands

Erik J. van Lindert ${ }^{1}$, Martine van Bilsen ${ }^{1}$, Marie-Lise van Veelen $^{2}$, the Dutch Pediatric Neurosurgery Society (Nijmegen, the Netherlands; Rotterdam, the Netherlands)

Introduction: Pediatric neurosurgery is performed in The Netherlands in the 8 University Hospitals of the country.
Each centre has 1-3 pediatric neurosurgeons ( $\mathrm{PN})$. There is no centre that is able to cover all aspects of pediatric neurosurgery and therefore numerous subspecializations exist among the different centers. This creates an urge for further centralization of care. However, such a physical centralization comes along with serious problems and objections as well. The Canadian Pediatric Neurosurgery Study Group (CPNSG) has set an example of collaboration in research that the Dutch PN wanted to follow: establishing a Dutch Pediatric Neurosurgery Study Group (DPNSG). However, we intend to take this one step further and collaborate not only for scientific questions, but also for routine pediatric neurosurgical care, education, training, patient information and patient communication, and thus establish a virtual pediatric neurosurgery centre in The Netherlands. Because it is intended to improve pediatric neurosurgical care in the broadest sense, we also seek collaboration with the patient and his caretakers.

Methods: A platform for collaboration is created, the Dutch Pediatric Neurosurgery Network (DPNN), in which all PN of The Netherlands collaborate. Patients join in through a patient advisory board. The platform consists of a website with a secured digital environment.

Results: The digital platform offers: 1) information on pediatric neurosurgical disease and treatment; 2) an online community for patients; 3 ) an online outpatient clinic in part replacing physical contact (Facetalk); 4) an online secured environment for pediatric neurosurgeons for collaboration, mutual consultations, conferences, protocols and standards as well as collaborative research. The outline for this platform has been set, the first grant has been received and the platform is now in the process of development.

Conclusion: A national pediatric neurosurgery network encompassing all aspects of pediatric neurosurgery is a unique collaborative initiative made possible by modern technology and will bring pediatric neurosurgery to a higher level.

\section{OP24}

Children's Epilepsy Surgery Service in England: preliminary audit of activity and outcome

William Harkness ${ }^{*}$, J. Helen Cross ${ }^{*}$, Sophia Varadkar, Shakti Agrawal, Sunny Philip, Richard Walsh, Tim Martland, Michael Carter, Christopher Verity ${ }^{*}$ (on behalf of National CESS Team NHS England) (London, United Kingdom; Birmingham, United Kingdom; Manchester, United Kingdom; Liverpool, United Kingdom; Bristol, United Kingdom; Cambridge, United Kingdom)

* Advisors to NHS England for CESS

Objective: To examine trends in epilepsy-related surgical procedures (ERSP) in children performed at the four Children's Epilepsy Surgery Service (CESS) centres in England since inception of the service in November 2012, to analyse 
outcome and follow-up data at one year, to identify potential treatment gaps in different geographical areas and to reflect on challenges encountered and on-going, since the inception of the CESS.

Methods: Epilepsy-related surgical activity was compared for the first 20 months of CESS service (Nov 2012-June 2014 first period) with the more recent period (April 2014-Sep 2015 - second period). Data were collected from each of the four CESS centres with regard to referrals, case evaluation, surgical procedures and outcome. The number of referrals to CESS for the commissioning year 2014-15 was compared to the expected number based on an epidemiological cohort. ${ }^{1}$ Adverse surgical outcomes were measured using the GOSH adverse surgical grading score.

Results: There were 183 ERSP for the first period, compared to 271 in the second period representing time-adjusted increase in activity of $61 \%$. In 2014-15 commissioning year, 387 children were referred for consideration of epilepsy surgery to the 4 CESS centres, representing between $30-50 \%$ of expected numbers based on the previous epidemiological cohort. There is a very considerable variation in referral rates within the different regions of the UK. Twelve months following surgery, 95 (58\%) of children were Engel Class I (seizurefree) with 141 (87\%) Engel Class I-III one year following surgery. Best outcomes were achieved with temporal lobe surgery with $77 \%$ seizure-free at 1 year. There were no mortalities but two unexpected surgery-related morbidities in this group.

Conclusion: We have seen a major change in the children's epilepsy surgery landscape in England since the inception of CESS. There has been a significant yearby-year increase in ERSP during the study period. Outcomes by Engel class remain equivalent to published international outcomes.

\section{Session 5: Oncology}

\section{OP25}

Childhood craniopharyngeoma: 15 years of multimodal treatment experience

Patricia Dastoli, Sergio Cavalheiro, Amanda Lopez, Valérie Manicacci, David Bevilacqua, João Gomes, Mariana Lima, Jardel Nicácio, Andrea Cappellano, Nasjla Silva (Sao Paolo, Brazil)

Introduction: Treatment strategies for patients with craniopharyngioma is still controversial. Gross total resection was advocated for many years, regarding post operatory clinical conditions. Currently, quality of life is the goal for all treatment modalities.

Objective: To analyze the several treatment strategies and outcomes of craniopharyngioma patients in 15 years of experience in a single institution.
Methods: The authors retrospectively evaluated 101 craniopharyngeoma patients treated between 2000 and 2015, at IOP/GRAACC/UNIFESP. Data regarding initial clinical presentation, body mass index (BMI), endocrinological complications, treatment modalities and outcomes were collected. Results: From 101 patients treated with craniopharyngioma at our institution, 78 patients were elegible for evaluation and 13 excluded for loss of follow-up. Forty- four were boys and 34 girls. The mean age at treatment was 10.5 years (range 3.2525.08). Fifty-six percent of children presented with signs and symptoms of intracranial hypertension, $24 \%$ with visual impairment and $38.5 \%$ with endocrinological disturbances. At the time of diagnosis, the mean BMI was $20.48 \mathrm{~kg} / \mathrm{m}^{2}$ (range 12.7- 32.8). Predominantly cystic tumors (cystic portion $\geq 60 \%$ of total lesion volume) occurred in 31 children and were treated with intralesional interferon-alpha; of these patients, $48 \%$ (15/31) progressed to surgical intervention and adjuvant radiotherapy. Among the 47 predominantly solid tumors, 3 were treated with microsurgery only, 32 with microsurgery + radiotherapy and 12 with endoscopic transesphenoidal surgery + radiotherapy. The mean BMI of patients treated exclusively with interferon-alpha was $22.88 \mathrm{~kg} / \mathrm{m}^{2}$ (ideal weight), with interferon-alpha + microsurgery was $31.23 \mathrm{~kg} / \mathrm{m}^{2}$ (grade 1 obesity) and microsurgery + radiotherapy was $29.65 \mathrm{~kg} / \mathrm{m}^{2}$ (overweight). After treatment, 51 cases (65\%) progressed to panhypopituitarism. The mean follow up was 7.13 years (range 1-17), and 8 deaths occurred. Currently 34 patients are out of treatment.

Conclusion: Intracystic therapy with interferon-alpha offers best body mass index control. However, panhipopituitarism is the most common and important complication irrespective of treatment modalities.

\section{OP26}

Benefits and risks of neoadjuvant chemotherapy in metastatic medulloblastoma: a comparative study in 71 patients

Lea Guerrini-Rousseau, Christelle Dufour, Stephanie Puget, Virginie Kieffer-Renaux, Marie-Anne Raquin, Pascale Varlet, Audrey Longaud, Christian Sainte-Rose, Dominique ValteauCouanet, Jacques Grill (Villejuif, France)

Background: Preoperative chemotherapy is often used in pediatric oncology to treat metastatic disease and facilitate the surgery of the primary tumor, but not for brain tumors. A first pilot study showed in 2004 the feasibility and effectiveness of preoperative chemotherapy in treating high-risk medulloblastomas.

Methods: Seventy-one patients were treated for a metastatic medulloblastoma between 2002 and 2010 at Gustave Roussy. Two strategies were compared in intention to treat. Forty-two children were operated at the time of the diagnosis (group A) and the 29 others children were treated with 2 courses of 
carboplatin and etoposide (group B) after histological diagnosis (biopsy) and before a delayed surgery. Children of group A received the two courses of carboplatin and etoposide afterwards. The rest of the protocol (high-dose chemotherapy and cranio-spinal irradiation) was similar in the both groups. Complete neuropsychological testing, including intelligence quotient measurements, was scheduled before surgery, before radiotherapy and every year thereafter.

Results: MRI performed after neo-adjuvant chemotherapy showed an objective response in 24 patients (83\%) and a stable disease in 4 patients (14\%). Complete excision rate was significantly higher in group B $(100 \%$ versus $64 \%$, $\mathrm{p}=0.00248$ ) without any difference in postoperative complication rate. Medulloblastoma cells could still be evidenced despite preoperative chemotherapy. The median Total Intelligence Quotient (TIQ) was 81 and 90 in group A and B respectively $(p=0.02543)$. This difference was even more significant in young children (77 vs 91 points for groupe A and B, respectively). Hydrocephalus and brain radiotherapy dose were not associated with TIQ. Neo-adjuvant chemotherapy had no negative impact on local disease control ( 79 versus $83 \%$ ). Event-free survival and overall survival at 3 years were 54 versus $62 \%$ and 58 versus $68 \%$ respectively $(\mathrm{p}=\mathrm{NS})$.

Conclusion: Neo-adjuvant chemotherapy in metastatic medulloblastoma is safe and could have a positive impact on neuropsychological outcome and completeness of surgery.

\section{OP27}

Long-term follow-up following surgical treatment of intramedullary spinal cord pilocytic astrocytomas in children

Francesco Sala, Ema Tot, Franco Faccioli, Albino Bricolo, Carlo Mazza (Verona, Italy)

Introduction: The surgical treatment of intramedullary spinal cord astrocytomas (ISCA) in children is still controversial. We retrospectively reviewed our experience with pilocytic ISCA in 24 patients in order to identify the role of clinical and treatment-related factors in determining survival and neurological outcome.

Material and Methods: We reviewed clinical charts, neuroimaging studies and surgical reports of all children (age $\leq 16$ years) operated on for pilocytic ISCA between 1983 and 2013. Neurologic function was graded according to the McCormick scale. Follow-up data of all contactable patients were updated through phone interviews. Since 2000, all surgical procedures were performed under neurophysiological surveillance with somatosensory and motor evoked potential monitoring.

Results: Mean clinical history was 14 months. On admission most of the patients were on McCormick grade II $(n=13)$ or I $(\mathrm{n}=6)$. Surgical removal was gross total $(>95 \%)$ in 10 cases, subtotal $(>75 \%)$ in 13 , and partial in one case. One child received adjuvant therapy.

At a mean radiological follow-up of 43 months, three children were lost to follow-up while the other 21 were all alive with either no disease $(\mathrm{n}=7)$, stable residual tumor $(\mathrm{n}=11)$, or slow progression $(n=3)$. At the last clinical follow-up of 98 months, $10 \%$ of the children worsened, $32 \%$ improved, $58 \%$ were unchanged, as compared to pre-operatively.

Conclusion: Surgery is the treatment of choice for pilocyitic ISCAs, and it should be performed precociously as the major determinant of long term neurological outcome is the preoperative neurological status. Even subtotal resection warrants long-term survival with good neurological outcome in over $90 \%$ of the cases.

\section{OP28}

Surgery of subependymal giant cell astrocytomas in tuberous sclerosis complex. Surgical outcome and morbidity in 31 patients and 44 surgical procedures

Flavio Giordano, Barbara Spacca, Elena Arcovio, Pierarturo Donati, Manuela Grandoni, Regina Mura, Federico Mussa, Giacomo Peri, Massimiliano Sanzo, Mirko Scagnet, Lorenzo Genitori (Florence, Italy)

Introduction: Subependymal Giant Astrocytomas (SEGAs) are intraventricular tumors commonly associated with Tuberous Sclerosis Complex (TSC). Surgery is the recommended treatment though morbidity is not negligible. We report our surgical experience upon a series of 44 surgical procedures in 31 patients.

Methods: Since 1994 to 2015, 38 patients with SEGA out of 89 with TSC (42.7\%) came to our observation: 19 males, 19 females; mean age 11.3 years (6 months-34.3 years). SEGAs were mostly located into caudate nucleus (33); 7 patients harbored multiple SEGAs. Pre-operative hydrocephalus occurred in 14 cases $(36.8 \%)$ ). 31 patients received surgery because of tumor growth (22), oncological issues (5), and raised intracranial pressure (4). Multiple SEGAs were preferantially removed in staged procedures.

Results: 44 surgical procedures were performed: 38 Gross Total Removal (GTR), 6 Subtotal Removal (STR). Surgical approaches were transcallosal (25), transventricular (16) and neuroendoscopic (3). 13 patients underwent multiple surgeries for multiple SEGAs and/or recurrences. Surgical morbidity accounted for $22.7 \%$ (10/44): subdural hygroma/hematoma (3), acute hydrocephalus (2), wound infection (1), memory deficit (1), hemiparesis (1), cerebral edema (1), and fatal myocardial infarction (1) leading to $2.3 \%$ operative mortality. Mean follow-up was 59.4 months (1 month-17.6 years). Tumor recurrences occurred in $11 / 30$ patients $(36.7 \%)$ after a mean time of 29.7 months ( $2-75$ months), 6 of them after STR: 9/11 received GTR, 2 Everolimus treatment. At last control $27 / 30$ patients $(90 \%)$ showed the same neurological status as 
before surgery, 3 worsened (10\%); 28/30 (93.3\%) had no evidence of SEGA disease; $12 / 30$ had a VP-shunt (40\%).

Conclusion: Despite the significant morbidity (22.7\%), radical surgery of SEGAs confirms its efficacy in tumor control since at last follow-up 93.3\% (28/30) patients had no evidence of disease. Furthermore, the neurological status was not deteriorated in $90 \%(28 / 30)$ patients after surgery.

\section{OP29}

Intracranial neoplasms in the first year of life: results of a third cohort of patients from a single institution

Sebastian M. Toescu, Gregory James, Kim P. Phipps, Owase Jeelani, Dominic Thompson, Kristian Aquilina (London, United Kingdom)

Introduction: Brain tumors in the first year of life are rare and their management is challenging. The objective of this large cohort study is to characterize the contemporary management of brain tumors in infants with reference to previous institutional series (Kane et al., 1999, Jooma et al., 1984).

Methods: Electronic/paper case note review of all brain tumors diagnosed at our institution in children aged $<1 \mathrm{yr}$ since the publication of our previous series, i.e. from April 1997 to January 2014.

Results: 100 patients were seen; 56 were male. Median age at presentation was $187 \mathrm{~d}$ (1-354); mean duration of symptoms was $50 \mathrm{~d}$. The most common presentations were vomiting and macrocrania. $63 \%$ of tumors were supratentorial, $37 \%$ infratentorial. 92 patients underwent 224 procedures; 8 patients had no surgery. 119 operations were directly on brain tumours (biopsy 39, debulking 47, gross total resection 34). 89 CSF diversions, 10 endoscopic procedures, and 13 pre-operative embolizations were performed. Operative mortality was $2 \%$. The commonest tumors were choroid plexus neoplasms (17 papillomas (CPP), 5 carcinomas) $>$ PNET (12) >ATRT (10) > GBM (9) >optic glioma (9) $>$ ependymoma (8) > LGG (6) > pilocytic astrocytoma (6) >teratoma (5), with 13 miscellaneous tumors. Survival was $93 \%$ at $1 \mathrm{~m}(93 / 100), 65 \%$ at $1 \mathrm{y}(62 /$ $96), 43 \%$ at $5 y(32 / 74), 27 \%$ at $10 y(15 / 56)$. No patients with CPP or LGG died. 5-year survival rates were lowest for anaplastic ependymoma/PNET/teratoma $(0 \%)$ and ATRT (11\%). 53\% of children reaching school age were in mainstream school, $30 \%$ assisted and $17 \%$ special school.

Conclusions: Overall survival is unchanged compared to previous series. However, there was an increase in the number of CPP and ATRT diagnosed, illustrating the two extremes of prognosis in neonatal brain tumors. Future directions for diagnosis and management of this complex group of infants will be considered.

\section{OP30}

Robot-assisted stereotactic biopsy in DIPG

Andrea Carai, Alessandro De Benedictis, Raffaella Messina, Giacomo Esposito, Franco Randi, Alessandra Savioli, Angela Mastronuzzi, Carlo Efisio Marras (Rome, Italy; Brescia, Italy)

Introduction: Recent advancements in molecular characterization of diffuse intrinsic pontine gliomas (DIPG) have favored the reintroduction of stereotactic biopsy to obtain tumor samples. Acceptable morbidity has been documented in experienced Centers performing biopsies in clinical trials. Transcerebellar transpeduncular approach has been proposed as a safer and easier way to perform pontine biopsies with favorable risk profile (5-10\% of new neurological deficit).

Methods: Moving from the vast experience of the senior author in stereotactic and functional neurosurgical procedures we report our series of robot-assisted stereotactic biopsies for DIPG in children. From January 2015 to January 2016 six biopsies were performed using the Rosa robotic arm system using a transfrontal precoronary approach. Whenever feasible target was chosen in high diffusion restriction areas or contrast enhancing areas on MRI imaging and fused with CT scan images to maximize precision.

Results: Four to six samples were obtained from each procedure allowing histological characterization and H3K27 mutation identification in all cases. We were able to save freshly frozen samples for biological characterization to guide target therapy in all cases.

We observed transient worsening of diplopia in two patients, returning to baseline within 1 month. No bleeding was documented at early CT scan studies along the needle track. The target, documented by presence of subtle air bubbles, was reached as preoperatively planned in all cases.

Conclusion: Robot assisted stereotactic biopsy has demonstrated an acceptable risk profile in experienced hands. Transfrontal approach has the theoretical advantage to be parallel to white matter long tracts of the pons, however a longer track through brain structures is required. Further advantages are supine position during the procedure and possibility to used robotic systems with submillimetric precision and high stability of biopsy needle. Whatever the technique this procedures should better be performed at selected Centers offering clinical trials for DIPG.

\section{OP31}

Brain tumors associated with early epilepsy onset: approach and outcome

Helio Machado, Marcelo Volpon-Santos, Ricardo SantosOliveira, Francisco Scornavacca, Ursula Thome, Marilia Bernardino, Ana Hamad (Ribeirao Preto, Brazil)

Brain tumors represent the second most frequent etiology of surgical cases in children. We discuss herein a consecutive 
series of patients who were submitted to epilepsy surgery from 1996 to 2013 at a dedicated center emphasizing specific aspects of such cases.

Patients underwent clinical, neuropsychological, electroencephalographic and video-EEG investigations as part of a protocol for refractory epilepsy that included imaging studies in order to outline an epileptogenic zone. Subsequently, a surgical decision was made either by lesionectomy or extended surgery, with the aid of electrocorticography (ECoG) or prolonged invasive monitoring.

We identified 56 cases treated during that period. In average, seizures started at the age of 6 years and soon became refractory to anti-epileptic drugs (AED). Mean age at diagnosis was 9.7 and 11.3 at the time of surgery. Seizures were complex partial, daily ( $50 \%$ of cases) and video-EEG showed focal alterations ( $85 \%$ of cases). ECoG was used in $37 \%$ of cases followed by chronic invasive monitoring in $15 \%$ of patients. Temporal lobe was the most frequent location (59.3\%) with eloquent areas involved in $18.5 \%$ of cases. Lesionectomy was performed in $66.7 \%$ of cases and in $33.3 \%$ extended surgery was necessary. Histologically, DNET and Gangliogliomas represented $33.3 \%$ of cases each. In $26 \%$ of cases, type IIIb cortical dysplasia was present. At 5 years of follow up $90 \%$ were seizure free, and cognition improved in $31.3 \%$ of patients.

Brain tumors represent a frequent etiology of epilepsy in patients undergoing surgical treatment in the pediatric age group. For these patients, a different approach is imperative, in a multidisciplinary epilepsy center. The aim is to detect the epileptogenic zone which is frequently larger than the lesion itself. Results of treatment are favorable concerning both seizure control and cognition.

\section{OP32}

\section{Pediatric transsphenoidal surgery of sellar localization} tumors

Pavlo Plavskyi, Mykola Guk, Oksana Skobska, Arthur Mumlev (Kiev, Ukraine)

Introduction: Transsphenoidal approach is limited for the use in paediatric patients of early age. Contemporary achievements of neuroendoscopic technique significantly increase possibilities of transsphenoidal surgery in children.

Methods: During 2011-2015 at the Neurosurgery Institute 26 transsphenoidal surgeries were performed in 24 pediatric patients. There were 12 craniopharyngiomas ( 2 recurrent), 8 pituitary adenomas, 2 -glyomas, 1 -Rathke cyst, 1 -germinoma of 3 rd ventricle. The youngest patient was 3.6 years old (cystic craniopharyngioma), the oldest patient was 17.5 years old (prolactinoma). In surgical planning the complex assessment of the rhinoscopy, MRI and CT of paranasal sinuses data were used. In patients of early age the 3D modelling (3D-Slicer software) was used. We performed the binostral endoscopy (if possible) or microsurgical transseptal transnasal approach with some modifications. During the removal stage $0^{\circ}, 30^{\circ}$ and $45^{\circ}$ endoscopes were used. In 2 patients also the exoscope Vitom was used, in 4 patients the neuro-navigation was used, in 9 patients the intrasurgical fluoroscopy was used.

Results: There were no intrasoperative complications. In all patients the goal of a surgery was achieved (verification of a diagnosis, total or subtotal removal of a tumour with the decompression of an optic chiasm). In the age group of 12-18 years old patients there were almost no differences from surgeries in adult patients because in all patients the distance between ICAs was more than $12 \mathrm{~mm}$, and the pneumatisation of a sphenoid sinus was similar to an "adult anatomy"; the surgical access was problematic in patients younger than 6 years old due to the subtotal sphenoid sinus formation and very narrow nasal passages. Thus, if the nostril diameter is less than 7-8 $\mathrm{mm}$, the binostral endoscopy cannot be performed. We preferred the transseptal approach with the transverse section of a cutaneous part of a nasal septum with the special nasal retractor use (the retractor was selected based on pre-surgery planning data).

Conclusion: In the presence of the necessary technical equipment and surgeons' experience, the transsphenoidal approach in children can be deemed safe and adequate for some sellar tumours. For suprasellar lesions the promising direction is the use of extended transsphenoidal endoscopy, but its performance is limited by anatomical features of children of early age groups.

\section{OP33}

Ultrasound-induced opening of the blood-brain barrier to enhance temozolomide and irinotecan delivery to the brain:an experimental study in rabbits

Kévin Beccaria, Michael Canney, Lauriane Goldwirt, Christine Fernandez, Julie Piquet, Marie-Cécile Perier, Stéphanie Puget, Cyril Lafon, Jean-Yves Chapelon, Alexandre Carpentier (Paris, France; Châtenay-Malabry, France; Lyon, France)

Object: The blood-brain barrier (BBB), limits the intracerebral penetration of drugs and brain tumor efficacy. It has been shown that ultrasound (US) may transiently and safely open the BBB. In the present study, we assessed the effect of ultrasound-induced opening of the $\mathrm{BBB}$ on the intracerebral concentration of temozolomide (TMZ) and irinotecan (CPT-11).

Methods: The experiments were performed using 34 healthy New Zealand rabbits. Half had a unilateral BBB opening after craniectomy, and half served as controls with a unilateral craniectomy without sonication. Sonications were performed by pulsing a $1.05-\mathrm{MHz}$ planar ultrasound transducer with a duty cycle of $2.5 \%$ and an in situ acoustic pressure level of $0.6 \mathrm{MPa}$ after injection of a microbubble ultrasound contrast 
agent $\left(\right.$ Sonovue $\left.^{\circledR}\right)$. Drugs were injected either 5 minutes before (ChemoPreUS) or 15 minutes after (ChemoPostUS) the ultrasound sonication. The plasma and intracerebral concentrations of both drugs were quantified using ultra-performance liquid chromatography.

Results: The mean intracerebral tissue-to-plasma drug concentration ratio in the control hemispheres was $34 \%$ for TMZ and 2\% for CPT- 11 . After BBB opening, these values increased by up to $21 \%$ for TMZ and up to $178 \%$ for CPT- 11 . Intracerebral concentrations of drugs were enhanced in regions where the $\mathrm{BBB}$ was opened compared with the contralateral hemisphere $(p<0.01$ and $p<0.0001$ for CPT-11, $\mathrm{p}=0.02$ and $\mathrm{p}=0.03$ for TMZ, in ChemoPreUS and ChemoPostUS, respectively) and compared with the control group ( $\mathrm{p}<0.001$ and $\mathrm{p}<0.0001$ for CPT-11, $\mathrm{p}<0.01$ and $\mathrm{p}=0.02$ for TMZ, in ChemoPreUS and ChemoPostUS, respectively). The intracerebral distribution of drugs was heterogeneous, depending on the distance from the ultrasound source and sonicated tissue (grey matter versus white matter). Conclusions: Ultrasound-induced opening of the BBB significantly enhances the intracerebral concentration of both TMZ and CPT-11 in rabbits.

Funding: CARTHERA and the Paris Regional Innovation Center supported this work.

\section{OP34}

Atypical teratoid/rhabdoid tumors - A 10 year single institution experience

Thomas Jaques, Kirstian Aquilina, Dolin Bhagawati, Kirn Sandhu, Kim Phipps, Samantha Hettige, Magda Garzon, Owase Jeelani, Dominic Thompson (London, United Kingdom)

Introduction: Atypical Teratoid/Rhabdoid Tumours (ATRT) are rare, highly malignant tumours of the central nervous system with a dismal prognosis under the age of 3 . We present a large single institution series and discuss surgical management and prognostic factors.

Methods: We performed a retrospective analysis of case notes and imaging for children with a histological diagnosis of ATRT treated over a 10-year period in a tertiary paediatric neurosurgery unit. Data on demographics, tumour volumes, genetics, treatment and survival were collected. All patients were managed via the local neuro-oncology multidisciplinary group.

Results: 25 patients (M:F=11:14), presenting between 2005 and $2015 ; 19(76 \%)$ where treated in the last 5 yearsMean age at diagnosis was 2.5 years. $18(72 \%)$ were infratentorial and 7 $(28 \%)$ were supratentorial. $8(32 \%)$ required primary CSF diversion, and a further $3(12 \%)$ required a CSF diversion procedure after primary surgery. $8(32 \%)$ were treated palliatively (no oncological therapy), of whom 5 were under the age of 1 . Overall mean survival was 1.6 , with mean survival 1.0 under the age of 1 and 1.9 years over the age of 1 . For those receiving treatment, mean survival was 2.16 years. Extent of tumour resection significantly affected overall survival (mean $83.23 \%$, median $95.99 \%, \mathrm{p}<0.001$ ). Within the fully treated group, this was also evident (mean resection $97.114 \%$, median $100 \%, \mathrm{p}<0.001)$.

Conclusions: ATRT remains a tumour with extremely poor prognosis; an aggressive surgical strategy confers a significant survival benefit.

\section{OP35}

Role of intraoperative MRI in resection of pediatric anterior skull base tumor - Initial experience

Santosh Mohan Rao Kanangi, Sonia Tejada, Shivram Avula, David Richardson, Ajay Sinha (Liverpool, United Kingdom)

Introduction: Skull base tumours in paediatric neurosurgery are infrequent and are a tricky proposition. The nature of the tumours ranges from being indolent and benign to highly malignant and invasive. The use of the intra-operative MRI (ioMRI) guided tumour resection allows us to achieve a higher degree of surgical clearance with minimal damage.

Materials and Methods: This is a retrospective data analyses of cases operated at Alder Hey between 2010-2015. The data has been analyzed for 15 procedures $(n=15)$. The age range was from 8-16 years. The location of the lesions were as follows: Pituitary tumours (5), Sellar-suprasellar tumours (6), orbitofrontoethmoid (1), anterior skull base (1), orbitoethmoidal (1), clival (1). The type of procedure, pathology and the outcome have been analyzed. The approaches used were endoscopic transnasal trans-sphenoidal (11), Combined approaches (orbital and frontal) (1), LeFort 1 procedure in 2, bifrontal craniotomy in 1 . The intent, prior to surgery (whether total/radical/subtotal excision or biopsy) was compared with the result after intial resection i.e. after the intra-op MRI and at the completion MRI study. The tumour volumes prior to and at the end of the procedure were also measured.

Results: Intra-op MRI aided in the complete excision of the lesion in 7 cases, radical debulking in 6 cases and subtotal excision in 2 cases. There were no MRI safety issues. The discrepancy between the surgical impression of the extent of resection and the intra-operative MRI findings has been recorded. Since most of these lesions are close to the bone, to clearly ascertain the quanta of residual tumour on the scan is challenging.

Data gathered showed that though ioMRI has its technical limitations it still is very useful in aiding a more complete tumour resection. IoMRI resulted in the patient being taken in for a second procedure for residual tumour prior to closure in 4 cases. The histology of the lesions were myriad and were as follows: Epitheloid sarcoma (1), pituitary adenomas (6), 
chordoma (1), craniopharyngioma (4), Juvenile nasopharyngeal angiofibroma (JNA) (1), atypical meningiomas (2).

Complications included CSF leak in 2, new endocrine disturbances in 1, diabetes insipidus in 1, vasospasm and limb weakness in 1 . Recurrences occurred in 3 patients (all pituitary adenomas) and warranted re-surgery. The data along with existing literature on this topic will be discussed.

Conclusion: Intra-operative MRI is a very valuable adjunct in the resection of paediatric skull base lesions. However, we have limited experience at this point in time.

\section{OP36}

How to increase the percentage of complete resections for ependymoma? Results of a survey

Marie-Lise Van Veelen, Guirish Solanki (Rotterdam, the Netherlands; Birmingham, United Kingdom)

Complete resection for ependymoma can increase survival by around $30 \%$, but at the expense of a comparable increase in percentage of (post) operative complications. Percentage of complete resections vary widely between studies, ranging from $50 \%$ to $80 \%$.

The SIOP Ependymoma II protocol will be launched shortly. One of the goals of this protocol is to increase the percentage of complete resections. An internet survey was conducted amongst pediatric neurosurgeons in Europe to assess their attitude towards complete resections for ependymoma and the acceptance of a surgical review panel or national reference center.

Fourty three pediatric neurosurgeons responded, $71,4 \%$ agreed that complete resection is important for survival in ependymoma, $80 \%$ agreed to a review panel, but not obligatory. The panel should preferably consist of 3-5 members. The advice of the panel should be based on peer group judgment rather than on expert opinion. Five locations and sizes of surgical residues were presented to responders. More than $50 \%$ of responders would perform second look surgery for residues larger than $3 \mathrm{~mm}$ in any location (including floor fourth ventricle and in front of brainstem). Smaller residues were only considered amenable to re-resection when located in the roof of the fourth ventricle, in Luschka or on one lateral side of the brainstem. The result of this survey could help to set up a review panel and define guidelines for resection. To participate in the SIOP protocol a national review panel needs to be installed but consulting should remain voluntary.

\section{Session 6: Neurovascular management}

\section{OP37}

Neurosurgical management in pediatric moyamoya: experience of 139 cerebral revascularizations

Constantin Roder, Florian Ebner, Dubravka Deanovic, Gerasimos Baltsavias, Nadia Khan (Zurich, Switzerland; Tuebingen, Germany)
Objectives: Multiterritorial strokes in the ACA, MCA and PCA (anterior, middle, posterior cerebral artery) vascular distribution are very common in pediatric moyamoya. We present the neurosurgical treatment and postoperative outcome in newly diagnosed patients referred to our Moyamoya Center, University Children's Hospital Zurich.

Material and Method: Forty-five children with newly diagnosed moyamoya angiopathy at a mean age of 7 years (range 1-17 years) underwent preoperative workup and cerebral revascularization between 2011-2015. The female:male ratio was 1.5:1. Correlating clinical symptomatology, number of vascular territories involved on cerebral angiogram and extent of perfusion reserve deficits on H215-O PET with acetazolamide, surgery was tailormade to each individual child.

Results: In total 139 revascularizations were performed collectively in the ACA $(n=51), \operatorname{MCA}(n=76)$ and PCA $(n=12)$ territories. Multiple combined direct and indirect cerebral revascularizations were procedures of choice. A direct EC-IC (extracranial to intracranial) bypass $(n=61$ STA-MCA, 3 STA-ACA) was always performed together with an indirect procedure (EGPMS: encephalo-galeaperiost-myosynangiosis and EDS: encephalo-duro-synangiosis). Indirect technique of EDAS: encephalo-duro-arterio-synangiosis with EGPMS was used only when the donor or recipient vessels were of inadequate caliber and quality for a direct anastomosis $(\mathrm{n}=26)$.

One staged unilateral or bilateral MCA revascularisation was performed in 21 patients.

Two staged surgeries (first MCA followed by ACA) was performed in 23 patients, 3 staged (MCA, ACA, PCA respectively) in 1 patient. There were no immediate perioperative complications. All patients remain stroke free at 3.5 years followup. Systematic Long term follow-up (up to age of 18 years) continues.

Conclusions: Combined direct and indirect revascularizations staged for multiple vascular territories can be safely and effectively performed in pediatric moyamoya.

\section{OP38}

Long-term outcome after multiple burr hole surgery in children with moyamoya angiopathy: a single centre experience in 108 hemispheres

Thomas Blauwblomme, Bertrand Mathon, Olivier Naggara, Manoelle Kossorottof, Maris Bourgeois, Stephanie Puget, Philippe Meyer, Valentine Brousse, Marianne de Montalembert, Francis Brunelle, Michel Zerah, Christian Sainte-Rose (Paris, France)

Importance: Multiple burr hole surgery (MBH) is a simple, safe and effective indirect technique of revascularization in moyamoya angiopathy (MM). However, it is not yet recognized as a first line treatment. 
Objective: The goal of this study is to assess the long-term outcome and perioperative complications in a large singlecenter cohort of children with MM who underwent burr hole surgery.

Design and Setting: Retrospective analysis of consecutive MM children operated in a national reference center for pediatric stroke between 1999 and 2015.

Participants: Sixty-four children (108 hemispheres, median age 7 years) were consecutively treated. Indication of revascularization was: previous stroke or TIA, or rapidly progressing disease on brain MRI and digital subtraction angiography (DSA).

Main Outcomes and Measures: Children were followed by physical examinations and telephone interviews, with any clinical recurrence of stroke or TIA used as the primary endpoint. Surgical mortality and morbidity were documented.

Results: 64 patients were operated (bilateral MBH n=39, unilateral procedure $n=25$ ). At a mean follow-up of 4.2 years and 265.6 patient years, $89.1 \%$ of patients had not suffered from any recurrent stroke or TIA. A second surgery was required in 5 cases after unilateral revascularization, and in 3 cases after bilateral MBH. Mortality associated with the procedure was nil. Postoperative Matsushima angiographic grading was the only predictive factor of ischemic recurrence $(\mathrm{p}=0.036)$.

Conclusion and Relevance: In pediatric MM, multiple burr hole surgery is safe and highly effective in the prevention of stroke or TIA, and has comparable outcomes to other indirect or direct revascularization techniques in children.

\section{OP39}

Cerebral blood flow improvement after indirect revascularization for pediatric moyamoya: a statistical analysis of arterial spin labeling MRI

Thomas Blauwblomme, Herve Lemaitre, Olivier Naggara, Raphael Calmon, Manoelle Kossorottof, Maris Bourgeois, Bertrand Mathon, Stephanie Puget, Michel Zerah, Francis Brunelle, Christian Sainte-Rose, Nathalie Boddaert (Paris, France)

Background: Severity of Moyamoya is generally scaled with conventional angiography, and nuclear medicine. ASL MRI is now acknowledged for the non-invasive quantification of cerebral blood flow (CBF). This study aims at analyzing $\mathrm{CBF}$ modifications with a statistical parametric mapping of ASL MRI in children operated for Moyamoya.

Material and Methods: We included 15 children treated by indirect cerebral revascularization with multiple burr holes between 2011 and 2013. ASL-MRI and T1 sequences were then analyzed under SPM8, according to the general linear model, before and after surgery ( 3 and 12 months). Voxel based analysis was performed at the group level, comparing all sick hemispheres to all normal hemispheres, and at the individual level, comparing each patient to a control group.
Results: Group analysis displayed statistically significant preoperative hypoperfusion in the MCA territory in the Moyamoya hemispheres, and a significant increase of cerebral perfusion in the same territory after revascularization ( $p<0.05$ Family Wise Error corrected). Before surgery, individual analysis showed significant hypoperfusion for each patient colocalized with the angiographic defect on DSA. All but one patient had improvement of CBF after revascularization, correlated with their clinical status.

Conclusion: SPM analysis of ASL MRI offers a non-invasive evaluation of preoperative cerebral hemodynamic impairment, and an objective assessment of postoperative improvement in children with Moyamoya.

\section{OP40}

Clinical features, course and outcomes of pediatric moyamoya

Sara Tho-Calvi, Dominic Thompson, Vijeya Ganesan (London, United Kingdom)

Objectives: To describe clinical and radiological features of children with moyamoya (MM) and examine determinants of outcome.

Methods: Retrospective notes review. Clinical data: demographics, presentation, clinical course, treatment and outcome. Distribution of brain/cerebrovascular disease, and progression, were abstracted from reports. Logistic regression was used to examine associations with outcome.

Results: 71 children (median 5.2y, 48 female) were included. 52 presented with arterial ischaemic stroke [AIS] or transient ischaemic events [TIA]. 26 had a MM risk factor; 5 had a positive family history.

Arteriopathy affected the carotid circulation in 135 hemispheres; 24 patients had posterior circulation involvement. The first brain scan showed acute infarction in 26/135 hemispheres and established infarcts in 72/135. Treatment included antiplatelet drugs (57/71) and revascularisation surgery (75/ 135 hemispheres).

50/71 had recurrent symptoms (17 AIS) within a median of 1 month of initial presentation, of whom 45 went on to have surgery. 26/45 surgical patients had further symptoms, AIS in 3 cases. On re-imaging, further infarcts were seen in 24/112 hemispheres and progression of arteriopathy in $25 / 55$ patients. Outcome was good in 37/69. Children who presented with TIA $(\mathrm{OR}=0.06,95 \% \mathrm{CI}=0.01-0.34)$ or headache $(\mathrm{OR}=$ $0.10,95 \% \mathrm{CI}=0.01-0.69)$ had significantly lower odds of poor outcome than those presenting with AIS. Having controlled for presentation and MM risk factors, posterior circulation involvement was significantly associated with poor outcome $(\mathrm{OR}=6.59,95 \% \mathrm{CI}=1.30-33.45)$.

Conclusions: MM is associated with multiple recurrences, progressive arteriopathy and poor outcome in around half of cases. The latter is less likely with non-AIS presentation and 
more likely if the posterior circulation is involved. Recurrent AIS is rare after surgery but surgery was not a determinant of overall outcome; this could reflect surgical selection criteria or the patients' clinical state at referral.

\section{OP41}

CNS cavernomas in the pediatric age group - A single center experience

Alexandru Tascu, Adrian Barari, Stefan Mircea Iencean (Bucharest, Romania; Iasi, Romania)

Objective: Pediatric CNS cavernous malformations represent a challenging field of research, because of poorly documented studies in literature. Most previous such studies, describe these vascular lesions in both pediatric and adult groups together.

Material and Methods: Our retrospective study analyses the clinical, imagistic, therapeutic and outcome particularities in pediatric patients with CNS cavernomas. The study group included 58 patients under 18 years-old, diagnosed with cavernous malformation, from 1996 to 2015. All the patients were investigated by CT scan and MRI. In some cases a differential diagnose with AVM's was required, and a DSA was performed.

Results: There were 33 males and 25 females, with an average age of 11.4 years-old. Regarding the location, there were 44 supra-tentorial (75.86\%), 10 infra-tentorial (17.24\%), 3 intraorbital $(5.17 \%)$ and one case of spinal cord cavernoma $(1.72 \%) .20$ cases of supra-tentorial locations were frontal lobe, 11 parietal, 7 temporal, 3 thalamic and 3 basal ganglia cases. There were 6 cases (10.34\%) of brain-stem and 4 cases (6.89\%) of cerebellar cavernomas. The most common presenting symptoms in supra-tentorial group were seizures, headache and focal neurological deficits. The most common infra-tentorial onset symptoms were cranial nerve deficits, followed by hemiparesis, numbness and cerebellar symptoms. Visual impairment, oculomotor nerves palsy and exophthalmia were characteristic for intra-orbital location. Hemorrhage was presented as intracerebral hematoma (15 cases), subarachnoid hemorrhage (1 case) and intraventricular bleeding (1 case). Deep location was associated with higher rate of hemorrhage and worse outcome. In our study 3 cases $(50 \%)$ of brainstem, 2 cases $(66.6 \%)$ of basal ganglia and 1 case $(33.3 \%)$ of thalamic cavernous malformations, presented hemorrhage, compared with $28.9 \%$ bleeding cases in superficial locations. Microsurgical resection was performed in 32 of $58(55.17 \%)$ patients. Conservative attitude with clinical and imaging follow-up was decided for 26 patients. The mean follow-up period was 23.44 months. The overall outcome was good, resulting in improved seizure control and less neurological deficits.

Conclusions: Surgery should not be proposed for all lesions. Surgical treatment is considered as choice in cases of symptomatic and hemorrhagic cavernomas (according to localization), and is the only way to prevent re-bleeding and to improve the control of seizures.

\section{Session 7: Craniofacial Surgery}

\section{OP42}

Preoperative and longitudinal complete cognitive evaluation in children operated on for single suture craniosynostoses: early recognition and rehabilitation of selective impaired functions contribute to an improvement of long term neurodevelopmental outcome

Daniela Chieffo, Gianpiero Tamburrini, Irene Bernardini, Luca Massimi, Paolo Frassanito, Concezio Di Rocco, Massimo Caldarelli (Rome, Italy; Hannover, Germany)

Introduction: Children affected by mono sutural synostosis might have an adverse neurodevelopmental outcome, especially concerning visual, cognitive, language and motor domains. The goal of our study was to provide the opportunity to reliably assess longitudinally neurocognitive functions in children operated on for monosutural craniosynostosis and to evaluate the possibility of an early neurorehabilitation programme to prevent possible neurocognitive disorders.

Methods: Formal neurocognitive and neurolinguistic investigations were carried out. Neurocognitive investigations consisted of Griffith Mental Developmental Scale (GMDS), the Wechsler preschool children Intelligence Scale-III (WIPPSI III), various aspects of neuropsychological function, i.e. visual attention (LEITER Scale), verbal immediate and recent memory, were investigated by means of the Nepsy II. Neurolinguistic evaluation was performed by phonological and lexical examination (naming and comprehension-TFL). Visualperceptual skills and visuo motor integration was evaluated with the Beery test.

Results and Conclusions: Between January 2008 and December 2015223 children affected by single suture craniosynostoses have been evaluated and treated for a single suture craniosynostosis at the Catholic University Medical School, in Rome, Italy.

78 of them (30 affected by scaphocephaly, 30 by anterior plagiocephaly and 18 by trigonocephaly), have completed a selective neuro cognitive evaluation in four longitudinal time periods: T0: preoperative evaluation; T1: between 24 and 36 months of life; T2 between 60 and 72 months and T3 at the beginning of their first elementary school class start.

At T1 Global IQ was in the normal range in 68 children (87\%) and borderline in $10(13 \%)$; selective neurocognitive evaluation showed visual attention deficits and motor hypercinesia in $23 / 30(77 \%)$ children affected by scaphocephaly, $18 / 30(60 \%)$ children affected by anterior plagiocephaly and 7/18 (39\%) children affected by trigonocephaly (Group A). Moreover $18 / 30$ children with scaphocephaly $(60 \%), 12 / 30$ children 
affected by anterior plagiocephaly (40\%) and 10/18 children affected by trigonocephaly (55\%) showed expressive language disorders (Group B).

Children in Group A were sent to a psychomotor rehabilitation programme selectively directed at the improvement of attention time and behaviour; children in Group B were sent to a global psychomotor rehabilitation programme and a specific logopedic programme. At T3 (mean T3 time $=6.4$ yrs), global IQ impairment improved in all but 4 children (4/68 cases, $5 \%$ ). Attention and language deficit showed a progressive and longitudinal improvement, persisting respectively in $40 \%$ and $26 \%$ of the cases. Reading and writing impairments in particolar persisted in a distinctive minority of them, without significant relation with the craniosynostosis type. These results were significantly improved compared with a previously studied and matched group of children who were evaluated only at diagnosis and at a final mean term of $14.3 \mathrm{yrs}$. In conclusion a longitudinal preoperative and postoperative neurodevelopmental evaluation programme might improve the long term cognitive outcome of children affected by single suture craniosynostoses.

\section{OP43}

Comparison of piezosurgery and conventional instruments in cranioplasty with fronto-orbital advancement

Markus Martini, Andreas Röhrig, Martina Messing-Jünger (Bonn, Germany; Sankt Augustin, Germany)

Object: In order to compare possible advantages and disadvantages of the piezosurgery device with standard bone cutting instruments in realizing fronto-orbital advancement for craniosynostosis surgery a prospective clinical study was performed.

Material and Methods: In a controlled prospective study a piezosurgery device was tested versus a standard oscillating saw for performing bone cuts in fronto-orbital advancement as part of cranioplastic surgery in craniosynostosis. Handling features, cutting time and dural or periorbital lesions as well as peroperative parameters like swelling, $\mathrm{Hb}$, blood loss, CRP and bone healing assessed during osteosynthesis removal were evaluated. Additionally standardized pain protocols were implemented (NIPS, KUSS).

Results: In 34 infants with trigonocephaly (23), anterior plagiocephaly (8), brachycephaly (2), and syndromic craniosynostosis (2) fronto-orbital advancement was performed. Mean age at surgery was 9.5 months. Piezosurgery (18) was compared to standard oscillating saw (16) technique. No significant differences regarding dural and periorbital lesions, blood loss, $\mathrm{Hb}$ or postoperative swelling and pain levels could be observed. Osteotomy took longer with piezosurgery, whereas postoperative CRP increase was higher with standard osteotomy. No infections or wound healing disorders have been observed in boths groups.
Conclusion: Piezoelectric surgery is a sufficient osteotomy method in cranioplasty but requires the same precautions as conventional operating procedures and leads to a slightly extended surgery time.

\section{OP44}

Comparison of early minimal invasive cranioplasty without regular helmet therapy compared to standard open cranioplasty in sagittal synostosis

Martina Messing-Jünger, Sandra Kunze, Andreas Röhrig, J. Otten, Markus Martini (Sankt Augustin, Germany; Bonn, Germany)

Object: In order to compare early minimal invasive cranioplasty without regular helmet therapy to standard open cranioplasty a prospective study was performed.

Material and Methods: In a prospective observational study pre- and postoperative optical scan data (3, 6 and 12 months postop) have been used to assess cranial index (CI) and other morphometric parameters. Helmet therapy was only indicated in cases of secondary deformities like positiong plagiocephaly etc. Patients of both groups have been positioned postoperatively in a straight midline position during sleep.

Results: 120 infants with sagittal synostosis have been evaluated. Open standard surgery was performed in 51 (11 f/ 40 $\mathrm{m})$ and early minimal invasive cranioplasty in $69(17 \mathrm{f} / 52 \mathrm{~m})$. Mean age in the standard group was 211 days/ 7 months and 92 days/ 3 months in the minimal invasive group. Adjuvant helmet treatment was indicated in 2 standard cases and 11 minimal invasive cases. In the standard group CI changed from 71 to 79 , in the minimal invasive group from 71 to 77 at the end of the observation time (12 months). Skull volume as well as head height and shape in general were also normalized in both groups in $80-90 \%$.

Conclusion: Consequent helmet therapy after minimal invasive cranioplasty is not necessary in order to maintain a sufficient normalization of CI and head shape in general.

\section{OP45}

A novel, non-bandeau technique for fronto-orbital remodeling in synostotic trigonocephaly and plagiocephaly: report of 328 cases

Olivia Thornett, Gregory James, Justine O'Hara, Owase Jeelani, David Dunaway, Richard Hayward (London, United Kingdom)

Introduction: Fronto-orbital remodeling (FOR) is an operation for correction of deformities caused by metopic or unicoronal synostosis. The standard FOR technique (developed by Marshac and Renier) is via removal, remodeling and replacement of the supraorbital "bandeau"- involving multiple osteotomies and implants. We developed an alternative technique, using an optimal bony contour selected from a 
posterior portion of the frontal craniotomy to replace the deformed supraorbital region (which is resected). No implants, other than suture and dental wire, are used. We describe this technique and present our surgical series.

Methods: Retrospective electronic database review of all cases since adoption of this technique in 1995.

Results: We identified 328 consecutive FOR operations in 322 children. There were 169 boys and 153 girls, with 167 metopics and 155 unicoronals. Our institution introduced prospective adverse event recording in 1999 - in the 284 cases since, there were 13 complications $(4.7 \%)$. There was 1 mortality due to acute post-operative extradural haematoma. There were 8 surgical site infections, 2 CSF leaks and 2 miscellaneous complications. Of the 30 most recent cases, 19 required transfusion of blood products $(63 \%$, maximum 1 donor exposure), mean theatre time (including anaesthesia) was 214.5 minutes (range 144-283) and mean post-op hemoglobin was $105.37 \mathrm{~g} / 1$ (range 53-132, a mean drop of 15 from preoperative levels). Mean length of hospital stay was 4 postoperative days. Regarding surgical results, $6 / 322$ (2\%) of children required revisional surgery at our institution. Of the most recent 114 patients, parental opinions on results were available for $111.92(83 \%)$ of parents were completely happy with the appearance, $17(15 \%)$ had minor concerns and $2(2 \%)$ had major concerns (one due to uncorrected orbital dystopia and the other due to frontotemporal hollowing).

Conclusions: We describe a safe and efficacious alternative technique for FOR in children with metopic or unicoronal synostosis.

\section{OP46}

3D handheld optical scanning for quantitative evaluation of head shape changes following front-orbital remodeling for trigonocephaly

Ozge Goktekin, Naiara Rodriguez-Florez, Jan Bruse, Alessandro Borghi, Freida Angullia, Maarten Koudstaal, Sylvia Schievano, Justine O'Hara, David Dunaway, Owase Jeelani, Gregory James (London, United Kingdom; Rotterdam, the Netherlands)

Introduction: Objective assessment of fronto-orbital remodeling (FOR) surgery outcomes for correction of trigonocephaly traditionally requires pre- and post-operative CT scans. However, CT involves ionizing radiation and requires general anesthesia (GA) in small children. 3D optical surface imaging is a potential alternative that does not involve radiation and is non-invasive. We hereby describe our positive experience with applying this technique to patients who underwent FOR for trigonocephaly with the hypothesis that it can be applicable to other craniofacial operations.

Methods: Eight patients (6 male; mean age 18.1 \pm 3.3 months) undergoing FOR for trigonocephaly were recruited. Optical scans were acquired pre- and post-operatively in theatre, using a 3D handheld camera (M4D scanner, Rodin4D). The scans were cut by a plane defined by the nasion and the left and right crus of helix, and a second plane at $120^{\circ}$ from the first to capture the remodeled surface. 3D scans were used to measure i) frontal angle, ii) interfrontoparietal-interparietal ratio, a ratio which describes the narrowing of the fronto-temporal region, and iii) volume. Pre- and postoperative measurements were compared with Wilcoxon signed ranks test. Differences in surface distances between pre- and postoperative scans were visualized as $3 \mathrm{D}$ color maps.

Results: The frontal angle increased in all patients postoperatively ( $\mathrm{p}=0.012$ ) with a mean increase of $12 \pm 3 \%$. The interfrontoparietal-interparietal ratio increased $(p<0.02)$ with a mean increase of $16 \pm 7 \%$. Volumetric measurements showed an increase $(\mathrm{p}=0.012)$ with a mean change of $10 \pm 6 \%$. The color maps show that the metopic ridge decreased by $3 \mathrm{~mm}$ and the fronto-parietal area widened by $5 \mathrm{~mm}$.

Conclusion: $3 \mathrm{D}$ optical surface imaging is an effective tool to assess head shape change following FOR, with the advantages of not using ionizing radiation or GA. Results suggest that FOR successfully improves trigonocephaly in the analyzed patients. This technique could be used to quantitatively evaluate the outcome of other craniofacial interventions.

\section{OP47}

Computer assisted virtual planning and surgical template fabrication for fronto-orbital advancement: pilot evaluation of outcome

Jehuda Soleman, Benito Benitez, Joerg Beinemann, Christian Schneider, Valerie Oesch, Christoph Kunz, Raphael Guzman (Basel, Switzerland; Aarau, Switzerland)

Aim: We applied our previously published technique, using computer-assisted design (CAD) and computed assisted manufacturing (CAM) for the fabrication of individualized 3D printed surgical templates for fronto-orbital advancement surgery in craniosynostosis surgery. This pilot study evaluates the outcome using this novel technique.

Methods: Twelve patients underwent a fronto-orbital advancement surgery using the novel CAD/CAM technique. Three patients had a uni-lateral and one patient a bi-lateral coronal synostosis and eight patients a metopic synostosis. Virtual surgical planning was performed using SurgiCase ${ }^{\circledR}$ (CMF, v5.0, Materialise, Belgium). A novel algorithm was developed combining virtual mirroring techniques and superposition of age-matched normative 3D pediatric skull models. Surgical templates were fabricated using a 3D printer, based on the virtual plan. Bi-frontal craniotomy and the osteotomies for the orbital bandeau were performed based on the sterilized $3 \mathrm{D}$ templates. The remodeling was then done placing the bone plates within the negative 3D templates and fixing those using absorbable PDLLA plates and screws (SonicWeld, KLS Martin, Tuttlingen, Germany). The postoperative outcome 
was assessed using 3D photographs of the patients and comparing them to the pre-surgical planning.

Results: The mean age of the patients was 5.4 months and mean operation time was 286.6 minutes. No surgical related complications occurred. The cutting and positioning of the $3 \mathrm{D}$ surgical templates proved to be very accurate, easy to use as well as reproducible and efficient. All patients showed a satisfying correction of cranial deformity postoperatively and at follow up. Additional intra- and postoperative measurements (e.g. blood loss, hospitalization time) and the patients' outcome based on postoperative $3 \mathrm{D}$ photography will be presented.

Conclusions: Computer assisted virtual planning and 3D template fabrication for fronto-orbital advancement surgery, leads to reconstructions based on standardized measurements, precludes subjective remodeling and seems to have an overall good outcome. An ongoing prospective study with a planned follow-up of 5 years will be conducted at out institution for further evaluation of the outcome of this novel technique.

\section{OP48}

Radiation-free 3D head shape and volume evaluation after endoscopically assisted craniosynostosis surgery

Guido de Jong, Manon Tolhuisen, Jene Meulstee, Thomas Maal, Hans Delye (Nijmegen, the Netherlands)

Introduction: The use of radiation-free imaging after craniosynostosis surgery is hindered by the lack of consistent markers as there are no bony landmarks to rely on when comparing sequential images. Using the computed cranial focal point (CCFP), it is possible to calculate the position of the sella turcica, allowing correct superposition of sequential images. We used this technique for mean volume and shape change evaluation of the head based on 3D Photos after craniosynostosis surgery.

Methods: We performed a head shape and volume evaluation on 3D Photos $(\mathrm{n}=84)$ of children that underwent endoscopically assisted trigonocephaly craniosynostosis surgery with helmet therapy between December 2010 and October 2015. The 3D Photos were grouped in pre-surgery, 6, 12, 18, 24, and 36-48 months post-surgery. We used the CCFP and nasion to orient the 3D Photos and evaluate the mean shape and volume above the sella-nasion plane. We calculated the anterior to complete volume ratio of the mean heads and performed a shape evolution analysis.

Results: The mean anterior volumes of the head evolve from $300 \mathrm{ml}$ pre-surgery to $607 \mathrm{ml} \mathrm{36-48} \mathrm{months} \mathrm{post-surgery.} \mathrm{The}$ mean ratio between the anterior volume and complete volume starts at $31.0 \%$ and increases until 12 months to $34.4 \%$. In the first 18 months there is a predominant increase in the forehead, area of the resected metopic suture. Between 18 and 24 months the growth is focused at the orbital rims growing in an anterior direction. From 18 months till 36-48 months the head grows uniformly in all areas.

Conclusion: Using a novel technique we were able to perform mean shape and volume evaluation of the head after craniosynostosis surgery. There is an increase in the ratio of the anterior to complete head over time and a predominant growth in the area of surgery until 18 months post-surgery.

\section{OP49}

The computed cranial focal point

Guido de Jong, Thomas Maal, Hans Delye (Nijmegen, the Netherlands)

Introduction: Stereophotogammetry is used for monitoring skull development after craniosynostosis repair. Lack of clear fixed reference points complicate longitudinal comparison of 3D-photos. Therefore we developed the "Computed Cranial Focal Point" (CCFP) in which all cranial surface normals tend to intersect.

Methods: The CCFP was calculated in segmented 3D CTscans of 34 adult subjects using Matlab. The robustness of the CCFP calculation was tested comparing the error between the calculated and known focal points in predefined shapes. Finally we used the CCFP in a clinical case to correlate the CT data with 3D-photo data.

Results: CCFP calculation showed to be hardly influenced by incomplete or deformed surface data, maintaining a submillimeter accuracy. The average position relative to the sella turcica of the CCFP-skull was at $(0.0,29.9,18.6) \mathrm{mm}$ and for the CCFP-skin at $(0.1,27.9,20.0) \mathrm{mm} .95 \% \mathrm{CI}$ was less than mean $\pm 2.7 \mathrm{~mm}$ in each direction. The mean difference between the CCFP-skull and CCFPskin is $(0.1,-2.0,1.4) \mathrm{mm}$ with a maximum $95 \% \mathrm{CI}$ equal to mean $\pm 0.6 \mathrm{~mm}$ in each direction. Using the CCFP to correlate the skin from a 3D-photo and the segmented skin from a CT-scan results in an overlay with a mean average error of $0.78 \mathrm{~mm}$.

Conclusion: The CCFP calculation is a robust method to define a fixed reference point relative to the sella turcica based on skin or cranial bone surface. The CCFP can be used to correlate 3D photo to CT-scan data or for longitudinal comparison of 3D-photos.

\section{OP50}

Management of positional plagiocephaly with cranial helmet in a series of 2188 children treated between 1991 and 2013. Our experience

T. Picart, Alexandru Szathmari, Carmine Mottolese (Lyon, France)

Introduction: Positional plagiocephaly incidence constantly increases since 1992 as pediatricians advise supination or side sleep in young infants. We conducted a retrospective 
epidemiological study to assess the results of management of positional plagiocephaly with a cranial helmet $(\mathrm{CH})$.

Material and Methods: We reviewed 2188 patients (75\% M and 25\% F) treated between 1991 and 2013. We noted sex, age, type of plagiocephaly, lengthof treatment and morphologic parameters at baseline and after treatment: head circumference, right and left tragus-lateral canthus distance, right and left tragus-corner of the mouth distance, cranial index (CI) and Cranial Diagonals Difference (CDD). Children presented unilateral deformational plagiocephaly (UDP) were classified into 3 groups by severity. Measures were analyzed to assess and to quantify the effectiveness of cranial helmet.

Results: For children withbrachycephaly, average Cranial Index (CI) improved from 101.72 to 96.7 at the end of the treatment. For UDP, there was $2.5 \%$ mild (CDD $<5 \mathrm{~mm}$ ) $20 \%$ moderate $(5 \mathrm{~mm}<\mathrm{CDD}<10 \mathrm{~mm})$ and $77.5 \%$ severe plagiocephaly (CDD $>10 \mathrm{~mm})$. At the end, much more children in were in mild (40.2\%) or moderate-plagiocephaly groups (44.3\%) and less in the third group (15.5\%). Initial median CDD was $15 \mathrm{~mm}$ and final was of $6 \mathrm{~mm}$. Facial asymmetry (13.7\%) resolved in $66.7 \%$ of cases. Eight children $(0.2 \%)$ were operated. Average duration of treatment was of 199 days. Side effects were low with erythema (1.6\%) or dermabrasions $(0.6 \%)$.

Conclusion: The cranial helmet is a simple and well-tolerated treatment. We show the $\mathrm{CH}$ modifies the cranial index and oblique asymmetries. Its success implies a good collaboration between parents and orthoptist, physiotherapist and doctors. It must be applied as early as possible. Nevertheless, there remain many controversies in the literature concerning longterm cosmetic and functional outcomes.

\section{Session 8: Intraoperative neuromonitoring}

\section{OP51}

Intraoperative neurophysiology in pediatric supratentorial surgery: experience with 59 cases

Akiva Korn, Haggai Benvenisti, Muna Jubran, Yifat BitanTalmor, Perla Ekstein, Shlomi Constantini, Jonathan Roth (Tel Aviv, Israel)

Introduction: The utilization of intraoperative neurophysiology (ION) to map and assess various functions in brain tumor and epilepsy surgery is well documented and commonplace in the adult setting. It is less described in the pediatric setting due to the relatively low incidence of relevant pathologies, as well as relative difficulty to implement these methods for physiological reasons. The applicability has yet to be established as feasible or beneficial, and optimizations of current techniques are not known.

Methods: Retrospective analysis was undertaken for the following variables in all pediatric supratentorial surgery utilizing ION of the motor system over a period of 9 years: preoperative and postoperative motor deficits, extent of resection, monitorability and mapability of sensory-motor evoked potentials, location of lesion, age and monitoring alarms. Intraoperative findings were correlated with short and long term postoperative clinical outcome. The monitoring impact on surgical course was evaluated on a per case basis.

Results: Data of 59 patients were analyzed (avg age 94.1 \pm 58.6 months). Deep lesions (in proximity of the pyramidal fibers) consisted of $18.5 \%$ of the total group, superficial lesions $54.2 \%$, lesions with both deep and superficial components $22.0 \%$ and ventricular $5.1 \%$. Monopolar mapping of the motor cortex was significantly more successful than bipolar mapping ( $84.6 \%$ vs. $28.1 \%$ of trials respectively), the youngest age mapable was 3 vs 154 months for either method respectively. Successful mapping of the primary sensory cortex correlated with increasing age, and was found at 107.4 \pm 59.5 mo., all older than 6 mo. 23 patients had full clinical and ION motor correlations. The sensitivity, specificity, PPV and NPV values for verified dcMEP changes on immediate postoperative clinical condition were $45 \%, 92 \%, 83 \%$ and $65 \%$ respectively. The predictability of a postoperative motor deficit inversely correlated with the scrtMEP value, peaking at $72 \%$ in the sub $3 \mathrm{~mA}$ threshold group. Low scrtMEP impacted surgical decision making in 4 patients, cortical motor mapping altered the corticectomy entry point/extent of resection in 9 cases, and a dcMEP monitoring alarm altered the course of surgery in 11 cases.

Discussion and Conclusion: This paper describes the largest cohort of monitored pediatric supratentorial brain surgeries to date. The relative difficulty of sensory-motor mapping and monitoring correlates with the timeline of maturation of these systems. ION is applicable in the pediatric population; however with certain limitations depending mainly on age. Yet when successful, ION has a positive impact on surgical decision making, ultimately providing an added element of safety to these patients.

\section{OP52}

\section{Brain stem safe entry zone in child}

Sergio Cavalheiro, Kan Yagmurlu, Marcus da Costa, Jardel Nicácio, Thiago Rodrigues, Feres Chadad-Neto, Patricia Dastoli (Sao Paolo, Brazil; Gainesville FL, USA)

Introduction: Brainstem surgeries are among the most difficult in neurosurgery, especially for pediatric neurosurgeons, as brainstem tumors are more frequent in childhood than in adult life. Although imaging with tractography has evolved significantly, they still do not show the nuclei and nerve pathways inside the brainstem. Accordingly, the aim of this study is to present the anatomic landmarks in the brain stem "safe entry zone" used in children.

Methods: Retrospective review of patients diagnosed with brain stem tumor operated under the age of 18 years from 
1991 to 2011 at Neurosurgical Service and Pediatric Oncology Institute of the Federal University of São Paulo. Categorization of each safe zone and approach used to reach the brain stem lesion.

Results: We identified 207 patients operated, ages ranged from 8 months to 18 years, with a mean age of 10 years. The tumors were distributed as follow: $40.5 \%$ in the midbrain, $34.8 \%$ in the pons and $24.7 \%$ in the bulb. The brain stem was divided into seven zones: anterior, median and posterior midbrain; anterior and posterior pons; anterior and posterior bulb. Twelve "safe entry zones" were identified: perioculomotor, the lateral mesencephalic sulcus, infracollicular access, supracollicular access, peritrigeminal, suprafacial, interfacial, infrafacial, lateral sulcus limitans, periolivary, and the posterior median sulcus. A new safe entry zone has been used in three of our patients, the "supratrigeminal" zone, for approaching anterior and superior pontine lesions. The operative mortality seen in the first 2 months after surgery was $1.9 \%$ (four patients) and the morbidity rate was $21.2 \%$.

Conclusion: Anatomic knowledge of intrinsic and extrinsic brainstem structures, in association with a refined neurosurgical technique assisted by intraoperative monitoring, and surgical planning based on Magnetic Resonance Imaging (MRI) and tractography, have allowed for wide resection of brainstem lesions with low mortality and acceptable morbidity rates.

\section{OP53}

Neuromonitoring during de-tethering cord procedures in infants: the bulbocavernous reflex in total removal lipoma surgery

Roberto Cordella, Giorgio Selvaggio, Elena Beretta, Stefania Bova, Marika Furlanetto, Alessandra Erbetta, Laura Grazia Valentini (Milan, Italy)

Occult spinal dysraphism may cause progressive neurological deterioration by a condition called tethered cord syndrome, namely an incorrect anatomic position of the conus. This might lead to sensorylmotor deficits, orthopedics, anorectal and urodynamic (UD) abnormalities. Recently, the surgical trend is to obtain radical lipoma removal to prevent retethering with growth. This surgery is challenging and intraoperative neurophysiological monitoring (IOM) is pivotal to prevent iatrogenic nerve injuries. Multimodal IOM comprises EMG, tMEP, SSEP and the bulbocavernous reflex (BCR). The BCR reflects the functional integrity of sacral sensorylmotor nerve roots, (from S2 to S4). Pudendal nerves' sensory fibers are the afferent pathway, and motor fibers to pelvic floor or external anal sphincter muscles are the efferent pathway. This review concerns 40 infants (age range: 6 months to 13 years) operated on to remove lipoma and de-tethering with the aid of IOM (20 with BCR 120 NO-BCR). The "radical removal" technique was applied in 30 children, 20 had BCR and 10 didn't. The dorsal portion of the penis or clitoris is stimulated and recordings were obtained from the external sphincter muscles, bilaterally. Stimulation parameters may range from single pulse $(1 / 5 \mathrm{~Hz})$, double pulse (ISI $3 \mathrm{~ms}$ ) train, to the train of five method with pulse width of $0.1 \backslash 0.2 \mathrm{~ms}$. UD evaluation comprises cystometrogram and EMG of pelvic floor, abdominal pressure for subtracted detrusorial pressure and urinary tract ultrasound. BCR was recorded in all but 2 patients. The post-op incidence of new UD symptoms were $20 \%$ in the NO-BCR group and $11 \%$ in the BCR group. BCR is feasible to be recorded in pediatric population during sacral plexus neurosurgical procedures, although it has not been recorded in 2 patients with severe pre-op UD symptoms. BCR has the great advantage to detect conduction block at both the peripheral and central levels and might impact the rate of post-operative urodynamic symptoms.

\section{Session 9: Hydrocephalus}

\section{OP54}

Repeat endoscopic third ventriculostomy in pediatric patients: the Dutch experience

Gerben Breimer, Ruben Dammers, Peter Woerdeman, Hans Delye, Dennis Buis, Eelco Hoving (Amsterdam, the Netherlands; Rotterdam, the Netherlands; Utrecht, the Netherlands; Nijmegen, the Netherlands; Groningen, the Netherlands)

Introduction: Although success rates of endoscopic third ventriculostomy (ETV) are quite high, some patients will eventually develop recurrent symptoms of hydrocephalus. The optimal treatment for these patients is not clear; either repeat ETV (re-ETV) or divert cerebrospinal fluid by means of a shunt.

Objective: To assess the effectiveness of re-ETV in pediatric hydrocephalic patients and provide validity evidence for the ETV success score (ETVSS) for re-ETV.

Methods: Retrospective data of all consecutive 90 pediatric patients undergoing re-ETV in six neurosurgical centers in the Netherlands were collected. We analyzed influence of age, presence of arachnoid membranes, closure of ventriculostomy site, usage of extra-ventricular drain (EVD) and lumbar punctures postoperatively on success rates. Predictive adequacy of ETVSS was tested by means of an unpaired $t$ test, HosmerLemeshow "goodness-of-fit" test, and c-statistic.

Results: At 6 months 61 (68\%) of 90 patients were successfully treated with re-ETV. At a median observed follow-up of 4.7 year (IQR 2.2 to 7.5 ) there was a success rate of $50 \%$. Younger age at re-ETV, presence of prepontine arachnoid membranes and usage of an EVD were negatively associated with chances of success. The ETVSS for re-ETV was significantly higher for successful cases; 66 (SD 15.6) vs. 77 (SD 
12.6), $p<0.001$. The Hosmer-Lemeshow was $p=0.30$ and the c-statistic 0.73 (95\% CI 0.63-0.84).

Conclusions: Re-ETV seems to be as safe and effective as initial ETV and the factors related to the success of re-ETV are similar to those related to success of initial ETV (e.g. patient age and etiology of hydrocephalus). Presence of prepontine arachnoid membranes and use of EVD also negatively influence chance of success. The ETVSS adequately predicts chances of success of re-ETV and can be used for patient selection.

\section{OP55}

Zika virus associated microcephaly: a threatening outbreak imposing differential diagnosis with neurosurgical diseases

Eduardo Jucá, André Pessoa, Erlane Ribeiro, Vivian Maria Mota, Pedro Hugo Gouveia, Renata Viana Jucá, Samantha Nunes (Fortaleza, Brazil)

Introduction: Recently, an emergent flavivirus transmitted by mosquitos arrived in Brazil, possibly during the 2014 soccer world cup displacements: zika virus (ZV). The infection, which causes usually a mild febrile disease, was associated in pregnant women to high incidence of microcephaly in newborns. Aim of this study is to describe the outbreak focusing on differential diagnosis with neurosurgical conditions.

Methods: Report of one year casuistic of ZV associated microcephaly (ZVAM) outbreak in Brazil. Report of illustrative cases simulating pediatric neurosurgical diseases.

Results: Until January 9th 2016, 3050 cases of microcephaly were registered (42 deaths), twenty fold the average incidence. Limit head circumference (HC) to diagnosis was $32 \mathrm{~cm}$. Computer tomography (CT) scans show brain atrophy and spread calcifications. Time coincidence and report of symptoms by many mothers lead to an association with $\mathrm{ZV}$. This was confirmed by virus detection in two authopsies. Our pediatric neurosurgery service received two cases mimicking neurosurgical diseases.

Case 1: a 28 years old woman at the 26th week of pregnancy was referred due to ultrasound finding of hydrocephalus in the fetus. A fetal magnetic ressonance showed ventricular enlargement as result of brain atrophy. Delivery ocurred at 38th week. HC was $31 \mathrm{~cm}$, morphology and CT presented the features of zvam.

Case 2: A 22 days old male newborn was referred due to suspicion of craniostenosis. The anterior fontanell was closed. $\mathrm{HC}$ was $30 \mathrm{~cm}$. Clinical aspect and CT scan were compatible with zvam.

Both mothers reported symptoms of $\mathrm{ZV}$ fever in the first months of pregnancy. Assesment by the neurorehabilitation team demonstrated severe disability.

Conclusions: ZV associated microcephaly is a threatening and rapidly spreading outbreak with high potential for mortality and morbidity. Neurological manifestations are devastating and long term disability is expected. Pediatric neurosurgeons must be aware to identify these cases and make differential diagnosis with neurosurgical diseases.

\section{OP56}

International Infant Hydrocephalus Study (IIHS): initial results of a prospective, multicentre comparison of endoscopic third ventriculostomy (ETV) and shunt for infant hydrocephalus

Abhaya V. Kulkarni, Shlomi Constantini, Spyros Sgouros (Toronto ON, Canada; Tel Aviv, Israel; Athens, Greece)

Introduction: The IIHS is an international, prospective, multicentre study to compare ETV and shunt in infants $(<24$ months old) with symptomatic triventricular hydrocephalus from aqueductal stenosis. Recruitment started in 2004 and, here, we present the first results of IIHS.

Methods: IIHS utilized a prospective comprehensive cohort design, which contained both a randomized and nonrandomized arm. Patients received either an ETV or shunt, based on randomization or parental preference. Patients were followed prospectively for time to treatment failure, defined as the need for repeat CSF diversion procedure (shunt or ETV) or death due to hydrocephalus. Survival analysis was used to compare time to failure for ETV versus shunt. The trial was registered at clinicaltrials.gov (NCT00652470).

Results: A total of 158 patients met eligibility criteria (median age at surgery 3.6mths, IQR 1.6-6.6 mths) across 27 centres in 4 continents. Since only 52 patients (32.9\%) were randomized, all 158 patients were analyzed together (115 ETV, 43 shunt). Actuarial success rates for ETV vs shunt at 3, 6, and 12 months were: $68 \%$ vs $95 \%, 66 \%$ vs $88 \%$, and $66 \%$ vs $83 \%$. The 6 month ETV success rate of $66 \%$ was slightly higher than would have been predicted by the ETV Success Score (57\%).The hazard ratio for time to treatment failure favored shunt over ETV (3.17, 95\% CI 1.45-6.96, $\mathrm{p}=0.004)$, after adjusting for age at surgery, history of previous hemorrhage or infection, continent, and randomization status. Patients younger than 6 months of age appeared to do relatively worse with ETV than older patients.

Conclusions: The IIHS has provided the first prospective direct comparison of ETV and shunt for infant hydrocephalus. These initial results suggest that shunting has a superior success rate compared to ETV, although the success rate for both was relatively high. This patient cohort continues to be followed and we will await the results of the important primary outcome of health status at 5 years of age.

\section{OP57}

Shunt-assistants with fixed versus adjustable opening pressures: comparison of clinical parameters in the course of the treatment of pediatric hydrocephalus 
Ines Hallmann, Christoph A. Tschan, Alexandra Huthmann, Wolfgang Wagner (Mainz, Germany; Meppen, Germany)

Objective: The overdrainage of shunts in the treatment of pediatric hydrocephalus is -in the long term- one of the most frequent and most challenging complications. Since many years, anti-gravity valves are available to treat overdrainage. We studied retrospectively possible differences in the course of hydrocephalus treatment when using shunt-assistants with fixed opening pressures ( $\mathrm{SA} ; 10-35 \mathrm{~cm} \mathrm{H}_{2} \mathrm{O}$ ) versus shuntassistants with postoperatively adjustable opening pressures (proSA; 0-40 cm $\mathrm{H}_{2} \mathrm{O}$ ) (Miethke, Potsdam, Germany).

Methods: The data of 46 children, aged 0-16 years and operated on in the Department of Pediatric Neurosurgery Mainz between 2003 and 2012, were evaluated. The studied parameters were the type of shunt-assistant (SA versus proSA), the time of recovery from complaints and the frequency of necessary secondary operations.

Results: In 27 children, a shunt-assistant with fixed opening pressure (SA) and in 19 patients, a shunt-assistant with postoperatively adjustable opening pressure (proSA, both devices Miethke, Potsdam, Germany) was implanted. From the 27 children with SA, 15 (55\%) had to undergo a revision surgery (change of the valve) because of ongoing slit ventricle problems. In the 19 cases with proSA, the opening pressure had to be a adjusted in the postoperative period up to 9 times per patient (average of adjustments during the first year: 2.3 per patient), but no revision surgery was necessary. The mean time until recovery from complaints was 6 years in SA patients and 2 years in proSA patients.

Conclusion: The results confirm the a priori plausible assumption that the frequency of necessary revision surgeries and the recovery time are markedly lower when using a postoperatively adjustable shunt-assistant. In particular the reduction of secondary surgeries overweighs the primarily higher costs of adjustable shunt assistants.

\section{OP58}

Reduction in primary and revision shunt infection rate after introduction of a mandatory shunt perioperative protocol: a single institution retrospective cohort study Osama Omrani, Jody O'Connor, Gregory James (London, United Kingdom)

Introduction: Shunt infection remains a major source of morbidity in pediatric neurosurgical practice. Interest is growing in the use of standardized protocols to reduce infection. In 2012, our institution introduced a mandatory shunt protocol, detailing pre-, intra- and postoperative care.

Methods: We performed a retrospective cohort study using electronic database interrogation. We identified consecutive patients undergoing permanent shunt operations in 2 study periods, each consisting of 36 months -one immediately prior to and the other immediately after the adoption of the protocol as mandatory unit policy (July 2009-June 2012 and July 2012June 2015). Permanent shunt operations were subdivided into 1) primary shunt insertions, 2) shunt revisions and 3 ) internalizations of external CSF drainage. Absolute and relative risk reduction (ARR/RRR) and chi square statistics were calculated.

Results: 962 operations in 546 children were identified (529 pre-protocol, 433 post-). Age was similar in the 2 groups (pre, mean 5.1 years, range $0-20$; post, mean 4.2 years, range $0-18$ ). Overall infection rate decreased from 6.5\% (32 infections in 529 cases) before the introduction of the protocol to $4.8 \%(21 / 433)$ whilst the protocol was in place $(\mathrm{P}=0.14$; ARR $1.7 \%$; RRR $23.2 \%$ ). For primary shunt insertions, the infection rate reduced from 4.1\% (8/193) to $2.5 \%(4 / 160)(\mathrm{P}=0.26$; ARR $1.6 \%$; RRR $39.1 \%$ ). For shunt revisions, there was a decrease from $6.5 \%$ $(16 / 247)$ to $4.3 \%(9 / 210)(\mathrm{P}=0.17$; ARR $2.2 \%$; RRR $34 \%)$. For internalizations there was a small increase in infection rate -from $10.4 \%(8 / 87)$ to $12.7 \%(8 / 63 ; \mathrm{P}=0.6$, ARR $-2.3 \%$, RRR $-22 \%$ ).

Conclusions: Following introduction of the protocol, there was a drop in infections following primary shunts and revisions. The lack of effect in the internalization group suggests our current protocol is inadequate for these cases. In line with previous studies we support introduction of shunt protocols for primary and revision shunts in pediatric neurosurgical units.

\section{OP59}

Ventriculo-subgaleal shunt in preterm infants. Surgical experience

Magda Garzon, Mandeep Kang, Kirin Sandhu, Dolin Baghawatti, Kristian Aquilina, Gregory James, Dominic Thompson (London, United Kingdom)

Introduction: Intraventricular haemorrhage (IVH) and its resulting post-hemorrhagic hydrocephalus $(\mathrm{PHH})$ is the most common neurosurgical problem in preterm neonates and often managed with a temporizing CSF drainage device. Since 2012, our institution has used ventriculosubgaleal shunts (VSGS) as the standard temporizing device. We report our surgical series. Methods: Electronic database and casenote review of consecutive infants requiring VSGS (January 2012 - December 2015). Results: During the study period, 23 infants required VSGS. Mean follow-up was 517 days (range 35-672). Mean gestational age and weight at the time of VSGS was 31.39 weeks (range 27-37, SD 2.8) and $1190 \mathrm{~g}$ (range 758-1790 g, SD 282). Co-morbidities included: $70 \%$ respiratory distress, $87 \%$ suspected sepsis, $52.2 \%$ chronic lung disease, $13 \%$ coagulation abnormalities and 91\% other systemic illnesses. Most infants $(95 \%)$ had Papile grade 3 or 4 IVH at the time of referral. $65 \%$ of the patients were initially managed with therapeutic lumbar punctures. The ventricular indices demonstrated an average post-operative reduction of $7.6 \mathrm{~mm}$. 
Surgical complications occurred in 6 cases (26\%), including 1 decompression haemorrhage, 3 infections and 2 early failures requiring revision. There was no mortality. 20 (87\%) infants underwent conversion to a permanent ventriculoperitoneal shunt, mean 60 days (range 16-326) after the VSGS insertion. 9 infants (39\%) required VP shunt revision within the first year after insertion. Need for early revision correlated with low birthweight and the presence of necrotizing enterocolitis. Conclusion: VSGS is a safe and effective temporizing device in the management of PHH in premature neonates. A high number of patients managed with VSGS requiring permanent CSF shunting which may be related to the high Papile grade of this cohort. Extreme low birthweight and abdominal surgery are risk factors for early permanent shunt dysfunction. Further studies are needed to establish optimal protocols for management of these complex patients.

\section{OP60}

Pre- and post-shunting CSF concentrations of IL-1 $\beta$, Il-6 and TNF- $\alpha$ in young Wistar rats: the role of inflammation in kaolin-induced experimental hydrocephalus

Marcelo Volpon Santos, Luiza Da Silva Lopes, Helio Rubens Machado, Ricardo Santos Oliveira (Rideirao Preto, Brazil)

Introduction: Hydrocephalus is a complex disease whose physiopathology remains unclear. There is an increasing body of evidence stating that inflammation plays a role in the inception and progression of hydrocephalus. This study aimed to evaluate the concentration of inflammatory interleukins IL$1 \beta$, IL- 6 and TNF- $\alpha$ on the cerebrospinal fluid (CSF) of young Wistar rats with kaolin-induced hydrocephalus which underwent an adapted (ventricular-subcutaneous) shunt.

Methods: 23 seven-day-old animals were divided into three subgroups: untreated hydrocephalic $(\mathrm{n}=11)$, shunted hydrocephalic $(n=8)$ and controls $(n=4)$. Hydrocephalus was induced at experimental day 7 through injection of $0.1 \mathrm{ml}$ of a $15 \%$ kaolin solution into the cerebellomedullary cistern. Animals were shunted 7 days after hydrocephalus induction. CSF samples were obtained on experimental day 21 , just before sacrifice, via direct ventricular puncture. The concentrations of IL$1 \beta$, Il- 6 and TNF- $\alpha$ were then measured by enzyme-linked immunosorbent assay (ELISA).

Results: All three interleukins were not detected in the CSF of control rats. Mean values for IL- $1 \beta$ were $62.3 \mathrm{pg} / \mathrm{ml}$ and $249.6 \mathrm{pg} / \mathrm{ml}$ for the shunted and untreated groups, respectively. A mean IL-6 concentration of $104.2 \mathrm{pg} / \mathrm{ml}$ was found in shunted animals, whereas in the untreated group it was 364.7 $\mathrm{pg} / \mathrm{ml}$. Lastly, TNF- $\alpha$ mean values for the shunted and untreated groups were $4.9 \mathrm{pg} / \mathrm{ml}$ and $170.5 \mathrm{pg} / \mathrm{ml}$, respectively. Statistical analysis using Kruskal-Wallis test with a Dunn post-hoc test confirmed that the untreated group had higher levels of these cytokines in comparison with shunted and control groups, which, in their turn, did not differ significantly.
Conclusion: Raised CSF concentrations of IL- $1 \beta$, IL- 6 and $\mathrm{TNF}-\alpha$ are found in kaolin-induced hydrocephalus. Treatment with a ventricular-subcutaneous shunt is effective in reducing such levels. These findings support the presence of inflammatory mechanisms in the onset of this pathology and warrant further studies addressing anti-inflammatory therapy as an option for hydrocephalus management.

\section{OP61}

The GAVCA study - Effect of a mobile health application to assist ventricular catheter guidance in a randomized, prospective multicenter trial

Ulrich W. Thomale, Andreas Schaumann, Georg Bohner, Study Group GAVCA (Berlin, Germany)

Objective: Freehand ventricular catheter placement was reported to be inaccurate. Guidance technologies such as ultrasound imaging, neuronavigation or endoscopy are technically sophisticated relative to the short surgical procedure. The GAVCA study investigates the accuracy of a ventricular catheter guide assisted by a simple mobile health application (mhealth app) in a multicenter randomized controlled simple blinded study.

Methods: In total 139 eligible patients were enrolled in 9 study centers. The optimal catheter placement was evaluated by three components using a grading scale (I-IV), an anatomical position scale of the catheter tip and the rate of primary successful CSF draining puncture. The primary response was defined as primary puncture with grade I ventricular catheter tip position in the ipsilateral ventricle. The rate of intraventricular position of the perforated part of the catheter, the early ventricular catheter failure and the complication rate were analyzed as secondary outcome parameters.

Results: The primary response was reached in $70 \%$ of cases in the guided group versus $56.5 \%$ in the freehand group ( $\mathrm{p}=0.099)$. The rate of incorrect catheter placement (non-primary, grade III+IV placement) was $10 \%$ versus $31.9 \%(\mathrm{p}=0.001)$, respectively. The primary successful puncture rate was $100 \%$ versus $91.3 \%(\mathrm{p}=0.012)$. The catheter perforations location completely inside the ventricle was significantly more often achieved in the guided treatment group (81.4\%) compared to the freehand group $(65.2 \% ; p=0.031)$. No differences were seen in early ventricular catheter failure, complication rate as well as duration of surgery or hospital stay.

Conclusion: The guided application of ventricular catheter enables a simple and safe method of catheter placement. The primary endpoint did show a non-significant difference, while the quality of catheter placement was shown by significantly avoiding incorrect placements and increasing the rate of completely intraventricular location of catheter perforations. Long term follow up is needed in order to evaluate differences in catheter survival in shunted patients. 


\section{OP62}

Clinical and histopathological effects of a ventricularsubcutaneous shunt in kaolin-induced experimental hydrocephalus

Marcelo Volpon Santos, Evelise Oliveira Jardini, Camila Araujo Bernardino Garcia, Thais Helena Romeiro, Luiza Da Silva Lopes, Helio Rubens Machado, Ricardo Santos Oliveira (Ribeirao Preto, Brazil)

Introduction: Shunts are still the commonest treatment option for patients with congenital hydrocephalus. Hypothetically, the deleterious clinical and histological effects of hydrocephalus should be reversed by shunting. This study has aimed to look at potential beneficial effects of an adapted (ventricular-subcutaneous) shunt treatment for young Wistar rats with kaolininduced hydrocephalus.

Methods: 27 seven-day-old animals were divided into three subgroups: untreated hydrocephalic $(\mathrm{n}=12)$, shunted hydrocephalic $(\mathrm{n}=10)$ and controls $(\mathrm{n}=5)$. Hydrocephalus was induced at experimental day 7 through injection of $0.1 \mathrm{ml}$ of a $15 \%$ kaolin solution into the cerebellomedullary cistern. Animals were shunted 7 days after hydrocephalus induction. The presence of hydrocephalus and optimal functioning of the shunt systems was confirmed by pre and post-operative magnetic resonance scans. From a clinical standpoint, differences between groups in daily weight gain and memory performance in the Morris water maze test were analyzed. Reactive astrocytosis and cell proliferation by Ki67 immunolabeling were also evaluated.

Results: Weight gain was similar on both hydrocephalic groups but lower than controls. Shunted animals showed better memory performance than their untreated counterparts. Mean reactive astrocyte count on the corpus callosum and germinal matrix, was 4.8/6.1 for shunted, 2.7/2.5 for the untreated hydrocephalic, and $0.4 / 0.2$ for the control groups, respectively. In addition, Ki67 proliferation index was 2.45 for shunted and 1.36 for untreated animals, whereas control group had an index of 90.5. Using Poisson's double progression model, increased astrocytosis was statistically confirmed in the hydrocephalic groups, as well as a lower number of proliferative cells, as compared to controls $(\mathrm{p}<0.01)$. Also, shunted rats had significantly higher astrocyte counts than non-shunted ones $(p<0.01)$; nonetheless, cell proliferation was not significantly different for these groups $(\mathrm{p}=0.35)$.

Conclusion: The ventricular-subcutaneous shunt for treatment of kaolin-induced hydrocephalus in rats has clinical benefits, but is not sufficient to change the evolution of previously established injury and cellular reparation processes on the hydrocephalic brain.

\section{OP63}

Vancomycin use during CSF-shunt placement reduces shunt infection rate

Martine W.T. van Bilsen, Erik J. van Lindert (Nijmegen, the Netherlands)

Introduction: Postoperative shunt infection is a major contributor of morbidity after shunt implantation. Average cost of a single shunt infection in Europe is estimated at EUR 30.000 to 40.000 . Most shunt infections occur during the first month after implantation and are caused by staphylococci, this implies that a pathogen contamination during surgery is responsible for most postoperative drain infections. To prevent this contamination, multiple measures are suggested in literature of which the latest is an antibiotic impregnated catheter (AIC). The aim of this study was to determine if using a standard shunt immersed in vancomycin $(2 \mathrm{mg} / \mathrm{ml}$ solution), introduced and added to a standard shunt infection protocol (SIP) in April 2012, just before implantation would reduce shunt infection rate (SIR).

Methods: This is a retrospective analysis of all internal CSFshunt implantations and their complications from 2010 onward at the Radboud University Medical Centre Nijmegen. All patients were operated according to a SIP and standard iv cefazoline preoperatively. Cohort 1 consists of all consecutive patients from 2010 and 2011, whereas cohort 2 consists of all patients from April 2012 - April 2015. SIR up to 6 months postoperatively before and after introduction of vancomycin use were compared.

Results: Cohort 1 consisted of 263 patients and cohort 2 of 383 patients of which 131 and 159 respectively were $<17$ years. SIR dropped from $6.8 \%$ to $3.7 \%$ (ns), in adults from $6.1 \%$ to $1.8 \%$ (ns) and in children from $7.6 \%$ to $6.3 \%(\mathrm{~ns})$. SIR per year went from $4.5 \%$ to $5.7 \%$ and $0.7 \%$ after introducing the new protocol

Conclusion: The use of intraoperative vancomycin seems effective in reducing SIR with an effect equal to that reported of AIC. Considering the financial benefits and effectiveness of vancomycin use, further research is needed to compare the value of vancomycin use with AIC use.

\section{OP64}

Principles of testing hydrocephalus shunts in vivo using infusion test

Zofia Czosnyka, Matthew Garnett, Eva Nabbanja, Marek Czosnyka (Cambridge, United Kingdom)

Objective: Hydrocephalus shunts may fail after implantation. Patients may develop adverse symptoms, which are not $100 \%$ specific for shunt failure, with brain imaging not always clear. Our recent study showed that in approximately $50 \%$ of cases referred to Hydrocephalus Clinic for infusion test to check shunt system patency, blockage or overdrainage cannot be 
confirmed. These patients, if revised surgically, would not benefit from surgery.

Methods: Infusion study can be performed via two $25 \mathrm{G}$ butterfly needles inserted into shunt prechamber. Free aspiration of CSF is important marker of ventricular inlet patency. One needle is connected to pressure transducer and second to syringe infusion pump with Hartman solution. After 10 minutes of steady state pressure monitoring, infusion starts with the rate of 1.5 or $1 \mathrm{ml} / \mathrm{min}$. Pressure rises until reaching plateau, then infusion is stopped and pressure decreases towards baseline value. Afterwards patient is sat up (in bed or reclining chair) and pressure is monitored minimum for 10 minutes.

Results: Around 2000 tests were performed in years 19932014 in shunted patients. 200 tests $(10 \%)$ in pediatric patients. Following principles were formulated:

1. Presence of detectable pressure pulse waveform at baseline and through the test is essential marker for patency of ventricular drain.

2. If during the infusion, pressure increases above "critical threshold" established as shunt opening pressure plus shunt's hydrodynamic resistance times infusion rate plus $5 \mathrm{~mm} \mathrm{Hg}$, the valve is judged to underdrain (valve or distal drain obstruction).

3. Normal outflow resistance during infusion test should not exceed $6 \mathrm{~mm} \mathrm{Hg} /(\mathrm{ml} / \mathrm{mi}$ ) (exception: Orbis Sigma Valve). Cases with normal resistance but baseline pressure elevated above shunt operating pressure plus $5 \mathrm{~mm} \mathrm{Hg}$, can result from abnormally elevated abdominal pressure.

4. If during the "tilting test", pressure decreases below $10 \mathrm{~mm} \mathrm{Hg}$ initially and then it further decreases gradually, overdrainage is possible.

Conclusion: Infusion test allows avoiding unnecessary shunt revision.

\section{OP65}

Water on the brain: volumetric assessment of ventricular changes in shunt failure

Ian Anderson, Huw Selley, John Goodden, Gnanamurthy Sivakumar, Atul Tyagi, Paul Chumas (Leeds, United Kingdom)

Introduction: Neuronavigation is well established in tumour surgery. We recently described an innovative use for this software; as a tool to compare ventricular size between serial sets of cranial imaging. We aimed to use this technique to analyse the ventricles of a sample of paediatric patients presenting with ventriculoperitoneal shunt failure.

Methods: The cranial scans for a total of 25 patients were assessed; five each for the standard paediatric age categories (infant, toddler, pre-school, school and adolescent). Patients were selected from an existing shunt database; the five most recent revision shunt operations in each age group were selected.
Ventricular volumes from the last known "well scan" and the immediate pre-op scan were compared using the Smart Brush function of iPlan Cranial version 3.0 software (Brainlab, Feldkirchen, Germany). The radiology reports issued at the time of the scans were also compared.

\section{Results:}

- Measurements suggested that the immediate pre-operative scan showed a relative increase in ventricular volume in 21 cases $(84 \%)$.

- The mean percentage increase in ventricular volume from baseline was $358 \%$ (range $-48 \%$ to $1772 \%$ ).

- 7 patients with proven shunt failure had ventricles that increased by a total volume of $<20 \%$ of baseline.

- 6 patients $(24 \%)$ had demonstrable volumetric change but were reported as "unchanged" by the radiologist. For these patients, the mean percentage increase in ventricular volume from baseline was $57 \%$.

- The mean increase in ventricular size observed in toddlers and pre-school children was less substantial than in other age groups.

Conclusion: Neuronavigation is commonplace in modern neurosurgery. The software allows for quantitative volumetric analysis of any object on a scan (including CSF spaces or tumours). This tool can provide a useful adjunct in comparing subtly different scans as well as for further understanding of what happens to the ventricles at the time of shunt failure in different patient cohorts.

\section{Flash Presentations}

\section{Session 1: Spinal dysraphism}

\section{FP001}

The health related quality of life of children with lumbosacral lipoma

Lindy May, Adam Kucznski, Jo Wray (London, United Kingdom)

Introduction: Lumbosacral lipoma is a rare chronic disease with an unknown natural history. The child may be asymptomatic, have fixed deformities apparent at birth or may develop deficits as the child grows. Chronic illness can affect a child's development as it can change the developmental trajectory and this is particularly relevant during adolescence, when social, psychological and physical issues occur. The Health Related Quality of Life of 54 children with lumbosacral lipoma was assessed with the aim of evaluating changes in morbidity and the efficacy of medical interventions, and to identify the child's individual needs with the aim of improving the patient experience.

Methodology: The Health Related Quality of Life of 54 children with lumbosacral lipoma was assessed using a validated questionnaire (PedsQL) and qualitative interviews. 
Results: Children with lumbosacral lipoma were at risk of a lower Health Related Quality of Life compared to norms, particularly children with transitional lipomas, but many children were within the normal range. Social functioning may be particularly affected and urinary deficits were associated with a reduced Health Related Quality of Life.

Conclusion: Further research is required to identify interventions required including psychology and to evaluate inequalities and plan resources for children with this rare chronic disease.

\section{FP001B}

Congenital neural tube defects evaluation in newborns with prenatal Zika virus infection

Tiago de Paiva Cavalcante, Joana Julia G. de Campos, Marianna Ribeiro de M. Freire, Fabricia Gonzaga Ferreira, Gustavo Henrique Barboza, Tacia Lorena de R. Ferreira, Elizabeth de Freitas M. Teodoro, Rilton Marcus Morias (Aracaju, Brazil)

Introduction: Neural Tube Defects (NTD) occurrence depends of genetic or environmental causes, as ilicit or medical drug use, vitamin insuficiency or gestational infections. Acquired microcephaly is presumed to result from brain injury, like hypoxicischemic injury, intracranial infection or metabolic disease. Both occur in different embryogenesis steps, share similar etiologic factors, sometimes not explained.

Methods: Our prospective series evaluated all newborns up to 5 days of life with or without microcephaly in the high risk Maternity from Sergipe-Brazil, between 01 November and 31 December 2015. Gestational dates about Zika virus mother infection, respiratory tract infections, exanthematic diseases, TORCH assays, ilicit or medical drug use, folic acid supplementation were collected. The children were examined in the search of skin stigmata underlying NTD (dimples, deviated gluteal fold, subcutaneous lipomas, hypertrichosis, hemangiomas and nevus) and other associated systemic malformations. Results: There were examined 33 newborns (10M/20F; gestational age 38,06 $\pm 1,2$ weeks [35-41]) with microcephaly (head circumference $30,34 \pm 2,67 \mathrm{~cm}$ [21-33]) and Zika virus gestational infection associated (13 cases confirmed mother history of clinical or subclinical infecction and 33 negative TORCH assays). No cases presented skin stigmata for NTD, despite brain malformations (ventriculomegaly, calcifications, dysgenesis of corpus callosum, lissencephaly). In the same time 763 newborns without microcephaly were evaluated 16 (8M/9F) presenting skin signs suggesting NTD: dimple (1), deviated gluteal fold (13), hypertrichosis (3).

Conclusions: Zika virus infection belongs to tropical diseases group transmitted by Aedes aegypti mosquito, epidemic in Brazil. First cases of Central Nervous brain congenital malformations related were registered recently. Facing this new disease and lack of knowledge about real cause, absence of skin signs suggesting NTD regardless microcephaly in these children may imply physiophatogy mechanism onset neuronal proliferation and differentiation phases, late for Neural Tube Defects.

\section{FP002}

Postnatal repair of myelomeningocele $-A$ method of the past?

Elke Januschek, Andreas Röhrig, Sandra Kunze, Christian Fremerey, Beatrix Wiebe, Martina Messing-Jünger (Offenbach, Germany; Sankt Augustin, Germany)

Objective: The first attempts of treatment of Myelomeningocele (MMC) were injection of "Morton's Iodo-Glycerine solution" and sac ligation usually with fatal outcome. Later many years postnatal closure of the MMC was the method of choice. Today there are two more approaches to surgical treatment: open and endoscopic fetal surgery.

Methods: In order to compare state of the art MMC repair in Germany with results from MOMS trial and fetoscopic series, a literature review and a retrospective analysis of 49 cases (1/2007-10/2015) with postnatal closure were carried out.

Results: In our series 49 children $(25 \mathrm{~m}, 24 \mathrm{f})$ with spinal dysraphism were included. In 36 children (73\%) Chiari malformation type II was found. $85.7 \%$ suffered from hydrocephalus requiring a shunt. 23 of 33 patients $(70 \%)$ are ambulatory w/wo orthoses or devices. 14 children $<2$ years were assessed separately. The management of myelomeningocele study (MOMS) included 183 patients, 78 of them got open prenatal surgery. In this group 2 perinatal deaths occurred. The maternal complications included inter alia pulmonary edema, oligohydramnios and placental abruption. Shunt implantation rate was $44 \%$, hindbrain herniation rate $65 \%$. Walking w/wo orthoses and devices was possible in $71 \%$. 51 cases with percutaneous fetoscopic surgery are published by the German group. 4 fetal or neonatal deaths are reported, but no information on hydrocephalus or hindbrain herniation. Significant maternal morbidity was seen.

Conclusion: Even though both methods of fetal surgery imply a slightly improved motor function with decrease of hydrocephalus and tonsillar herniation, we could not see significant differences compared to our postnatal MMC repair, e.g. regarding ambulation and motor function. Regarding the fetal, neonatal and maternal risks, postnatal repair is significantly safer with low morbidity and no mortality.

\section{FP003}

Identification of cellular, molecular, and imaging clues for tethered cord development in the context of lipomyelomeningocele 
Gesa Cohrs, Bea Kowitzke, Ivo Leuschner, Michael Synowitz, Janka Held-Feindt, Friederike KnerlichLukoschus (Kiel, Germany)

Introduction: Underlying mechanisms of tethered cord syndrome (TCS) in Lipomyelomeningocele (LMMC) are not well understood. Traction leading to mechanical or ischemic-metabolic spinal cord (SC) injury is considered potential enabling factor. The aim of this study was to investigate further potential cellular processes and molecular mediators, which might promote TCS in the context of LMMC.

Material and Methods: Ethical board approval was obtained. Specimens from 12 LMMC-surgeries harboring neuroepithelial tissue were identified through our neuropathology database. Clinical data (spinal level, neurological status, bowel/bladder dysfunction, development of TCS) were obtained retrospectively. Pre-surgical MRI was reevaluated for lipoma volume, conus position, and potential imaging clues for neural tethering. Controls included normal adult spinal cords $(\mathrm{sc})(\mathrm{n}=4)$. Sections were analyzed semiquantitatively for neuroglial, neural crest, mesenchymal and epithelial marker expression (among others GFAP, neurofilament (NF 200kD), NeuN, synaptophysin, CNPase, Vimentin). Immunohistochemistry and real-time RT-PCR for the inflammatory cytokines Interleukin-1beta (IL-1b), IL-1R1, tumor necrosis factor-alpha (TNF-a) and TNF-R1, was performed. Further, hypoxia inducible factors (HIF-1a/-2a) as indicators for ischemia were investigated. Respective expressions were analyzed qualitatively and semiquantitatively (ImageJ, $1.43 \mathrm{u}, \mathrm{NIH}, \mathrm{USA}$ ). Cellular expression patterns were confirmed via double-fluorescence-immuno-labeling with respective marker antibodies.

Results: All cases exhibited significant induction of GFAP and Vimentin with morphological hints for astrogliosis.IL1R, IL-1b, TNF-R1 and TNF-a Immunoreactivity (IR) were significantly elevated in all cases (significance per case ranging between $\mathrm{p}<0,05$ and $\mathrm{p}<0,001$ vs. control). This IR was co-stained with NeuN, microglial, and astroglial markers. HIF-1a and HIF-2a IR were induced significantly in all cases. Clinical and imaging wise no specific hints regarding TCS development became identifiable.

Conclusion: Astrogliosis was confirmed as potential crucial factor in LMMC placode tissue that might, together with the induction of pro-inflammatory cytokines and ischemic processes promote LMMC associated TCS.

\section{FP004}

\section{Lessons learned after 160 fetal surgeries for myelomeningocele}

Sergio Cavalheiro, Antonio Moron, Italo Suriano, Mauricio Barbosa, Patricia Dastoli, Herbene Milani, Jardel Mendonça,
Stephano Sarmento, Tatiana Sibov, Marcus Devanir, Samuel Salu (Sao Paolo, Brazil)

Introduction: The myelomeningocele (MMC) is a complex malformation of the central nervous system and their hulls. The surgical procedures performed in the fetal period appear to benefit when compared to patients operated on after birth.

Methods: We analyzed clinically and radiologically 160 MMC fetuses intrauterine operated and compared with 160 patients who underwent surgery after birth by the same surgeon and the same medical service. The evolutionary segment ranged from six months to four years. The following aspects were studied: 1) cephalic perimeter; 2) the presence and Chiari degree; 3) posterior fossa volume; 4) clivus-supraocciput angle variation; 5) valve placement; 6) relationship between functional and anatomic level of the lesion.

Results: The operative mortality in fetal surgery was higher than the postnatal, $4.3 \%$ and $1.25 \%$ respectively. All fetuses that died occurred in the first cases operated, and in the last three years, we had more mortality. We did not have maternal mortality. In $84.3 \%$ of postnatal surgical cases require shunts while in pre-natal only $4.3 \%$ needed. The intrauterine operated patients there was a trend macrocephaly associated with nonhypertensive ventriculomegaly. The severity of the Chiari type 2 was lower in intrauterine surgical cases. The volume of the posterior fossa was doubled in patients operated in the fetal period, and the clivus-supraocciput angle was higher in this group of patients. Motor level was better in fetal group than in the postnatal.

Conclusion: Fetal surgery for myelomeningocele correction promotes an increase in the volume of the posterior fossa, turning an obstructive hydrocephalus in a communicating or non-obstructive hydrocephalus with decreasing degrees of Chiari type 2 and improves the motor level of these patients. A learning curve is critical in reducing fetal mortality and a trained multidisciplinary team is important for obtaining good results.

\section{FP005}

Avoiding complications in kyphectomy in myelomeningocele patients: technical changes

Ricardo A. Gepp, Marco R.S. Quiroga, Cicero R. Gomes, Hugo J. Araújo (Brasilia, Brazil)

Introduction: Kyphosis is a serious complication in children with myelomeningocele. This deformity causes skin ulcers, difficulty in positioning and infections. The kyphectomy is an effective surgery for deformity correction, but presents the possibility of surgical complications and postoperative. The authors present some technical changes performed in a number of patients in order to reduce the risk of complications. 
Method: Initially we analyzed the main surgical complications occurred in patients undergoing kyphectomy. The authors identified: infection, skin ulceration due to instrument, failure and breakage of material. Technical changes to decrease the complications were applied. The planning of osteotomy and changing materials positioning were performed to decreased pressure under the skin. Transpedicular screws were applied directly to the vertebral body avoiding pressure on the skin. The paravertebral muscles were used to cover all the instrumentation. The initial series of patients 2006-2011 with 8 patients showed 3 cases of major skin lesions with dehiscence. Between 2011 and 2016 were operated 10 patients with these technical changes and the new series was analyzed and compared.

Results: In the current series there was only one skin lesion in a child. Surgical time remained similar as well as the arthrodesis. There weren't material breaks. The fusion occurred in all patients after six months of fixation. Compared to our previous series there was an improvement of the results. Compared with other results in the literature, we also observed a decrease of surgical complications.

Conclusion: Kyphectomy is an effective surgery with significant reduction in kyphosis. The skin lesions are a major complication and can cause serious infections. Observance of surgical care can reduce the risk of this complication.

\section{FP006}

Nurse led care of MDT clinic for children with spina bifida Lindy May, Lucy Alderson (London, United Kingdom)

Nurse led care of MDT clinic for children with spinabifida was introduced to free up neurosurgical consultant time to see new neurosurgical referrals. In addition, education alongside assessment was recognized as key for management of these children and as such, the primary assessor need not be a neurosurgeon. The clinic now comprises a nurse consultant in pediatric neurosurgery, a senior neuro physiotherapist and a urology nurse specialist.

\section{Methodology:}

- A review of the literature was undertaken to identify differences in nurse led and clinician led clinics.

- An assessment of the needs of the children attending the spina bifida clinic was undertaken.

- Discussions took place with the neurosurgeon to ensure good communication channels were established to troubleshoot any queries promptly.

\section{Results:}

- The BMJ published a Meta analysis of 34 clinical studies which identified greater patient satisfaction with nurse led care than physician and that there were no significant differences in health outcomes. The review also identified improved communication and information in nurse led clinics, than those led by physicians.

- The needs of the children attending the clinic were identified and included issues relating to neurosurgery (hydrocephalus, Chiari, spinal cord retethering), mobility and orthopedic issues, urology and bowel management, psychology, transition to adult services, communication with local services and child/parent education.

- Clear communication channels were established between professionals and "red flags" identified.

\section{Conclusion:}

- The MDT clinic has been running effectively with parents and children reporting satisfaction with the clinic set up.

- Communication with the neurosurgeon has been effective (eg. discussion of new symptomology related to shunt blockage, Chiari or cord retethering).

\section{FP007}

Role of anticholinergics and gabapentin in management of neurogenic bladder after repair of spina bifida - A randomized controlled study

Monika Bawa, Vedarth Dash, Jai K. Mahajan, K.L.N. Rao (Chandigarh, India)

Introduction and Aims: The role of anticholinergics in the management of neurogenic bladder is well established however some patients have sub optimal response or severe side effects to the standard anticholinergic therapy. This study is designed to assess and compare the efficacy of Gabapentin with oxybutynin in the management of neurogenic bladder associated with lumbosacral myelomeningoceles.

Methods: Patients operated for LSMMC who met the inclusion criteria were subjected to base line investigations including urodynamic studies. They were then randomized into three groups and started on oxybutynin, gabapentin and combination of both respectively. Clean intermittent catheterization was started in all these patients. A thorough clinical and urodynamic reassessment was done at six months and one year.

Results: Forty four patients ranging from 3 years to 19 years (median 5 years) were included in the study. Improvement was noted in all the patients in both the symptomatic as well as the urodynamic parameters in all the groups. Maximal improvement of symptom score was seen in patients with combination of the drugs at 1 year (mean $=6.1$ ). In the urodynamic studies the compliance, bladder pressures and the bladder capacity showed improvement. The difference was significant between the groups at both six months and 1 year for bladder pressures and volume. The improvement in the compliance though marked was not statistically significant. The best response was seen in the patients receiving both the drugs rather than any one. Gabapentin was found to be better tolerated than oxybutynin. 
Conclusion: Gabapentin is a good alternative to oxybutynin for the management of patients of neurogenic bladder sphincter disorder both as monotherapy and as an add-on therapy. It has potential application in patients of detrusor hyperreflexia with inadequate response with anticholinergics as well as initial therapy in patients not tolerating or compliant with the standard therapy.

\section{FP008}

The neurenteric canal: why it is important to revisit embryology before ascribing malformations to normal structures?

Martin Catala, Michel Zerah (Paris, France)

Many malformations of the spinal cord are classified under the generic term neurenteric malformations or split notochord syndrome. They are considered as due to the persistence of a normal channel called the neurenteric canal. This structure which connects the lumen of the neural tube with that of the primitive gut was initially described by Alexander Kowalevsky in the tunicate. Kowalevsky in his work published in 1871 already attributed to the canal the meaning of a persistent blastopore (Einstülpungsöffnung). Then, such a channel is evidenced in amphioxus, fish, amphibians and reptiles. It has never been observed in birds. In mammals, this structure is not described up to the description of a human embryo in 1889 by Ferdinand Graf von Spee of an opening he considered as equivalent to neurenteric canal. Since this observation such a structure is regularly demonstrated in human embryos. It is interesting to note that it is never observed in other mammals whose preservation conditions are much better. Ronald O'Rahilly and Fabiola Müller in 1981 show frequent artefacts in all human embryos previously reported. They wrote that the neurenteric canal is almost never visible in human embryos that have been fixed correctly and concluded that this channel is virtual. Phylogenetic analysis of mammals strongly suggests that neurenteric channel does not exist in humans and this should be checked on early embryos correctly preserved. Under these conditions, it appears necessary to review the assumptions accounting for the emergence of such defects. To do this, we must remember that the notochord is not formed under the same mechanism in the anterior spinal region and in the posterior one. Indeed, notochordal presumptive cells are inserted directly in the endoderm in the anterior region. Then, they undergo a movement of excalation which is disrupted in mice in which Noggin has been invalidated. In contrast, in the posterior region of the future spinal cord, the notochord is formed immediately as a layer independent from the endoderm. These data should lead to reconsider the cases of spinal neurenteric cysts to determine whether the anterior and posterior forms differ.

\section{FP009}

Philosophy for management of split cord malformation Ashok Kumar Mahapatra (Bhubaneswar, India)

Split Cord Malformation (SCM) is a rare condition and only few large series are published in the world literature. Over 25 years, we have operated more than 400 cases of SCM of various types. In 2004-05, we sub classified $\mathrm{SCM}$ to $\mathrm{A}, \mathrm{B}, \mathrm{C}$ and $\mathrm{D}$ categories depending on the length of the split cord and position of the spur and space available below and surroundings of the spur. We also operated asymptomatic patients as the risk of deterioration is less than $5 \%$. In 25 years, we have operated 35 patients' asymptomatic cases. Improvement was recorded $55 \%$ and stabilization $41 \%$ and in $4 \%$ cases there was deterioration. Amongst those deteriorated, the maximum deterioration was observed in $1 \mathrm{D}$ Sub type.

Conclusion: In our experience, all SCM patients must be operated even the patient is asymptomatic. The chance of deterioration is less than $5 \%$, hence it is better to take the risk and do the best, as we know once deteriorate improvement chance is only $50 \%$.

\section{FP010}

Surgical treatment of congenital split cord malformation Ai-Jia Shang (Beijing, China)

Objective: To study the surgical treatments of congenital split cord malformations (SCM).

Methods: The clinical data of 65 in-patients with split cord malformation from January 2008 to January 2013 were analyzed retrospectively. Among 65 patients, there were $37 \mathrm{fe}-$ males and 28 males, aged 3 to 46 , with an average age of 17.2. Age distribution was that 17 cases aged 3 to 12,27 cases aged 12 to18, 21 cases aged over 18. According to Pang's SCM classification, the patients can be classified as SCM-I and SCM-II: SCM-I are cases with osseous septum and two spinal cords located in two different duramater capsules respectively, and SCM-II are cases with membranous septum and two spinal cords located in one duramater capsule. Within these 65 cases, 41 were diagnosed with SCM-I, and 24 were diagnosed with SCM-II. Longitudinal fissure was located in thoracic, thoracolumbar, lumbar, and lumbosacral segments of 19, 18, 23 and 5 cases, respectively. Surgical treatments were performed in all the 41 cases with SCM-I and 18 of 24 cases with SCM-II. Symptoms of 6 adult cases with SCM-II were stabilized and under observation. Different surgical methods were used for cases with SCM-I and SCM-II. For SCM-I, the key points of the surgeries are the excision of osseous septum of longitudinal fissure and simultaneous release of the pressure on spinal cords from dura, especially at the caudal part of 
longitutinal fissure. During the procedure, part of dura was excised and sewed up. Simultaneously, the dura mater cyst was reshaped and the filum terminale was excised. For SCM-II, the cause of fiber bundles should be analyzed. Fibrous septums and belts should be excised for cases with them at longitudinal fissure. For the cases with no fibrous septums and belts, filum terminale should be excised.

Results: Among the 59 cases undergone surgery, 50 were followed up for 3 to 48 months (with an average of 16 months). According to the sensation and movements in the lower extremities and excretory functions, the conditions of patient undergone surgeries will be evaluated by the Roy scoring method. Respectively, there are 15 significant cases, 25 partially improved cases, 25 stabilized cases and 1 exacerbated case. No exacerbation of symptoms was observed and the condition was stabilized in 6 cases with SCM-II undergone no surgery, after being followed up for 6 to 60 months.

Conclusion: According to different types of SCM, different surgical methods should be applied in surgical treatment. Early surgery should be considered for all cases of SCM-I, including the asymptomatic cases. The key points of the surgery are release of the pressure on spinal cords from osseous septum and simultaneous excision of the filum terminale. For SCM-II, the method of surgeries should be chosen according to the cause of tethered spinal cord. For the cases with definite separation and fibrous belt stretch diagnosed by imaging examination, excision of septum and release of fibrous belt stretch should be performed in the surgery. For the cases with SCM-II and no pressure on spinal cord from fibrous septum excision of filum terminale should be selected.

\section{FP011}

\section{Split cord malformation: clinical experience}

Mehmet Can Ezgu, Cahit Kural, Mehmet Ilker Ozer, Ozkan Tehli, Yusuf Izci (Ankara, Turkey)

Introduction: Split cord malformation (SCM) is one of the most difficult subjects of pediatric neurosurgery and a rare congenital anomaly in which the cord is split over a portion of its length to form double dural tubes (Type I) or two hemicords in a single dural sheath (Type II). Patients may be asymptomatic or severely affected. Our aim is to present clinical features of 10 cases with spinal split cord malformation treated in our instution between January 2010 and October 2015.

Method: All of the patients were treated due to SCM between January 2010 and October 2015 at the Gulhane Military Medical Academy. All of the patients were operated with assistance of intraoperative neuro-monitoring system. Data for each patient were evaluated retrospectively, and age, sex, clinical findings, localization of the malformation, surgical results and histopathological findings were recorded.
Result: There were 3 females and 7 males patients. Their ages range between 0 and 13 years. $7(70 \%)$ of the cases had lumbar and $3(30 \%)$ of the cases had thoracic SCM. None of the patients had cervical SCM. $4(40 \%)$ of the patients had meningomyelocele which is the most common reason to seek medical care. $9(90 \%)$ of the patients had a neurological deficit at initial evaluation. $5(50 \%)$ of the patients had their split cord malformation localized at second lumbar vertebrae region. The most common histopathological findings were fibroadipose, fibroosseous and degenerated neural tissue. There was not neurological or neuromonitoring worsening at postoperative period.

Conclusion: All patients who are diagnosed with SCM should be operated to prevent further neuro-urological deterioration. Using intraoperative neuromonitoring is recommended for SCM surgeries.

\section{FP012}

Stem cells from different fetal tissues: isolation and characterization by comparative study of expression of some pluripotent markers

Tatiana Sibov, Lorena Pavon, Luciana Marti, Daniela Oliveira, Antonio Moron, Sergio Cavalheiro (Sao Paolo, Brazil)

Introduction: The amniotic fluid and the amniotic membrane are sources of different populations of stem cells. Although much of the amniotic fluid and membrane share a common embryonic origin, the specific origins of the stem cells found in these two sources remain to be determined. Nevertheless, pluripotent cells subpopulations has been described in these stem cells sources. Here, we study and characterized the immunophenotypic profile of some pluripotency markers in stem cells from amniotic fluid and amniotic membrane, thinking later the most important clinical/preclinical applications.

Methods: The samples were collected, cultured and analyzed for the cell-surface expression with a pre-defined set of protein markers related to pluripotency by flow cytometry, and differentiated in osteogenic cells.

Results: FACS analysis showed that stem cells from these sources were positive for the typical mesenchymal markers, low or no expression of MHC class I antigens, HLA-DR and hematopoietic cells markers, and absence of MHC class II antigens. These cells also expressed the homing markers CXCR4 and CCR2, and embryonic stem cells pluripotency markers, like Tra-1-60, Stage Specific Embryonic Antigen 4 (SSEA-4), Sox2, Nanog and Octamer Binding Transcription factor $3 / 4$ (Oct3/4). Expression of SSEA-4, Tra-1-60 and Sox 2 was higher in the samples of amniotic fluid than in other samples.

Conclusion: Thus, the expression of these markers suggest that these stem cells populations have important therapeutic characteristics as plasticity, reduced immunogenicity, 
anti-inflammatory potential and the ability to migrate to a site of tissue injury and inflammation, as well as, a crucial role in the maintenance of pluripotency and self-renewal.

\section{Session 2: Advances in pediatric head trauma management}

\section{FP013}

Prognostic value of leucocytosis in pediatric traumatic brain injury

Soumya Mukherjee, Bethanabatla Ramakrishna, Gnanamurthy Sivakumar, John Goodden, Atul Tyagi, Paul Chumas (Leeds, United Kingdom)

Introduction: Head trauma in adults has been correlated with leucocytosis. Proposed mechanisms include acute phase response-mediated rise in catecholamine and cortisol levels, and inflammatory microglial activation from brain oedema formation. In a pilot study we assessed leucocytosis and its prognostic value in paediatric traumatic brain injury (TBI).

Methods: Twenty consecutive paediatric patients with isolated severe, moderate and minor TBI were retrospectively analysed. Data collected included initial leucocyte count (WCC), presenting Glasgow Coma Score (GCS), computed tomographic (CT) findings, hospital stay, and Glasgow Outcome Score (GOS). The relationship between these parameters and WCC were statistically evaluated.

Results: Mean age was 2.2 years (range, $0.2-12$ years) with a male-to-female ratio of $3: 1$. Seven, six and seven patients had severe, moderate and mild TBI, respectively. For patients with GCS 3-8, 9-13 and 14-15, WCC was $20,15.1$ and $10.7 \times 10^{9} / \mathrm{L}$ and neutrophil counts were $14.6,11.3$ and $6.1 \times 10^{9} / \mathrm{L}$, respectively. Differences in WCC and neutrophil count were significant between the different GCS groups $(p<0.01)$. WCC and neutrophil count were greater in patients with significantly abnormal CT findings including basal cistern effacement and/or midline shift $>5 \mathrm{~mm}$ as compared to patients without these imaging features $(p<0.05)$. Length of hospital stay correlated with WCC and neutrophil count $(\mathrm{p}<0.05)$. Furthermore, the GOS value increased as the WCC decreased and this relationship was significant $(\mathrm{p}<0.05)$.

Conclusion: Our pilot study has shown that high leucocyte count and neutrophil count have a predictive value for poor GCS, severe CT findings, lengthy hospital stay and poor GOS in isolated paediatric TBI. Complete blood cell count should be included in the initial laboratory work-up for these patients as it may represent an easily obtainable and potentially important prognosticator of clinical course and outcome. Future prospective study in a larger cohort is planned to verify our findings.

\section{FP014}

Perforating brain injuries in children - Protocol of management from civilian practice

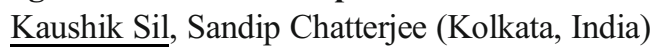

Introduction: Perforating injuries to the brain are caused by a number of blunt and sharp objects in civilian life. This is a report of 15 children with perforating brain injuries which presented to our Centre from 2007 and 2014.

Methods: The average age was 5.7 year and 9 were male and 6 female. The etiological agent ranged from spookes of bicycle wheels to idli makers to sharp end of umbrellas. After emergency resuscitation, and a detailed history from witnesses, the radiological investigation useful in all cases was MR scan of brain. Important findings include: entry and exit sites; intracranial fragments; missile track and its relationship to both blood vessels and air-containing skull-base structures; intracranial air; transventricular injury; basal ganglia and brain stem injury; missile track crossing the midline; multi-lobar injury; basal cisterns effacement; brain parenchymal herniation and associated mass effect. In 6 of the cases, the missile tract was suspected to be crossing vascular territory and hence angiography was resorted to. In 3 cases, pseudoaneurysm or arteriovenous fistulae were detected. Although intracranial pressure monitoring was used by us when indicated, in only 1 of the 15 cases did we feel it necessary to measure this parameter.

Although debridement is recommended in al cases, we did not intervene in 4 cases where the tract penetrated to deep levels in eloquent areasand where the children arrived very late. In one case the child came with a delayed abscess which required drainage.

Results: Bad prognosis in our small series occurred in bilateral hemisphere injuries, in children presenting with low Glassgow Coma scores or with respiratory distress.

Conlusions: We would like to propose a algorithm for management of these uncommon injuries in the pediatric age group.

\section{FP015}

Cranial burst fracture in infants - An uncommon injury Sandip Chatterjee (Kolkata, India)

Introduction: Cranial burst fracture, typically associated with severe injury in infants less than 1 year of age, is a closed, widely diastatic skull fracture accompanied by acute cerebral extrusion outside the calvarium. An uncommon injury, it needs to be recognized early for appropriate treatment.

Methods: We report a series of 12 children with cranial burst fractures treated by us from 2005 to 2014 with a minimum 1 year followup. 
There were 8 males and 4 females, with age range between 3 months and 1.8 years, with 3 being children above the age of 1 year. Presentations included infants with suspected scalp haematomas who went into status epilepticus or developed major neurodeficits (8 cases), or babies that clinically deteriorated more rapidly than suggested by their CTScans (4 cases).

MRScan was done in all cases, CTScan was done in 5 of the infants. After stabilization, emergency surgery was performed in the shape of excision of herniated cerebral tissue, duroplasty and cranioplasty. Cranioplasty was performed immediately in 7 cases, and delayed in 5 cases due to significant brain swelling.

Results: Of the 12 babies, 8 had marked neurdeficit at the time of presentation, and 3 of these had permanent deficits even after surgery with documented venous infarcts on MRScans. The remaining 4 had no neurodeficit at 1 year followup. Cranioplasty was performed with split thickness calvarial grafts in 9cases, and with artificial bone cement in the remaining 3 .

Conclusion: Early recognition of this unusual condition is necessary for quick surgical intervention which prevents retrograde venous thrombosis and leads to permanent neurodeficits. Any infant with a scalp "haematoma" but neurodeficit which is out of proportion should have emergency MRscan and early intervention.

\section{FP016}

Decompressive craniectomy for severe traumatic brain injury in pediatric patients

Kenichi Usami, Juliette Montmayeur, Thomas Blauwblomme, Kevin Beccaria, Giovanna Paternoster, Timothe de Saint Denis, Philippe Meyer, Christian SainteRose, Michel Zerah, Stephanie Puget (Paris, France)

Introduction: Decompressive craniectomy (DC) is performed to decrease the raised intracranial pressure as a second-line treatment for severe traumatic brain injury (sTBI). The definitive indication and the timing of DC for sTBI in pediatric patients are still unclear. Aims of this study are to clarify the clinical characteristics of pediatric patients who underwent DC for sTBI and to find prognostic factors.

Methods: Thirty-three children who underwent DC for sTBI between 1999 and 2015 were retrospectively reviewed. Patients in whom the worst GCS score was more than 8 were excluded. Mean age at injury was $8.1 \pm 4$.1 years. Patients were divided into two groups based on Glasgow Outcome Scale (GOS) score, poor outcome (GOS score 1 - 3, n=18) and favorable outcome (GOS score 4 and $5, \mathrm{n}=15$ ).

Results: Significant differences between poor and favorable outcome was found in the initial GCS score evaluated by ambulance stuffs $(5.1 \pm 2.2$ vs $7.0 \pm 2.4, \mathrm{p}=0.047)$, and in the delay from injury to $\mathrm{DC}(32.5 \pm 47.9$ vs $9.5 \pm 10.8$ hours, $\mathrm{p}=0.04$ ). Age at injury, mechanism of injury, clinical symptoms at initial evaluation, any neuroradiological findings, and the value of intracranial pressure (ICP) did not affect outcome. Conclusion: Earlier DC achieves favorable outcome regardless of the value of ICP. In case of sTBI in padiatric patients with intracranial mass lesion, DC should be performed as soon as possible irrespective of the age, type of lesion, or type of injury.

\section{FP017}

Transcranial ultrasonography in pediatric patient with traumatic brain injury

Mikle Talabaev, Evgenij Mironetc, Kevin Fernando Venegas Hidalgo (Minsk, Belarus)

Introduction: To describe the possibility and efficacy of transcranial ultrasonography (TUS) in pediatric patient of first year of life with traumatic brain injury (TBI).

Methods: 341 patients with moderate traumatic brain injury (TBI II) and severe (TBI III) were admitted in the pediatric neurosurgery department of the Republican Research and Clinical Center of Neurology and Neurosurgery in the period from 2013 to $2015.94 .73 \%$ (323 patients) of them had TBI II and $5.27 \%$ (18 patients) - TBI III.

Results: 224 (65.7\%) patients were under the age of first year of life. According the mechanism of injury, fall down were in $90 \%$ of the cases, child abuse occurred in 3.1\% (7). Linear skull fractures were revealed in $83 \%$ (186) patients, depressed fractures in $2.7 \%$ (6) cases, by x-ray imaging were identified the fracture only 114 (61.3\%) and the rest was not diagnosed. All patients we performed TUS in order to exclude intracranial pathology, and the result in 20 patients was found epidural hematoma, 6 patients was found a significant epidural hematoma which was needed emergency surgery, as 5 patients with subacute subdural hematoma. All children with suspected abnormalities on TUS and worsening neurological symptoms had CT scan either.

Conclusions: TUS is a reliable screening method that allows evaluating the dynamics and changes in younger patient with TBI, beside CT scan for diagnosis of TBI that exposes to radiation to the child.

\section{Session 3: Spine}

\section{FP018}

Spinal kyphotic deformity after laminoplasty in children Christos Chamilos, Spyros Sgouros (Athens, Greece)

Objective: Laminoplasty has emerged in recent years as deformity preserving alternative to laminotomy. The purpose of this study is to analyze the short-to-medium-term kyphotic deformity incidence in a small series of pediatric patients. 
Materials and Methods: We analyzed retrospectively 10 patients operated for various pathologies (tumor, cysts, vascular) with laminoplasty in the last 7 years in a single institution. The laminoplasty was performed with craniotome and the laminas were attached back with sutures (cranially, caudally and on both sides laterally). We reviewed the incidence of subsequent kyphosis along with other factors (age, sex, pre-op deformity, levels of laminoplasty and pathology type). We measured kyphosis with the Cobb angle in the sagittal plane.

Results: The mean age was 9.8 years (range: $7 \mathrm{~m}-18 \mathrm{y}$ ) and the mean duration of follow-up was 3.3 years (range 1-7.5 y). There were 6 tumors, 2 cysts (arachnoid and dermoid) and 2 AVM. Extent of laminoplasty was: 5 levels in 3 patients, 4 levels in 3 patients and 3 levels in 4 patients. The level laminoplasties were: 2 thoracolumbar, 3 thoracic, 1 cervical, 2 cervicothoracic and 2 lumbosacral. At latest follow up, only 2 patients $(20 \%)$ developed mild kyphosis (1 irradiated for spinal cord glioblastoma, Kobb: $32^{\circ}$ and 1 with vascular epidural malformation and adult body type, Kobb: $40^{\circ}$ ).

Conclusion: In our series there was no significant deformity after laminoplasty in the short to medium term follow up. There was no obvious correlation with the underlying pathology. The technique of laminoplasty appears safe with respect to subsequent risk of kyphosis. Longer follow-up is needed in order to determine the long-term deformity risk.

\section{FP019}

Surgical treatment and curative effect analysis in 326 children with tethered cord syndrome

Ai-Jia Shang (Beijing, China)

We explore surgical treatment and curative effect analysis in children with tethered cord syndrome (TCS). According to the clinical manifestations and imaging findings, a total of 326 cases of children with TCS were classified into five types, namely tight filum terminale type, myelomeningocele type, lipomatous malformation type, postoperative adhesions type and split cord malformation. All these cases were taken microscopic surgery. All Children were followed up for $3 \sim 36$ months with an average of 15 months. The curative effects were analyzed by Spina Bifida Neurological Scale (SBNS) which is based on sensory and motor function, reflexes, bladder and bowel function. The result indicates that the total effective rate of postoperative children with TCS was $74 \%$. Different types of efficiency were as follows: tight filum terminale type $90 \%$, myelomeningocele type $84 \%$, lipomatous malformation type $65 \%$, postoperative adhesions type $75 \%$ and split cord malformation $82 \%$, respectively. Early diagnosis and microsurgical operation are keys to the treatment of TCS. The operation effect and prognosis are mainly related to TCS types, operation timing, and severity of symptoms, neural neurolysis and retethering. Suitable clinical classification for TCS is helpful in deciding the prognosis and guiding the treatment.

\section{FP020}

Cerebellar tonsil resection for treatment of Chiari I malformation in children

$\underline{\text { Bruno Braga, }}$ Dale Swift (Dallas TX, USA)

Introduction: Surgical treatment of Chiari I continues to have a relatively high recurrence rate. Although more invasive, resection of the cerebellar tonsils may improve outcome in selected cases. Methods: We reviewed 137 children surgically treated for Chiari I Malformations between 1995 and 2014. Surgical indications were the presence of clinically relevant symptoms or an expansile syrinx. Procedures were classified into 3 different groups: foramen magnum decompression by bone removal and duraplasty with or without arachnoid dissection only (SOC), decompression with bilateral coagulation of tonsils $(\mathrm{SOC}+\mathrm{TC})$, and decompression with subpial resection of the tonsils ( $\mathrm{SOC}+\mathrm{TR})$. The choice for a procedure was based on anatomic characteristics and surgeon's preference.

Results: Mean age was $7.5 \pm 5.4$ years. Fifty-four patients had syringomyelia upon presentation (39.4\%). SOC was performed in 26 patients (18.9\%), SOTC in $24(17.5 \%)$, and SOTR in $87(63.5 \%)$. Mean follow up was $31.4 \pm 34.2$ months and it ranged from 6 months to 12 years. Improvement in symptoms was not statistically different between the 3 groups. Efficacy was also measured as syrinx outcome: improvement in $90.3 \%$ of the patients that underwent SOC + TR ( 28 out of $31), 90 \%$ in SOC + TC (9/10) and $62.5 \%(9 / 13)$ in SOC. There was no mortality. Complications occurred in a total of 10 patients $(7.3 \%)$; there were 4 complications from SOC (15.4\%), 2 from SOTC (8.3\%) and 4 from SOTR (4.6\%). There was no vascular or neural injury secondary to tonsillar resection. Five (3.6\%) patients were reoperated because of persistent symptoms or syrinx, and the rate of reoperation was not statiscally different between the groups.

Conclusion: Surgical treatment of Chiari I malformation in children with SOC+TR provides excellent long term decompression of the cervicomedullary junction without an increase in surgical morbidity.

\section{FP021}

Minimally invasive spine surgery techniques in children Michal Tichy, Jiri Steindler, Robert Lesko (Prague, Czech Republic)

Introduction: Less than one percent of the total quantity of all surgical interventions for lumbar disc herniation is done on patients between the ages of 11 to 20 years old. Back pains occuring to population under 11 years old are much more 
likely to be a manifestation of other diseases (tumours, trauma, congenital anomalies, inflammations). Using the considerate of minimally invasive spine surgery (MISS) techniques enables faster recovery to normal patient's life activity.

Method: We use MISS techniques for child and adult patients at our clinic from the year 2004. Microdiscectomy falls into our most frequently applied type of surgery. It is done using METRx system (Minimal Exposure Tubular Retractor). Due to low incidence of child patients with this particular diagnosis we have accomplished so far 16 clearly miniinvasive operations.

Results: The average age of our patients has been 16 years (span from 14 to 19), the sex ratio is 12 males: 4 females. We did not record neither peroperative nor postoperative complications, none of these patients was necessary to reoperate (the follow-up spans from 1 to 9 years, average 4,5 years), practically all operated patients are completely satisfied without any complications in ordinary life activities

Conclusion: Minimally invasive spine surgery in young patients is according to our experience more suitable than previous more extensive methods. We believe that qualitative and quantitative development of these modern techniques in spinal surgery will continue in coming years.

MISS techniques in young patients improve the long time results and decrease the postoperative consequences.

\section{FP022}

Urodynamic outcomes of detethering in children: experience with 46 pediatric patients

Murat Geyik, Surma Geyik, Haluk Șen, Serhat Pusat, Mehmet Alptekin, Ali Erdem Y1lmaz, Mert Nazik, İbrahim Erkutlu, Yusuf Izci (Gaziantep, Turkey; Ankara, Turkey)

Background: Tethered cord syndrome (TCS) manifests with neurological and urological complaints. Surgical detethering is the main treatment for TCS. Children with urological symptoms are required objective pre- and postoperative evaluations in order to predict urological outcome. The aims of this study are to investigate the effects of detethering procedure on the urodynamic tests and to determine the relationship between the changes of urodynamic tests and the patients's age and gender.

Material and Methods: The data of urodynamic tests in 46 pediatric patients, who underwent surgery for TCS, were retrospectively evaluated. Bladder capacity, postvoiding residual urine volume, maximum intravesical pressure and bladder compliance of each patient were measured in preoperative period and at the 3rd month after surgery. These parameters were statistically compared and the correlations of these parameters with age, gender and etiology of the TCS were also investigated.

Results: Bladder capacity and post-voiding residual urine volume were decreased and maximum intravesical pressure and bladder compliance were increased after surgery for TCS. The decrease in bladder capacity was significant in patients older than 10 years. The increase in maximum intravesical pressure was also positively correlated with age. But the increase in bladder compliance was negatively correlated with age.

Conclusion: Bladder capacity, post-voiding residual urine volume and bladder compliance are mainly affected by surgery in patients with TCS. From the urological viewpoint, children older than 10 years are most likely to benefit from surgery for TCS.

\section{Session 4: Pediatric Neurosurgery and Technology}

\section{FP023}

Intraoperative magnetic resonance imaging in pediatric neurosurgery: safety and utility

Mario Giordano, Amir Samii, Anna Lawson Mclean, Helmuth Bertalanffy, Rudolf Fahlbusch, Madjid Samii, Concezio Di Rocco (Hannover, Germany)

Introduction: The use of high field intraoperative magnetic resonance imaging (iMRI) utility has been largely studied for the treatment of intracranial tumors in adult patients. In this report we investigate the safety, advantages and limitations of high field iMRI for cranial neurosurgical procedures in children with particular attention to craniopharyngiomas and gliomas.

Methods: We performed 82 surgical procedures in children under 16 years of age (range $0.8-15$ years) over an 8 year period (2007-2014) using iMRI. The population was divided in 3 groups: sellar region tumors (group 1), gliomas (group 2), and other pathological entities (group 3). Preand postoperative neurological status, presence of residual tumor, number of intraoperative scans and complications were evaluated.

Results: In group 1 gross total removal (GTR) was performed in $22(88 \%)$ and subtotal removal (STR) in $3(12 \%)$ cases. In group 2 GTR, STR and partial removal (PR) were obtained respectively in $15(56 \%), 7(26 \%)$ and $5(18 \%)$ procedures. In group 3 a GTR was carried out in $28(93 \%)$ and STR in $2(7 \%)$ patients. In craniopharyngioma (group 1) and glioma (group 2) cases where a complete removal was planned the iMRI control allowed to localize residual lesion and reach the surgical goal through further resection respectively in $18 \%$ and $27 \%$ of the procedures. Moreover, in gliomas the resection could be extended from partial to subtotal in $50 \%$ of the cases. In $17 \%$ of the patients belonging to group 3 iMRI enabled the identification and further removal of tumor remnants. There was no intra- or postoperative complication related to the use of iMRI despite special technical difficulties in smaller children.

Conclusions: In our study the use of iMRI in children proved to be safe. The iMRI was most effective in increasing the tumor's extent of resection especially in patients affected by 
low-grade gliomas and cranipharyngiomas. The most prominent disadvantage of high-field iMRI was the limitation in terms of operative positioning due to the configuration of the surgical table.

\section{FP024}

\section{Medico-legal issues and claims that may arise following fetal surgery}

Wan Tew Seow (Singapore, Singapore)

In the past, fetal surgery was performed on fetuses whose conditions were life-threatening.The recent MOMS study on the antenatal closure of open neural tube defects in foetuses, has shown that fetal surgery is feasible to improve the health of infants with a non-life-threatening disorder. This is despite the risks to both mother and foetus. With the slow but increasing numbers of fetal surgeries being carried out to treat such conditions, the possibility of medico-legal claims agains neurosurgeons performing fetal surgery which resulted in severe complications and fetal death is likely, although such claims are likely to be complicated by the legal status of the foetus as a nonperson ("en entre sa mère") in countries where abortions are legalised.

Although no such claims in common law have been reported (to our knowledge), it is worthwhile to know and understand what type of claims the fetal surgeon may face in the future. This can be done by studying past claims on fetal death in criminal and tort cases (such as medical negligence) and such claims include wrongful death actions, maternal consent issues, failure to inform with regards to possible antenatal surgery, decision making in multiple gestation cases, wrongful birth actions and even the speculative wrongful life actions. Understanding such claims can in turn help the fetal surgeon reduce his/her risk of medico-legal claims should a serious adverse outcome occur following fetal surgery.

\section{FP025}

Electronic patient-doctor communication: exploring needs and requirements of pediatric neurosurgeons and patients Nina Klein Essink, Martine van Bilsen, Erik van Lindert, Dutch Pediatric Neurosurgery Society (Huissen, the Netherlands)

Introduction: In the Netherlands, centralization of pediatric neurosurgery is taking place in order to improve standards of care. However, such physical centralization can bring unavailability of care and expertise in other centers and increase travelling time for patients. To overcome these physical boundaries, modern communication technologies can be used to create a virtual pediatric neurosurgical centre. The goal of this study was to investigate support for new means of communication in both pediatric neurosurgeons (PN) and patients and identify possible barriers in order to create a feasible digital platform.

Methods: Semi-structured interviews regarding several means of communication were held with parents, patients, and PN.

Results: Nine out of fourteen Dutch PN were interviewed, covering all pediatric neurosurgical centers. Twelve interviews were held with parents of which two involved children. Saturation of information was reached. Half of PN regularly have telephonic consultations. Most patients would like more telephonic consultations, however they emphasize that clinical evaluation should be possible at all times. Opinions differ on video consultations: addition of non-verbal communication is considered a pro, but there is a lack of trust in technology and planning. Half of PN fear an overload of emails by giving patients the possibility to email them, although PN already using email do not experience this. An intermediate, e.g. a nurse practitioner, to prioritize and answer emails if possible is desired by most. Most patients and PN would like a central website to improve information flow and reduce consultation time. Mentioned disadvantages are superfluous information and costs. Surgeons are willing to collaborate in creating a national website.

Conclusion: Most patients are ready for virtual communication, but surgeons are more hesitant. An intermediate could help overcome mentioned barriers. A national pediatric neurosurgery website can be developed to provide clear information and shorten and/or reduce the number of physical consultations.

\section{FP026}

Motor plasticity in the human cerebellum: an electrophysiological and behavioral study

Pierre-Aurélien Beuriat, Carmine Mottolese, Nathalie Richard, Alexandru Szathmari, Federico Di Rocco, Blandine Grassiot, Angela Sirigu, Michel Desmurget (Lyon, France)

The role of the cerebellum in sensorimotor control and learning is now clearly demonstrated. However, clinical observations show that large cerebellar lesions lead to no or little motor deficit. It can be suggested that there are compensatory mechanisms. In support of this hypothesis of cerebellar plasticity, this study investigates the functional reorganization of the cerebellum using direct electrical stimulation in human.

Twelve patients were recruited. They all had either a tumor or a malformation in the posterior fossa, inside the cerebellum (vermis and/or hemisphere). Per operative motor evoked potential were used to map the posterior cerebellum.

Most of the responses were ipsilateral to the hemisphere stimulated. But we also find contralateral responses. Those responses were all evoked from the healthy hemisphere opposite to the lesion. Motor somatotopy was preserved in the contralateral 
hemisphere. Long-term postoperative clinical evaluation shows that motor deficits remain low to moderate.

This study confirms that movement can be triggered by electrical stimulation of the cerebellar cortex and there is a somatotopic organization within the posterior cerebellum. Moreover, in case of a cerebellar cortical lesion, it is possible to produce controlateral muscle contractions when stimulated the opposite hemisphere to the lesion. This shows some form of compensatory mechanisms. This plasticity may allow motor recovery that is observed clinically .

The cerebellum is one of the major structures of our central nervous system and is a frequent target of tumors or malformations. A better knowledge of its functions is essential for the management of our patients. This study helps to understand that the cerebellum is a plastic structure that can compensate the loss of motor function by reorganizing its eloquent areas.

\section{FP027}

Frameless stereoelectroencephalography (SEEG) in children: a pediatric series from a unique center

Didier Scavarda, Agnes Trebuchon, Mathieu Milh, Gregoire Pech Gourg, Anne Lepine, Nathalie Villeneuve, Nadine Girard, Fabrice Bartolomei (Marseille, France)

Introduction: Precise localization of the epileptogenic zone (EZ) is the goal for all the surgeons involved in epilepsy surgery. Different ways to define this target are used all over the world. SEEG, the gold standard, was developed in France in the 50's and is now used all over the world. Currently, modernization of the operative rooms allows operating under computer control. So we developed an alternative methodology to implant children with deep electrodes, which is secure, decrease the radiations and the operative time and accurate. We report our frameless and simplified methodology of SEEG, its efficacy and safety in children

Material and Methods: We prospectively studied our children investigated for refractory epilepsy in our institution with a simplified frameless implantation methodology that we developed since 2011 for deep electrodes implantations. Our technique is based on a neuronavigation system and a biopsy arm (Stealthstation Medtronic) without any added software. The number of electrodes, the side of implantation, the age of the children, the accuracy of the entry point, the accuracy of the target point, the operative time, the complications and the ability to define the EZ are reported.

Results: During the period from March 2011 to December 2015 we performed 60 SEEG in children. Age varied from 20 months to 21 years old. 40 implantations were unilateral and 20 bilateral. We implanted a total of 637 electrodes with a mean of 10 electrodes/child. Implantation time varied with experience from 12 to $7 \mathrm{mn} /$ electrode. Mean time under general anesthesia is about 240 minutes. Accuracy has varied during this period after modification of our imaging protocol. We found one broken electrode and 3 hemorrhages ( 2 minor/ 1 major). Complication rate/electrode: $0,62 \%$.

Discussion: Our methodology was developed to decrease the amount of radiation to the children and to decrease the time under general anesthesia. These goals were achieved but we needed to perform a CT scan to improve the accuracy. This technique is very intuitive and less expensive for the institutions with the same amount of complications. We did not add any software to plan the trajectories. Finally, accuracy matches positively with the few data available in the literature.

Conclusions: Frameless and simplified SEEG with a biopsy arm is a safe and accurate technique for deep electrodes implantation in children with refractory epilepsy. It decreases the radiations for children. Association of CT scan and MRI in the planning protocol increases the accuracy and safety. Delineating of the epileptogenic is not different from classical SEEG.

\section{FP028}

Early implantation of vagal nerve stimulation in children Jehuda Soleman, Corine Knorr, Christian Schneider, Alexandre Datta, Sussi Strozzi, Gianpaolo Ramelli, Luigi Mariani, Raphael Guzman (Basel, Switzerland; Bern, Switzerland; Bellinzona, Switzerland)

Aim: Data concerning the benefit of vagal nerve stimulation (VNS) in children under the age of 12 years is sparse. In addition these studies include mostly patients above the age of 6 years. We therefore assessed the safety and efficacy of VNS in the children operated on at our institution.

Methods: Retrospective review of patients undergoing VNS implantation, from 2010 to 2015, was undertaken. All patients were operated by the senior authors (R.G. and L.M.), while adjustment of anti-epileptic drug (AED) postoperatively, clinical and electroencephalography (EEG) follow ups were concluded by the referring neurologist. We analyzed operative complications and AED use as well as seizure frequency using the Engels and McHugh classification.

Results: Out of 60 consecutive VNS implantations for therapy refractory epilepsy at our institution, 13 were implanted in children $(<18$ years old $)$. The mean age at the time of surgery was 8 years, while 5 patients (38.5\%) were under the age of 5 years and 2 patients $(15.4 \%)$ above the age of 12 years. There were 7 females and 6 males. All patients suffered from therapy refractory epilepsy, while seven patients $(53.8 \%)$ showed additionally a psycho-motoric retardation. Overall 6 patients (46.2\%) showed a postoperative reduction in their seizure frequency of $<50 \%$ (McHugh and Engels Class III), while in patients under the age of 6 years a reduction in seizure frequency of $<50 \%$ (McHugh and Engels Class III) was seen in $60 \%(n=3)$ of the cases. One third of the cohort $(30.7 \%)$ 
reduced the number of AEDs after surgery, while in patients under the age of 6years a reduction was seen in $20 \%(n=1)$. In one patient ( 8 years old) the VNS had to be removed $(7.7 \%)$ due to infection and was then re-implanted on the contralateral side. The same patient had temporary vocal cord palsy with hoarseness postoperatively.

Conclusions: VNS seems to be a safe therapy in children even under the age of 6 years. Quality of life measured with the Vineland Adaptive Behavior Scale and analogue scale of parental satisfaction will be presented and discussed.

\section{FP029}

Pediatric epilepsy surgery - A single surgeon's experience Catherine Pringle, Vivek Josan (Manchester, United Kingdom)

Introduction: We carried out a retrospective analysis of the first 61 contiguous paediatric patients undergoing epilepsy surgery at our institution by the senior author over a seven year period. All the patients in our study had at least one year follow-up with the epilepsy surgery team. None of our patients underwent invasive monitoring.

Methods: A retrospective case note analysis was carried out, specifically documenting the demographics, histology, complications, length of stay and Engel's score.

Results: 61 patients (32 girls and 29 boys) underwent epilepsy surgery from 2007 to 2014. Age range at time of surgery was 0.6 to 18.6 years. Of these 17 underwent hemispherotomies, 20 had lesional surgery, 21 temporal lobectomies and 3 temporo-parieto-occipital disconnections.

Histology of the lesional cases was: 10 DNETs, 2 cavernomas, 1 angiocentric glioma, 1 glioneuronal tumour, 1 tuberous sclerosis, 2 temporal encephalocoels, 1 neurocytoma, 1 non specific lesion, 1 extra temporal cortical dysplasia.

3 of the hemispherotmies and 1 lesional surgery underwent reexploration.

At one year follow-up the Engel scores were as follows: Engel 1- 36; Engel 2 - 15; Engel 3 - 7 and Engel 4 - 3. 51 of the 61 (84\%) patients had a good (Engel 1 or 2 ) outcome.

3 patients required CSF diversion, 1 patient developed an unanticipated hemiparesis which resolved completely eventually and 3 had postoperative visual field deficits.

Mean length of stay was 11.3 days (3-80 days)

Conclusion: Epilepsy surgery offers good seizure control with minimal morbidity and no mortality in neurologically complex pediatric patients. Longer follow up will be carried out to assess longevity of seizure control. We are in the process of developing invasive monitoring at our center to offer our patients a more comprehensive epilepsy surgical management.

\section{FP030}

Selective dorsal rhizotomy - The Leeds experience over our first 60 cases
John R. Goodden, Alec Musson, Kate McCune, Katie Davis, Ian Smith, Michael Clarke, Raj Lodh (Leeds, United Kingdom)

Introduction: Selective Dorsal Rhizotomy (SDR) aims to improve function and mobility through reduction in abnormal lower limb tone. We present Functional and Quality of Life $(\mathrm{QoL})$ outcomes from the first 60 cases performed in our centre in England. We also report on our experience developing this new service.

Methods: We performed our first SDR procedure in October 2012. Post-operative outcomes are prospectively recorded using a spreadsheet database.

Standardised patient selection criteria were used - spastic diplegia with dynamic spasticity limiting function, no dystonia, typical MRI changes, and GMFCS Level 2 or 3.

SDR is performed via a single-level laminectomy, using intraoperative neurophysiology. Approximately $66 \%$ dorsal rootlets are cut from L1-S1.

All patients had standardised pre \& post op assessments with 3D Gait Analysis, GMFM-66, Ashworth grading, muscle power and range of movement. QoL was assessed using the CPQoL questionnaire.

Results: Sixty patients have had SDR (40 male, 20 female). Mean age at surgery was 6.75 years (range 2.6-14.4). Patients were GMFCS 2 (28) or GMFCS 3 (32). Mean follow-up is 655 days (range 178-1291).

Over time the patient pathway has improved with shorter operation duration \& hospital stays, better patient information, improved analgesia and developments in physiotherapy.

All patients have reduction in tone after SDR. GMFM-66 results demonstrate improvement.

Quality of Life was improved in all domains, with $>40 \%$ improvement in Participation \& Physical Health in the first year.

There were no significant complications - specifically no paralysis, numbness, or bladder/bowel dysfunction.

Conclusion: SDR is a safe and effective treatment for appropriately selected patients with spastic diplegic cerebral palsy. Our service has developed and grown as we have gained experience over these 50 cases. Important improvements in function and QoL are demonstrated.

\section{FP031}

Rational of optic nerve decompression in optic nerve injury

Ashok Kumar Mahapatra (Bhubaneswar, India)

Introduction: Optic Nerve Injury is a rare condition and reported incidence between 0.05 to $1.5 \%$ of all head injury. The pathogenesis and management still remains controversial. Hence, there is need for proper review. 
Material: Over the last 32 years, author has treated more than 1000 cases of optic nerve injury and time to time published the data and laid down the guidelines for management. Unilateral injuries cases are $85 \%$ and in $15 \%$ cases, it may be bilateral. In $90 \%$ cases, the visual deterioration is immediate following the injury and $10 \%$, it is delayed in onset. VEP is a very good diagnostic and progestic test and helpful in determining optimal outcome. Repeated normal VEP indicates a good outcome. Optic carnal decompression is not indicated for 3-4 weeks, and conservative management by using corticosteroid. More than $50-50 \%$ cases show spontaneous recovery, which starts something between 48 hours to 3 weeks and continues for longer period. In case a patient does not show any improvement in 03 weeks time, his chances of onset of recovery is remote. Optic canal decompression was carried out in selective cases when the patient shows some improvement and then remains static. In such a situation, optic nerve decompression helps in further improvement in all $100 \%$ cases, however, none of the patients those who underwent surgery, had $100 \%$ recovery. All the $10 \%$ patients those who had $100 \%$ recovery, were managed conservatively with steroid.

Conclusion: Overall improvement observed in $55-59 \%$ and in limited cases of optic nerve decompression is indicated. Patients those who initially improved minimally and remained static there after, always benefitted from surgery decompression. VEP is a very good diagnostic and progestic investigation. Details of the data will be discussed.

\section{FP032}

\section{Surgical treatment of intraventricular hemorrhage in newborns}

Ivan Protsenko, Leonid Marushchenko, Myroslava Marushchenko (Kiev, Ukraine)

Introduction: The purpose of research was to improve the efficiency of the treatment of newborns with intraventricular hemorrhage (IVH).

Methods: The research was based on analysis of the results of treatment of 119 newborns with perinatal IVH. Grade I IVH (L.Papile, 1978) were diagnosed in $11(9.2 \%)$ cases, Grade II IVH -in $24(20.2 \%)$, Grade III IVH - in $58(48.7 \%)$ children, Grade IV IVH -in 26 (21.8\%). For comparative monitoring of effectiveness of different types of treatment 56 newborns with IVH, who received only conservative therapy were taken additionally.

Results: Surgical procedures and operations have been executed in newborns with IVH Grade III and IV. There were repeated lumbar punctures, ventricular punctures, external ventricular drainage, ventriculosubgaleal drainage, the needle removal of intracerebral hemorrhage, subcutaneous implantation of a ventricular reservoir and shunt surgery. The tactics of treatment was based on grade and form of hemorrhage, the degree of disorders of liquor circulation and severity of increased intracranial pressure. The newborns with Grade I and II IVH on early stages generally didn't need active neurosurgical care and treatment was limited by the conservative therapy and lumbar puncture. When communicating progressive hydrocephalus developed despite to medical therapy, children needed shunt operation. Children with Grades III and IV IVH required active neurosurgical care (external ventricular drainage) from the early stages of the disease. Formation of noncommunicating and communicating hydrocephalus was direct indication to shunt operation. In cases when active surgical tactics has been applied at Grade III IVH and at Grade IV the mortality has decreased by $31.5 \%$ and by $55.7 \%$ accordingly. Frequency of progressive hydrocephalus has been reduced by $36.7 \%$.

Conclusion: Early active neurosurgical treatment can reduce the level of mortality in newborns with perinatal IVH from $43.7 \%$ to $16.2 \%$ and necessity of shunt operations from $66.7 \%$ to $42 \%$.

\section{FP033}

A method for the creation of normative pediatric skull models: a pilot study

Manon Tolhuisen, Guido de Jong, Jene Meulstee, Ferdi van der Heijden, Hans Delye (Nijmegen, the Netherlands; Enschede, the Netherlands)

Introduction: Today, no objective standardized planning procedure exists for the preoperative planning of open cranial vault reconstruction of patients suffering from craniosynostosis. Therefore, the reconstruction is subjective and dependent on the experiences and preferences of the surgical team. The use of a normative skull model, which will serve as a reference, will enhance the standardized planning procedure. A method was developed to create a normative skull model.

Methods: The presented method was applied on five CT-scans. First, the skull is segmented using $167 \mathrm{HU}$ as threshold. Secondly, a tetrahedral mesh of the skull is created and registered using four reference points. The sella turcica-nasion line is placed on the y-axis, with the center of the sella turcica as origin. Both anterior clinoid processes are used to place the skull horizontally. Finally, ray casting is applied to obtain metrical data.

Results: A normative skull model was created based on metrical data obtained from five $\mathrm{CT}$ scans.

Conclusion: The presented method was able to create a normative skull model, using five CT data sets containing skull images. Therefore, this method is suitable for the creation of normative skull models and provides the first step in the creation of an objective fully automated planning procedure for cranial vault reconstructions. 


\section{FP034}

Neonatal hypoxia ischemia in rat disrupts the developmental time course of doublecortin release in the cerebrospinal fluid

Catherine Bregere, Urs Fisch, Sebastian Lieb, Martin Sailer, Laurie Chicha, Fabienne Goepfert, Thomas Kremer, Raphael Guzman (Basel, Switzerland)

Introduction: Doublecortin (DCX) is often used as a marker of neurogenesis in immunohistochemical (IHC) stainings of adult rodent brains. Using a recently developed immunoassay that enables objective quantification of DCX in tissues and body fluids, we examined whether DCX in the cerebrospinal fluid (CSF) may be an indicator of endogenous neurogenesis. This question was addressed in the rat model of neonatal hypoxia-ischemia (HI), a brain injury documented to stimulate neurogenesis.

Methods: HI was elicited at postnatal day (P) 7 in SpragueDawley rats via ligation of the right common carotid artery and 40 minutes exposure to $8 \% \mathrm{O}_{2}$. Control animals received a sham surgery without $\mathrm{HI}$. CSF was collected serially at P5 and P10, or at P10 and P15. Bromodeoxyuridine (BrdU) was administered from $\mathrm{P} 7$ to $\mathrm{P} 9$, and P10 brains were processed for IHC analyses.

Results: In sham-exposed neonates, a significant and continuous drop in the mean concentration of DCX in the CSF (CSF-DCX) occurred between P5 and P15. In HI-exposed neonates, CSF-DCX rose significantly between P5-P10, but declined between P10-P15; yet, at P15, CSF-DCX remained significantly higher in HI than in control neonates. In the P10 HI group, CSF-DCX and stroke severity correlated positively. HI caused a significant increase in DCX immunointensity, and in the number of BrdU positive cells in the ipsilateral neurogenic niches. Of note, most DCX expressing cells were negative for cleaved caspase-3, a cellular death marker, in both groups. In vitro, DCX could be quantified in the cell culture medium of differentiating rat neural stem cells, and appeared to emanate from living cells.

Conclusions: Neonatal HI brain injury remarkably disrupts the developmental time course of DCX release in the CSF. We hypothesize that DCX in the CSF reflects, in part, the neurogenic response in the brain after the insult.

\section{Session 5: Oncology}

\section{FP035}

\section{ALA use in pediatric brain tumors}

Jonathan Roth, Shlomi Constantini (Tel Aviv, Israel)

Introduction: Over recent years, 5-amino-leuvolinic acid (5ALA) has been increasingly used for resection guidance in adult primary brain tumors, especially when resecting highgrade gliomas. Its efficacy improving extent of resection, and progression free survival has been proven in phase III studies. Amongst pediatric patients, despite the importance of extensive resection of both low and high-grade tumors, the use of intraoperative fluorescence has been limited to about 120 cases with inconsistent outcomes. We describe our experience and intraoperative finding amongst children with various brain tumors that were given 5ALA prior to tumor resection. Methods: Since October 2014, data regarding intraoperative findings amongst children that received 5ALA prior to tumor resection were prospectively collected. Inclusion criteria included any intracranial tumor among children 318 years of age. Parental consent was given in all cases. Data included intraoperative findings (regarding fluorescence of the tumor), as well as postoperative follow up and documentation of complications.

Results: 12 children were included with a wide pathological spectrum: pilocytic astrocytoma (4), medulloblastoma (2), and PXA, DNET, supratentorial PNET, hemangiopericytoma, hemangioblastoma, and ganglioneuroblastoma (1 each). 9 children had an intraoperative evaluation - none had a positive fluorescence. None of the 12 patients suffered 5ALA related complications or abnormal liver enzymes. There was 1 death (30 days post operatively) - related to an extensive tumor recurrence in a large cell medulloblastoma.

Conclusion: Despite the heterogeneity of pathologies in the current series, none of low - and high-grade tumors showed a positive intraoperative fluorescence. Reviewing the literature, the role of 5ALA in guidance of tumor resection in pediatric brain tumors seems to be limited.

\section{FP036}

Immunohistochemical evaluation for the pathogenesis of intracranial germ cell tumors: expression of pluripotency and cell differentiation markers

Tadashi Miyagawa, Hisayuki Murai, Yasuo Iwadate, Naokatsu Saeki (Matsudo, Japan; Chiba, Japan)

Introduction: Primary intracranial germ cell tumors (iGCT) are rare neoplasms that occur in children and adolescents. This study examined both the pathogenesis and the origin of these tumors, as it has been hypothesized that they originate from a totipotent primordial germ cell.

Materials and Methods: We applied recent knowledge from gonadal germ cell tumors and analyzed expression of a wide panel of stem cell-related proteins (C-KIT, OCT-3/4 (POU5F1), NANOG, SOX2, CD30 and PLAP). Expression shown by immunohistochemistry was analyzed in 12 children and young adults with iGCT, contributing to a careful description of these unusual tumors and adding to the understanding of pathogenesis. 
Results: Immunohistochemistry showed $5 / 5$ positive in Oct3/4, 5/5 in NANOG, $9 / 9$ in C-KIT and $1 / 5$ in SOX2. All cases that showed positive staining in Oct $3 / 4$ or NANOG indicated that the tumors contained germinoma or embryonal carcinoma component. Immunohistochemistry revealed that stem cell related proteins were highly expressed in iGCT, and many similarities were detected with their gonadal equivalents, including a close similarity with primordial germ cells.

Conclusion: The expression of genes associated with embryonic stem cell pluripotency in CNS germ cell tumors strongly suggests that these tumors are derived from cells that retain, at least partially, an embryonic stem cell-like phenotype, which is a hallmark of primordial germ cells. In addition, these data can be applied to stratify iGCT for treatment strategy in order to provide good survival rate and good functional outcome.

\section{FP037}

\section{Medulloblastoma DAOY cell line: advances in proteome} characterization

Luca D'Angelo, Gianpiero Tamburrini, Luca Massimi, Paolo Frassanito, Claudia Martelli, Marta Barba, Francesca Iavarone, Concezio Di Rocco, Wanda Lattanzi, Massimo Castagnola, Massimo Caldarelli, Claudia Desiderio (Rome, Italy; Hannover, Germany)

Introduction: Medulloblastoma is the most commonly investigated pediatric brain tumor. More and more studies are devoted to its molecular characterization by in vitro proteomic analysis following the widespread, classical bottom-up approach. The new data on DAOY cell culture entire proteome obtained by a LC-MS top-down platform are here discussed and compared with the state of proteomics in medulloblastoma.

Methods: Intracellular proteome of DAOY cells cultured in serum-free medium have been analyzed by HPLCESI-LTQ-Orbitrap XL tandem mass spectrometry platform without protein enzymatic digestion. Proteins and peptides characterization was performed either by manual or software elaboration of MS/MS fragmentation data with high degree of confidence.

Results: The analysis of DAOY cell lysates in acidic solution by top-down proteomic approach allowed to identify naturally occurring small proteins, proteins fragments and biologically active peptides in the undigested form. Together with already reported proteins, this approach permitted to disclose 53 small proteins and peptides such as beta- and alpha-thymosins, several histones, protein S100 A-11, profilin-1 and dolichyldiphosphooligosaccharide-protein glycotransferase subunit 4 never identified in medulloblastoma cells up to date (25 proteins). Most of the characterized proteins and peptides are involved in biological pathways connected to cell motility, proliferation, invasion and evading surveillance mechanisms.

Conclusions: The proteomic data obtained by top-down approach well complement and update the medulloblastoma proteomic profile reported in literature contributing to elucidate the molecular processes possibly involved in the genesis and progression of this tumor and/or identifying potential biomarkers or molecular targets for future therapies. This strategy allows the investigation of small proteins and peptides particularly disclosing the presence of post-translational modifications and isoforms that are often at the basis of tumorigenesis.

\section{FP038}

Immunohistochemical analysis of Galectin-3 in gliomas: a tissue microarray study with a comparison of three commercial antibodies targeting different epitopes

Ricardo Santos de Oliveira, Bruna Riedo Zanetti, Isabela Martins, Deise L. Chesca, Benedicto Oscar Colli, Carlos Gilberto Carlotti Jr, Helio Rubens Machado, Luciano Neder (Ribeirao Preto, Brazil)

Introduction/Objective: Galectins are a family of 15 animal lectins that bind specifically $\beta$-galactoside residues. They have important roles in a variety of cell functions, such as cell-cell interactions, matrix adhesion and transmembrane signaling. Among them, galectin-3 (Gal-3) is of particular interest in gliomas. However, conflicting results have been published about the immunohistochemistry (IHC) expression of Gal-3 on these tumors, most probably due to different antibodies and protocols used.

Material and Methods: We investigate the IHC expression of Gal-3 in glioblastomas (GBMs, $n=31$ ) and grade II and III oligodendrogliomas (OGs, $\mathrm{n}=21$ ) as well as mixed tumors (so called "oligoastrocytomas", $\mathrm{n}=13$ ) using tissue microarray (TMA) platform. For such we used commercially available antibodies against 3 distinct epitopes of Gal-3 (NH2-terminal, C-terminal and full-length Gal-3 molecule). Two distinctive tumor cores $(2 \mathrm{~mm})$ were chosen in each case and the final score for Gal-3 expression was calculated from the mean IHC expression of both cores, according a semiquantitative evaluation: 0:no labeling; $1+$ :up to $25 \% ; 2+: 25 \%$ to $75 \% ; 3+$ $:>75 \%$. As expected the antibody against the full-length Gal3 molecule showed the most robust labeling and, therefore, used for Gal-3 evaluation.

Results: Sixteen out of 31 GBMs showed stronger Gal-3 expression $(>2+)$, although 6 GBMs did not show any labeling as well as 19 out 21 OGs and 9 out 13 mixed tumors. Hence, the Gal-3 expression is significant expressed in GBMs in comparison with OGs $(\mathrm{p}<0.001)$. Interestingly, in contrast of normal brain vessels, neoplastic vessels with microvascular proliferation of glomeruloid features did not show Gal-3 
expression. The mean final score for GBMs, OGs and mixed tumors was $2.25,0.24$ and 0.3 , respectively.

Conclusion: The IHC using the antibody against the fulllength of Gal-3 molecule may be helpful in the differential diagnosis between GBMs and anaplastic OGs.

Support: FAPESP (Grant No. 2014/02398-4)

\section{FP039}

Intra-CSF drug delivery for the treatment of leptomeningeal metastases: patient selection and risk profile

Ibrahim Jalloh, Maria Cartmill, Michael Vloeburghs, Tim Jaspan, Sophie Wilne, Richard Grundy, David Walker, Donald Macarthur (Nottingham, United Kingdom)

Introduction: Combination chemotherapy with systemic and intra-CSF agents enables cytotoxic concentrations to be achieved within both the CSF and brain parenchyma potentially allowing control of bulk disease, treatment of leptomeningeal deposits and prevention of CSF dissemination. Concern regarding complications of CSF access devices, disturbed CSF dynamics and potential myelotoxicity has led to reluctance to pursue intra-CSF treatments. We review our experience of intra-CSF chemotherapy delivery.

Methods: Intra-CSF chemotherapy was delivered either via an intraventricular reservoir or a lumbar intrathecal catheter. Gadolinium contrast studies were used to confirm adequate CSF distribution throughout the brain and spine when there was concern about potential CSF flow obstruction.

Results: 19 children (9F, 10M) aged 2 months to 17 years were treated between 2008 and 2015; 12 via Ommaya reservoirs, 5 via lumbar ports and 2 via a combination of the two. Histology included medulloblastoma (4), ependymoma (4), supratentorial PNET (2), ATRT (2), sarcoma (2), choroid plexus carcinoma (1), pineoblastoma (1), sarcoma (2) and haematological malignancy (3). Radiological response was achieved in 11 children (58\%). There were 7 device-related adverse events (CSF leak, infection). 13 patients, many of whom were treated with palliative intent, have died but 6 are still alive, either in remission or with stable disease.

Conclusions: Combined Intra-CSF and systemic chemotherapy is effective although not always straightforward due to device-related complications. Appropriate selection of patients will be determined by histology, location and distribution of disease and the extent to which other options have been utilised or exhausted. We feel that for appropriate patients intra-CSF chemotherapy can be delivered via equipment familiar to all paediatric neurosurgeons with an acceptable risk profile and potential significant patient benefit.

\section{FP040}

Antero lateral brainstem gliomas: our surgical experience with the posterior petrosal approach in 10 children
Uli Barcik, Fernando Carceller Benito, Javier Saceda (Madrid, Spain)

Objective: Focal anterolateral midbrain and pontine tumors are very rare. They may be reached through a combined petrosal approach which combines a subtemporal and a transtentorial presigmoid avenue, an approach that has the advantage of a short distance and a direct line of sight to the anterolateral brainstem. We share our experience in children and show technical details on how to adapt the craniotomy depending on the precise location of the tumor.

Methods: The authors conducted a retrospective study of 10 pediatric patients since 2004 undergoing a posterior petrosal approach in our unit. We collected pre- and postoperative data, including presentation, imaging findings, pathological results, operative details, complications and outcomes. The craniotomy was extended higher when the tumor was located more caudally in order to gain better access.

Results: There were 6 boys and 4 girls with a mean age of 4 years and 11 months. The posterior petrosal approach was the sole skull base approach in 10 children. In 6 cases the right side was accessed whereas in 4 cases the left side. The craniotomy was extended higher in 6 patients. Complications occurred in 3 patients but were transient in nature. There was no perioperative mortality. A gross-total resection was achieved in 2 patients, subtotal resection in 4, and partial resection in 4 was performed. Pathological results: Astrocytoma (WHO Grade 3) in 5 cases, Astrocytoma II in 2 cases, Pilocytic Astrocytoma in 1 cases, Oligodendroglioma (WHO Grade 3) in 1 case, Glioblastoma multiforme in 1 case, Mean follow-up time in the children which had a benign tumor was 26 months. 7 patients died.

Conclusions: A petrosal approach utilizes a wide opening of the tentorium and the option of supratentorial retraction of the cerebellum to provide an excellent angle of approach to lesions involving the anterolateral aspect of the brain stem in children.

\section{FP041}

A report of 33 cases of intracranial tumors diagnosed during the first year of life

Nathalie Chivoret, Gregoire Pech-Gourg, Laurence Haddi, Armando Paz Paredes, Didier Scavarda (Marseille, France)

Introduction: Intracranial tumours in the first year of life were considered rare. Our knowledge about CNS tumours in this age group is still limited.

Method: During an eleven year period, 33 infants aged less than 1 year with intracranial tumours were diagnosed at the department of pediatric neurosurgery in Marseille. The initial diagnosis was performed with CT-scan, MRI or ultrasound examination. 
Results: We observed a male preponderance and a relatively higher incidence at 6 and 9 months. The most frequent histological types in this serie were astrocyomas (6 cases), ATRT (5 cases), ependymomas (4 cases), medulloblastomas ( 2 cases), papillomas (3 cases), gangliogliomas (3 cases), teratomas (3 cases). $51 \%$ of these tumours were benign.

The most regular symptoms at clinical presentation were intracranial hypertension (15 cases), seizure disorders (4 cases) and ocular symptoms (4 cases).

Infratentorial localization was observed in 17 cases and supratentorial localization in 16 cases.

A surgical resection was done in 27 cases. Radical resection was done in 16 patients. In one case only a biopsy procedure was performed. There were no operative deaths.

A specific treatment of hydrocephalus was required in 15 cases (shunt in 10 cases, ventriculocisternostomy in 5 cases). 17 patients received chemotherapy and 4 patients radiotherapy.

The median follow-up was 48 months. Seven deaths (21\%) were recorded. Three patients were lost to follow-up.

At last follow-up, 26 patients were alive. We observed in this group of survivants long term morbidity in 11 cases (42\%): 8 patients with neurological deficits, 2 patients with severe psycho-motor retardation and 2 patients with epilepisa.

Conclusion: $79 \%$ of our population is alive. More than $50 \%$ per cent of patients present no neurological disorders in long term follow-up. Furthermore, no operative mortality was observed. These facts confirms that surgery should be performed safely in this period of age and without delay.

\section{FP042}

Evolving trans-sphenoidal surgical management of paediatric craniopharyngiomas: a single surgeon's experience

Soumya Mukherjee, Asim Sheikh, Gnanamurthy Sivakumar, John Goodden, Paul Chumas, Atul Tyagi (Leeds, United Kingdom)

Introduction: Craniopharyngioma develops from residual cells of Rathke's pouch. It represents $2.5-4 \%$ of all brain tumours, and half present in childhood with a peak incidence at 5-10 years. Surgical resection is performed to prevent hydrocephalus, bitemporal hemianopia and endocrine imbalance. Operative approaches include craniotomy by frontotemporal or trans-callosal routes and more recently transsphenoidal (endoscopic and microscope assisted). We present our experience using the trans-sphenoidal approach.

Methods: Retrospective analysis was undertaken for all paediatric cases of craniopharyngioma operated using the transsphenoidal approach between January 2000 and January 2014 at a single institution. Data on demographics, operative details, complications, and extent of resection based on comparison of pre- and post- operative magnetic resonance imaging (MRI) were collected.
Results: During the study period 8 cases were operated: three trans-sphenoidal microscopic and five trans-sphenoidal endonasal. Male-to-female ratio was 5:3 and mean age was 13 years (range, 8-17 years). 4 of 8 cases had been previously operated with craniotomy. All cases used a combined neurosurgical-otolaryngology approach and frameless stereotactic neuro-navigation. Gross total resection $(>95 \%$ tumour volume) was achieved in all 8 cases. Post-operative complication rate was $25 \%$ (2/8) including cerebrospinal fluid (CSF) leak (1/8, $12.5 \%)$ and diabetes insipidus $(1 / 8,12.5 \%)$. At two-year followup there has been no instance of recurrence.

Conclusion: Gross total resection can be consistently achieved with the endoscopic trans-sphenoidal approach. Neuronavigation may improve safety of the operation. Increasing endoscopic experience can lead to adoption of a pure endoscopic approach, as in our institution.

\section{FP043}

Pre-operative neutrophil lymphocyte count ratio (NLCR) in predicting the histological grade of pediatric glial tumors: a preliminary study

Fozia Saeed, Jamie Wilson, Atul Tyagi, Sivakumar Gnanamurthy, John Goodden, Paul Chumas (Leeds, United Kingdom)

Introduction: Neutrophil-Lymphocyte Count Ratio (NLCR) is an established prognostic marker for renal, lung and colorectal carcinomas, and has been suggested to be predictive of histological grade in adult intrinsic primary brain tumours. The aim of this investigation was to investigate whether the NLCR in paediatric intrinsic tumours can be predictive of the final histological grade.

Methods: Retrospective analysis at a single centre. Patients less than 18 years old at the time of surgery who underwent tumour-related procedures from 2006-2015. Craniopharyngiomas, recurrent tumours and metastases were excluded. Pre-operative NLCRs (collected before the diagnosis of intracranial pathology, and before administration of steroids) were matched with histological diagnosis for each patient. Post-operative NLCR was also recorded where possible. Results: 214 paediatric procedures were recorded in total. 84 patients with a diagnosis of primary intrinsic tumours had preoperative NLCR that could be matched to final histological grade. WHO Grade 1 and 2 tumours (grouped together, $\mathrm{n}=50$ ) had a mean NLCR of 2.57 compared to a mean NLCR of 3.63 for Grade 3 and 4 tumours ( $n=34$ ), which was not statistically significant $(\mathrm{p}=0.086$ ). One-way ANOVA showed no statistical NLCR significance between each grade of tumour. The NLCR for Medulloblastoma was significantly higher (7.86) compared to the low grade cohort $(\mathrm{p}=0.002)$. Post-operative NLCR showed no significance between the low grade compared to the high grade group $(\mathrm{p}=0.92)$, or between each grade. 
Conclusion: The evidence to support or refute the effectiveness of NLCR to predict histological diagnosis in paediatric intrinsic tumours has not been published. Our preliminary results suggest no difference exists between the mean NLCR between low grade and high grade tumours, but significant differences may exist between certain subgroups. Final analysis and comparison of all subgroups will be presented at the conference.

\section{FP044}

\section{Pineal cysts: neurosurgical perspective}

Hrvoje Jednačak, Jakob Nemir, Josip Paladino, Goran Mrak, Sibila Nanković, Branka Bunoza, David Ozretić (Zagreb, Croatia)

Background: Pineal cysts and pineal tomors represent most of the pineal region lesions. We report our experience with treatment of patients with large pineal cysts.

Methods: We performed retrospective study which included 10 patients with pineal cyst diagnosis. All pediatric patients with diagnosis of pineal cyst surgically treated in period from January 2002 to December 2014. in our Department were included. The study included 8 female and 1 male patients. All patient had large simple pineal gland cysts, with compressive effect. All patients had headache, while four of them had epileptic phenomena-primary generalized seizures. Patients had common electroencephalography (EEG) pattern with paroxysmal discharges of $3 \mathrm{~Hz}$ (or more than $3 \mathrm{~Hz}$ ) spike-andwave complexes. Operation using sitting position with supracerebellar infratentorial approach was performed in all patients. In most of our patients indication for the operation was established based on the size of the cyst ( $15 \mathrm{~mm}$ or more), with the signs of compression on the quadrigeminal plate and compression of the surrounding veins, which could result in headaches, seizures and EEG changes.

Result: Pathohistological analysis revealed pineal gland cysts in all patients. Following surgery clinical condition improved in all patients (seizure-free and headaches significantly decreased). Other symptoms including diplopiae, nausea, vomiting, vertigo as well as blurred vision also disappeared. There were no complications after surgical procedures.

Concusion: Our hypotheses are that mass effect on the surrounding veins that affects normal perfusion, compressive effect on the quadrigeminal plate and the aqueduct of the midbrain, as well as secretion disturbances of anticonvulsive agent melatonin can be involved in the pathogenesis of seizures and headaches. We suggest to perform high resolution brain MRI with special demonstration of pineal region in all young patients that have seizures and specific EEG changes.

\section{FP045}

The role of surgery in non-NF-associated pediatric optic pathway gliomas (OPG)
Laura-Nanna Lohkamp, Peter Manley, Mark W. Kieran, Nicole Ullrich, Liliana C. Goumnerova (Berlin, Germany; Boston MA, USA)

Introduction: The surgical management of pediatric OPG is variable as the indications and type of procedure are adapted individually to the tumor behavior and clinical course. Thus surgery might represent a crucial factor for outcome variation. This study addresses the overall role of surgery in terms of establishing any paradigm that would approach surgical guidelines in pediatric non-NF-associated OPG.

Methods: This study comprises a retrospective review of all children with diagnosed OPG who were treated and followed in a single institution between 1981 and 2015.

Results: 122 children with diagnosed non-NF-associated OPG were identified, including 69 males and 53 females. The average age at diagnosis was 9.6 years (median; mean: 6.3 years). Radiographic measurement of the tumor revealed a tumor size $>3 \mathrm{~cm}$ in $50 \%$ of the children. The median follow up was 6 years. 89 children received initial surgery, of which 7 were complete and 48 subtotal resections. An initial diagnostic biopsy was performed in 27 of the children. Initial hydrocephalus-directed surgery was performed alone in 7 children and combined with tumor surgery in 31 children. Surgery as second line therapy was required in 7 children, including 5 tumordirected procedures. 21 children were treated with surgery alone. In total 247 surgeries were performed with an overall complication rate of $2 \%$. The overall survival rate in operated children was $85.4 \%$ compared to $92 \%$ of children initially treated with chemotherapy.

Conclusion: Surgery was shown to be beneficial in terms of tumor mass reduction and avoidance of hydrocephal complications in addition to single treatments in 21 children. Given the low complication rates compared to chemotherapyinduced side effects surgery remains the first-line treatment of choice in pediatric OPG with initial hydrocephalus and tumors exceeding a size of $3 \mathrm{~cm}$.

\section{FP046}

Pediatric diffuse intrinsic pontine gliomas: to or not to biopsy

Ganesalingam Narenthiran, Concezio Di Rocco (London, United Kingdom; Hannover, Germany)

Introduction: Paediatric DIPGs invariably leads to fatality with $90 \%$ mortality at 18 months with no change in survival over the last 30 years. Currently there is no role for surgical excision in the management of DIPG however, biopsy has been performed for research or stratifying management based on hitological or molecular profiles. Nevertheless, biopsy of DIPG remains controversial. The aim of this survey was to gauge the current attitude amongst neurosurgeons towards biopsy of paediatric DIPG. 
Methods: Following a two-week detailed discussions on the ethics of biopsying DIPG in the Neurosurgery Research Listserv (NRL) an electronic survey on biopsyingpaediatric DIPG was circulated to the members (29/05/2015 to $12 / 06$ / 2015). To the discussions there was input from pathologists, ethicists and philosophers. The Survey had ten questions related to biopsying of DIPG. The survey was built using "Google Form". The data was collected in "Google Form" and converted to MS Excel. The data was analysed using the statistical software Stata.

Results: The Neurosurgery Research Listserv has 730 members. There were 40 responses (5.5\%). Of them 27 (68\%) from senior consultant neurosurgeons.

Eight-five percent of the responders though biopsyinginorder to stratify treatment based on molecular profiling ethical.. Eighty-three percent thought it should be performed in conjunction with a research protocol. Forty-seven percent thought biopsying of paediatrci DIPG should be organized under the oversight of international societies.

Conclusion: Biopsyingpaediatric DIPG remains controversial however the attitudes might be changing in view of the potential for the knowledge of molecular biology of tumous impacting the management and outcome. Full results of the survey would be presented at the meeting.

\section{FP047}

Ten years review of posterior fossa cerebellar pilocytic astrocytoma in children

Alexandru Szathmari, Federico Di Rocco, Pierre-Aurélien Beuriat, Blandine Grassiot, Anne Jouvet, Carmine Mottolese (Lyon, France; Bron, France)

Introduction: Although cerebellar pilocytic astrocytoma (CPA) of the posterior fossa (PF) is a benign tumor the resection of the associated cyst wall is still debated. We reviewed 43 patients with a CPAoperated between 2006 and 2015 and analyzed the results focusing on the quality of the surgery.

Patients studied: We reviewed 144 patients with PF tumor operated on in our unit between 1 January 2006 and 31 December 2015. From these, 43 were CPA. Al patients had preoperative cranio-spinal MRI. $80 \%$ ofpatients had primary VCS before surgery. One patient was already operated twice before (first in 1993). All patients were operated by suboccipital approach in sitting position or prone when contraindicated. All patients were followed at 3, 6 than 12 month in the first year and once year thereafter for at least 5 years.

Results: A complete macroscopicremoval was achieved in all patients and verified by the postoperative MRI. In all cases but one, removal of the cyst wall was done even if there was no post-gadolinium enhancement at preoperative MRI. The neurological results are good with no major cerebellar sequels. Slight residual dysmetria was recorded in $20 \%$ of patients and an ataxia in $2 \%$ at the follow-up more than 1 year after surgery. There were 2 local ( 1 no cyst removal) and 1 metastatic recurrences $(7 \%)$. Complete remission was obtained after $2^{\text {nd }}$ surgery at follow-up ( 3 and 6 years) for the local recurrences. The metastatic one is still in slow progression despite chemotherapy.

Conclusion: In our experience, the removal of the cyst wall warrants a complete removal of the CPA and lowers the rate of recurrence without major sequels. Local recurrences can be managed with good results only by surgery. Metastatic evolution of the disease is still possible despite complete removal. A long followup is thereforemandatory.

\section{FP048}

Pediatric infratentorial ganglioglioma: results of a series of 33 children and literature review

Stephanie Puget, Awad Alshehri, Kevin Beccaria, Thomas Blauwblomme, Giovanna Paternoster, Timothé De Saint Denis, Syril James, Christelle Dufour, Franck Bourdeault, Pascale Varlet, Christian Sainte-Rose, Michel Zerah (Paris, France; Villejuif, France)

Purpose: Pediatric infratentorial gangliogliomas are exceedingly rare tumors, thus the factors affecting their outcome are poorly understood and their optimal management has still to be defined. Methods: We reviewed the literature on pediatric gangliogliomas with a focus on those located in the posterior fossa to study treatment and outcome data. We added to this review the results of 33 children treated for this tumor in our institution.

Results: We found 100 and 80 cases of brainstem and cerebellar pediatric ganglioglioma respectively in our literature review. The surgical management varied from biopsy to gross total resection and adjuvant treatment was given after incomplete resection or at time of progression. A gross total resection should be attempted to remove the contrast enhancing part of the tumor, which may be possible in most of the cerebellar gangliogliomas and some of the brainstem lesions. The cervicomedullary ganglioglioma seems to be the most infiltrative and least amenable to complete resection. Chemotherapy has a limited role and BRAF mutation was reported in 38 to $54 \%$ of cases. The use of radiotherapy exposes the patient to risk of malignant transformation and should be reserved for unresectable tumors which progress.

Conclusion: Pediatric posterior fossa gangliogliomas are rare and challenging tumors due to their frequent infiltrative component involving the brainstem. To date, adjuvant therapy has a limited role that may evolve with time thanks to the use of targeted therapies against BRAF mutation. The surgical resection of well-defined contrast enhancing parts should be 
attempted even in staged surgeries and balanced with the risks of neurological deterioration.

\section{FP049}

\section{True aqueductal tumors: a unique entity}

Jonathan Roth, Kaisorn L. Chaichana, George Jallo, Giuseppe Mirone, Giuseppe Cinalli, Shlomi Constantini (Tel Aviv, Israel; Baltimore MA, USA; Naples, Italy)

Background: Pure aqueductal tumors (AT) differ from pineal region and tectal/tegmental tumors in that they are epicentered within the aqueduct. Nevertheless, these tumors are rarely described as a separate type of tumor, and are often grouped with other lesions located in the same vicinity. The present multicenter study focuses on our experience treating patients with pure AT.

Methods: Data from three large tertiary centers was collected retrospectively, including presenting symptoms, treatment paradigm, surgical approaches, pathology, and outcome.

Results: Between 1999 and 2013, 16 patients with AT were diagnosed and treated at the three tertiary centers. Ages at presentation ranged from 5.5 to 57 years. 13 patients presented with hydrocephalus-related symptoms, and two were identified incidentally. 13 patients underwent an endoscopic third ventriculostomy, and two of these underwent a simultaneous endoscopic biopsy (1 grade II ependymoma, 1 non-specified low-grade glioma). Two others underwent shunt placement. Three patients underwent resection due to tumor progression. Pathologies included GBM, glioneural tumor, and ependymoma grade II. All non-resected tumors remained stable or grew only minimally.

Conclusions: Aqueductal tumors are a rare entity that usually presents with obstructive hydrocephalus. Treatment includes primarily CSF drainage (preferably via an ETV). Simultaneous endoscopic biopsy may be done in selected cases. Tumor resection should be reserved for growing tumors; the trans-fourth ventricular or trans-choroidal approaches are probably safer than other approaches used to reach the tectal region.

\section{FP050}

\section{Incidental brain tumors in children - Management} options

Marek Mandera, Mikolaj Zimny, Dominika Czachor (Katowice, Poland)

Introduction: Increased availability of the neuroimaging has lead to more frequent detection of the incidental findings also known as incidentalomas. The aim of our work was to analyse CNS incidentalomas, which were treated surgically, on the basis of the data gathered by the Department of Pediatric Neurosurgery, Medical University of Silesia in Katowice and discuss possible therapeutic options.
Methods: We performed a retrospective analysis of the pediatric patients with primary brain tumors categorized as the brain incidentalomas, which were admitted to our hospital between January 2010 and August 2015. We defined as incidentalomas such brain lesions found on MRI, which were asymptomatic or were not related to symptoms, which were the reason for neuroimaging.

Results: Of 375 hospitalized children treated surgically for brain tumor in this period 9 were qualified as incidentalomas $(2.4 \%)$. The median age of the patients at the time of diagnosis was 10 years. Four of the patients $(44.4 \%)$ were boys and five $(55.6 \%)$ were girls. Reasons for the neuroimaging in these patients were head trauma or headache $(22.2 \%)$, behavior changes $(22.2 \%)$, development delay $(22.2 \%)$ and intensified diagnosing or routine control $(33.3 \%)$. Size of the lesions extended between $10 \mathrm{~mm}$ and $60 \mathrm{~mm}$ in the longest dimension. The tumor was located in the thalamus $(11.1 \%)$, frontal lobe $(11.1 \%)$, temporal lobe $(11.1 \%)$, lateral ventricle $(22.2 \%)$, fourth ventricle $(11.1 \%)$, cerebellum $(22.2 \%)$, and suprasellar region (11.1\%). Histopathological examination revealed choroid plexus papilloma in 2 patients, pilocytic astrocytoma in 3 cases and ganglioglioma, neurocytoma, cystis dermoidalis or craniopharyngioma in the others. All patients were doing well after surgery and only one was re-operated because of the recurrence.

Conclusion: Incidentally found tumors are usually histologically benign. The prognosis for these patients is excellent. Taking into consideration long expected lifetime in pediatric patients surgical resection of the tumor seems to be an optimal option.

\section{FP051}

\section{Thalamic tumors - Approach and surgical results} Martin Smrčka (Brno, Czech Republic)

Introduction: Thalamic tumors are relatively rare tumors growing in a highly functional part of brain. They are more frequent in pediatric population. Their surgery is challenging and a high morbidity is possible. Relatively benign nature of many of these tumors means that an attempt for radical resection should frequently be performed. The approach has to be very carefully planned, sometimes with the help of modern diagnostic methods like DTI. The location and projection of the tumor in the thalamus plays an important role in choosing the approach. The pitfalls of these approaches are presented. Material: We have studied a group of 14 pediatric patients with thalamic tumors treated from 2005-2013. There were 10 males and 4 females, age ranged from 1-18 years (mean 11 years). Transcortical approach was used $5 \mathrm{x}$, transcallosal 5x, transsylvian $2 \mathrm{x}$ and supracerebellar infratentorial $2 \mathrm{x}$. 
Results: Gross total resection was achieved in 6 cases, subtotal in 5 and partial in 3 . There were 11 pilocytic astrocytomas, one subependymal giant cell astrocytoma, one diffuse astrocytoma G II and one glioblastoma. All patients are still alive with the mean follow-up 5 years. There was no permanent morbidity in this group.

Conclusion: Thalamic tumors might be safely radically resected if correct approach is used. The choice of approach is based in the projection of the tumor. Smaller tumors which are not close to the thalamic surface might be followed or biopsied if there is a likelihood of its malignant nature. Oncological treatment should be reserved for malignant tumors.

Acknowledgement: Supported by the Institutional support of University Hospital Brno.

\section{FP052}

Anti-tumor activity of amblyomin-X, a new tick Kunitztype inhibitor, in pediatric anaplastic ependymoma

Lorena Pavon, Tatiana Sibov, Silvia Toledo, Romero Cabral, Luciana Marti, Jackeline Malheiro, Alberto Tanus, Sergio Oliveira, Nasjla da Silva, Andreia Capelano, Ana Chudzinski, Fernando Paiva, Suzana Malheiros, Manuel Paiva-Neto, Sergio Cavalheiro (Sao Paolo, Brazil)

Introduction: Ependymoma is the third most common malignant brain tumor of childhood, accounting for $6 \%-12 \%$ of all intracranial tumors in children and up to $30 \%$ of brain tumors in those younger than 3 years. These tumors originate either from the wall of the cerebral ventricles or from the spinal canal. Despite gross total resection and radiation, $40 \%-60 \%$ of ependymomas recur at the primary tumor site or with metastatic spread, especially tumors in young children that show anaplastic features. Currently, the standard therapy for a low-grade ependymoma includes total surgical excision and may be followed by radiation therapy. Complete surgical resection is often not possible due to tumor location and concerns about damaging the surrounding eloquent brain during surgery. To develop more effective therapies for the treatment of ependymomas, improved understanding of action of the new therapeutic targets is urgently needed.

Objective: The aim of this study was to evaluate the antitumor activity of Amblyomin-X, a serine protease Kunitztype inhibitor in ependymoma primary culture from children and experimental model.

Methods: Cellular cytotoxic effects of Amblyomin-X were evaluated on normal human fibroblast (HDF-a) and ependymoma primary culture by MTT assay and flow cytometry using propidium iodide and FITC-conjugated Annexin-V and also by MRI in experimental model by stereotactic coordinates for intracerebroventricular infusion of ependymoma cells from a 1-10 year-old patient.

Results: Amblyomin-X was able to induce cell death in ependymoma cells, and, interestingly, the cytotoxic effects were not observed on human fibroblasts, which were used as a negative control. MRI analyzes demonstrated significant tumor mass regression when compared to untreated control.

Conclusion: Our results indicate that Amblyomin-X selectively acts on tumor cells by inducing apoptotic cell death. The present work opens perspectives for the characterization of new molecules involved in ependymoma control.

\section{FP053}

Neurosurgical management of orbital lesions in children in a developing country

Souad Bakhti, Nabila Tighilt, Hakim Kerrouche, Nazim Boumahdi, Youcef Benmouma, Mohamed Djennas (Algiers, Algeria)

Orbital lesions are sometimes managed by neurosurgeons. They can sometimes sight threatening. Patients can be either managed in ophthalmologic or neurosurgical department. Prognosis depends on histology but also but also management time. We report a retrospective study of 29 children with orbital lesions managed in our department during a period of 5 years. Age was varying from 2 months to 15 years. Proptosis was the main clinical sign in 25 patients with 6 patients presenting huge exophtalmia. Diminution of visual acuity was observed in 8 patients and 5 patients were admitted with blindness. These symptoms were mainly in relation with a delayed diagnosis. Transcranial approach was used in 17 patients while lateral approach while lateral approach was done in 11 cases. Exenteration was performed in 1 patient. Total resection was achieved in 15 cases some of them required a bony reconstruction which was done by autograft. Predominant lesions were rhabdomyosarcomas followed by dermoid cysts. Main complication was regressive ptosis and one patient deceased in post operative period. Recurrences occurred in 2 patients; both of them were reoperated. Multidisciplinary management when done was successful.

Surgery is the mainstay of treatment. Recovery depends on early diagnosis which is not always done in developing countries. Quality of resection depends intraoperative aspects such as adherences to the optic nerve or intraorbital muscles. Multidisciplinary management is mandatory for malignant lesions.

\section{FP054}

Use of 5-ALA fluorescence to guide resection of cerebral neuroepithelial tumors in children

Kirill Sysoev, Oleg Don, Alexander Kim, Constantine Samochernyh, William Khachatrian (St. Petersburg, Russia)

Objective: Evaluation of fluorescence-guided resection of pediatric cerebral neuroepithelial tumors.

Material and Methods: Twenty children aged from 3 to 18 were retrospectively analyzed in 2012-14, from the point of 
view of efficiency and safety of fluorescence-guided resection of neuroepithelial tumor. For 5-ALA administration the Russian licensed Alasens preparation was used, dissolved in $100 \mathrm{ml}$ water (20 mg per kg body weight). The solution was ingested 4 hours before the planned tumor resection. The Alasens preparation was used only in the absence of contraindications, with the consent from the parents and approval of the Neurosurgery Institute Committee on Ethics. All the patients underwent, pre- and post-operatively, a complete clinical and MRI examination.

Results: Fluorescence was absent in 5 cases (2-Gr. I, 1-Gr. II, 2-Gr. IV); and was negligible in 4 cases (2-Gr. I, 1-Gr. II, 1-Gr. IV). In the other 11 cases, the use of fluorescence guidance increased the reliability of verification of tumor tissue. Grosstotal or near-total tumor removal was achieved in $16(80 \%)$ out of 20 cases, with $90.1 \%$ in the group with efficient fluorescence. No clinically relevant side effects were recorded in the patients involved. One patient showed transitory postoperative blood ALT and AST increase.

Conclusion: The use of 5-ALA fluorescence guidance proved to be a safe diagnostic method in pediatric patients, which can improve intra-operative neuroepithelial tumor detection. This may increase of the totality of blastomatous tissue removal. Nonetheless, a data base is required for further studies of fluorescence guidance efficiency in patients of different age groups, with tumors of different grades.

\section{FP055}

Correlation of ADC map with histological grade in pediatric brain tumors

Aimilia Moraiti, Georgia Papaioannou, Spyros Sgouros (Athens, Greece)

Introduction: The purpose of this study was to establish the possible correlation between ADC values and histological grade of pediatric brain tumors.

Methods: The study included only patients with full MRI imaging with ADC sequences, treated between 2008-2016, in whom the original digital data were available. ADC maps were calculated. Average ADC signal intensity values were measured in the largest tumor area. The values were correlated with benign / malignant tumor histology type.

Results: 24 patients with age between 4 months and 16 years old (mean) were included. Histology typres were : 4 medulloblastomas, 5 pilocytic astrocytomas, 4 glioblastomas, 2 ependymomas, 2 germinomas, 5 diffuse astrocytomas grade II , 1 atypical teratoid rhabdoid tumor and 1 pineocytoma. Hence there were 11 low grade and 13 high grade tumors.

Average ADC signal intensity values were: low grade tumors $1659.5 \times 10^{-6} \mathrm{~mm}^{2} / \mathrm{sec}$ (range $1328-1981$ ), high grade tumors $1056.1 \times 10^{-6} \mathrm{~mm}^{2} / \mathrm{sec}$ (range: $722.9-1535$ ). There was statistically significant difference $(p<0.03$, chi-square)
Conclusion: There is a strong correlation between average ADC signal intensity value and histological grade of the tumor. This may prove particularly useful in evaluating possible tumor recurrences.

\section{FP056}

The role of sex phenotype in pediatric CNS tumor incidence and survival

Ryan Mathew, Josie Hayes, Melpo Kapetanstrataki, Sarah Fleming, Roger Parslow, Susan Picton, Paul Chumas (Leeds, United Kingdom)

Introduction: Evidence exists, in germinomas and medulloblastomas, that patient sex significantly influences incidence and outcome -perhaps through differences in susceptibility to tumour genesis and response to treatment, between males and females. The role of sex phenotype in other paediatric CNS tumours is unclear.

Methods: Age-adjusted incidence and overall survival (OS) rates were collected from the Surveillance Epidemiology and End Result (SEER) registry (covering 28\% of US patients) between $2000-11$ for common paediatric ( $\leq 19$ years) CNS tumours - pilocytic astrocytoma (PA), anaplastic astrocytoma (AA), glioblastoma multiforme (GBM), medulloblastoma (MB), sPNET, ependymoma (EP), germinoma (G) and pleomorphic xanthoastrocytoma (PXA). Patients with histologically confirmed, ICD-03 coded, first tumours, were included. Mortality was defined as any cause of death between 1-60 months of diagnosis. Results were statistically analysed by Incidence Rate Ratio (IRR), Kaplan-Meier and Log Rank (Cox Proportional Regression Hazard) Testing ( $<<0.05$ ).

Results: Total cases: males $=3169$, females $=2543$. Higher incidence was observed in males for all tumours, except PA. Compared to the opposite sex at the same ages; 1 - and 5-year OS was better in - males aged 3-4 years for MB, sPNETs, GBM and AA, females aged 0-19 years for MB, females aged 0-2 years and 15-19 years for AA; 5-year OS was better in females aged $0-2$ years with EP; 1 -year OS was better in males aged 3-4 year with AA.

Conclusion: Interrogating population-based registries such as SEER minimises bias and provides credible data. The differences in incidence and OS between the sexes for different paediatric CNS tumours provide useful prognostic information for clinicians. Further investigation of possible epigenetic and hormonal differences may provide vulnerabilities unique to males or females that may be exploitable for therapy.

\section{FP057}

Surgery for intraventricular tumors in children $\underline{\text { Jorge W.J. Bizzi, André Bedin (Porto Allegre, Brazil) }}$

Introduction: Tumors of lateral ventricles (LV) and third ventricle (TV) comprises a heterogeneous group of lesions in 
children. These lesions can be located deep in the brain, as tumors in the pineal region and third ventricle, very bloody, in close proximity to very important structures, making a complete surgical resection challenging. The purpose was to review the surgical results.

Methods: 374 patients, under 18 years of age, received surgical treatment for intracranial tumor and 42 (11\%) were located in the lateral ventricles and third ventricle. Data was collected prospectively, and reviewed from our brain tumor database. Tumors from selar and paraselar region invading the anterior part of third ventricle, was excluded from this review. Results: Were operated 22 boys and 20 girls. Age range from 1 month to 17 years, with an average of 6 years. The most frequent presentation was intracranial hypertension in 36 patients $(86 \%)$, with hydrochepalus in $34(81 \%)$. The tumor location was the LV frontal horn in $9 \%(n=4)$, trigono of the LV in $24 \%(n=10)$, LV occipital horn in $16 \%(n=7)$, TV in $19 \%$ $(n=8)$ and $31 \%(n=13)$ in the pineal region and posterior part of TV. The histopathology revealed choroid plexus tumor in $33 \%(n=14)$, being 9/14 carcinoma and 5/14 papilloma; astrocytic tumors in 10 (24\%), being 3/10 SEGA, germ cell tumors in $9 \%(n=4)$; ependymal cells in $7 \%(n=3)$; embrional tumors in $5 \%(n=2)$. Total resection was obtained in $52 \%(n=22)$, partial resection in $28 \%(n=12)$ and biopsy in $9 \%(n=4)$. Definitive treatment for hydrocephalus after tumor removal was necessary in 38\% (13/34) of patients with hydrocephalus. There was no transoperative mortality or surgical mortality in 30 days.

Conclusion: Although access lesions in the LV and TV can be difficult, a successful surgery can be done in the majority of cases with a low mortality.

\section{FP058}

\section{Meningeoma in children: report of 11 cases}

Jakob Nemir, Hrvoje Jednačak, Maja Jurin, Danijela Kolenc, Krešimir Saša Duric, David Ozretić, Pavle Miklić (Zagreb, Croatia)

Aim: Meningeomas in pediatric population are extremely rare. The aim of this study is to report on the manegement and the outcome of a series of eleven patients of pediatric meningeomas that were treated in our institution.

Methods: All pediatric patients with intracranial meningeomas operated on in period from january 1998 to june 2014. in Department of neurosurgery of Medical faculty in Zagreb (DNMFZ) were included. Preoperative planning was based on structural and when necessary functional MR. All surgeries were performed microsurgicaly, with neuronavigation and intraoperative EP monitoring when necessary. The study is based on retrospective analysis of continuosly updated datebase that includes patient history, radiological files, operative and pathohistological reports and follow up. Factors included in investigations were age, gender, tumor location and histology, extend of surgery, type and rate of complications, adjuvant treatment and progressioin free and overal survival.

Results: Eleven (11) children with intracranial tumours were operated on in the exammined period in DNFZ. The youngest was 4 the oldest 16 years old with a male predominance $(8 \mathrm{M}$ and $3 \mathrm{~F}$ ). There was one patient with neurofibromatosis. The simptoms and signs on onest were tumor location depended. The surgical resection was total in all patients (Simpson I and II). After histological review 7 patients were WHO grade I, 4 patient were WHO II ( 2 were clear-cell meningeomas grade II). 10 tumors were localized supratentorial while one was located in posterior fossa. Median follow up was 3 years. Two patients had local recurrence and were reoperated within 2 and 3 years. There were no postoperative mortality and the rate of morbidity and complications was low. In the three patients presented with epilepsy one is seizure free and two with goood seizure control.

Conclusion: Meningeomas represent less then 2-3\% of all pediatric intracranial tumors. Extent of surgical resection and patohistology are most important prognostic factors. There is need for larger and prospectice studies to investigate the biological behavior of meningeomas in pediatric population.

\section{FP059}

\section{Spinal cord tumors in children: a review of 21 cases}

Isabelle Merlot, Julie Francois, Jean-Claude Marchal, Anthony Joud, Pascal Chastagner, Olivier Klein (Nancy, France)

Introduction: Spinal cord tumors of children (SCTC) are rare with a frequent diagnosis delay. Their management is multidisciplinary and challenging due to their functional implications. The position of surgery is now better established, but the role and timing of chemotherapy (CT) and radiotherapy (RT) is still under debate. Adverse effects of treatments are important to take into account in the follow up of these children.

Material and Methods: 21 SCTC were referred to our institution from 1990 to 2014. Data regarding age, sex, diagnosis delay, clinical examination, MRI, surgery, pathology, other treatments (CT and RT), orthopaedics issues and follow up of these children were retrospectively recorded.

Results: Mean age is 8 years (4 months-17 years). Mean diagnosis delay is 5.5 months. All children (10 girls, 11 boys) were operated on (10 partial removals, 7 subtotal and 4 gross total removals) as a first line treatment. Pathological results showed 12 juvenile pilocytic astrocytomas, 1 grade III astrocytoma, 1 grade IV astrocytoma, 3 oligodendrogliomas, 2 ependymoma, one glioblastoma and one rhabdoid tumor. Fourteen children $(66.7 \%)$ received additional treatment: 12 CT and 7 RT. Ten children suffered from post-operative spinal deformities. Mean follow up is 71 months (5 months-180 
months), and, at this deadline are noted 8 tumor progressions and 4 deaths.

Conclusion: Surgery is the goal standard for SCTC and the only appropriate treatment in case of low grade lesion with stable disease on MR follow up. Additional treatments must be kept for high grade lesions or tumor progressions not attainable by a second look surgery. Spinal deformities are a frequent complication. Overall survival and event free survival mostly depends on pathology. Studies involving more centres are mandatory with the aim of collecting more cases and draw firmer conclusions about management of these tumors.

\section{FP060}

Spinal cord tumors in children and adolescents: diagnosis and treatment

Georgiy A. Kevorkov, Tetyana A. Malysheva (Kiev, Ukraine)

The diagnosis and treatment of the spinal cord tumors and spine tumors in children and adolescents is an actual medical and social problem. The polymorphism of clinical implications in children leads to late recourse. When neurosurgical stage is selected, the number of factors are integrative estimated, that bases the amount of neurosurgical treatment.

Purpose: To establish the features of spinal cord tumors in children to determine the appropriate treatment strategy.

Materials and Methods: Analysis of clinical-neurological, instrumental, morphological observations of 57 spinal cord tumors in children. CAT, MRI, and radioisotope scanning are the most informative methods.

Results: The most frequent primary symptom of the disease in children is the local pain, in which is not paid enough attention. In the later stages "fixed" position of the body, hyper tonus of back muscles, symptoms of spinal cord lesions. The tumors of the cervical spine, including craniospinal, are manifested primary by symptoms of the roots lesions of this level and by the development of motor and sensory disorders of conductive nature. The tumors of the spinal cord in children were diagnosed at the thoracic level in $43.6 \%$, at the high lumbal level -in $23.8 \%$, and at the cervical region- in $32.6 \%$.

Selection of the strategy depends on the location, the size and morphological characteristics of the tumor. Surgical interventions are aimed at removal of the tumor as possible, by providing decompression. In the significant lesion of the spinal cord the biopsy of tumor was performed to determine of the tactics of combined treatment. At the widespread lesions of the spinal cord in children to prevent of scoliotic disorders the osteoplastic spondylotomy is expedient.

Morphological diagnosis was verified: astrocytomas (75.2\%), anaplastic astrocytomas (12.5\%), anaplastic ependymomas (9.8\%), mixopapillary ependymomas $(1.8 \%)$, paragangliomas $(0.7 \%)$.

Conclusions: The amount of removal of the tumor depends on the location, histobiology and the spreading of the process.
The $80-90 \%$ of tumors in children under one year were congenital. During invasive spreading in order to prevent the destructive changes in the spinal cord the partial removal of the tumor is justified for the morphological diagnosis and subsequent combination treatment.

\section{Session 6: Neurovascular management}

\section{FP061}

Management of pneumococcal meningitis in infants associated to acute intracranial pressure

Federico Di Rocco, B. Vanel, Alexandru Szathmari, M. Berthon, P. Landzberg, E. Javouhey, Carmine Mottolese (Lyon, France)

Differently from the most common bacterial meningitis, pneumococcal meningitis is often associated to a severely increased intracranial pressure (ICP) which may jeopardize the survival rate and result in long term sequelae.

We reviewed our experience with infants with severe pneumococcal meningitis and analysed retrospectively the management of raised ICP.

Patients and Methods: Charts of children admitted to the intensive care unit of the pediatric hospital of Lyon between 2010 and 2015 were reviewed. Inclusion criteria for this analysis were: pneumococcal meningitis and raised ICP confirmed by ICP monitoring.

Results: Between 2010 and 201520 children presented with a severe pneumococcal meningitis confirmed at bacteriological examination. Mean age was 26.3 months ( $\min 2, \max 100$ ). In 16 of them the ICP was monitored with an ICP monitoring because of a severe neurological status.

Despite of the medical treatment, 7 of them, required a CSF diversion for ICP control.

An external ventricular drain was placed in 4 children with acute hydrocephalus at CT scan. One child had a subdural collection that was managed with an external drain. Finally, in further two children, an external lumboperitoneal drain was placed because of raised ICP refractory to medical therapy and slit ventricles.

The infection was cured in 17 children with 10 of them presenting moderate to severe neurological disability. Three children died.

Discussion: Severe pneumococcal meningitis may be associated with an acute or subacute increase in ICP. The recognition of such a raised ICP is of paramount importance for the correct the management of this severe pathological condition in order to contribute to the proper medical treatment. In our experience extrathecal CSF diversion was required to counteract the abnormally high increased ICP in the acute phase and to monitor the effect of the medical treatment later on. Unfortunately, the surgical management in pneumococcal meningitis seems to be not such effective like it occurs in bacterial meningitis of 
other etiologies. This study demonstrates the high mortality rate and the high incidence of severe long term sequale in survivors consequently emphasizing the importance of the specific prophylactic vaccination.

\section{FP062}

Pediatrics brain arterio-venous malformations (AVM): presentation, treatment modalities and outcomes

Anan Shtaya, Owen Sparrow (Southampton, United Kingdom)

Introduction: AVM treatment aims at achieving angiographic cure with no or minimal risk through surgical excision, endovascular embolization, radiosurgical lesion obliteration, or often by a combination of these modalities.

Methods: We studied all cases in the prospectively maintained vascular data-base of the senior author. We included all patients referred to our neurosurgical centre from 19942014. Of 48 patients aged $<18$ years, 43 had brain AVMs, 26 males and 17 females, with mean age of $12.6 \pm 0.6$ (range 4-17 years).

Results: Thirty-five patients had bled, 7 patients were symptomatic without hemorrhage and 1 was incidental. SpetzlerMartin score distribution was: 4 cases grade I, 16 grade II, 21 grade III, and 2 cases were grade IV. Appropriate imaging was performed, CT angiogram/MRI (in emergency cases) or cerebral angiogram, prior to definitive treatment. There were 37 supratentorial and 6 infratentorial AVMs. Twenty-seven patients had microsurgery alone and 9 patients were treated by radiosurgery only. Three patients were embolized, all followed by radiosurgery, with one requiring surgery too, while 4 patients had combined surgery and radiosurgery. Good outcomes, classified as modified Rankin score (mRS) 0-2 improved significantly after intervention to $90.6 \%$ from $37 \%$ pre-treatment ( $\mathrm{p}$-value $<0.0001$ ). Imaging confirmed obliteration in 27 cases from the surgery only group, while 7 patients who had radiosurgery only were cured. The rest underwent more than one treatment modality before achieving cure. We noted that para-trigonal AVMs with intraventricular hemorrhage were difficult to treat, often necessitating more than one modality of treatment. Cerebral angiogram at age 18 was negative in all patients who had been deemed cured previously.

Conclusions: Microsurgical excision of intracranial AVMs which are surgically accessible remains the primary treatment option with very good outcomes. AVMs were acutely removed when associated with a large clot that required evacuation, otherwise definitive management was performed on an elective basis.

\section{FP063}

Considerations in decision-making in the management of pediatric AVMs
Ibrahim Jalloh, Riki Trivedi, Ramez Kirollos (Cambridge, United Kingdom)

Introduction: AVMs in children are more likely to present with haemorrhage and have a greater lifetime haemorrhage risk compared to AVMs presenting in adulthood. Hence, although the natural history is not completely understood, intervention with the aim of obliteration is recommended for most paediatric AVMs. Microsurgical resection at the time of clot evacuation potentially reduces the overall burden of treatment risk.

Methods: An analysis of data collected prospectively from consecutive paediatric patients who underwent microsurgical AVM resection at a single UK neurosurgical unit between 2002 and 2015.

Results: 22 children (10F; 12M), from 3-18 years of age, underwent microsurgical resection. 15 had Spetzler-Martin grade 1 or 2 AVMs and 7 had S-M grade 3 or 4 AVMs. 17/ $22(77 \%)$ presented with haemorrhage 4 of whom were in coma (GCS $\leq 8$ ) including one with fixed and dilated pupils. 7 underwent early resection within 48 hours of presentation with haemorrhage. 5 children had microsurgery for an unruptured AVM. 1 patient underwent embolisation prior to resection and 2 patients underwent adjuvant stereotactic radiosurgery. 20 (91\%) had a good outcome (mRS 0 or 1). 2 AVMs recurred requiring retreatment. There were no significant differences in outcome between AVMs treated within $48 \mathrm{hr}$ and those treated later although one child with a large cerebellar AVM treated early had a diffuse residual nidus on postoperative angiography.

Conclusions: Microsurgical resection of AVMs in children produces good clinical outcomes and obliteration rates when managed by an experienced vascular surgeon even when performed in the acute setting at the time of haematoma evacuation. Pitfalls of early resection include distortion of AVM anatomy with risk of incomplete resection. Angiographic follow-up of AVMs is recommended due to risk of recurrence in children.

\section{Session 7: Craniofacial surgery}

\section{FP064}

Antenatal diagnosis of craniosynostosis: the necessity of pluridisciplinary consultation for the correct counseling Federico Di Rocco, Alexandru Szathmari, Massimiliano Rossi, Christian Paulus, Marie Pierre Cordier, Laurent Guibaud, Corinne Collet, Carmine Mottolese (Lyon, France)

In last years an increasing number of craniosynostosis recognized during prenatal life were reported demonstrating the improvement in prenatal ultrasound recognition of such malformations. 
In several instances the most easy to be recognized are the complex synostosis associated to typical anomalies of the face or limbs.

It is of paramount importance to individuate already in prenatal life not only the presence of complex craniosynostosis but also to define its type to provide a correct prenatal counseling. In this report we describe the difficulties encountered in the specific diagnosis of a complex synostosis initially referred as trigonocephaly and successively on repeated ultrasound examination recognized to be a complex craniosynostosis because of hexadactily of the 4 extremities and visceral malformations.

A suspicion of Carpenter syndrome was raised suggesting carrying out afotal CT scan which confirmed the fusion of the metopic suture and the partial suture of other sutures related to the bregma.The family could be properly informed.

Post-natal examination confirmed the complex synostosis involving the metopic, sagittal and coronal sutures. No hydrocephalus was present at brain imaging. Moreover an abdominal hernia and ectopic testis were found.

The diagnosis of Carpenter syndrome was confirmed by molecular analysis showing a RAB23 homozygote p.Tyr79* mutation.

The child was operated on for his complex synostosis without complications.

In conclusion, such observation of a Carpenter syndrome with a complete fusion of the metopic suture and only partial of the coronal and sagittal sutures underlines the broad spectrum of craniofacial abnormalities found in such a syndrome. It highlights the difficulties of antenatal diagnosis and correct parental counseling. This case also demonstrates the necessity of a specialized consultation possibly done in a referral center in case of suspected craniosynostosis even when the initial diagnosis appears to be a simple form.

\section{FP065}

\section{Scaphocephaly in children over 1 year of age}

Federico Di Rocco, Alexandru Szathmari, Nicolas Pianton, Christophe Rousselle, Isabelle Sabatier, Carmine Mottolese (Lyon, France)

Background: The early fusion of sagittal suture is the most common type of synostosis. Often recognizable at birth or in the first months of life, it is surgically corrected during the first year in nearly all the cases. However, in some instances the diagnosis is missed or excessively delayed resulting in a late repair. Though, surgical indication and management after one year of age are still debated in the literature, no papers have addressed the actual incidence of the phenomenon and its possible consequences such as the neurological condition and the impact on the surgical technique in cases of surgical management.
Patients: Charts of children with scaphocephaly operated on after 1 year of age at the Pediatric Neurosurgery Department of Lyon between 2006 and 2015 were reviewed. Exclusion criteria were: parents refusing the surgical operation or surgical indication was discarded by the surgeon.

Nineteen children that had undergone late surgical repair for the study out of 96 scaphocephalic subjects were considered. The mean age of the 19 children at surgery was 32.5 months (range 13 to 67 months ) whereas the mean age at referral was 25.2 months at referral (range: 0.5 to 63 months). The gender distribution was 3 girls and 16 boys.

Results: The skull deformation had been recognized at birth in 9 children. Three of them had their surgery delayed because of a poor medical condition in the first months of life (mean age at referral was 6 month and at surgery 18 months). In further 5 cases, the malformation, though correctly identified, was neglected by the parents until they decided to ask for further consultation at a mean age of 30.8 months. The remaining child had been operated on elsewhere for a scaphocephaly at 5 months of age but he needed a second surgery at 5 years of age following the recognition of a papilledema. None of the other 8 children presented papilledema or signs of raised intracranial pressure.

In a second group of 10 children the deformation had not been recognized by the family doctor until a new practitioner took care of the child ( 6 children, mean age 30.8 months). In the remaining 4 cases the synostosis was actually diagnosed only after neuroimaging studies performed for an aspecific psychomotor retardation (mean age: 41.7 months). None of these children had developed overt signs of raised intracranial pressure or papilledema during the life.

All the children underwent a surgical remodeling with parietal enlargement and sagittal sinus decompression.

Discussion: About a fifth of the children requiring surgical treatment received a delayed treatment at Pediatric Neurosurgical Unit of Lyon. This fact demonstrates an under-evaluation or missed recognition of this malformation among the obstetricians and the pediatricians in this series as well as in other series reported in the literature. By excepting cases which are operated late after having recovered from critical condition, late surgical operation is in most cases carried out only when the secondary cranial deformation becomes obvious. In other subjects, recognized to harbour the malformation after neuroimaging studies originally meant at investigating the possible causes of psychomotor retardation, the surgical repair is requested in the hope to improve the psychomotor delay.

This study further demonstrates that children receiving excessively delayed treatment are not so rare. Consequently, a multiple centers investigation could be advocated to detect the causes accounting for the late referral, the possible neurological and cognitive impact of the long persisting sagittal synostosis and, finally, the possible beneficial effect of the surgical repair even when performed lately. 


\section{FP066}

Human foramen magnum area and posterior cranial fossa volume growth in relation to cranial base synchrondrosis closure in the course of child development

Guillaume Coll, Emmanuel De Schlichting, Federico Di Rocco, Isabelle Barthelemy, Jean Marc Garcier, Jean Jacques Lemaire, Laurent Sakka (Clermont Ferrand, France; Lyon, France)

Purpose: We performed a retrospective study to assess (i) the variation of the foramen magnum area (FMA), (ii) the posterior cranial fossa volume (PCFV), depending on cranial base synchondroses ossification during childhood.

Patients and Methods: Madeline and Elster's scale was used to analyze the ossification of 12 synchondroses on millimetric computed scan slices from 235 children (103 girls, 132 boys). FMA and PCFV were measured on Iplan Stereotaxy 3.0.2.

Results: Mean FMA was higher $(p<0.001)$ in boys $(7.67$ $\left.\mathrm{cm}^{2}\right)$ than in girls $\left(6.49 \mathrm{~cm}^{2}\right)$. We observed a biphasic growth in both sex, a rapid phase from birth to 3.75 years-old then followed by a phase of stabilization. Comparing males to females, first phase was longer (ending at 4.33 yo vs 2.60 yo) with a lower rate. PCFV was higher in male $(\mathrm{p}<0.001)$ and followed a two phase process in both sexe, a rapid phase from birth to 3.58 yo, then a phase of slow growth until 16 yo. In male, rapid growth phase was longer than in female (from birth to 4.5 yo vs birth to 2.67 yo) and with a lower rate. Regarding the pattern of synchondroses closure, posterior interoccipital synchondroses close first, followed by anterior interoccipital (AIOS) and occipitomastoidal synchondroses, and lamdoids sutures simultaneously, then the petro-occipital and spheno-occipital synchondroses in the same time.

Conclusion: We found a pattern in the synchondrose closure. At birth, FMA and PCFV are constitutionally higher in boy than in girls $(\mathrm{p} \leq 0.024)$ and sex related difference in FMA is due to earlier closure of AIOS in girls $(p=0.007)$.

\section{FP067}

\section{Bicoronal synostosis: the role of fronto-orbital} advancement

Matthieu Vinchon, Pierre Guerreschi, Alexis Wolber, Irene Stella, Patrick Dhellemmes, Philippe Pellerin (Lille, France)

The optimal management of bicoronal synostosis (BCS) is controversial; posterior calvarial expansion has been advocated as the first-line operation. In our department, we have developed a technique of anterior frontoorbital advancement (FOA) with kyphosis of the bandeau, allowing important volume increase and simultaneous craniofacial distraction (CFD) if indicated.
We reviewed our experience with BCS, syndromic or not, during the past two decades. When indicated, faciostenosis was managed using craniofacial distraction osteogenesis, without facial osteotomy.

We reviewed 92 observations of BCS, 60 of these $(65 \%)$ syndromic, operated since 1994. 23 young infants (mean age 2.7 months) underwent perifrontal craniectomy; 69 (mean age 31.8 months) underwent FOA, 18 of these with CFD during the same session. Three patients had undergone posterior craniectomy previously. Ten patients required repeated craniotomy for FOA, 5 of these with CFD. Overall, CFD was performed in 23 patients. 65 patients $(71 \%)$ underwent one craniotomy for BCS, 25 patients underwent 2 , and 2 patients underwent 3 craniotomies. Mean head circumference and cephalic index improved from $1.26 \mathrm{SD}$ and 90.7 preoperatively to $-0.33 \mathrm{SD}$ and 83.3 respectively at last control.

The mean follow-up was 86.7 months. Five patients, four with Pfeiffer syndrome and one with Crouzon syndrome and a peripheral neuropathy, died. At last control, 9 patients $(10 \%)$ had decreased vision, and 20 (22\%) had developmental problems. Schooling was normal in $50(70 \%)$.

Fronto-orbital advancement with kyphosis of the frontal bandeau is efficient as a first-line, one-step operation, allowing calvarial augmentation, correction of hypertelorism, and, when indicated, craniofacial distraction.

\section{FP068}

Evaluation of the quality of life in $\mathbf{2 5}$ patients operated on for unicoronal synostosis in two craniofacial surgery units (Toulouse and Lille)

Benjamin Plas, Sergio Boetto, Matthieu Vinchon, Jacques Saboye, Jean Christophe Sol, Franck Emmanuel Roux (Toulouse, France; Lille, France)

Unicoronal synostosis is the third form of non syndromic craniosynostosis and it affects $1 / 10000$ births. Despite early surgical treatment, facial deformation persists in most cases, is quite pronounced, and is accentuated by the fact that it is asymmetric. This facial asymmetry could have a negative impact on quality of life. To test this hypothesis, we evaluated the quality of life in children and adolescents who underwent unicoronal synostosis surgery in two craniofacial surgery reference hospitals (University Hospital of Toulouse and University Hospital of Lille).

All individuals in this study had a minimum age of 7 and were previously seen in consultation in order to have photographs taken and complete a quality of life survey. Two surveys were utilized according to age: one version for children aged 7 to 10 , and one version for adolescents aged 11 and above. All patients underwent bilateral frontoorbital advancement. Cosmetic evaluation was performed by a craniofacial surgeon through the extended Whitaker 
classification and a symmetry scale that takes into account frontal retrusion, vertical dystopia, facial scoliosis and temporal hollowing.

Twenty five patients were seen in consultation and evaluated for aesthetics and quality of life. Children and adolescents exhibited average quality of life scores and normal self-image as compared to the general population. However, in cases of lower aesthetic scores (especially asymmetry), analysis of overall quality of life was significantly lower.

Children and adolescents who undergo unicoronal synostosis surgery have a normal quality of life, but it appears that patients with decreased facial symmetry have a lower quality of life. We therefore encourage the attainment of the most accurate facial symmetry possible.

\section{FP069}

Developmental attainment in metopic craniosynostosis Maggie Bellew, Paul Chumas (Leeds, United Kingdom)

Introduction: It has been suggested that Metopic Craniosynostosis is associated with a higher rate of developmental delay than other types of non-syndromic craniosynostosis. Cognitive and behavioral abnormalities have been reported in the literature in about a third of patients with Metopic Craniosynostosis. The aim of the current study was to verify this theory.

Method: Developmental data were collated from patients referred to the Leeds Craniofacial Unit between January 1996 and January 2016. The data were examined to determine how these children fared across time in terms of their developmental attainment, the impact that surgery had on this and how they compared with patients with other diagnoses at long-term follow-up.

Results: The data suggested that these patients are indeed at greater risk of developmental delay than other types of Craniosynostosis. This was consistent across a range of measures, and effects became more apparent at long-term follow-up. Exposure to sodium valproate represented a greater risk of developmental delay. Surgery was also shown to have an impact.

Conclusions: The study confirmed the assertion that patients with metopic Craniosynostosis are at risk of developmental delay. This is important information for Craniofacial teams to have when caring for patients presenting with this condition.

\section{FP070}

A proposal for a new classification of complications in craniosynostosis surgery: the Leeds experience

Dmitri Shastin, Velu Guruswamy, David Bonthron, Maggie Bellew, Sharron Peacock, Vernon Long, John Goodden, Ian Smith, Lachlan Carter, John Russell, Mark Liddington, Paul Chumas (Leeds, United Kingdom)
Introduction: The advent of craniosynostosis surgery has been heralded by increasing number of operative techniques, producing debate as to the superiority of some approaches over others. While evaluation of cosmetic and neurodevelopmental results is inconsistent, complications have been used extensively for audit. However, these tend to be descriptive and non-standardised, making comparison difficult. Here we propose a new classification to facilitate assessment of outcomes.

Methods: All patients undergoing surgery for non-syndromic craniosynostosis in Leeds between 2010-2015 were included. Routine diagnostic, operative and recovery protocols were employed. Patients received regular reviews by surgical team and craniofacial nurse specialist throughout their stay, with wound check-up within two weeks of discharge and routine follow up appointments afterwards. Any adverse events were recorded prospectively and graded as follows: (0) no complications; (1) inpatient complication; (2) complications after discharge not requiring re-admission; (3) complications requiring re-admission; (4) long-term neurological deficit; (5) mortality. These were further subdivided based on time since original procedure, and length of stay.

Results: Over the period of five years, 107 procedures were undertaken in 104 patients. Demographics, craniosynostosis type, operative technique, details of surgery (including surgical time, rate of transfusion, anaesthetic parameters, and other) are presented. Complications are tabulated into respective categories and analysed.

Conclusion: Complications in craniosynostosis surgery appear to be underestimated, possibly owing to a retrospective nature of data collection in many studies. We report our morbidity, and propose a new classification which is reproducible, easily utilised, avoids being specific to suture or procedure type, and could be extended to other aspects of paediatric neurosurgery.

\section{FP071}

Craniofacial distraction osteogenesis with rigid distractors for the treatment of syndromic craniosynostosis: a longterm follow up in a single center

Ricardo Santos de Oliveira, Andre Ando, Paulo Barreiro Marques-Neto, Marcelo Volpon Santos, Francisco Verissimo Melo-Filho, Antonio Augusto Velasco e Cruz, Helio Rubens Machado (Ribeirao Preto, Brazil)

Introduction: Frontofacial advancement by distraction osteogenesis (DO) is known to produce significant functional and aesthetic gains in the short and medium term. Little is known about the long-term effects of this technique. The authors present a long-term review of frontofacial advancement by distraction in patients with syndromic craniosynostosis. 
Objective: A critical evaluation of the use of DO in the surgical treatment of syndromic craniosynostoses in a single institution.

Methods: A retrospective review of the clinical data of 25 patients operated from 2003 to 2015 . The average latency period before activation of the rigid distractor was 5 days. Activation period ranged from 7 to 15 days, and the consolidation period was 8 to 12 weeks.

Results: All patients underwent craniofacial distraction with rigid distractors (19 monobloc fronto-facial, and six cases a Le Fort III midface advancement). The device was activated on an average of $15 \mathrm{~mm}$ (range, 7-22 mm). All patients had exorbitism, and 20 out of 25 cases $(80 \%)$ presented with chronic raised intracranial pressure pre-operatively, whilst 21 out of 25 (84\%) had severe upper airway obstruction. Short and long-term CT data demonstrated excellent ossification at the osteotomy sites post-distraction. All patients improved with distractive surgery correcting exorbitism $(\mathrm{p}<0.05)$, respiratory embarrassment $(\mathrm{p}<0.02)$, and stabilizing intracranial pressure. The midface was distracted an average of 16.4 $\mathrm{mm}$, with a range of 12 to $22 \mathrm{~mm}$. In two cases we observed a recurrence because the distraction device was removed too early due to complication. Cerebrospinal fluid leakage was the most common postoperative complication, occurring in 8 patients $(32 \%)$

Conclusions: Distraction osteogenesis has become a safe method for the correction of deformities of the craniofacial skeleton in children with syndromic craniosynostoses. It also involves a learning curve and a multidisciplinary team is mandatory.

\section{FP072}

\section{Risk for Chiari I malformation in untreated sagittal synostosis}

Laura Grazia Valentini, Veronica Saletti, Alessandra Erbetta, Luisa Chiapparini, Marika Furlanetto, Elena Beretta (Milan, Italy)

Chiari 1 Malformation (CM1) is a well known association with complex craniosynostosis, but it has been reported quite rarely with simple synostosis, as sagittal, both untreated and operated. We updated our series of Sagittal Synostosis untreated for misdiagnosis (USS) associated with CM1. In a monoistitutional series of 585 CM1 children clinical evidence of a cranial dolicocephaly was documented in 60 cases, in which a 3DCT scan was performed. It documented complete absence of the sagittal suture and indentation of all the other sutures in 32 cases and closed but previously patent sagittal suture in the remaining 28. All the patients were diagnosed in childage (4 to 15 years), except 4 young adults. All were submitted to genetic studies that ruled out a complex craniosynostosys. Three cases had severe mental retardation, 1 rapid visual deterioration due to increased ICP and 14 children had symptoms and 11 had evolving syrinx. They were submitted to Craniovertebral Decompression (CVD) with duroplasty, plus tonsils coagulation in 8; syringomyelia and symptoms solved by surgery. 1 child needed a shunt to treat associated hydrocephalus, because endoscopy before CVD was not enough to control ICP levels; another needed posterior cranial vault expansion to control intracranial hypertension. ICP levels were measured intra and postoperatively in 6 cases and were quite high until the duraplasty. The present series describes a new subgroup of Complex CM1, associated with USS. The pathogenesis of this CCM1 is due to both a completion for space and a deformation of the posterior fossa for a compensatory downward growth of the occipital bone. The recognition of USSCCM subtype is important, because the increased ICP levels may facilitate CSF collection and recurrence of tonsils descent.

\section{FP073}

Fronto-orbital remodeling in coronal synostosis treatment: review of a series investigating respective role of molecular diagnosis and surgery

Veronica Saletti, Francesca Di Pasquale, Fabio Mazzoleni, Luisa Chiapparini, Laura Grazia Valentini (Milan, Italy)

The aim of fronto-orbital advancement (FOA) in craniosynostosis is to prevent or treat functional problems and to obtain a better craniofacial shape in terms of symmetry and proportion, without interfering with normal growth. Evidence suggests that also molecular diagnosis can influence the surgical outcome.

With the aim to investigate the influence of surgical technique and genetic pattern on cosmetic results, we reviewed a sample of 33 children affected by coronal synostosis, unilateral in 26 and bilateral in 7 patients, surgically treated at FINCB. The age at surgery ranged from 5 to 26 months (average 8 months). All children were treated by the same type of cranioplasty: FOA with rigid plate fixation.

All children underwent molecular studies. FGFR3, FGFR2, FGFR1, TWIST1 and TCF12 genes were subsequently screened and a molecular diagnosis was obtained in one third of the series (11/33): the p.Pro250Arg mutation in FGFR3 gene was identified in 6 children, mutations in the TWIST1 and TCF12 genes were detected respectively in 2 and in 3 children. The pre and post-surgical evaluations included clinical assessment, photographic studies, CT images with 3D reconstructions and brain MRI. Cosmetic and functional surgical results were analysed in relation with molecular results A perfect correction, with complete craniofacial symmetry, was obtained in 23 cases; a less favourable result in 10 children, 5 harbouring the p.Pro250Arg mutation in FGFR3 gene and 1 TCF12 mutation. There were no major complications, neither lack of ossification or large deformities. 
The reported surgical technique allowed an accurate bone remodelling. The rigid fixation was effective for long term stabilization. Overcorrection of the defect partially compensated the recurrence due to the phenotype. Despite this, Muenke children had worse results.

\section{FP074}

Differential expression of candidate genes for human language-readiness in non-syndromic sagittal synostosis suggests shared molecular networks in craniofacial dysmorphology and cognitive evolution

Wanda Lattanzi, Daniela Chieffo, Luca Massimi, Lorena Di Pietro, Irene Bernardini, Marta Barba, Mirko Baranzini, Cedric Boeckx, Massimo Caldarelli, Antonio BenitezBurraco, Gianpiero Tamburrini (Rome, Italy)

Introduction: Non-syndromic sagittal craniosynostosis (NSC) is a congenital malformation, in which skull-brain landmarks are severely affected, as the skull is forced to acquire an elongated, less globular, shape. Cognitive impairment, typically involving the language domain, is reproducibly found in NSC patients. Interestingly, the skull of anatomically-modern humans (AMHs) shows a more rounded, globular shape compared to close extinct hominins. Differences in cognitive and linguistic abilities between species are also expected. This globular head shape is achieved during early postnatal life, where both skull and brain are in their critical window of plasticity. Recent studies point out that critical genes have been selected in AMHs, to play a key role in skull globularization, neurogenesis, neo-cortex and subcortical patterning, neuronal interconnection, synaptic plasticity, and cognitive function (including complex language).

We aimed to investigate the role of candidate genes for skull globularity and language-readiness in the premature ossification of calvarial sutures in NSC, chosen as a model disease.

Methods: Gene expression was analyzed, through real time PCR, in calvarial sutures of NSC patients, stratified based on language skills evaluation results. In vitro and in vivo assays were used to validate data.

Results: Differential expression of selected genes was found, including SFRP2 and SLIT2, among others. Some other differentially-expressed genes are predicted to be functionally linked to candidates for globularity and language-readiness. Functional assays confirmed a putative involvement of selected genes in premature suture fusion.

Conclusion: Taken together our data seem to suggest that the molecular signaling acting at the site of premature suture fusion in NSC overlaps with the gene regulatory network that shaped the skull and the brain during human evolution and that improved our cognitive abilities. We claim that this connection may be informative of the common molecular machinery regulating the skull-brain concerted development and evolution.

\section{FP075}

Analysis of operation cases of fibrous dysplasia in children Tomoru Miwa, Yoshiaki Sakamoto, Ryota Tamura, Maya Kono, Kazuo Kishi, Kazunari Yoshida (Tokyo, Japan)

Introduction: Craniofacial Fibrous Dysplasia (FD) shows destruction and sclerosis of the bone, and derived from in all parts of a skull and face. If patients are asymptomatic, observation is selected because the progress stops past the age of puberty. On the other hand, operation is selected in progress and symptomatic FD cases with their growth.

Methods: The characteristics and surgical roles of pediatric craniofacial FD operation cases (when diagnosed less than 18 years old) in our institution after 2007 were analyzed.

Result: We had 6 cases (Male: Female $=3: 3$, age average 11.7 years old), and the one case was McCune-Albright syndrome. A lesion was a case of parietal bone, maxilla, ethmoid sinus each and 3 cases of wide area of craniofacial lesion. Basically, shaving was performed for facial bone, and skull lesions were shaved or changed to the artificial bone. It was one case that showed visual loss, and was improved by a decompression surgery. The ethmoid sinus case was removed subtotally and reconstructed with plastic and otolaryngological surgeon. The purpose of other 4 cases of operation was mainly cosmetic. A period from a diagnosis to the first time operation is 3 years (median), and 2 cases needed for multiple times of operation. Relation between serum alkaline phosphatase (ALP) and aggressiveness of FD was not clear. Although all cases shows good coarse now, there is a possibility that patients will need an operation once again in future.

Conclusion: There are two purposes of FD operation, cosmetic and preserve the function. It is often no problem by observation, but early operation is necessary in case of visual loss by optic nerve compression. In case of pediatrics, sometimes multiple times of operation are needed because the progress continue until the end of adolescence, so wide range shaving or removal should be taken into consideration according to their age or progression speed.

\section{FP076}

Role of mechanosensory proteins PC1/PC2 in suture formation and craniosynostosis

Marios S. Themistocleous, Maria Katsianou, Christina Piperi, Penelope Korkolopoulou, Aimilia Moraiti, George Sfakianos, Efthimia Basdra, Athanasios G. Papavassiliou (Athens, Greece)

Introduction: Pathogenesis of craniosynostosis involves a variety of cellular mechanisms and processes, mediated by a range of signaling pathways that regulate skull growth. In 
addition, mechanical stretching is known to modulate postnatal skeletal growth and development. Polycystin 1 (PC1) and 2 (PC2) are integral membrane glycoproteins proposed to play a significant role in this process. The aim of study was to investigate the implication of $\mathrm{PC} 1 / \mathrm{PC} 2$ in suture development and suture fusion.

Methods: Presence of $\mathrm{PC} 1$ and $\mathrm{PC} 2$ proteins was investigated in rat sagittal (SAG) sutures tissue sections during postnatal development at p1/p5/p15 days of Sprague Dawley rats. PC1/PC2 localization and expression levels were investigated in primary suture SAG cell populations and human craniosynostosis tissue samples by RT-PCR, PCR, Western Immunoblotting and Immunohistochemistry.

Results: Western Immunoblotting revealed a differential expression pattern for $\mathrm{PC} 1$ and $\mathrm{PC} 2$ in $\mathrm{SAG}$ sutures at p1/p5/p15 days. PC1/PC2 levels were elevated at postnatal day 5. Immunohistochemical analysis of PC1/PC2 showed a nuclear expression in fused sutures. In primary suture SAG cell cultures, $\mathrm{PC} 1$ and $\mathrm{PC} 2$ presence was associated with an elevated expression of the osteoblast marker RUNX2 and a lower expression of chondrocyte marker SOX-9. PC1 and PC2 expression in human craniosynostosis samples was detected in synostotic sutures.

Conclusion: The identification of $\mathrm{PC} 1 / \mathrm{PC} 2$ involvement in premature obliteration of sutures will be of primary importance for understanding the underlying pathophysiology of craniosynostosis. Furthermore, it will allow detection of specific molecular targets to complement current therapy and diagnostic schemes.

\section{FP077}

Intra-operative dural tears impact in craniofacial procedures: analysis of $\mathbf{3 2 0}$ consecutive cases

Santosh Mohan Rao Kanangi, Christian Duncan, David Richardson, Christopher Parks, Sasha Burn, Ben Robertson, Ajay Sinha (Liverpool, United Kingdom)

Objective: To calculate the incidence of dural tears in craniofacial surgical procedures realised between 2010 and 2015 and to evaluate their impact in the post-operative course and medium-term follow-up.

Methods: We retrospectively review 305 patients operated on between 2010 and 2015 at Alder Hey Hospital. We analyse a surgical series consist 320 procedures: $50(15.6 \%)$ in syndromic or with multi-suture craniosynostosis and 270 (84.4\%) in non-syndromic single-suture craniosynostosis NSSS; 16 were re-operations.

We assess the incidence of intraoperative dural tear considering the surgical operation notes: we reported all cases when a suture has been applied to repair a CSF leaking.
Results: Sixty-seven dural tears $(20.9 \%$ of all procedures) have been reported: 23 in syndromic patients (34.3\%) and 44 in NSSS cases $(65.7 \%)$.

We observed 46 dural tears in FOAR surgery $(68.7 \%), 12$ in the subtotal vault remodelling (17.9\%), 4 in the posterior vault remodelling $(6 \%), 3$ in total vault procedures $(4.4 \%)$ and 2 in the strip craniectomy $(3 \%)$.

A dural tear has been reported in 7 out of 16 re-operations $(43.7 \%)$.

In 55 patients ( $82 \%$ of all dural tears), no post-operative or long-term consequences have been detected.

In only 13 cases ( $4.1 \%$ of all procedures), we noticed surgical complications related to dural tear: 3 intra-operative bleeding; 1 requiring duroplasty and a subsequent surgical wash out for wound infection; 1 CSF leakage requiring lumbar drainage; 1 supra-orbital pus collection; 1 orbital pseudo-meningocele; 1 opened frontal sinus; 5 wound infection requiring surgical debridement (one with bone exposure and one with deep tissue infection).

Only 2 patients are on anti-epileptic treatment at 3 years follow-up.

Discussion: Intra-operative dural tears are not so rare complications in craniofacial surgery ( $20 \%$ of procedures), but in only $4 \%$ of cases are responsible of real post-operative complications.

\section{FP078}

Russian experience in endoscopic-assisted craniosynostosis surgery and postoperative helmet-therapy of nonsyndromic craniosynostosis in children

Albert Sufianov, Iurii Iakimov, Saidi Gaibov (Tiumen, Russia)

Introduction: The objective of this research is to present our own experience of the use of endoscopic-assisted craniosynostosis surgery and postoperative helmet-therapy methods in treatment of nonsyndromic craniosynostosis in children.

Methods: Within the period between January, 2012 and May, 2015 in Federal Centre of Neurosurgery (Tyumen, Russia) there were 134 pediatric patients (104 boys and 30 girls) at the age of 5.02 \pm 1.1 months old with nonsyndromic craniosynostosis, including cases of scaphocephaly $(\mathrm{n}=74)$, trigonocephaly $(\mathrm{n}=45)$ and plagiocephaly $(\mathrm{n}=15)$. All patients underwent endoscopic-assisted craniosynostosis surgery combined with postoperative helmet-therapy.

Results: After performing endoscopic-assisted craniosynostosis surgery and postoperative helmet-therapy there were no cases of fatality or deterioration in neurological status in the cohort group of children during the whole follow-up period. Postoperative helmet-therapy started as soon as possible after endoscopicassisted craniosynostosis surgery procedures and lasted in average $6.44 \pm 1.69$ months in cases of scaphocephaly, $6.53 \pm 1.18$ months in cases of trigonocephaly and $6.7 \pm 1.5$ in cases of trigonocephaly. Four patients ( $3 \%$ of the total amount) experienced 
recurrence of nonsyndromic craniosynostosis. One child with trigonocephaly had a leptomeningeal cyst in eight months after the endoscopic-assisted craniosynostosis surgery + postoperative helmet-therapy procedures, which required surgical intervention. Conclusion: Taking into account a small amount of recurrences and complications after endoscopic-assisted craniosynostosis surgery combined with postoperative helmet-therapy, we have come to the conclusion that the offered method can be considered safe and effective for treatment of the children with nonsyndromic craniosynostosis.

\section{FP079}

\section{Craniosynostosis - Sutures or plates?}

Frantisek Horn, Michal Kabat, Dana Dubravova, Eva Stefankova, Michal Petrik, Pavol Babal, Martin Smrek (Bratislava, Slovak Republic)

Backround: The aim of the surgical correction in children with craniosynostosis (CS) is to restore the head with good cosmetic results, and to respect protection of the brain and head growth. Anthropometry is an excellent non invasive method in the diagnostics and long term follow up in patients with CS.

Objective: To compare the use of absorbable plates (group A) with absorbable sutures (group B) in fixation of the bones at surgery.

Methods: A prospective study of 43 patients with non syndromic craniosynostosis operated in 2006-2013 by the same team and approach. In group A, 16 patients we used absorbable plates for the fixation of the bones. Fused sutures in this group: sagittal 3, metopic 4, bicoronal 2, more sutures fused 7 . The age at the surgery was in average 7 months (interval 3-24 months). In group B, 25 patients with absorbable sutures: sagittal 19 , metopic 3 , squamous 2 , more sutures fused 1 . The age in average 6 months (3-20 months). Anthropometry approach was described by Martin and Saller (1957). We used: head circumference, length, latitude, transversal head arch, frontal and basal breadth, face highness and cranial index. Antropomethry was done: at the first examination, before surgery, after surgery once a year. For the comparison we used the data at 3 years follow up exam (group A 3,08 and group B 2,9 years).

Results: By comparing the values of cranial index in group A and group $B$ before and after surgery the cranial index is significantly better in the group $B$, with suture fixation $(p=0$, 03). In group $A$ in 4 patients beside the cranial index also growth was after the surgery below 2 percentil or up to 98 percentil, in group B only in one patient the parameters are out of criteria.

Conclusion: It seems that the use of absorbable sutures in restoring the child head with craniosynostosis leads to better results in head growth than plates.

\section{FP080}

Craniosynostosis - Techniques and outcome

Dwarakanath Srinivas, Girish Rao, Dhaval Shukla, Somanna Sampath (Bangalore, India)

Introduction: Craniosynostosis is an eminently correctable problem though oft neglected in our country. In this article we discuss the experience at our institute regarding the surgical management of craniosynostosis.

Material and Methods: This retrospective study included 36 patients who underwent surgery over a period of 7 years. The patient's records were obtained from the medical records. The demographic profile, clinico-radiological features, various aspects of surgical management, outcome and long-term follow-up were analyzed.

Results: There were a total of 36 pts (9-sagittal suture, 18-coronal suture, 2-metopic suture, 7-multi-sutural including 1 case of Aperts and 2 of Crouzon syndrome) operated at our institute. All patients with Brachycephaly/ Plagiocephaly/ Syndromic craniosynostosis underwent fronto-orbital advancement. We used either titanium or bioresorbable plates. The average duration of surgery was $176 \mathrm{~min}$ and blood loss $268 \mathrm{ml}$. The average age of surgery was 9.8 months. 8 patients had dural tears that were repaired. There were no postoperative CSF leaks. One patient had delayed wound infection (after 1.6 years and was managed with antibiotics and dressings). There were no long-term complications. The average duration of follow up was 2.6 years).

Conclusion: In this paper we present our experience with craniosynostosis, review the surgical techniques used and present operative videos and review long term follow-up and outcome.

\section{FP081}

Late approach for non-syndromic craniosynostosis Analysis of pitfalls and complications

Alexandre Canheu, Marcio Lehmann, Antonio Dias Marcos, Luis Koury, Henrique Galles Marcos, Roberto Parente Neto, Tomas Monte Raso, Luis Penzo, Felipe Da Silva, Paulo Henrique Pires De Aguiar (Londrina, Brazil)

Introduction: The late approach for craniosynostosis is not advisable. However pediatric neurosurgeons face this situation after unsuccesful former surgeries, uninformed parents, and lack of attention from general neurosurgeons. One should be aware of this issue, in order to avoid the pitfalls of this complex surgery.

Methods: Six children were operated on at our public university hospital, between 2013 and 2015, ranging between three and seven years old at the time of the surgery. All surgeries were headed by the same experienced pediatric neurosurgeon. 
The first two cases were scaphocephalic boys, performed cranial vault remodeling. The third case a girl with anterior right plagiocephaly, already operated on, performed a unilateral frontal advancement. The fourth case a brachicephalic boy, performed supraorbital bar advancement, and new frontal design. The fifth case, boy with pansynostosis, operated on for a cranial vault remodeling. The sixth case brachicephalic girl, performed supraorbital bar advancement, and new frontal design.

Results: The early complications were two: intraoperative massive bleeding in the pansynostosis after decompression of the superior sagittal sinus. We interrupted the procedure and leave the frontal bar advancement behind. Bleeding was controlled using hemostatic agents. The second, a reoperation for brachicephalic boy whose bicoronal previous incision was linear and fibrous thus impairing the elasticity of the skin. The plastic surgeon performed a "Y" relaxing incision successful in reconnecting the borders. Late complications were also two. Skin breakage was seen in four cases. A minor complication treated with dressings. In one case, we've seen the skin breakdown by a titanium screw, surgically removed.

Conclusions: The late approach turns the bone thick, promotes opening of venous channels, and loses the skin resiliency, especially in the reoperations. This situation should encourage the setting of a pediatric craniofacial team in every medical center, giving the best option to each case, avoiding complications.

\section{FP082}

Safety of cranial vault remodeling in children with nonsyndromic craniosynostosis - Single institution experience Petr Vacek, David Štěpánek, Vladimír Přibáň (Plzen, Czech Republic)

Introduction: The main goal of surgical treatment of craniosynostosis is to correct physical abnormalities of the craniofacial skeleton. This management has shifted from craniectomy to total cranial vault remodeling, and more recently back to craniectomy due to popularization of minimally invasive endoscopic approaches. In our institution we prefer total cranial vault remodeling. Although this procedure is elective, it's associated with a range of complications.

Methods: We present a retrospective review of pediatric patients suffered from nonsyndromic craniosynostosis and treated between May 2009 and January 2014 by cranial vault remodeling. We focused on demographic data of the patients, intraoperative and postoperative complications and finally on final cosmetic outcome ranked two years after surgery.

Results: A total of 22 children in the age from 5 to 15 months underwent cranial vault remodeling as a nonsyndromic craniosynostosis correction. All patients received intraoperative blood transfusion. Besides two intraoperative durotomies (repaired primarily) we didn't have any intraoperative or postoperative complication in our group of patients. The mean length of stay in the hospital was 9,3 days. In all cases we registered significant improvements in the cranial index and in the head shape.

Conclusion: Our experience of using cranial vault remodeling as a treatment of nonsyndromic craniosynostosis shows this technique effective and safe.

\section{Session 8: Intraoperative neuromonitoring}

\section{FP083}

Clinical, imaging and immunhistochemical characteristics of focal cortical dysplasia type II extra-temporal epilepsies in children: analyses of an institutional case series

Friederike Knerlich-Lukoschus, Mary B. Connolly, Glenda Hendson, Paul Steinbok, Christopher Dunham (Kiel, Germany; Vancouver BC, Canada)

Introduction: Extra-temporal FCD Type II A/B is not completely understood in terms of clinical, imaging, biological, and neuropathological differences. Our aim was to analyze distinctions between these two formal entities addressing clinical, MRI, and immunohistochemical features in extratemporal epilepsies.

Material and Methods: Cases formerly classified as Palmini FCD Type II non-temporal epilepsies were identified through the prospectively maintained epilepsy database at $\mathrm{BCCH}$, Vancouver, Canada. Clinical data including age of seizure onset, age at surgery, seizure types and frequency, affected brain regions, and outcome were obtained for each patient. Pre-operative MRI was re-evaluated. H\&E tissue sections were re-evaluated by using the ILAE 2011 classification system and further immunostained for standard cellular markers (NeuN, Neurofilament, GFAP, CD68) and additional established markers of pathology in epilepsy resections, namely CD34 and alpha-B-crystallin (ABC).

Results: Seven non-temporal ILAE 2011 FCD Type II A and 7 FCD Type II B cases were included in the study. Clinically, FCD Type II A cases presented with an earlier age of epilepsy onset and better surgical outcome. Radiology distinguished FCD Type II A and B in that FCD Type II B presented more frequently with characteristic cortical alterations. NFP staining confirmed dysplastic cells in dyslaminated areas in FCD Type II A/B. White/gray matter junctions were focally blurred in FCD II B cases (H\&E/LFB staining). ABC highlighted glial cells in the white matter and subpial layer in both FCD II subtypes, but only in FCD II Type B were ABC positive balloon cells seen. Finally, distinct nonendothelial cellular CD34 staining was found exclusively in MRI-positive FCD Type II B specimens. 
Conclusion: Extra-temporal FCD Type II A and B exhibited clinical, MRI and immunohistochemical characteristics with especially CD34 emerging as potential surrogate marker for lesional FCD Type II B.

\section{FP084}

Safe use of neuromonitoring in intraoperative high field MRI environment

Oliver Bozinov, Sandra Dias, Marian Neidert, Johannes Sarnthein (Zurich, Switzerland)

Purpose: High field intraoperative MRI (ioMRI) is becoming increasingly available in neurosurgery centers, where it has to be combined with intraoperative neurophysiological monitoring (IONM). Subcutaneous IONM needle electrodes remain on the patient during ioMRI and may cause image distortions and burns. We therefore investigated the prevalence of complications in children.

Methods: All subcutaneous needle electrodes 2013-2015 that remained on the patient during intraoperative 3 Tesla ioMRI scans were prospectively documented. The needle electrodes consisted of diagmagnetic $\mathrm{Pt} / \mathrm{Ir}$ if placed on the scalp and of stainless steel otherwise.

Results: A total of 416 needles remained placed during ioMRI in 14 children aged 18 months to 17 years. Of the needles, 100 were on the scalp, 92 above the chest, and 224 below the chest. Cranial nerve activity was recorded in 8 patients. One patient suffered from a skin irritation at his shoulder. All other electrodes had no side effects.

Conclusions: We have corroborated the history of safe use for subcutaneous needle electrodes in ioMRI.

\section{FP085}

Long-term evaluation of intra-operative neuromonitoring (IONM)-assisted tethered cord surgery. A retrospective longitudinal observational study

Sebastiaan E. Dulfer, Gea Drost, Rob J.M. Groen, Eelco W. Hoving (Groningen, the Netherlands)

Introduction: The use of IONM in tethered cord surgery is common practice, and its feasibility and safety are established. Surgery in case of a tethered cord syndrome is performed to relief, but also to prevent secondary symptoms in the neurological, urological and orthopedic field. In order to evaluate the efficacy of the detethering and the long-term effects on TCS symptoms, a substantial period of time for follow-up (FU) is required. This study has been conducted to analyze this long-term outcome after IONMassisted tethered cord surgery.

Methods: A total of 60 patients who underwent surgical detethering with IONM during a 9 year period (19992008) were included. 35 patients with either lipomyelomeningocele and/or a split cord malformation were considered as high-risk. All patients were scored on neurological deficits (muscle weakness and sensory disturbances), urological deficits, orthopedic deficits (scoliosis), and pain at four moments in time; pre- and postoperative, and during FU after a mean of 4.6 and a mean of 11.2 years. Changes in TCS symptoms over time were evaluated.

Results: The long-term outcome comparing pre-operative with last FU scoring, shows improvement on neurological, urological and pain symptoms in $10(16.7 \%), 8$ $(13.3 \%)$ and $21(35 \%)$ patients respectively. Neurological, urological and pain deterioration was found in $8(13.3 \%), 5(8.3 \%)$ and $3(5.0 \%)$ patients respectively. Progressive scoliosis occurred in 11 patients (18.6\%; mean age 16.7 years), of which 8 $(72.7 \%)$ belonged to the high-risk group. Nine of these 11 patients showed late progression of scoliosis between the first and second FU.

Conclusion: A long-term lasting effect of relieve of symptoms of TCS was found in this series after surgical detethering with the use of IONM, except for scoliosis, that appeared to be progressive in 11 patients.

This finding might suggest that tethered cord surgery may not prevent progression of scoliosis in this group of patients.

\section{FP086}

Pediatric intraoperative neurophysiological monitoring in posterior fossa surgery

Franco Randi, Andrea Carai, Gionathan Amante, Silvia Cossu, Alessandro De Benedictis, Raffaella Messina, Alessandra Savioli, Angela Mastronuzzi, Carlo Efisio Marras (Rome, Italy)

Introduction: Intraoperative neurophysiological monitoring (IOM) has recently become a promising tool in neurosurgery. Experiences in children are scattered and the real contribution of this technique to extent of resection and post-operative neurological impairment prevention has not be fully determined yet.

We describe our experience in 33 consecutive posterior fossa surgical procedures (30 patients, 19 males and 11 females) in the last two years.

Methods: Standard monitoring setting included trans-cranial motor evoked potentials (tcMEP), somatosensory evoked potentials (SEP), continuous electromyography (EMG) and brainstem evoked potentials (BAEPs).

Relevant IOM information was recorded and correlated to tumor characteristics, intraoperative modifications of surgical strategy, post-operative neurological deficits.

Results: Spontaneous EMG activations were observed in $100 \%$ of brainstem lesion, in $80 \%$ of those infiltrating the floor of the IV ventricle and in less than $50 \%$ of cerebellar 
hemispheric ones. Brainstem infiltration was found to be highly predictive for transient EMG activation (94\% in infiltrating vs $37 \%$ in non-infiltrating lesions). Transient EMG activation was highly predictive of early post-operative neurological impairment ( $84 \%$ at 72 hours) but did not correlate with long term neurological deficits ( $35 \%$ at 3 months).

MEP modifications were only found in presence of brainstem infiltration and resulted in significant modification of surgical strategy. $75 \%$ of patients showing potential drops were found to suffer early motor impairment after surgery. SEP modifications were also rare $(12 \%)$ but correlated to early postoperative sensibility/motor deficit (75\%).

No significant variation of BAEPs was observed in our study. No specific IOM pattern was observed in the two patients with post-operative posterior fossa syndrome.

Conclusion: IOM showed a good negative predictive value in posterior fossa surgery in children.

\section{FP087}

\section{Epilepsy surgery in benign tumors in children}

Michal Tichý, Josef Dvořák, Pavel Kršek, Josef Zámečník, Vladimir Komárek, Martin Kynčl, Petr Libý (Prague, Czech Republic)

Introduction: Benign tumors represent the second most frequent reason for epilepsy surgery in children (after the cortical dysplasias). Tumors as DNET and gangliglioma mostly affect temporal lobes and are often asociated with cortical dysplasia in adjacent cortex. Despite reported high succes rates of surgical outcomes in these series, abour $20 \%$ of patients do not reach the complete postsurgical control of seizures. Therefore the use of more precise preoperative evaluation and adequete surgical techniques is mandatory.

Material and Methods: Among 200 pediatric resective epilepsy surgeries there were 35 children operated on benign tumors and chronic epilepsy at Motol Epilepsy Centre, Prague. The age varied from 2-18 years at the time of resection. All children undervent complex preoperative evaluation including high resolution MRI, video/EEG , neuropsychological investigation. In indicated cases functional neuroimaging data were performed (FDG-PET, SISCOM, fMRI, DTI, MR spectroscopy). In 3 patients long-term invasive monitoring with intracranial electrodes placement was necessary. In majority of the cases the operation was performed as "multimodality image-guided surgery" with wide use of neuroimaging data in the neuronavigation system. Intraoperative electrophysiology including monitoring of motor functions and awake craniotomy technique was performed in selected cases. Histopatology examination prooved ganglioglioma in 25 and DNET in 10 patients. Localisation of the tumor was temporal in 19 cases, extratemporal in 13 cases and multilobar in 3 .
Results: There was no mortality or long-time severe morbidity in our group of patients. The hemianopsy as expected deficit was postoperativelly present in 2 patients. In 3 patients with lesions localised at the dominant temporal lobe a transitory mnestic deficit was observed.

In a group of 21 patients with follow-up more then 2 years there are 20 seizure-free patients, 1 patient has unfavorable outcome with persistent seizures despite repeated surgery, 9 patients are completely seizure-free without AED medication.

Conclusions: Epilepsy surgery in benign tumors and refractory epilepsy is fully indicated despite very slow biological activity of the tumors. One of the major goals is to prevent cognitive deterioration of patients. We have reached $95 \%$ postsurgical seizure-free rates; a significant proportion of our subject could withdraw AED medication and experienced cognitive gains.

Funding: Supported by MZ ČR - RVO, University Hospital Motol, 00064203.

\section{FP088}

Intraoperative neurophysiological monitoring for the resection of brain tumors in pediatric patients: tips and tricks

Francesco Sala, Angela Coppola, Vincenzo Tramontano, Micol Babini, Giampietro Pinna (Verona, Italy)

Introduction: Intraoperative neurophysiology (ION) is the gold standard to map and monitor brain functions during supratentorial surgery in critical areas. While the principles of most ION techniques are the same for adults and children, the developing nervous system has peculiar characteristics in terms of anatomical and physiological maturation of afferent and efferent pathways within the brain and the spinal cord.

Materials and Methods: We reviewed current ION techniques to assist during the resection of brain tumors, focusing on the aspects peculiar to mapping and monitoring of sensorimotor functions in the pediatric population. Cortical and subcortical mappings as well as motor evoked potential (MEP) monitoring were addressed based on our experience and a review of the literature.

Results: The following aspects should be considered when performing ION during brain tumors surgery in children. 1) The motor cortex is displaced frontally in younger children. 2) The physiological maturation of cortical and subcortical motor pathways takes much longer than anatomical connections and stimulation parameters should be adjusted accordingly. 3) The so-called MEP short-train technique is more successful than Penfield's technique in eliciting motor responses in young children.

Conclusions: ION techniques can be successfully applied to the pediatric population as long as specific adjustments are adopted. 


\section{FP089}

Intraoperative neuromonitoring in children with lumbosacral myelomeningocele

Yusuf Izci, Cahit Kural, Özkan Tehli (Ankara, Turkey)

Introduction: Myelomeningocele is a common form of open spinal dysraphisms. Early surgical intervention is required in order to prevent the infection and to protect the functional neural structures of the children. Intraoperative neuromonitoring (IN) may be used in these patients for the determination of functional neural structures and for the protection of them. Our aim is to report our experience on the use of IN in children with lumbosacral myelomeningocele.

Method: Ten newborns underwent surgical treatment for lumbosacral myelomeningocele within the 3 days after the birth. Surgical intervention, including reconstruction of the neural placode, duraplasty and skin closure, was performed in all patients using IN under controlled muscle relaxation. Electrical nerve root, spinal cord and neural placode stimulations were performed using direct monopolar stimulator to check the functions of neural structures. In addition, distal spinal cord was stimulated with bipolar probe and responses from the lumbosacral roots and compound muscle action potentials (CMAPs) from the external anal sphincter (EAS), vastus lateralis, tibialis anterior and gastrocnemius muscles were recorded.

Results: CMAPs from the EAS were obtained in $80 \%$ $(8 / 10)$ of the patients. Electrical conduction from the neural placode was recorded in all patients and responses were obtained from the lumbosacral roots. These roots were not sectioned. All fibrous bands or neural structures without any electrical conduction were sectioned in order to release the spinal cord and neural placode.

Conclusion: We recommend early repair of lumbosacral myelomeningocele with resection of the non-functional neural and fibrous structures, reconstruction of the neural placode, and duraplasty. Identification of functional roots by IN contributes to the safety of surgical treatment. The presence electrical conduction through the neuroal placode suggest that appropriate closure of the placode may result in better neurological outcome.

\section{Session 9: Hydrocephalus}

\section{FP090}

Surgery for intracranial arachnoid cysts in children - A prospective long-term study

Katrin Rabiei, Mats Högfeldt, Doria-Medina Roberto, Tisell Magnus (Gothenburg, Sweden)

Purpose: Intracranial arachnoid cysts are cystic malformations found in both adults and children. While many are asymptomatic, some cause symptoms and warrant surgical treatment. In this prospective population-based study, we aimed to study the short- and long-term outcome after surgical intervention in children with arachnoid cysts referred to our centre.

Methods: Twenty-seven pediatric patients (13f, 14m, mean age 9.4 yrs) with de novo cysts were consecutively included during a five-year period. The presenting symptoms were headache $(n=12)$, balance disturbance and dizziness $(n=6)$, seizures $(\mathrm{n}=6)$, hydrocephalus ( $\mathrm{n}=5)$, and macrocephaly ( $\mathrm{n}$ $=1$ ). Twenty-two patients underwent surgical treatment with either microsurgical $(n=17)$ or endoscopic fenestration $(n=5)$ of the cyst wall. The cyst volume was measured with OsiriX software pre- and postoperatively. Short-term- and long-term follow-up of all patients was conducted three months and 8.6 years (7-10.5 yrs.) postoperatively.

Results: Three months after surgery, 59\% of the patients were improved regarding at least one major complaint and average cyst volume was reduced to $33.3 \mathrm{ml}(0-145$ $\mathrm{ml})$. At the long-term follow-up, $77 \%$ of the patients were improved. There was no permanent postoperative morbidity.

Conclusion: In $77 \%$ of the 22 patients who were operated on for arachnoid cysts as children, at least one symptom ceased after a mean follow-up of 8.6 years, but subjective symptoms remained in 13 patients (59\%). There was no permanent morbidity. We found no association between radiological reduction of cyst volume and clinical improvement.

\section{FP091}

\section{Long-term mortality rates in shunted hydrocephalic} patients

Matthias Gmeiner, Helga Wagner, Christoph Zacherl, Christian Auer, Willem J.R. van Ouwerkerk, Kurt Holl (Linz, Austria; Amsterdam, the Netherlands)

Introduction: Very long-term follow-up is rare for patients with hydrocephalus and shunt operation. The aim of this study was to determine the long-term mortality rates in these patients.

Methods: Pediatric patients with first shunt operation between 1982-1992 were included in this retrospective study. Patients with intracranial neoplasms were excluded. The time and cause of death were determined in each patient. Follow-up data were obtained and evaluated between 2013-2015.

Results: 145 patients were included in this study. Only one patient, who moved to another country at the age of 2 , was lost to follow-up and therefore excluded for statistical analysis. Etiology of hydrocephalus was intraventricular hemorrhage (31.3\%), MMC (27.1\%), postinfectious (11.1\%), congenital $(9.7 \%)$, posterior fossa cysts $(9 \%)$, aqueductal stenosis $(7.6 \%)$ and others $(4.2 \%)$. 
Overall 59 patients (41\%) died. The frequencies of patients surviving 1,2, 10 and 20 years after first operation were 80 , 71,66 and $62 \%$, respectively.

The cause of death was evaluated in each patient. In 24 patients the cause of death was related to shunt treatment: Shunt infection was diagnosed in 18 and acute shunt dysfunction in 6 patients. 11 of 18 deaths associated with shunt infection occurred during the first postoperative year. In 27 patients the cause of death was unrelated to the shunt. However, in 8 patients the cause could not be determined.

89 patients were surviving more than 20 years after initial shunt operation. Of those long-term survivors, 4 patients (4.5\%) died 22-24 years after first operation. In 2 patients the cause of death was acute shunt dysfunction and in the other 2 patients the cause could not be determined.

Conclusion: Mortality in hydrocephalic patients is high, especially in the first postoperative years. But even after 20 years the mortality rate is significant high. Therefore, routine follow-up of long-term survivors remains necessary.

\section{FP092}

30 day return to OR after VP shunt operation: a quality measure?

Michael Handler, Sarah Graber, Claire Palmer, Todd Hankinson, Brent O'Neill, Corbett C. Wilkinson, Daniel Hyman (Aurora CO, USA)

Introduction: Quality measures should provide an opportunity for implementing improvement at an institutional level. Rate of reoperation within 30 days of pediatric shunt surgery is a commonly considered quality measure. We reviewed our experience to determine whether 30 day return to OR following pediatric shunt surgery met criteria as a quality measure, based on identification of opportunities for practice improvement.

Methods: Patients who underwent shunt surgery between 1998 and 2015 were identified using billing and scheduling codes. We collected retrospective data on patient demographics and shunt history, surgical details, shunt specifics, and 30 day return to OR. Patients who were lost to follow-up or with planned return to OR were excluded from the final analysis.

Results: We included 1999 shunt operations among 774 patients. $19.1 \%$ of operations were followed by a return to OR within 30 days $(\mathrm{n}=382)$. The most common reasons were shunt malfunction $(64.7 \%)$, catheter misplacement $(9.7 \%)$, infection $(8.1 \%)$, and isolated or loculated ventricles $(7.9 \%)$. Factors found to have a statistically significant association with 30 day return included age at surgery $(\mathrm{p}=0.008)$, number of prior shunt surgeries $(p<0.001)$, history of shunt infection $(p<0.001)$, timing of surgery (Jan-Jun vs.
Jul-Dec) ( $p=0.014)$, type of surgery (initial shunt placement vs. revision) $(\mathrm{p}<0.001)$, type of shunt revision (proximal vs. distal) $(\mathrm{p}<0.001)$, presence of multiple vs. single proximal catheters $(\mathrm{p}<0.001)$, proximal catheter entry location (right vs. left) $(p=0.044)$, and use of a new tract vs. re-use of an old tract at revision $(\mathrm{p}=0.017)$.

Conclusion: Most risk factors for 30 day return to OR were non-actionable and cannot be addressed through quality improvement initiatives. Others were determined by operative decision-making or pathology. The existing 30 day return to OR metric is inadequate on a stand-alone basis, and at best requires risk adjustment to be a useful quality measure.

\section{FP093}

Perinatal diagnosis of Dandy Walker malformation: treatment and outcome

James Ulrich, Samuel Ndoro, John Caird, Darach Crimmins (Dublin, Ireland)

Introduction: Dandy Walker malformation (DWM) consists of a cystic dilatation in the posterior fossa with associated cerebellar vermian hypopasia and often secondary hydrocephalus. It is usually diagnosed antenatally or in the perinatal period by diagnostic ultrasound and more recently MRI. We present a series of children with a diagnosis of Dandy Walker malformation who presented to us antenatally or at birth.

Methods: All liveborn children who attended the Pediatric Neurosurgery Department in Dublin with a diagnosis of Dandy Walker malformation were included. Their clinical notes and imaging were scrutinized.

Results: There were 26 children with a diagnosis at birth of Dandy Walker malformation. Median follow up is for 7.2 years (range 18 months to 18 years). There were 12 females.

On further more detailed imaging (post natal MRI) nineteen children were confirmed to have Dandy Walker malformations. Fourteen children with DWM had associated hydrocephalus, 2 had dysgenesis of the corpus callosum, absent septum and polymicrogyria, 1 had an associated encephalocele and one had grey matter heterotopia. 6 had non-CNS congenital anomalies including renal, thyroid and spinal problems. Sixteen children with DWM required surgery. Three had initial treatment of a cystoperitoneal (CP) shunt (one required fenestration of the cyst also). One had ETV with subsequent shunt. Twelve had a VP shunt (one of whom required an additional $\mathrm{CP}$ shunt.

Twelve $(63 \%)$ of 19 children with DWM were intellectually normal ( 2 of whom have mild balance issues). All of these children had CSF diversion surgery. Four children had severe developmental delay (1 had hydrocephalus). 
Three children had mild motor and learning difficulties. Appearance of the vermis, cyst size or presence of hydrocephalus on MRI did not affect developmental outcome.

The initial diagnosis was changed in 7 (27\%) of the 26 children (2 arachnoid cyst, 3 Blakes pouch cyst, 1 mega cisterna magna and one mega cisterna magna). Five of these had normal development.

Conclusions: Antenatal/perinatal diagnosis of DWM in our series was incorrect in 1:4 cases. This improves with antenatal and early postnatal MRI. Two thirds of children with DWM have normal or near normal development. One sixth has severe developmental delay. This is independent of hydrocephalus, cyst size or appearance of the vermis.

\section{FP094}

Survival of gravitational versus traditional nongravitational valves after primary ventriculoperitoneal shunt insertion in children: a retrospective cohort study Thomas Grainger, Jody O'Connor, Gregory James (London, United Kingdom)

Introduction: Gravitational valves -designed to reduce overdrainage by preventing siphoning- are increasingly being used for primary shunts in children. This long-term theoretical advantage is tempered by the suspicion that more complex valve designs are more prone to early failure.

Methods: Retrospective cohort study, examining all primary shunts -either gravitational valve (GV; Miethke PaediGAV) or non-gravitational valve (NGV; Codman Precision) inserted at our institution during the study period 2007-2014. Early failure was defined as need for shunt revision within 30 days of primary surgery. Late failure was defined as need for shunt revision at any point following 30 days. Chi square statistical analysis was used to compare groups.

Results: 207 primary shunts were inserted during the study period -36 GV and $171 \mathrm{NGV}$. Mean age of the GV group was 262 days (range 2-3561) against 783 days (range 1-5663) in NGV group ( $\mathrm{P}=0.013)$. The diagnosis profile differed between the 2 groups, with post-hemorrhagic ( $25 \%$ vs. $18 \%)$ and dysraphism-associated ( $28 \%$ vs. $12 \%$ ) hydrocephalus proportionally more common in the GV group and tumor-associated hydrocephalus $(24 \%$ vs. $3 \%)$ more common in NGV group. There were 5/36 (14\%) early failures in the GV group and 10/ $171(6 \%)$ in the NGV group $(\mathrm{P}=0.091)$. For late failures, there were $4 / 31(14 \%)$ events in the GV group with $45 / 161$ (28\%) in the NGV group ( $\mathrm{P}=0.078$ ). 1 year shunt survival in the GV group was $77.8 \%$ vs. $79.5 \%$ in the NGV group.

Conclusions: The overall 1 year survival was almost identical between the 2 groups, although there was a trend for more early failures in the GV group which was balanced by less late failures. In line with previous studies, there is little evidence currently to suggest superiority of one valve choice over another for primary shunts in children.

\section{FP095}

The individuality of intracranial arachnoid cysts in children - A single institute analysis

Elke Januschek, Andreas Röhrig, Sandra Kunze, Martina Messing-Jünger (Offenbach, Germany; Sankt Augustin, Germany)

Objective: Intracranial arachnoid cysts are benign spaceoccupying lesions, which may be symptomatic with increased size. In a large MR imaging series of 11,738 children a prevalence of $2.6 \%$ was found with a predominance in males and left temporal. The aim of our study was to analyze all surgically treated children with intracranial arachnoid cyst regarding size, location, symptoms, revision rates and underlying causes.

Methods: We retrospectively evaluated all consecutive patients with intracranial arachnoid cyst treated by surgery between $1 / 2009$ and 12/2015 in our department. Children, who were previously operated externally were excluded.

Results: 43 children (32 males, 11 females) were included. In $37.2 \%(n=16)$ the arachnoid cysts were located in the temporal fossa, with no side predilection. In 8 children the posterior fossa or suprasellar region were affected, in 5 the interhemispheric fissure and in 2, intraventricular and quadrigeminal cysts. We found one arachnoid cyst frontal over the convexity and one huge bihemispherical. In 27 cases, preoperative increase in size was observed. Most children became symptomatic by macrocephaly $(58 \%)$ and hydrocephalus, sometimes in combination. Five times clinical signs of an increased intracranial pressure were seen, 2 children showed a sunset phenomenon. As surgical modality we performed initially a microscopic (19) or endoscopic (17) fenestration, in 3 cases a shunt insertion or endoscopic fenestration combined with shunt insertion and one time an endoscopic fenestration and stent implantation were carried out. In the endoscopic group 5 secondary shunt implantations became necessary due to insufficient resorption of cerebrospinal fluid, in the microscopic fenestration group 2 times. In each group one re-fenestration was required. One uncomplicated cerebrospinal fluid fistula was seen. The follow up period ranges from 3 to 83 month.

Conclusion: As recommended in the literature, only symptomatic arachnoid cysts have been treated surgically. The preferred surgical procedure was endoscopic fenestration for suprasellar and intraventricular lesions, microscopic fenestration in temporal or posterior fossa cysts. The revision rate is higher in the endoscopic group, however, a re-fenestration is possible without additional risks. In case of insufficient resorption of cerebrospinal fluid implantation of a ventriculo- or cystoperitoneal shunt is the method of choice. This strategy is safe and provides an overall excellent outcome. 


\section{FP096}

Evaluation of the safety and efficacy of pediatric intraventricular endoscopy

Amy Louise Bowes, Josh King-Robson, William Dawes, Gregory James, Kristian Aquilina (London, United Kingdom)

Introduction: Intraventricular endoscopy has become an increasingly popular minimally invasive technique that may be applied to a variety of neurosurgical pathologies. The aim of the current study is to determine whether age at intervention influences the safety and efficacy of paediatric intraventricular endoscopy, as well as the need for definitive cerebrospinal fluid (CSF) diversion post-operatively.

Methods: In this retrospective cohort study, 287 paediatric patients aged up to 17 years of age underwent intraventricular endoscopy at Great Ormond Street Hospital (GOSH) from December 2005 to December 2014 (52 infants $=0-6$ months, 38 infants $=6-12$ months, 74 patients $=1-5$ years, 55 patients $=5-10$ years, 68 patients $=10$ years + ). Primary diagnosis, procedure type and complications were all recorded.

Results: 227 patients underwent neuroendoscopic intervention for the management of CSF-related disorders (79.1\%), including endoscopic third ventriculostomy (53.7\%; ETV) and for the treatment of intracranial cysts $(22.3 \%)$. Primary indication was not significantly different between age groups $(\mathrm{p}>0.05)$.

Following neuroendoscopy for CSF-diversion ( $\mathrm{n}=227$ ), a significantly higher rate of shunt insertion was observed in patients aged 0-6 months (63.0\%) when compared to older paediatric patients $(46.4 \%$, 6-12 months; $26.3 \%, 1-5$ years; 38.6\%, 5-10 years; $30.8 \%, 10$ years +$)(\mathrm{p}<0.001)$. Similarly, for patients who underwent ETV $(\mathrm{n}=171)$, post-operative shunt insertion was also significantly higher within the $0-6$ month cohort $(67.9 \%, 6$ 12 months; 47.6\%, 6-12 months; 19.6\%, 1-5 years; $27.3 \%$, 510 years; $23.3 \%, 10$ years +$)(\mathrm{p}<0.001)$.

No significant difference in complication rate $(12.2 \%)$ or infection rate $(2.4 \%)$ was observed between age groups ( $\mathrm{p}>0.05$, respectively). Of note, there was no perioperative mortality.

Conclusion: Intraventricular endoscopy is a safe neurosurgical intervention in paediatric patients of all ages, although it may be associated with increased shunt rates within young infants (0-6 months).

\section{FP097}

Is there any correlation between static and pulsatile intracranial pressure parameters and intracranial volumes in children and adolescents with Chiari malformation type I? Radek Fric, Kristian Eide Per (Oslo, Norway)

Introduction: In Chiari malformation type 1 (CMI), developmentally small posterior cranial fossa is believed to be one the key factors. However, intracranial volumetry has never been correlated to parameters of intracranial pressure (ICP), particularly pulsatile ICP.

Methods: Among CMI patients who underwent preoperative diagnostic ICP monitoring from 2006 to 2014, we selected those under 18 years of age at the time of presentation. We measured total intracranial volume (ICV), posterior cranial fossa volume (PCFV) and ventricular CSF volume (VV) from initial MRI using volumetry software. Radiological data were statistically correlated to parameters of static (mean ICP) and pulsatile (mean wave amplitude, MWA) ICP as analyzed by the software using automatic algorhitm for identification of cardiac beat induced intracranial pressure waves.

Results: Eighteen children and adolescents were identified, male:female ratio 11:7, median age 12 years (range 6-18) and median body mass index (BMI) 18.2 (range 15.3-32.7). Syringomyelia was present in $44 \%$ of patients, median tonsillar ectopy was $15 \mathrm{~mm}$ (range 7-20) under the level of foramen magnum. Median ICV was $1474 \mathrm{ml}$ (range 1374-1776), PCFV $185 \mathrm{ml}$ (range 152-236) and VV $17 \mathrm{ml}$ (9-86). Median ratio $\mathrm{PCFV} / \mathrm{ICV}$ was $12.3 \%$ (range $11-13.7$ ) and $\mathrm{VV} / \mathrm{ICV} 1.2 \%$ (range 0.6-4.8). Median value of mean ICP was $10.2 \mathrm{mmHg}$ (range 4.7-17.5) and of MWA $5.1 \mathrm{mmHg}$ (3.1-6.6). On statistical analysis using Pearson correlation, no significant correlation was found between intracranial pressure parameters and volume measurements. There was no significant correlation between ICP and BMI or degree of tonsillar ectopy, either.

Conclusions: Although a relatively small cohort of patients, the present analysis could not confirm any significant correlation between parameters of static / pulsatile ICP and relevant intracranial volumes in pediatric Chiari malformation type 1 .

\section{FP098}

Ventriculo-subgaleal shunt using an antibioticimpregnated catheter: a retrospective analysis of our institutional experience

Paolo Frassanito, Gianpiero Tamburrini, Luca Massimi, Simone Peraio, Rina Di Bonaventura, Massimo Caldarelli (Rome, Italy)

Introduction: Ventriculo-subgaleal shunt (VSS) is a temporary measure to manage hydrocephalus, eventually overcoming the risks of external ventricular drainage and subcutaneous reservoir. Methods: We performed a retrospective analysis of VSS performed at our Institution in the period 2013-2015. Antibioticimpregnated catheter (Bactiseal $^{\mathbb{R}}$, Codman) along with a right angle connector were used to build a patient-tailored shunt. In case of collapsed or tense subgaleal pouch with signs of elevated intracranial pressure, revision of the VSS was performed while tapping of the pouch was always avoided to reduce the 
risk of infection. Patients were monitored with serial ultrasounds.

Results: We collected 36 patients receiving VSS. Main indication was post-hemorragic hydrocephalus in 32 preterm babies, followed by post-infectious in 3 and systemic conditions contraindicating the implant of permanent shunt in a single case. One patient also received subdurosubgaleal shunt and another patient received an IV ventricle-subgaleal shunt. VSS controlled hydrocephalus in all patients for a period ranging from 15 days to 9 months (median 32 days). Revision was required in a half of the case. Complications were: one case of hemorrhage secondary to abrupt ventricular drainage, that did not require additional surgery, and two cases of infection in patients who underwent multiple surgical procedures (including endoscopy for multiloculated hydrocephalus). All patients were converted to a permanent shunt except for 2 patients who died because of complications of prematurity and one patient who was made shunt-free with endoscopic third-ventriculostomy.

Conclusions: VSS is a safe and effective method to control hydrocephalus in critical babies. Our policy, based on the use of antibiotic-impregnated catheter and avoidance of tapping the subgaleal pouch, contained the rate of infection to about $5 \%$ in this particular subset of patients. Management of patients harboring VSS resulted easier than other options, such as external ventricular drainage, to nurses and intensive care physicians.

\section{FP099}

\section{Endoscopic management of suprasellar arachnoid cyst} with associated ventriculomegaly

Jose M. Francisco Salomão, Antonio R. Bellas, Tatiana Protzenko Cervante, Flávia Abreu (Rio de Janeiro, Brazil)

Introduction: Suprasellar arachnoid cysts (SSAC) account for $1 \%$ of all the space occupying lesions and $15 \%$ of all arachnoid cysts in children. In $90 \%$ of the cases SSAC present with hydrocephalus and endoscopic fenestration is the gold standard in the treatment.

Material and Methods: Retrospective study of 21 children treated with endoscopic fenestration from 1997 to 2014. Only patients with SSAC associated to ventriculomegaly and with a minimum follow-up of 2 years were included. There was a small prevalence of males (12/9). Intracranial hypertension was present in 13 patients, macrocrania in 11, development retardation in 8 , endocrine compromise in 4 . In one a VP shunt has been previously inserted. The first 5 patients underwent a ventriculo-cystostomy (VC), while in remaining a ventriculocysto-cisternostomy (VCC) was performed.

Results: Failure of treatment was detected in 5 patients and 2 presented with early CSF fistula. In 3, VC was the initial treatment. In this group one or more reoperations were needed. One patient with bobbing-head doll syndrome required 3 reoperations. Three patients ultimately required CSF shunting and in two of this group, involvement of other cisterns was present. No remarkable change in endocrine disturbances was noticed. Postoperative complications were seen in 4 patients. There were no deaths.

Conclusion: Endoscopic fenestration is the treatment of choice for SSAC and VCC is preferable to VC. The success rate exceeds $70 \%$ and morbidity is low. Endocrine disturbances are irreversible and if isolated, surgery should not be indicated.

\section{FP100}

Cerebrospinal fluid drainage options for posthemorrhagic hydrocephalus in premature neonates

José Roberto Tude Melo, Rosane Klein Passos, Marcelo Liberato Coelho Mendes de Carvalho (Salvador, Brazil)

Introduction: Posthemorrhagic hydrocephalus $(\mathrm{PHH})$ is a major cause of death among low birth-weight premature neonates. The literature describes various cerebrospinal fluid (CSF) drainage techniques to alleviate $\mathrm{PHH}$; however, consensus has not been reached on the best approach. Here we describe a case series of premature neonates treated for $\mathrm{PHH}$ by CSF drainage to assess outcomes of different approaches.

Methods: A consecutive review of the medical records of neonates with $\mathrm{PHH}$ that were hospitalized between December 2009 and December 2014 was conducted, and all neonates treated with CSF drainage were included in this study. This single-center study was approved by the Brazilian Research Ethics Committee.

Results: Forty premature neonates treated for $\mathrm{PHH}$ by CSF drainage were identified. The median age and weight were 28 weeks and 1,105 g, respectively. The serial lumbar puncture (LP), ventriculosubgaleal (VSG) shunt, and ventriculoperitoneal (VP) shunt were the treatment of choice in $25 \%$ (10), 37.5\% (15) and 37.5\% (15) of the cases, respectively. Neonates treated with temporary CSF drainage by the LP or VSG shunt had lower median gestational age and lower birth-weight, as well as more severe clinical presentations than those treated with the permanent VP shunt $(100 \%$ vs. $66 \% ; p=0.004)$. The patients that received the VP shunt as the initial treatment $(n=15)$ had a higher incidence of complications than those treated for $\mathrm{PHH}$ with the VP shunt during the follow-up period (66\% vs. $16 \% ; p=0.01)$.

Conclusion: In the absence of established protocols for $\mathrm{PHH}$ treatment in premature neonates, CSF diversion should be tailored to each case with preference given to temporary CSF drainage in neonates with lower age and lower birthweight, while the permanent VP shunt should be considered in healthier, higher birth-weight neonates born closer to term. 


\section{FP101}

Combined endoscopic third ventriculostomy and choroid plexus coagulation in pediatric hydrocephalus

Radovan Mijalcic, Waleed Azab (Kuwait, Kuwait)

Introduction: Combined endoscopic third ventriculostomy and choroid plexus coagulation (ETV-CPC) procedure is currently evolving as an effective treatment modality in various types of neonatal and infantile hydrocephalus. Coagulation of the choroid plexus has been reported to increase the efficacy and success rates of ETV in this patient subpopulation. In this work we present a retrospective analysis of a cohort of patients in whom an ETV-CPC procedure was performed.

Materials and Methods: From a database of endoscopic procedures maintained by the authors, all patients undergoing ETV-CPC were retrieved. Demographic, clinical and neuroradiological data were collected and analyzed.

Results: Thirty one patients including 19 males and 12 females with an age range between 2 to 39 months were studied. In the 31 patients, the etiology of hydrocephalus was post-infectious in 8 , myelomeningocele with Chiari II malformation in 12, post-hemorrhagic in 6 , and VP shunt malfunction in 5 patients. Over a follow-up periods ranging between 2 to 17 months, the achieved total success rate of ETV-CPC was $54.8 \%$. In the various etiological subcategories of hydrocephalus, success rates were $62.5 \%$ (5 of 8 patients) in post-infectious, $58 \%$ (7 of 12 patients) in myelomeningocele with Chiari II malformation, 50\% (3 of 6 patients) in post-hemorrhagic, and $40 \%(2 / 5)$ in VP shunt malfunction. No correlation between age and success rate could be found.

Conclusions: ETV-CPC procedure is an effective tool within the armamentarium of the available surgical options for treating pediatric hydrocephalus. The procedure is usually used to treat a rather challenging subcategory of hydrocephalic patients in whom VP shunts and ETV alone are known to have lower success rates. Although the retrospective nature and small number of subjects in our study make firm conclusions difficult to be drawn, the success rates of ETV-CPC in this study are similar to some of those reported in the literature.

\section{FP102}

Fetal ventriculomegaly: what does it mean?

Patricia Barrio Fernández, Javier Pérez Suárez, Bienvenido Puerto Navarro, David Santamarta Gómez (Leon, Spain; Barcelona, Spain)

Introduction: Assessment of the width of the atria of the lateral cerebral ventricles is recommended as part of the routine anomaly scan. Ventriculomegaly (VM) is the most common anomaly of the Central Nervous System detected in fetal life. It is defined as a lateral ventricular diameter measuring greater than $10 \mathrm{~mm}$ at the level of the atria on an axial plane at any point during gestation, as measured by sonography or magnetic resonance imaging (10-15 $\mathrm{mm}$, mild or moderate $\mathrm{VM} ;>15 \mathrm{~mm}$, severe $\mathrm{VM})$. The prevalence of VM varies between 0.3 and 1.5 per 1,000 births, depending on the technique used for measuring, the evaluation of one or both lateral ventricles and gestational age at examination.

Methods: The relevant literature was reviewed giving particular interest in outcomes.

Results: Up to $50 \%$ of cases are associated with other abnormalities (structural defects 33-61\%, chromosomal aberrations $3-9 \%$ and congenital infection $5 \%$ ). The size of the ventricles and its progression during the pregnancy are the most important factors in the outcome (presence of another anomalies, neurodevelopmental delay, perinatal or neonatal death and need of postnatal ventriculoperitoneal shunt). The risk of progression of ventricular dilatation is about $16 \%$. Additional neonatal imaging is needed to be performed because prenatal imaging showed a false negative rate of $7.4 \%$. Confirmed mild isolated ventriculomegaly is related to a prevalence of neurodevelopmental delay of $7.9 \%$.

Conclusions: In spite of advancements in prenatal imaging and molecular diagnosis of fetus, the management of fetal ventriculomegaly is still limited. The detection of associated abnormalities is essential for proper prognosis assessment. Isolated mild VM in the prenatal period constitutes an easy-to-detect diagnosis but a difficult-to-assess condition. However, parents and pediatricians should be advised that it delays in different areas of neurological development may appear during the infant period.

\section{FP103}

Treatment of posthemorrhagic hydrocephalus in preterm neonates using ventricular drain reservoirs: safety and effectiveness

Martin Geerlings, Kian Liem, Erik van Lindert (Nijmegen, the Netherlands)

Introduction: Intraventricular hemorrhage in the premature neonate may evolve into posthemorrhagic hydrocephalus (PHH). The objective of this study was to evaluate the safety and efficacy of ventricular reservoirs for temporary PHH treatment and establish the rate of shunt dependency. Moreover, the effect of a new protocol for early PHH treatment, already at small ventricular dilatation, was analyzed.

Methods: Retrospective cohort study of premature neonates with PHH, born between January 2004 and 
December 2013, in whom ventricular reservoirs were inserted for intermittent CSF drainage. Treatment consisted of CSF diversion via lumbar punctures, ventricular reservoirs and finally by ventriculoperitoneal (VP-) shunts, and was initiated either with ventricular dilatation exceeding the $97^{\text {th }}$ percentile according to Levene (group 1 , low threshold) or $97^{\text {th }}$ percentile +4 millimeters (group 2, high threshold).

Results: A total of 273 infants with IVH were born of which 38 (14\%) had PHH, whereas ventricular reservoirs were inserted in 24 neonates. Another 14 neonates were treated exclusively with lumbar punctures and eventually a VP-shunt. Three reservoirs (13\%) became infected and were treated conservatively. In 6 patients $(25 \%)$, reservoirs were revised due to obstruction. Three infants died unrelated to reservoir complications. Finally, 11 infants (52\%) were still in need of CSF diversion by VP-shunts, median 7.5 (3-24) weeks after reservoir insertion; 4/9 neonates of group 1 and $7 / 12$ of group 2. No significant differences in complication rates have been identified. However, intracerebral hemorrhage due to a reservoir insertion, as diagnosed by ultrasound, occurred exclusively in two neonates of group 2 (high threshold).

Conclusion: Ventricular reservoirs can be used safely for temporary PHH treatment to escape definitive shunt insertion. Early (low threshold) treatment of PHH may lead to a reduction of shunt dependency, without a higher risk for reservoir or shunt complication.

\section{FP104}

\section{Radiological and neurological response rates in} aqueductal stenosis hydrocephalus treatment

Matthias Preuß, Stefan Schob, Ulf Nestler, Andreas

Merkenschlager (Leipzig, Germany)

Treatment of aqueductal stenosis (AQS) has undergone several paradigm shifts during the past decades. Currently endoscopic ventriculostomy (ETV) is recommended as treatment of choice. Several authors have addressed the issue of variable ETV success rates depending on age and pathogenetic factors. However, success rates have usually been defined as "ETV non-failure". Aim of the study was a retrospective analysis of radiological and neurological treatment response after ETV or VP-Shunting (VPS) in age-dependent subtypes of AQS.

Patients and Methods: 79 consecutive patients (median age 12.5 years, range 0-79 years) have been treated for MRIproven aqueductal stenosis. Neurological treatment success was defined by neurological improvement and, in childhood, head circumference. Radiological response was measured as Evan's index in follow-up MRI. Initial signs and symptoms, type of surgery and complications were analyzed.
Results: Four types of AQS have been defined with distinct age ranges and symptomatology: congenital Type-I $(n=23)$, juvenile chronic (tectal tumor-like) Type-II $(\mathrm{n}=23)$, adult acute Type-III $(\mathrm{n}=10)$ and adult chronic (normal pressure hydrocephalus-like) Type-IV $(n=23)$. Type I ( $<1$ years of age) is more successfully treated with VPS than with ETV $(81 \%$ vs. $50 \%)$. Treatment of chronic-juvenile Type II by ETV compared to VPS resulted in similar neurological response rate of $87.5 \%$ but showed radiological improvement in only 19\% compared to $57 \%$ after VP-Shunt. There has been no influence of persistent ventriculomegaly in Type II after ETV in contrast to VPS therapy for neurological outcome. Adult acute Type III responded excellent to ETV. Type IV patients responded neurologically in $70 \%$ after ETV, but radiological response was low $(5 \%)$.

Conclusion: AQS can be divided into four distinct age groups and types in regards of clinical course and symptomatology. Depending on the AQS type, ETV cannot be unequivocally recommended. Congenital Type-I AQS seems to have better neurological outcome with VP-Shunt whereas acute adult Type-III offers excellent ETV results. Juvenile chronic Type II still requires prospective investigation of long-term ETV outcome, especially when ventriculomegaly persists. Adult chronic Type-IV seems to result in similar outcome after VP-Shunt and ETV.

\section{FP105}

Endoscopic treatment of quadrigeminal cistern arachnoid cysts

Benicio Oton-De Lima, Marcio Ferreira Marcelino (Brasilia, Brazil)

Objective: Evaluate the effectiveness of endoscopy in the management of quadrigeminal cistern cysts.

Methods and Results: 23 patients with arachnoid cyst of the quadrigeminal cistern were surgically treated in the last 15 years. 16 were treated with endoscopic third ventriculostomy (ETV) and ventriculocystostomy (VC). In 4 the endoscopy was repeated and was found occlusion of the third ventriculostomy and patency of stoma between the cyst and the ventricle, and a ventricle peritoneal shunt (VPS) was done. In 7 patients the initial procedure was VPS and there was an increase of the cyst volume in 5 , which have been submitted to VC. In two there was persistent cyst enlargement and a cyst peritoneal shunt (CPS) was performed. There was clinical improvement in all patients, associated with the control of hydrocephalus.

Conclusions: The findings of our series show that the quadrigeminal cistern cyst can be effectively managed with endoscopy and the clinical improvement was associated with the control of hydrocephalus. 


\section{FP106}

Ventricle-sinus transversostomy in treatment of hydrocephalus

Kirill Sysoev, Vadim Ivanov, Alexander Kim, Constantine Samochernyh, William Khachatrian (St. Petersburg, Russia)

Currently, cerebrospinal fluid bypass surgery is the standard method of treatment of hydrocephalus, ventriculoperitoneal and ventriculoatrial shunting being the most often. However, efflux of cerebrospinal fluid from lateral ventricle of cerebrum to sinuses of dura mater (transverse sinus) appears to be more physiological.

Materials and Methods: Ventricle-sinus transversostomy in Russian Neurosurgical Institute n.a. professor Polenov had been executed 101 times within the period between 1983 and 2015. The results of treatment were compared with 1780 cases of other types of cerebrospinal fluid bypass surgery (ventriculoperitoneostomy and ventriculoatriostomy). Cerebrospinal fluid pressure and venous blood pressure in sinuses of dura mater (sagittal sinus, transverse sinus) had been monitored and position of ventricular catheter had been X-ray controlled in 49 patients in postoperative period. Shunt systems were adjusted according to the results of pressure infusion test.

Results: Ventricle-sinus transversostomy had been executed in 3\% initial cases of hydrocephalus, in $7.9 \%$ cases as cerebrospinal fluid bypass re-surgery and in $36 \%$ cases when cerebrospinal fluid bypass re-surgery had been repeated 4 and more times. The monitoring showed correlation dependence between cerebrospinal fluid pressure and venous blood pressure in transverse sinuses. As compared to traditional variants of bypass surgery, ventricle-sinus transversostomy caused drainage abnormalities less frequently. 14\% patients became independent of drainage after 1-7 years of medical surveillance.

Conclusion: Sometimes ventricle-sinus transversostomy is a single option in treatment of hydrocephalus in cases when ventriculoperitoneal and ventriculoatrial shunting is contraindicated.

\section{FP107}

\section{Intraoperative ultrasound guidance for shunt placement} in hydrocephalus

Marcel Kullmann (Tuebingen, Germany)

Introduction: Ventricular shunting operations are one of the most effective options for treatment of hydrocephalus in all age groups. However, there are avoidable complications, including misplacement of the ventricular catheter. Intraoperative Ultrasound Guidance (IOUSG) has been increasingly used for planning and definition of catheter trajectory, which includes catheter direction and length and in case of open fontanelle, intra-op real-time control of catheter position.

The aim of our study is to evaluate the benefit of IOUSG for the placement of ventricular catheter (VC).

Methods: A retrospective analysis of children's' data from June 2007 to July 2014 with the diagnosis of hydrocephalus was performed. 64 patients were included with primary insertion of a ventricular shunt. The mean age was 4 years ( 0 to 17). $50 \%$ females $(n=32)$ and $50 \%$ males $(n=32)$.

The postoperative catheter position was scored according to postoperative ultrasound or MRI: a) Position of the catheter in the middle of the ventricle, b) close to the medial or lateral wall or touching it, c) in the third ventricle or d) going to the contralateral side. The number of reoperations and reason of shunt dysfunction were recorded as well.

Results: In 19 cases (30\%) the VCwas placed occipitally, in 45 cases $(70 \%)$ frontally. In 39 cases $(61 \%)$ the VC was positioned in the middle of the ventricle, in 16 cases $(25 \%)$ close or touching the medial wall, in 5 cases $(7 \%)$ close or touching the lateral wall, in 3 cases $(5 \%)$ VCwas placed in the third ventricle and in one case $(2 \%)$ on the contralateral side.

In 4 cases $(6 \%)$ there was a catheter occlusion within the first eight weeks after operation. In 3/4 the catheter was initially positioned close or touching the medial wall and in $1 / 4$ in the middle of the ventricle.

Conclusions: Compared to data of freehand catheter placement the literature, we have a higher rate of intended ventricle position (91\%), a low rate of catheter revisions due to early obstruction (6\%) and about the same success rate as frameless navigated procedures (89\%). Optimal catheter placement seems to prolong catheter survival.

The advantage of ultrasound- guided VC placement compared to navigated procedures for VC placement are: no extra preoperatic imaging, no time loss for referencing or positioning, real-time intra-op images, no extra cost. Therefore it should become standard of care in pediatric CSF shunt surgery.

\section{FP108}

Endoscopic third ventriculostomy using 2-micron laser: single centre experience in $\mathbf{7 3}$ pediatric patients

Cahit Kural, Lisanne Lalla, Artemisia Dimostheni, Marcel Kullmann, Florian Ebner, Martin U. Schuhmann (Ankara, Turkey; Tuebingen, Germany)

Objective: Endoscopic third ventriculostomy (ETV) is well established for the treatment of obstructive hydrocephalus. ETV is usually performed by mechanical creation of the connection between third ventricle and basal cisterns. We present a clinical series of pediatric patients using the 2-micron continuous wave laser to create the stoma (Laser-ETV) with regard to safety, efficacy, and follow-up. 
Methods: Laser-ETV was performed in 73 pediatric patients aged 3 days to 17 years. For preoperative decision making and trajectory planning a high resolution T2 weighted MRI was obtained. Ventricular puncture was always ultrasound-navigated. 87 procedures were performed since tumor biopsy $(n=7)$, endoscopic tumor resection $(n=1)$, cyst fenestrations $(\mathrm{n}=5)$, and septostomy $(\mathrm{n}=1)$ were performed alongside ETV with the same burhole.

Result: Mean procedure time (including cases with additional procedures) was $48 \mathrm{~min}$ (range 17-90 $\mathrm{min}$ ). The mean follow up was 36 months. No complications $(0 \%)$ related to the use of laser for stoma creation were observed. Early minor complications unrelated to laser ETV were observed in $6(8 \%)$ cases (either related to cyst fenestration/tumor resection or to burhole). 2 children required early VP shunt. During followup, 7 further VP shunts (compensated hydrocephalus despite open ETV) were done, 1 case got re-ETV followed by shunt because of recurrent stoma closure. 1 case needed CSF fistula repair. Thus the long-term success rate of Laser-ETV, defined as absence of pressure active or compensated hydrocephalus, was $86 \%$.

Conclusion: Laser ETV using 2-micron continuous-wave laser is fast, safe, and effective in children, with no laser related complications and low overall complication rate. Additional instrumentation like balloons for dilatation is not needed and laser fibers are reusable.

\section{FP109}

Treatment of hydrocephalus in fourth ventricle tumors: our experience in a series of 171 patients and review of the literature

Paola Peretta, Paola Ragazzi, Pierpaolo Gaglini, Christian Carlino, Mario Cacciacarne, Valentina Pennacchietti (Turin, Italy)

Introduction: At the time of diagnosis, hydrocephalus is present in approximately $70 \%-80 \%$ of patients with fourth ventricle tumors and there is no consensus regarding optimal management. There are multiple strategies for preoperative management of hydrocephalus in these children: medical therapies, direct microsurgical approach to the tumor, external ventricular drainage (EVD), endoscopic third ventriculostomy (ETV) and ventriculo-peritoneal shunt (VPs). We present our experience comparing to the literature.

Methods: We analyzed the treatment of hydrocephalus in 171 children operated for fourth ventricle tumors at Pediatric Neurosurgery of Regina Margherita Children Hospital during the last 20 years, 113 males and 58 females. The medium age was 81 months (1 month-220 months). The isthology was: 73 pilocytic astrocytomas, 67 medulloblastomas, 26 ependymomas and 5 various. Hydrocephalus was present in 128 patients $(74.8 \%)$. Seventy-five patients were treated before microsurgical approach to the lesion: 52 by ETV at mean time of 72 hours before (12-230 hours), 17 patients by EVD and 6 patients by VPs.

Results: At follow-up 139 patients are shunt free (81\%). Among patients treated by ETV we had one intraventricular bleeding and one bleeding of the cystic part of the tumor. We had no other complications. The percentage of patients with VPshunt in this group was $17.5 \%$. One patient with EVD had a CSF infection, treated by both intraventricular and intravenous antibiotics. VPshunt was inserted in 7 children treated by EVD.

Conclusion: In our experience ETV was a safe and efficacy technique to control hydrocephalus before surgery. The anatomy was always recognizable and feasible to the fenestration of the floor of the third ventricle. EVD may be convenient in case of metastatic lesion or conteporary to the surgery when patient is scheduled in short time.

\section{FP110}

Neonatal reservoir in the treatment of pediatric hydrocephalus - 10 years study

Dana Dúbravová, Eva Štefánková, Michal Petrík, Martin Smrek, Jan Trnka, Frantisek Horn (Bratislava, Slovak Republic)

Introduction: Neonatal reservoir is used predominantly for the treatment of hydrocephalus in preterm newborns. Any surgical intervention in this particular group of patients is associated with high complication rate, especially infection. In this study, authors assess the overall outcome and complication rate of the procedure.

Methods: The study consists of 66 consecutive cases of patients admitted to our hospital in years 2005-2015. In all patients, the neonatal ventricular resevoir was implanted, and the type of the reservoir $(35 \mathrm{~mm}, 40$ $\mathrm{mm}, 45 \mathrm{~mm}$ ) was chosen based on the patient's age and ventricular size.

Results: From all patients included in the study, $82 \%$ had posthemorrhagic hydrocephalus ( $\mathrm{n}=53), 12 \%$ congenital hydrocephalus $(\mathrm{n}=8)$, and $6 \%$ of other etiology (postinfective and meningomyelocele). The patients achieved $25^{\text {th }}-41^{\text {st }}$ week of gestation (mean 31, median 30). The mean age at the surgery was 1.99 month (median 1 month), and mean corrected age at the surgery was $37^{\text {th }}$ week of gestation. The overall complication rate was $9 \%$, of which infection rate was $6 \%$. No further permanent treatment was needed in $20 \%$ $(n=13)$, shunt conversion was performed in $55 \%(n=36)$, ETV in $10 \%$ and combined endoscopic and shunt treatment in $10 \%$. In $46 \%$ of patients, we performed more than 10 reservoir taps. Nor the etiology of hydrocephalus, neither the achieved gestational age did not significantly influence the complication rate $(\mathrm{p}=0.188$ and $\mathrm{p}=0.647$ respectively) or the need for further treatment $(\mathrm{p}=0.228$ and $\mathrm{p}=0.278$ respectively). 
Conclusion: Due to low complication rate and good overall outcome (no need for shunt conversion in $30 \%$ of patients) we consider neonatal reservoir as an optimal primary treatment of hydrocehalus in preterm newborns. If further treatment is necessary, endoscopic approach should be considered with conversion to shunt in older age if required, to prevent numerous shunt complications in newborns.

\section{FP111}

Neuroendoscopic foraminoplasty for the treatment of unilateral hydrocephalus

Michal Petrik, Memet M. Özek (Istanbul, Turkey)

Introduction: Unilateral hydrocephalus is the most common form of multiloculated hydrocephalus. In pediatric age group intraventricular hemorrhage and infection are the most common causes of this entity. The treatment is the fenestration of the isolated lateral ventricle with CSF pathways. Septostomy and foraminoplasty are the methods of treatment. We present our data of neuroendoscopic foraminoplasty of foramen Monro for the treatment of this group of patients.

Methods: We performed neuroendoscopic foraminoplasty of foramen Monro in 16 pediatric patients diagnosed with unilateral hydrocephalus. All patients were evaluated with TSE T2 and 3D volumetric T2 MR sequences. Patients with membraneous obstruction of foramen of Monro are selected for foraminoplasty procedure.

Results: Clinical results in all cases were excellent with decrease of ventricular size and corrected septal shift postoperatively. The asymmetry of the lateral ventricles remained existing. We report no complications in our series

Conclusions: Neuroendoscopic foraminoplasty of foramen Monro is safe method in selected cases and should be considered as a primary surgical approach in diagnosis of membranous obstruction at the level of intraventricular foramen in patients with unilateral hydrocephalus. Precise MRI preoperative evaluation and selection is recommended. Neuroendoscopic foraminoplasty of foramen Monro reestablishes pressure equilibrium by reopening natural anatomical conduit for cerebral fluid.

\section{FP112}

\section{Functioning of children with hydrocephalus and their} families

Lucyna Szefczyk-Polowczyk, Marek Mandera (Katowice, Poland)

Introduction: Although hydrocephalus is one of the most common diseases demanding neurosurgical treatment of childhood, only a few studies analysing the influence of hydrocephalus on the biopsychosocial aspects of life have been published in the literature till now.
Aim: To examine the influence of hydrocephalus on selected aspects influencing life of a child and the whole family.

References and Methods: 711 children were treated for hydrocephalus between 2000-2010 in the Department of Paediatric Neurosurgery in Katowice. Parents of 130 children were asked to fulfil the authorial survey. The questions were focused on the functioning and well being of the child and the whole family. By employing Barthel scale, the level of independency of a child was determined.

Results: Children with posthemorrhagic hydrocephalus have reached lower level of independency than children with congenital hydrocephalus. Also the number of surgical procedures (shunt's implantation or revisions) influenced negatively the level of independency. Nearly a quarter of children with hydrocephalus have grown up in a single-parent family or in the children's home. However no significant correlation between the level of child's independency and the integrity of their family was found. More than half of the mothers resigned from a professional activity in aid of taking care of the children. Seventy-five percent of the parents have not received any psychological assistance.

Conclusions: Diagnosis of hydrocephalus has a significant impact on the family's life in economic, psychological and social sphere. Occurrence of posthaemorrhagic hydrocephalus and number of surgical procedures influenced negatively the independency of children with hydrocephalus.

\section{Poster Presentations}

\section{Session 1: Spinal dysraphism \& Session 3: Spine}

\section{PP01}

Shortening vertebrotomy for recurrent tethered cord syndrome in children

Kirill Sysoev, Eugene Khodykin, Edward Ulrich, William Khachatryan (St. Petersburg, Russia)

Introduction: Tethered cord syndrome (TCS) caused by spinal cord tension due to tethering of its caudal part in the lumbosacral spinal canal. The main purpose of surgical treatment of TCS is to release the structures of spinal cord from excessive tension. The traditional method of TCS treatment involves microsurgical elimination of tethering factors and excision of the filum terminale. In the cases of recurrent TCS surgical manipulations raise the risk of damage of spinal cord and its roots and in some cases tethered cord release is impossible. The shortening vertebrotomy was proposed as an alternative to microsurgical treatment for the recurrent tethered cord syndrome in adults. There are no reports of using shortening vertebrotomy in children with TCS.

Subject: 6 years old boy with history of myelomeningocele repair was examined. Complaints were lumbar pain, leg 
weakness, urinary and fecal incontinence. MRI showed multilevel spine and spinal cord malformations: spina bifida, tethered spinal cord at L5-S1 level, butterfly L2 vertebra, type II split cord malformation at L2 level, scoliotic spinal deformity. Methods: Transpedicular L2 vertebra resection, transpedicular fixation at Th12-L4 levels and contraction with decrease in the height of the vertebral column for $20 \mathrm{~mm}$ were performed.

Results: Reduction pain, increase in muscle strength of the lower limbs and the better control over bowel and bladder functions were observed within 2 years of follow-up after surgery. Thus, regress of TCS was observed after spine shortening without manipulation on the tethering site.

Conclusions: The shortening vertebrotomy can be considered as an alternative method to surgical treatment for some children with recurrent TCS when additional indications exist.

\section{PP02}

Clinical outcomes in children with caudal, dorsal and transitional lumbosacral lipoma

Lindy May, Adam Kucznski, Dominic Thompson, Jo Wray (London, United Kingdom)

Introduction: Lumbosacral lipoma is a rare form of neural tube defect. Although a degree of clinical dysfunction is inevitable due to dysplasia and "tethering" of the terminal spinal cord by the lipoma, clinical outcomes are variable, and there is a paucity of literature regarding the outcomes associated with lipoma types, making prediction of individual long term morbidity difficult. This study aims to identify if a particular lipoma type is associated with higher morbidity and with specific clinical deficits.

Methodology: Data was obtained from 54 children between the age of 5-18 years of age with caudal/ dorsal/ transitional lumbosacral lipomas. The clinical data collected included the presence of a syrinx, abnormal neurology, abnormal sphincter function including the requirement for CIC (Clean Intermittent Catheterization) and orthopedic abnormalities. SPSS was used to compare lipoma type with individual outcomes and the presence of a syrinx.

Results: Urological signs were the most common and orthopedic the least for all lipoma types. Overall morbidity was lower for dorsal and higher for transitional lipomas. Caudal lipoma was a risk factor for urological and bowel signs.

Conclusion: Urological problems are particularly common in all lipoma types. This is consistent with the understanding that nerves supplying the bladder are the first to be affected clinically by a tethered cord. Cases of transitional lipomas may be more affected than caudal and particularly dorsal lipomas. This may reflect greater involvement of nerve roots and rotation of the spinal cord in transitional lipomas. Children with transitional lipomas may require more surveillance and support than children with dorsal and caudal lipomas.

\section{PP03}

Multiple hereditary osteochondromatosis with spinal cord compression: a case report

Oscar Garcia, Jose Mireles, Natalia Sanchez, Miguel Chagolla, Sergio Orozco, Pedro Silva (Leon, Mexico; Queretaro, Mexico)

Introduction: Osteocartilaginous exostosis or Osteochondroma, is the most common benign tumor of the bone. This entity is an inherited autosomal dominant condition called hereditary multiple exocytoses (HME) that is characterized by the presence of multiple osseous prominences. Spinal cord compression resulting from osteochondroma is an extremely serious complication. The neurological presentation is slow due to the growth characteristics of the tumour. The symptoms are myelopathy and paralysis.

Case Description: An 8 year old boy diagnosed with $\mathrm{MOH}$, starts with paraparesis in the left foot, until the complete paralisis in both legs.

In CT we distinguish bony findings that were compressing the spinal cord at the level of the 3rd thoracic vertebrae and in the MRI we found a hydrosyringomyelic collection that involves the conus medullaris.

One month after surgery we asked for a Control CT in which we can observe the complete remotion of the bony and fibrocartilaginuos tumor from the spinal canal and in the MRI we found the resolution of the medular compression, and changes of the intensity of the signal in $\mathrm{T} 2$ sequence suggesting cronic myelopatic changes, with a reduction of the hidrosyryngomielic cavity in the caudal portion.

Two months after surgery with one month of rehab he recovered the strength in both legs that allow him to walk.

Conclusions: Due the location of the tumor in the thoracic portion of the spinal cord we make an anterior approach so we can expose the vertebral body and identified the thoracic vertebral bodies to have a complete corpectomy of the normal bone and the fibrocartilaginuos tumor that is located inside of the vertebral conduct.But nevertheless the intraoperative monitorization and magnification are essentials, so we can have the complete remotion of the tumor without damaging the spinal cord or any nerve root or structure adjacent.

\section{PP04}

Structural-molecular changes in lymphocytes in children with congenital disorders of the central nervous system Pavlo Plavskyi, Tetyana Malysheva, Daria Bezruchko, Sergiy Andreev, Oksana Chernenko, Viktoria Vaslovych, Olga Ivanova, Anna Shmeleva (Kiev, Ukraine)

Introduction: The congenital malformations are combined with tumours of the central nervous system (CNS). The 
congenital malformations in $20 \%$ of cases are caused by molecular dysfunctions that are not sufficiently understood.

Objective: Improvement of morphological diagnosis by comparing cytogenetic and morphological data in complex CNS congenital malformations.

Materials and Methods: The study involved 58 children: 32 girls and 26 boys with congenital malformations operated on cerebral spinal hernias at the Romodanov Neurosurgery Institute (Ukraine) in 2012-2014. In 36 cases the CNS malformations were combined with pathology of internal organs and tumours (15\%). The 9 cases ended lethally. We had 40 cases of morphological and molecular (chromosomal aberrations of lymphocytes culture) and 10 of them were the comparison group.

Results: The cerebral spinal hernias (CSH) were found in $37.9 \%$ of cases, $62.1 \%$ of the cases were combined with Arnold-Chiari anomaly $-22.4 \%$, polymicrogyria $-22 \%$; Dandy-Walker syndrome $-0,6 \%$; fixed spinal cord $-34 \%$; hydrocephalus $-44.8 \%$, hypoplasia of the corpus callosum $8.6 \%$, malformations of cerebral vessels (vascular tissue dysplasia) $-8.6 \%$; syringomyelia $-1.4 \%$. The malformations of the heart $-6.9 \%$, the defects of the skeletal system $-10.3 \%$. The defects of histoarchitectonics, ectopic tissue and cell proliferation, the formation of cavities, vascular malformations were found morphologically. The percentage of aberrant metaphase lymphocytes in the study group $-10.95 \%$; in the comparison group $-0.8 \%$. The increasing of frequency of aberrant metaphases was found in children with dysraphia: the karyotype of most metaphases - 44XX (44XY), and in $12 \%-43 \mathrm{XX}(43 \mathrm{XY})$, in the comparison group - 44XX $(44 X Y)$ in $100 \%$ of the analyzed metaphases. The identified dicentric chromosomes were metabolic chromosomal aberrations and microsatellite forms.

Conclusions: Neural tube defects are not associated with sex. In cases of dysraphia there was often blood group A (II) and usually negative $\mathrm{Rh}$ factor. The dicentric chromosomes were found in cases with combined dysraphia and tumors $(t>1,96$, relative to the comparison group). After clinical and morphological comparison it was established that system molecular genome restructuring is typical for combined dysraphia and CNS tumours.

\section{PP05}

Anterior intradural tumors of spine in children: endoscopy guided resection with neurophysiological monitoring by posterior approach

Ricardo Gepp, Marco Sainz, Enio Comerlato (Brasilia, Brazil)

Anterior intradural tumor of the spine is a challenge to the neurosurgeon, especially in children. The need for tumor removal is limited by the position of the spinal cord and the risk of neurological deficit due to spinal cord retraction during surgery. The performance of anterior approach of the spine is more difficult in children and there are risks of spine instability. The posterior surgical approach may limit the complete removal of the tumor and pieces may remain in the primary site due to incomplete visualization. The use of the endoscope in spinal surgery is still poorly described. The association between spinal endoscopy and neurophysiological monitoring allows better tumor visualization and lower risk of retraction of the spinal cord, decreasing the neurological risk.

In this study the authors described the experience gained from the posterior approach for two extramedullary intradural lesions with the aid of endoscopy in children.

Case Reports: The review of the medical records and surgical data from two children with previous intradural tumors spinal cord was performed. The surgical technique used in both cases began with osteoplastic laminotomy of the affected levels with lateral extension, removing small part of the facet joint on the side of tumor expansion. After opening the dura mater paramedian, began resection of the tumor by microsurgery. The endoscope lens $30^{\circ}$ was used to perform the inspection and help to perform complete resection. The surgeries were performed under neurophysiological monitoring, using somatosensory evoked potential and motor potential with $\mathrm{M}$ and $\mathrm{D}$ waves. Both cases had good results with complete resection and without neurological deficits.

Discussion: The endoscope primarily favored the tumor visualization in these cases and facilitated the search for tumor remnants. Children were 12 to 14 years old ages at the time of surgery and it was possible to take out the tumors completely with less bone resection, avoiding post-operative spinal deformity. Both lesions were located anterior to spinal cord, one dermoid tumor and other was a meningioma. The bleeding was less than $300 \mathrm{ml}$ in both cases, this value could be greater if there was need of bone resection and spinal fusion. There were no neurological deficits after surgery. Control images showed the complete resection of the lesions.

Conclusion: Microsurgery assisted by endoscopy is a safe and effective method to control resection of intradural anterior tumors, and associated with neurophysiological monitoring becomes a secure method of approach to this type of tumor.

\section{PP06}

\section{Foramen magnum stenosis in patients with achondroplasia}

Krešimir Saša Đurić, Ivan Pašalić, Jakob Nemir, Marjan Rožanković, Sanda Huljev, Miran Cvitković, Hrvoje Jednačak (Zagreb, Croatia)

Introduction: The authors are presenting their experience in treating pediatric patients with achondroplasia and stenosis of foramen magnum emphasizing neurosurgical 
relevance. Surgeries are performed at the Department of Neurosurgery Medical faculty in Zagreb during the last five years.

Methods: The study is based on retrospective analysis of continuously updated database that includes patient history, radiological files and surgical reports. Factors included in investigations were age, gender, symptoms, neurosurgical focus and postoperative course. The outcome of interventions is presented.

Results: Starting in the 2000 till 20155 patients with achondroplasia were surgically treated at the Department of Neurosurgery Medical faculty in Zagreb. Six surgical procedures were performed, there were three male and two female patients with age range from 5 months to 13 years. Presenting symptoms were ventriculomegalia with signs of chronic hydrocephalus (4 pts.), one patient had suden apnea attacks, and one pt. was diagnosed with external hydrocephalus. Foramen magnum decompression alone was performed in 4 pts. and in two pts. foramen magnum decompression with resection of posterior arch of atlas. One patient underwent two surgeries, first at 5 months of age when subduroperitoneal shunting was performed due to external hydrocephalus followed by foramen magnum decompression 7 months later due to ventruculomegaly. The follow up ranged from 62 to 2 months. There were no complications and symptoms resolved in all cases as the clinical and neuroradiological follow up showed.

Conclusion: Achondroplasia is rare genetic disorder with frequency between 1:30000 newborns with main morbidity caused from bony compression of foramen magnum and respiratory failure. In our series we had no patients with neurological evidence of upper cervical myelopathy due to significant foramen magnum stenosis. All patients had clear CSF flow disturbances and one pt. had respiratory difficulty that couldn't be explained by some other cause other than cervicomedullary compression. Simptoms diminished after FM decompression without duraplasy, there were no complications.

\section{PP07}

Atypical myelomeningocele associated with prominent vascularity

Julia Pavaine, Andrea Rossi, Armando Cama (Manchester, United Kingdom; Genova, Italy)

Introduction: Incidence of Open Spinal Dysraphisms (OSD) has decreased due to improvement in prenatal diagnosis and folic acid supplement during pregnancy, however occurs. Our purpose is to demonstrate two very rare atypical cases of myelomeningocele (MMC) associated with prominent vascularity. There are no similar cases in literature published to date.
Methods: Two male neonates with OSD underwent spinal MRI exams on the first day of life, at the Royal Manchester Children's Hospital and at IRCCS Instituto Giannina Gaslini respectively. First neonate also had spinal Doppler US examination and contrast enhanced time resolved 3D MR angiography (TWIST). Both infants underwent surgical repair of MMC without complications.

Results: Radiological exams revealed large MMC with septations for one neonate and hemi-MMC for another. Prominent blood vessels were identified within the herniated portion of the cord for both neonates. TWIST sequence did not reveal spinal AV-fistula or AVM for the first neonate. During surgical procedure for the same neonate dilated tortious vessel was seen within the herniated portion of the cord/dysplastic neural elements. No AVM or AV-fistula was identified.

Conclusion: Myelomeningocele could be rarely associated with prominent vascularity requiring careful pre-operative radiological investigation and less invasive surgical approach.

\section{PP08}

Composite type of split cord malformation: report of 2 cases and literature review

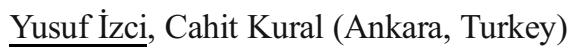

Introduction: Split cord malformation (SCM) is a rare form of spinal dysraphism. SCM may involve single or multi-level in the spinal cord. Different type of SCM also may be seen in the different levels. In this poster, we present 2 children with composite type SCM.

Case Report: Two children were presented hypertrichosis in the back. One of them had type 1 SCM at 2 different level and type $2 \mathrm{SCM}$ at the other level. Other child had type $1 \mathrm{SCM}$ at one level and type 2 at the other level of the spine. Both of them underwent for surgical treatment and all septums (bony and fibrous) were removed with microsurgical technique and the filum terminale was also cut. No complication had been occured.

Conclusion: Composite type SCM is very rare and can be detected if the whole spinal cord is scanned with magnetic resonance imaging. Surgical treatment should be performed in the same session in order to reduce the risk of tethered cord syndrome in adulthood.

\section{PP09}

Open spinal dysraphism associated with diastematomyelia: report of 2 pediatric patients

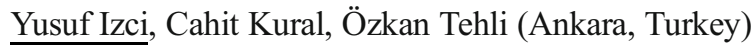

Split cord malformation is a rare type of congenital spinal malformations and may be associated with open spinal dysraphisms. In this poster, we present 2 newborns who were opered for open spinal dysraphism and diastematomyelia was detected in these patients. One child 
had myeloschisis and diastematomyelia and the other had myelomeningocele and diastematomyelia. Both of them underwent spinal CT scan for the evaluation of the malformation before surgery. Removal of bony septum and closure of the open spina bifida were performed during the surgical treatment. Intraoperative electrophysiological monitoring was used for the preservation of the neural structures.

Open spinal dysraphisms may be associated with split cord malformations and detailed radiological investigation (computed tomography or magnetic resonance imaging) should be performed before surgery in order to better surgical planning.

\section{PP10}

Kyphosis and angulation developed after surgery Cervical intramedullary mass resection

Ozkan Tehli, Cahit Kural, Mehmet Can Ezgu, Mehmet Ilker Ozer, Yusuf Izci (Ankara, Turkey)

Introduction: Tumors of the central nervous system are common in the pediatric population and constitute the second most prevalent tumor type of childhood. Spinal cord tumors are a relatively rare diagnosis, accounting for $1 \%$ to $10 \%$ of all pediatric central nervous system tumors.

Method: Our patient is a 9 years old male. The patient was presented with a motor function loss which has begun 2 weeks ago at left arm and leg. A servical MRI was performed and MRI showed an intramedullary mass lesion, localized between $\mathrm{C} 3$ and $\mathrm{C} 4$ cervical vertebrae. Somatosensory evoked potentials were normal. We operated the patient, and it contains C3-C4-C5 laminotomy and mass resection. The histopathological report defined a low grade glial tumor.

Result: We performed follow up MRIs on 3, 6, and 10 months after the surgery. MRIs and CT scan showed that a kyphotic deformity and angulation started to develop after surgery. We performed a second operation to correct the cervical posture, and made a posterior stabilization to the $\mathrm{C} 2-\mathrm{C} 3-\mathrm{C} 4-\mathrm{C} 5-\mathrm{C} 6-\mathrm{C} 7$ vertebrae. After the surgery, the cervical posture was corrected appearently.

Conclusion: In children, after laminotomy surgery, kyphosis and angulation may develop progressively in time. Therefore, a close follow up may be necessary to detect angulation even after a laminotomy surgery.

\section{PP11}

\section{An unusual cause of cyclical vomiting syndrome}

William White, Veejay Bagga, David Campbell, Anthony Hart, Hesham Zaki, Saurabh Sinha, John McMullan, Shungu Ushewokunze (Sheffield, United Kingdom)

Cyclical vomiting syndrome (CVS) is a functional disorder characterised by recurrent episodes of profuse vomiting separated by symptom-free periods ${ }^{[1,2]}$. The aetiology and pathogenesis of CVS is unknown however is thought to be linked with migraine as up to $87 \%$ of children with CVS have a migraine association ${ }^{[1]}$. The diagnosis of cyclical vomiting syndrome is one of exclusion with an investigation algorithm to exclude gastrointestinal and central causes of vomiting. Chiari malformation type-I is a craniocervical junction disorder in which there is cerebellar tonsil ectopia below the foramen magnum and into the spinal canal ${ }^{[3]}$. It is a relatively common condition, affecting an estimated $0.5-3.5 \%$ of the general population ${ }^{[3]}$.

In the literature there is only one report that alludes to a link between a type I Chiari malformation and $\mathrm{CVS}^{[1]}$. There are no reports on the outcome of surgery in these patients.

Here we report a case of a 12 year old boy with one year history of CVS. An MRI scan showed a type I Chiari malformation. He underwent a craniocervical decompression after all other organic causes of his vomiting were excluded. An immediate benefit was seen post-operatively in terms of vomiting frequency. At 6 months follow-up, he reported no further vomiting episodes and had returned to full-time education. This is the first reported case of successful surgical treatment of a patient presenting with CVS and a type I Chiari malformation.

\section{References:}

${ }^{1}$ Sunku B. Cyclic Vomiting Syndrome. Gastroenterology and Hepatology. 2009; 5(7): 507-515

${ }^{2}$ Tan MLN, Liwanag MJ, Quak SH. Cyclical vomiting syndrome: Recognition, assessment and management. World Journal of Clinical Paediatrics. 2014; 3(3): 54-58

${ }^{3}$ Arnautovic A, Spalvski B, Boop FA, Arnautovic KI. Paediatric and adult Chiari malformation Type 1 surgical series 1965-2013: a review of demographics, operative treatment, and outcomes. Journal of Neurosurgery: Paediatrics. 2015; 15: $161-177$

\section{PP12}

Cervical traumas in young population during sport activities - Radiological evaluation

Nikolaos Syrmos, Argyrios Mylonas, Georgios Gavridakis (Heraklion-Crete, Greece; Thessaloniki, Greece)

Background: Radiological evaluation is essential in spinal trauma patients during sport activities

Aim: Aim of this study was to analyze the radiological evaluation and the role of $\mathrm{x}$-ray, CT and MRI findings in 20 young patients $(<20$ years $)$ with cervical traumas during sport activities.

Material and Methods: During a 10 year period (20022012) we analyzed 20 patients with cervical traumas during sport activities (amateur and professional). 15 male, 5 
female, mean age 14, range from 8 to 20 years. All of them $20,100 \%$ had admission $\mathrm{x}$-rays and CT-scan that confirmed the presence of cervical traumas. 10 patients had also MRI-scan (group 1), 10 patients only x-rays and CTscan (group 2). We analyzed also the type of sport activity -amateur (group a, 15 patients), professional (group b, 5 patients)- then the timing of injury, recent trauma (group I, 27 patients) and chronic trauma, more than 7 days after the initial injury (group II, 3 patients).

Conclusions: Further studies were warranted but the combination of CT and MRI is very useful for the evaluation and for the therapeutic approach of spinal trauma patients.

\section{PP13}

Thoracic spine traumas in young population during sport activities - Radiological evaluation

Nikolaos Syrmos, Argyrios Mylonas, Georgios Gavridakis (Heraklion-Crete, Greece; Thessaloniki, Greece)

Background: Radiological evaluation is essential in spinal trauma patients during sport activities.

Aim: Aim of this study was to analyze the radiological evaluation and the role of $\mathrm{x}$-ray, CT and MRI findings in 6 young patients ( $<20$ years) with thoracic spine traumas during sport activities.

Material and Methods: During a 6 year period (20062012) we analyzed 6 patients with thoracic spine traumas during sport activities (amateur and professional). 5 male, 1 female, mean age 18, range from 16 to 20 years. All of them $6,100 \%$ had admission $\mathrm{x}$-rays and CT-scan that confirmed the presence of cervical traumas. 5 patients, had also MRI-scan (group 1), 1 patient only x-rays and CTscan (group 2). We analyzed also the type of sport activity -amateur (group a , 4 patients), professional (group b, 2 patients)-, then the timing of injury -recent trauma (group I, 5 patients) and chronic trauma more than 7 days after the initial injury (group II, 1 patient).

Conclusions: Further studies were warranted but the combination of CT and MRI is very useful for the evaluation and for the therapeutic approach of spinal trauma patients.

\section{PP14}

Lumbar spine traumas in young population during sport activities - Radiological evaluation

Nikolaos Syrmos, Argyrios Mylonas, Georgios Gavridakis (Heraklion-Crete, Greece; Thessaloniki, Greece)

Background: Radiological evaluation is essential in spinal trauma patients during sport activities.

Aim: Aim of this study was to analyze the radiological evaluation and the role of xray, CT and MRI findings in 12 young patients $(<20$ years) with lumbar spine traumas during sport activities.
Material and Methods: During a 10 year period (2002-2012) we analyzed 12 patients with lumbar spine traumas during sport activities (amateur and professional). 8 male, 4 female, mean age 17, range from 14 to 20 years. All of them $12,100 \%$ had admission x-rays and CT-scan that confirmed the presence of lumbar traumas. 10 patients had also MRI-scan (group 1), 2 patients only xrays and CT-scan (group 2). We analyzed also the type of sport activity -amateur (group a, 9 patients), professional (group b, 3 patients)-, then the timing of injury recent trauma (group I, 11 patients) and chronic trauma more than 7 days after the initial injury (group II, 1 patient).

Conclusions: Further studies were warranted but the combination of ct and mri is is very useful for the evaluation and for the therapeutic approach of spinal trauma patients.

\section{Session 2: Advances in pediatric head trauma management}

\section{PP15}

Pediatric ischemic stroke after head trauma

Patricia Barrio Fernández, Javier Pérez Suárez, Javier Ibáñez Plágaro, Javier Fernández Fernández (Leon, Spain)

Introduction: Arterial ischemic stroke occurs in approximately two or three per 100,000 children per year. Conditions such as infection, congenial heart diseases and prothrombotic disorders have been associated, but it can also appear after neck or head trauma.

Methods: This is a case report of a 7-year-old girl with a Posterior Inferior Cerebellar Artery (PICA) ischemic stroke. Clinical status, diagnostic findings, therapy, and outcome are presented and discussed with the current literature.

Results: A healthy 7-year-old girl sustained left head trauma when she was climbing stairs. She developed minimal occipital pain and vomiting and she presented to the emergency room. Without radiological exploration she was hospitalized (during two days) to neurological clinical vigilance. Twenty-two days later she presented with a sudden onset of vomiting, gait disturbance, and headache. At neurological examination the patient's cranial nerves were intact. No cerebellar dysarthria, dysmetria or dysdiadochokinesia were noted. Coagulation profile was normal, including platelet count, prothrombin time, antithrombin III, protein $\mathrm{C}$ and antilupus anticoagulant. A CT head showed an occipital fracture and an ill-defined, hypodense, non-enhancing lesion in the left cerebellar hemisphere with a slight midline shift and early hydrocephalus. MRI confirmed a left PICA territory infarct, compression of the fourth ventricle, tonsillar herniation and incipient hydrocephalus. An emergency left-sided suboccipital decompressive craniectomy was performed 
with duraplasty and resection of the necrotic cerebellar tissue. One week after the surgery, the patient reported no deficits or symptoms.

Conclusion: In children, infarction in the posterior cerebral circulation is much less commonly reported than in adults. Where a likely cause is found, head trauma is most frequent. Infarction causing cerebral or cerebellar edema in the posterior fossa may lead to coma or death, secondary to the development of obstructive hydrocephalus, tonsillar herniation and brainstem compression. Decompressive craniectomy constituted a lifesaving procedure.

\section{Session 4: Pediatric Neurosurgery and Technology}

\section{PP16}

Phenotypical characterization of microglia in the subventricular zone after neonatal hypoxia-ischemia in rat

Urs Fisch, Catherine Bregere, Laurie Chicha, Raphael Guzman (Basel, Switzerland)

Introduction: Currently, the role of microglia in the context of central nervous system (CNS) regeneration remains unclear, mainly because microglia display heterogeneous phenotypes after CNS damage. Recent studies in rat indicate that microglia accumulate in the subventricular zone (SVZ) and support neurogenesis in healthy newborn and after adult ischemic stroke. We aim to characterize the temporal profile of SVZ microglial phenotype after hypoxia-ischemia (HI) in rat neonates, and correlate it to the coexisting SVZ neurogenesis.

Methods: Postnatal day (P) 7 Sprague Dawley rats were subjected to right-sided (ipsilateral) HI or sham surgery, and sacrificed at P10, P20 or P40. Microglia were immunostained (Iba1, CD68) and quantified in the SVZ, and adjacent brain regions such as the median Corpus callosum (CC) and the parasagittal Cortex (CX).

Results: In sham animals, SVZ size and microglial density $\left(\mathrm{Iba1}^{+}\right)$remained stable between P10 and P40, but microglial activation $\left(\mathrm{CD} 68^{+} \mathrm{Iba1}^{+} / \mathrm{Iba1}^{+}\right)$which was high at $\mathrm{P} 10$, significantly declined afterwards $(p<0.0001)$.

When compared to sham SVZ, the ipsilateral SVZ in HI animals was markedly enlarged at P10 and P20 $(p<0.01)$, and SVZ microglial density significantly higher at all timepoints $(p<0.01)$. The proportion of activated microglia remained significantly elevated until P20 $(p<0.05)$.

In contrast, in the $\mathrm{CC}$ a striking difference in microglial density and activation was only noticeable at P10 $(p<0.001)$ but not later on. In the $\mathrm{CX}$, microglial density and activation were equal between sham and $\mathrm{HI}$ animals at all timepoints.

Conclusions: We observed a specific accumulation and prolonged activation of microglia in the ipsilateral SVZ after neonatal HI, which coincides with the neurogenic response after HI (reflected by the expansion of SVZ).

Additional assessment of microglia proliferation and correlation to coexisting neurogenesis as well as gene expression profiling of SVZ microglia will further clarify their role in neurogenesis during normal postnatal development, and after HI.

\section{PP17}

The iSys $1{ }^{\circledR}$ robot reduces entry and target point errors in navigation-guided frameless stereotactic implantation of depth electrodes in children with therapy-refractory epilepsy Christian Dorfer, Harald Stefanits, Georgi Minchev, Ayguel Mert, Gernot Kornreif, Stefan Wolfsberger, Thomas Czech (Vienna, Austria)

Objective: Recently, a novel technique for a navigation-guided frameless stereotactic approach for the placement of depth electrodes in epilepsy patients has been published by our group. It uses a bone fixated guide (GIDE) for stereotactic drilling, screwing of a fixation bolt and implantation of the electrode.

To further improve the accuracy of this technique, we implemented the iSys $1^{\circledast}$ robot, which avoids the need for a manual alignment of the Vertek ${ }^{\circledR}$ articulate biopsy arm along the trajectory.

Methods: Four children (3 male, 1 female) with an overall of 22 electrodes (12 left, 10 right) have been included in the study. The mean length of the planned trajectories was 48.9 $\pm 23.0 \mathrm{~mm}(21.1-97.7 \mathrm{~mm})$. All procedures were performed with the neuronavigation system Medtronic $\mathrm{S} 7^{\mathbb{B}}$ and the Synergy Cranial version 2.2 software and the iSys ${ }^{\circledR}$ robot.

Results: Digital reconstruction of the postoperative CT scans showed a mean real error at the entry point of $1.61 \mathrm{~mm} \pm 0.89$ (0.3-3.4 mm) and at the target point of $1.64 \mathrm{~mm} \pm 0.97(0.5-4.0$ $\mathrm{mm})$, which is a reduction compared to our data obtained from the manual technique of $50 \%(3.5 \pm 1.4 \mathrm{~mm}$ and $3.0 \pm 1.9 \mathrm{~mm})$. We encountered no complications. Adequate physiological recordings were obtained from all electrodes. No additional electrodes had to be implanted because of misplacement.

Conclusion: The iSys1 robot $^{\circledR}$ is an accurate, versatile and easy-to-use tool for the frameless stereotactic navigationguided placement of depth electrodes.

\section{PP18}

A new device (SecurAcath ${ }^{\circledR}$ ) to secure external CSF catheters to the skin: technical note and preliminary experience

Paolo Frassanito, Luca Massimi, Gianpiero Tamburrini, Fabrizio Pignotti, Elisabetta Peppucci, Massimo Caldarelli (Rome, Italy)

Introduction: Accidental dislocation or removal is a wellknown complication of external CSF drainage in daily clinical practice, although no data about incidence of this 
complication is available across the scientific literature so far. SecurAcath $^{\circledR}$ (Interrad Medical) is considered the standard of care for catheter securement in case of vascular access, since it provides improved catheter stability which reduces complications and total cost of patient care, in terms of decreased catheter movement or migration and subsequently decreased catheter replacement costs.

Methods: We report our preliminary experience with the use of SecurAcath ${ }^{\circledR}$ to secure CSF drainage, either ventricular or spinal, to the skin.

Results: SecurAcath ${ }^{\circledR}$ was used in 29 consecutive patients (age 3 weeks - 16 years). In particular, the device was used for 25 ventricular catheters (a patient received two catheters in the same procedure for bilateral brain abscess) and 5 spinal drainages. Period in place ranged from 1 to 4 weeks. No complication related to the use of the device was observed, in particular there was no case of dislocation or accidental removal of the catheter. The removal procedure was extremely easy. The device has proven its utility also when adjusting the length of the catheter was decided based upon radiological examination or in case of large decrease of the size of the ventricles. In fact, the cover of SecurAcath ${ }^{\circledR}$ can be easily removed, allowing to shorten the length of the catheter by gentle retraction, and then the cover is replaced. Actually, this was performed in three cases.

Conclusions: SecurAcath ${ }^{\circledR}$ is a safe and effective device to secure CSF external catheter to the skin. It is easy to applicate, stable during cleaning site, that could be completely cleaned, is easy to maintain, reduces skin surface complications, eliminates suture related needlestick injuries, remains in place for the duration of external catheter.

\section{PP19}

Intraoperative neurophysiological monitoring in pediatric patients with tethered cord and myelomeningocele

Yusuf Izci, Yunus Kaçar, Cahit Kural (Ankara, Turkey)

Object: Defect of primary neurulation that results in failure of fusion of caudal neural tube, often characterized by incomplete development of brain, spinal cord, and/or meninges. The incidence of open neural tube defects (NTDs) is occurring at a rate of approximately $0.5-1 / 1,000$ live births. Electrophysiological evaluation of the outcomes of spinal procedures is important for neurosurgeons. İntraoperative dorsal column mapping transcranial motor evoked potensials (TcMEPs), and somatosensory evoked potensials (SSEPs) have been used to assist with release the filum terminale. Release of tethered spinal cord by sectioning of the filum terminale carries a risk of injuring neighboring motor and sensory nerve roots involved in bowel and bladder control.

Methods: Eleven patients, whose ages are in between 0-3 years old, with the diagnosis of neural tube defect (cleft spine, dysraphism, meningocele, myelomeningocele, meningomyelocele) were operated assisted with the intraoperative neurophysiological monitoring techniques between 2014 and 2015. Magnetic resonance imaging was used for the preoperative diagnosis. Spinal cord release operation for tethered cord, and closure the defect operation for myelomeningocele was performed. Eight of them $(72.72 \%)$ was male and three of them $(27.27 \%)$ was female. All of them are under three years of age. Five of them $(45.45 \%)$ are new born and operation performed in three days of their life. Intraoperative neurophysiological monitoring with free-run screen showing to mapping transcranial motor evoked potensials (TcMEPs). The more recent advent and use of transcranial motor evoked potential (tcMEP) monitoring has added further information about the integrity of the corticospinal tracts including alpha motor neurons and motor spinal nerve roots. Accordingly, these IONM modalities can provide objective evidence of the extent of neural function relative to the anatomical level of a myelomeningocele.

Results: We believe that intraoperative neurophysiological monitoring is beneficial adjunt and provides that surgeons feel comfortable about the surgical field. Intraoperative neurophysiological monitoring (IONM) is used as an adjunct to prevent iatrogenic neurological injury across the entire spectrum of spinal surgeries.

\section{PP20}

Neonatal posthemorrhagic hydrocephalus ventricularsubarachnoid stenting

Oleg Volkodav, Sveta Zinchenko (Simferopol, Russia)

Pediatric neurosurgery actual task is the CSF outflow correction due to secondary post-hemorrhagic hydrocephalus combined non-traumatic (spontaneous intraventricularsubarachnoid hemorrhage) and traumatic genesis.

Material and Methods: Premature newborns posthemorrhagic hydrocephalus neurosurgical treatment method was suggested and special ventricular-subarachnoid stent (VSS) was invented and proved (Certificate in Copyright Law, pattern № 45865 from 02.10.2012). It's algorithm including subgaleal ventricular-subarachnoid drainage with following shunting via VSS to restore the physiological liquor circulation.

Results and Discussion: Thirty-five VSS operations to lowbirth-weight newborns with post-hemorrhagic hydrocephalus were performed in two modifications. Invented VSS we are used in premature newborns with minimal body mass $1180 \mathrm{gr}$ (28 weeks of gestation). In deep premature newborns with extreeme low weight (minimal newborn body mass on the moment of operation consist of 520 gr and 25 weeks of gestation) we are used the modify sylicon perforate tube from VSS. 
Post-operative quantity criteria include: neurological anamnesis, ophtalmoscopy, neuro-imaging (neurosonography, brain CT-scan and MRI).

Subgaleal ventricular-subarachnoid drainage (Certificate in Copyright Law, pattern № 34523 from 11.08.2010) via VSS supported primary effective liquor purification from blood (simultaneous ventricles and subarachnoid space) and intracranial pressure correction.

Following CSF ventricular-subarachnoid shunting (Certificate in Copyright Law, pattern № 38061, 20.04.2011) support effective liquor outflow from lateral ventricles to subarachnoid space for it physiological absorption.

Conclusion: VSS let to restore the physiological CSF outflow alternative to ventricular-peritoneal shunting with shuntdepend condition and high risk of it dysfunction and decrease time of recovery treatment.

\section{References:}

${ }^{1}$ Orlov Yu. A. Hydrocephalus. - Kiev: Health, 1995. - 87 S.

2 Papile LA, Burstein J, Burstein R, Koffler H: Incidence and evolution of subependymal and intraventricular hemorrhages; a study of infants with birth weights less than 1500 grams. J Pediatric 1978; 92: 529-534

${ }^{3}$ Volpe JJ: Neurology of the newborn. 3rd ed. Philadelphia: W.B. Saunders. 1995; XIV, 876.

${ }^{4}$ Volkodav O.V., Zinchenko S.A. Method of treatment of hydrocephalus in children //Certificate in Copyright Law, pattern № 45865, 02.10.2012., Ukraine.

5 Volkodav O.V., Zinchenko S.A. Method ventriculosubarachnoid drainage in newborns with combined intracranial hemorrhage // Copyright Law, pattern № 34523, 11.08.2010, Ukraine.

${ }^{6}$ Volkodav O.V., Zinchenko S.A. Method ventriculosubarachnoid shunt in neonates with occlusive hydrocephalus // Certificate in Copyright Law, pattern № 38061, 20.04.2011, Ukraine.

\section{PP21}

Intracerebral hemorrhages subgaleal ventricularsubarachnoid stenting in premature newborns Oleg Volkodav, Sveta Zinchenko (Simferopol, Russia)

Neonatal intra-cerebral hemorrhage high fibrinolytic activity with altered spontaneous hemostasis in the hematoma region lead sometime to it invasion with lateral ventricle and subarachnoid space communication (i.e. porencephalus).

Material and Methods: Premature newborn intracerebral hematoma surgical treatment method was suggested and proved (Copyright Law, patent № 38063, 20.04.2011). It's algorithm including porencephalus model after hematoma removal with simultaneous effective purification from blood ventricles and subarachnoid space and further physiological liquor circulation via invented ventricular-subarachnoid shunting stent (Copyright Law, patent № 45865 from 02.10.2012).

Results and Discussion: Twelve emergency neurosurgical operations were performed to the low-birth-weight newborns with intra-cerebral hematomas. Indications to the surgery are: rough hemisphere compression by the hematoma with midbrain dislocation more than $5 \mathrm{~mm}$, basal cistern deformation, homo-lateral ventricle compression with post-hemorrhagic hydrocephalus.

Neuro-imaging: neurosonography, brain CT-scan and MRI. After adequate intra-cerebral hematoma decompression special drainage from ventricular-subarachnoid stent input to hematoma cavity for external liquor purification hematoma remnant and it communication with ventricles and subarachnoid space.

Conclusion: Following ventricular-subarachnoid shunting pure liquor from the hematoma cavity after external drainage removal let to reduce the multifocal occlusive hydrocephalus risk and improve newborns rehabilitation.

References:

${ }^{1}$ Orlov Yu. A. Hydrocephalus. - Kiev: Health, 1995. - 87 S.

2 Papile LA, Burstein J, Burstein R, Koffler H: Incidence and evolution of subependymal and intraventricular hemorrhages; a study of infants with birth weights less than 1500grams. J Pediatric 1978; 92: 529-534

${ }^{3}$ Volpe JJ: Neurology of the newborn. 3rd ed. Philadelphia: W.B. Saunders. 1995;XIV, 876.

${ }^{4}$ Volkodav O.V., Zinchenko S.A. Method of the hemispheric intracerebral hemorrhage treatment in newborns // Certificate in Copyright Law, pattern № 38063, 20.04.2011, Ukraine.

${ }^{5}$ Volkodav O.V., Zinchenko S.A. Method of the hydrocephalus treatment in children //Certificate in Copyright Law, pattern № 45865, 02.10.2012, Ukraine.

\section{PP22}

Cysto-ventricular-subarachnoid stenting in newborns with benign intracranial cysts

Sveta Zinchenko, Oleg Volkodav, Larisa Korsunskaya, Seydar Abibulayev (Simferopol, Russia)

Introduction: The actual task of neonatal neurosurgery is the treatment of benign intracranial cysts (BIC) of various origins. This is the primary supra-subtentorial BIC and secondary caused by: inflammation, trauma, spontaneous intracranial hemorrhage.

Material and Methods: Method cysto-subarachnoid shunting in infants was suggested and special cystoventriculo-subarachnoid stent was invented (Certificate in Copyright Law, pattern № 46599 from 30.11.2012) with physiological liquor outflow from the BIC.

Results and Discussion: 8 cysto-ventricular-subarachnoid stenting were performed in the infants with a gestational age of 32 to 40 weeks. Indications for surgical intervention: the 
BIC progression with the liquor-hypertensive symptoms elevation, homo-lateral ventricle compression with midbrain dislocation and contra-lateral hydrocephalus, basal cisterns deformation. In 3 children multilevel hydrocephalus correction were performed due to Dandy-Walker malformation, aqueduct stenosis, cerebellar vermis agenesia with the posterior cranial fossa cyst.

It is noted that the epidermal BIC cysto-ventricularsubarachnoid stenting effectiveness was determined by their primary external sanation with subsequent internal VSS bypass.

Conclusion: Cysto-ventricular-subarachnoid stenting allowed us to unify and simplify the liquor-bypass surgery in children with BIC in the neonatal period, regardless of their origins, size, location, alternative to traditional cystoperitoneal shunting with a high risk of inflammatory complications and dysfunction of the shunt.

References:

${ }^{1}$ Orlov Yu. A. Hydrocephalus. - Kiev: Health, 1995. - 87 S.

${ }^{2}$ Volpe JJ: Neurology of the newborn. 3rd ed. Philadelphia: W.B. Saunders. 1995; XIV, 876.

${ }^{3}$ Volkodav O.V., Zinchenko S.A. Method cysto-subarachnoid shunting in children // Certificate in Copyright Law, pattern № 46599, 30.11.2012, Ukraine.

\section{PP23}

Myelomeningocele and rachischisis in premature newborns

Sveta Zinchenko, Oleg Volkodav, Larisa Korsunskaya, Seidar Abibulayev, Victor Molchanov (Simferopol, Russia)

Introduction: The actual task of neonatal neurosurgery is to improve the myelomeningocele and rachischisis - open spina bifida (OSB) treatment in premature newborns (PN).

Material and Methods: Twenty-five urgent neurosurgical operations to PN with OSB were performed, minimal newborns weight is 860 gramme, gestational age is 26 weeks; in one $\mathrm{PN}$ rachischisis Th5-L3.

OSB plastic art method in PN was proposed (Copyright №17679, 21.08.2006 and Copyright №39942, 02.09.2011), ensures minimal invasiveness, plasticity, functionality and efficiency principles.

OSB plastic art peculiarities in PN presumes extensive vertebral canal posterior wall defect closing given severe malnutrition soft tissues, rough anatomical structures failure and skin changes with CSF leak prevention.

Results and Discussion: OSB plastic method includes careful medullar plate selection by the arachnoid crease with the sac contents inspection, spinal cord untethered, skin-fascia flap formation.

Subsequent is the stored hernia sac immersion, medullar plate neurulation, vertebral canal posterior wall reconstruction. Pia-arachnoid membrane fold is formed in the vertebral canal lateral pockets to CSF absorption, spinal blood supply preserve through the pia mater vessels network. Skin-fascia flap have used for shelter - sealing of the hernia gate and vertebral canal, providing plastic reliability and CSF leak prevention.

Conclusion: This method allows to plastic OSB in PN any forms and sizes with vertebral canal posterior wall reconstruction, minimum risk of bleeding, reliable sealing in conditions of the soft tissue malnutrition, a reduction of the time of surgery and anesthesia.

\section{References:}

${ }^{1}$ Volkodav O.V., Zinchenko S.A. Method of treatment of closed and open spina bifida in premature newborns // Certificate in Copyright Law, pattern № 17679, 21.06.2006, Ukraine.

${ }^{2}$ Volkodav O.V., Zinchenko S.A. Method of two stage surgical treatment of open spina bifida in newborns // Certificate in Copyright Law, pattern № 39942, 02.09.2011, Ukraine.

\section{PP24}

Delayed hematomyelia in an expansive cervical-thoracic syrinx in a young child: another pitfall in the treatment of Chiari malformation

Clarence Jr Greene, Lori McBride, Durga Sure (New Orleans LA, USA)

Hemorrhage into a syrinx is extremely rare. Roughly once a decade a case report finds its way into the literature. Most patients who suffer the consequences of such a lesion have had trauma to the spinal cord, coagulation disorders, associated spinal cord tumors, or direct surgery in the spinal cord.

We report a 7 year old patient, who came to medical attention for scoliosis, which was secondary to Chiari I Malformation and syringomyelia. Ten months after a bony decompression coupled with dura scoring technique, she presented with sudden paraplegia secondary to hemorrhage within the syrinx.

Urgent surgical intervention with evacuation of the hematoma, and a duroplasty in the posterior fossa led to a total recovery of her motor function.

The likely mechanism of the hemorrhage is rupture of pathological vessels known to course within extensive syringes, and this child's syrinx was persistantly large following the Chiari decompression. This event calls into question whether just stabilization in syrinx size after a Chiari decompression should be considered a good outcome.

We believe our patient to be the youngest child yet reported with intrasyringal hemorrhage, and likely the first child to have such a spontaneous hemorrhage remotely in time from a Chiari decompression operation.

We alert caregivers as to the requirement of close post operative follow up and consideration of additional intervention if clear and unequivocal syrinx reduction does not occur in these patients. 


\section{PP25}

Super-selective amytal injection (Wada test) in left sided parieto-insular epilepsy

Heidrun A.M. Viltard, Silvia Vieker, Yiroslav Parpaley, Anushe Weber, Jörg Wellmer, Marec von Lehe (Bochum, Germany; Witten, Germany)

Introduction: The authors present a case of multimodal presurgical work-up including super-selective Wada testing in a 14-year old patient with pharmacoresistent epilepsy due to a focal cortical dysplasia in the left supramarginal gyrus and dorsal insula.

Methods: Preoperative 3-Tesla magnetic resonance imaging (including 3D-FLAIR and ZOOMit ${ }^{\circledR}$ sequence, susceptibility weighted imaging and angiography), Videoelectroencephalogram (EEG) and Video-EEG-based source localization were obtained. After inconclusive standard left intracarotid amytal procedure for language lateralization, a superselective Wada test via a left-sided arterial rolandic M3-segment branch of the middle cerebral artery (MCA) was additionally accomplished. Awake craniotomy was no option in this patient.

Results: Semiology and electrophysiological data were concordant with a lesion in postcentral suprasylvian area, reaching the insular cortex. During standard left internal carotid artery amytal testing (Wada) the patient suffered receptive (but not expressive) language performance deficits, hinting for an atypical language organization. During super-selective amytal injection to that artery supplying the area of the dysplasia no language deficits were obtained.

Complete lesionectomy of the involved cortical area was performed, leaving the part in white matter near the pyramidal tract behind. The pre-operative imaging was implemented in the neuronavigation data and electrophysiological monitoring was used intra-operatively. Histological results show a focal cortical dysplasia (FCD, type IIb). After a follow up of 8 weeks, the patient was seizure-free and the initial incomplete Gerstmann's syndrome and slight amnesic aphasia has resolved.

Conclusion: This case demonstrates the usefulness of superselective Wada test embedded in a multimodal imaging workup in a patient who failed the Wada testing during standard intracarotid amytal procedure.

\section{PP26}

Pathological and ultrastructural findings in focal cortical dysplasia obtained from 7 pediatric surgical cases with refractory epilepsy

Pedro Pérez-Borredá, Rebeca Conde-Sardón, Vicente Villanueva-Haba, Antonio Gutiérrez-Martín, Carlos BotellaAsunción, José Manuel García-Verdugo (Valencia, Spain)
Introduction: Focal Cortical Dysplasia (FCD) is a cortical development malformation expressed usually by partial and complex epileptic seizures along the childhood. It's a frequent pathological diagnostic after surgical resections in children with focal refractory epilepsy.

Methods: Prospective and observational study about samples obtained from the cortex of 7 pediatric patients operated since May 2014 until November 2015. Preoperatively they were studied using Electroencephalography, Magnetic Resonance, Positron Emission Tomography and Stereoelectroencephalography by depth electrodes in order to know the origin of the seizures. The surgeries were performed with Neuronavigation Medtronic StealStationS $7^{\circledR}$ and Electrocorticography. Informed consent about the study was given by the parents in all cases.

The samples were fixed by glutaraldehyde and paraformaldehyde, cut in layers of $1.5 \mu \mathrm{m}$ and $70 \mathrm{~nm}$ of thickness and observed under Optical Microscope (OM) and Transmission Electronic Microscope (TEM) Leyca Tecnai Spirit G2 ${ }^{\circledR}$ respectively. Inmunohistochemistry test allow us to differentiate Gabaergic from Glutamatergic neurons and their different synaptic buttons.

Results: The patients were 4 males and 3 females between 2 and 13 years old (median age: 7.7). The location of the FCD was frontal lobe in 4 cases and temporal lobe in 3 . The pathological results were FCD type I in 2 cases, type II in 4 and 1 pending.

Under the OM we observed cortical dyslamination in all the dysplastic samples. In some cases it's possible to see a marked reduction of astrocytes in the superficial cortical layers.

With TEM, ultrastructural abnormalities like giant dysmorphic neurons, ballon cels, fibrotic astrocytes, inclusion bodies or cel death can be seen. In some dysplastic samples we have noted a relative decrease in symmetrical inhibitory synapses.

Conclusions: It seems to be direct correlation between resected dysplastic epileptogenic zones and the histological/ ultrastructural findings under microscope. These findings differ from the control samples in all the cases.

\section{PP27}

Seizure control and clinical outcome after peri-insular hemispherotomy for refractory epilepsy in childhood and adolescence

Valentina Baro, Andrea Bartoli, Shahan Momjian, Karl Schaller (Geneva, Switzerland)

Introduction: Hemispherotomy is the treatment of choice for medically refractory epilepsy due to hemispheric diffuse epileptogenicity with acceptable functional outcome. 
Methods: Complications, seizure rate and motor function of 11 patients who underwent peri-insular hemispherotomy between 2008 and 2015 in our department were reviewed. Seizure outcome was classified according to Engel's classes. Motor skills were measured as: able to walk independently, able to walk with assistance, unable to walk.

Results: Age at seizure onset ranged from 1 day to 3 years (mean 0.5 years), age at surgery between 3 months and 21 years (mean 8.4 years). Follow-up was 0.9 to 7.5 years (mean 4.1 years). 1 patient was lost at follow-up. The underlying pathologies were: hemimegalencephaly (4 patients), perinatal vascular insult ( 3 patients), Rasmussen encephalitis ( 2 patients), Sturge Weber syndrome (1 patient) polymicrogyria (1 patient). All patients presented with hemiparesis at different severity. 3 patients underwent a previous hemispherotomy with incomplete disconnection. 7 patients were Engel's class Ia and 6 had a complete withdrawal of the anti-epileptics drugs; 1 patient was Engel's class IIb, 1 IIIa and another 1 IVb. All patients had transient post-operative worsening hemiparesis. Complications were: wound infection and one intraventricular hemorrhage requiring an external ventricular drainage. At follow-up 7 patients were able to walk without assistance, 2 patients walked with assistance and 1 was unable to walk. 6 patients were able to handle objects with the affected hand.

Conclusion: In our series, seizure control was consistent with previously reported outcomes. Patients who were not seizurefree after surgery had a severe mental retardation and behavioral disorders compatible with bilateral cerebral dysfunction. The majority of patients had good motor outcome in terms of ability to walk independently and to handle objects. Periinsular hemispherotomy is effective in the treatment of medically refractory epilepsy in selected patients with widespread hemispheric epileptogenicity.

\section{PP28}

Our first experience with intrathecal administration of baclofen to patient with Hunter syndrome

Michal Petrík, Dana Dúbravova, Vladimír Bzduch, Jarmila Hornová, František Horn (Bratislava, Slovak Republic)

Introduction: Hunter syndrome (Mucopolysaccharidosis type II) is disease with multisystemic manifestation primary treated by Enzyme replacement therapy (ERT). However ERT therapy has limited effect on spastic musculoskeletal system, which primarily affects quality of life. Patients with Hunter syndrome suffer from secondary spasticity symptoms as is progressive stiffness and contractures followed by profound loss of joint motion and tiptoe walking pattern dues to $\mathrm{m}$. gastrocnemius spasticity. Over the past several decades intrathecal baclofen is feasible method in treatment of intractable spasticity. This led to an increasing use of intrathecal baclofen in children with a number of different neurological disorders with spasticity.

Method: We used intrathecal baclofen bolus test in 10 years old boy with severe Hunter syndrome. Child has been treated since 3 years with ERT by iduronate-2-sulfatase. After positive results in fine motor functions and Modified Ashworth Scale patient was provided with programmable baclofen pump with continuous intrathecal infusion of baclofen.

Results: Approximately 4 hours after bolus test dose we observed reduction of spasticity in the upper and lower extremities, improved gait, improvement in activities involving oral motor control and speech. Modified Ashworth Scale was measured prior test with score 2-3 for upper extremity and 3-4 for lower extremity, walking on the toes, crisscrossing gait was present. In peak effect after approximately 4 hours after bolus administration of baclofen we observed reduction of spasticity in the upper and lower extremities, improved gait, improvement in activities involving oral motor control and in speech. Pump implantation was indicated and performed. Patient condition is stable on dose of $215 \mathrm{ug} / 24$ hours. Stable improvement of fine motor functions and gait, physiological straighten in joints, improvement of articulation and swallowing was achieved.

Conclusion: We present our experience with intrathecal administration of baclofen to patient with Hunter syndrome. Beneficial effects of intrathecal baclofen on quality of life have been observed and measured. Intrathecal baclofen therapy should be considered a feasible treatment for muscle spasticity in patients diagnosed with Hunter syndrome.

\section{PP29}

Focus on pediatric neurocysticercosis: about 3 cases Edouard Mazerand, Ludovic De Gentile, Sophie Gueden, Philippe Menei, Matthieu Delion (Angers, France)

Neurocysticercosis is the most frequent pediatric parasitosis of the central nervous system in the world, and its prevalence tends to grow in Europe due to migrations from endemic countries. Generally, diagnosis is made when symptoms -mainly seizures- appear. At this stage, brain imaging showing an aspect of brain abscess in patients coming from endemic countries may suggest neurocysticercosis. Positive serology combined with compatible brain imaging confirms the diagnosis, but serology may turn negative at the time the disease becomes symptomatic, as it often coincides with the death of the cysticercus. Patients may then undergo invasive procedures such as stereotactic biopsies in order to identify the parasite. This can be avoided should the diagnosis had been made at an early stage of the disease. We reviewed the cases of 3 female patients aged 10,11 and 12 year old, 2 of which being from Haitian origin, and the third one having lived 3 years in Madagascar, diagnosed with 
neurocysticercosis at Angers University Hospital (France) between 2014 and 2015. As a conclusion, we point out that the pediatric neurosurgical community should consider the value of a generalized serology for non-symptomatic children coming from endemic countries, as a non-invasive technique for the early diagnosis of cysticercosis, allowing early treatment, thus preventing the development of symptoms such as seizures.

\section{PP30}

\section{Complete endoscopic resection of a giant colloid cyst in a} child

Nida Kalyal, Jignesh Tailor, Sanjeev Bassi, Chris Chandler, Bassel Zebian (London, United Kingdom)

Introduction: Colloid cysts account for less than $1 \%$ of all intracranial tumors. They usually present in adults and are rare in children. They are slow-growing, endodermal lesions lined by columnar epithelium with mucin-secreting goblet cells and with a gelatinous centre of variable viscosity. We share our experience of endoscopically managing a giant colloid cyst in a 16-year-old boy who presented with a severe worsening headache associated with vomiting, and who was found to have a large cystic lesion (largest diameter $3.8 \mathrm{~cm}$ ) in the region of the foramina of Monro causing acute obstructive hydrocephalus.

Methods: Case report including picture and video presentations of the operative intervention and review of the literature, using PubMed.

Results: Total resection of the cyst using a purely endoscopic approach was performed with resolution of the hydrocephalus and symptoms post-operatively. Histological examination confirmed a colloid cyst. Review of the literature demonstrated that although large cysts have been reported, they are extremely rare and more so in the paediatric population with only two cysts larger than $3 \mathrm{~cm}$ previously reported - neither managed endoscopically.

Conclusion: This is the first report of an endoscopic complete resection of a giant colloid cyst in a child. It demonstrates the feasibility and safety of the approach in treating giant colloid cysts in the pediatric population.

\section{PP31}

\section{Spontaneous intracranial epidural hematoma in sickle cell} anemia: case report

Hesham Aboueleneein, Mohamed Abdelrahman, Ahmed Fouda, Ameer Assiri, Fawaz Al Hamied, Manal Ismail (Jeddah, Saudi Arabia)

Introduction: Spontaneous intracranial epidural hematoma is a rare cause of neurological deterioration in patients with sickle cell disease ${ }^{[1]}$.
Other causes of spontaneous EDH have been described in few case reports like coagulopathies ${ }^{[2]}$, paranasal sinusitis ${ }^{[3]}$, and dural vascular malformations ${ }^{[4]}$.

We report a case of a female patient who was diagnosed with EDH few hours after being admitted with a vaso-occlusive crisis. She was successfully managed by emergency craniotomy and evacuation of the hematoma.

Case Report: We report a case of a seventeen years old female patient with sickle cell anemia. She presented at first with acute severe left leg pain for which she was admitted in internal medicine department, investigated, and given intramuscular tramadol for pain control. There was no history of trauma. The conscious level started to deteriorate two hours after admission and there was no explanation for that except a possible side effect of tramadol. As the Glasgow Coma Scale had dropped to 8 , she was electively intubated and computed tomography scan of the brain was done for here. There was a big right frontal hyper dense biconvex epidural lesion suggestive of epidural hematoma with no otherwise abnormalities. Coagulation studies results were within normal limit. The neurosurgery team was urgently consulted and by that time here pupils started to be unequal; the right being more dilated than the left. An emergency right frontal craniotomy and evacuation of the hematoma was done. The skull bone was found to be abnormally bleeding. The patient was transferred to ICU postoperatively and remained there for a couple of days where she started to show neurological improvement. She was then transferred to the ward for 5 more days before she could be discharged fully conscious and neurologically intact.

Discussion: Epidural hematoma is a known potentially life threatening sequela of head trauma. It is classically described in two stages of loss of consciousness separated by a lucid interval. Few cases reports described the rare occurrence of non traumatic epidural hematoma because of various causes like coagulopathies, paranasal sinusitis and dural vascular malformations.

Sickle cell anemia, a hemoglobinopathy usually presents with vaso-occlusive crisis, was reported as a cause of spontaneous EDH. The exact pathophysiology is not yet clearly defined but various explanations have been proposed ${ }^{[5]}$.

Skull bone infarction, causing periosteal stripping and disruption of cortical bone margins, has been linked to bleeding into the epidural space. Another proposed mechanism attributes this bleeding to disruption of skull bones secondary to expanding hematopoietic tissues in response to anemia ${ }^{[5]}$.

In our reported case, no evidence of skull bone infarction was noted neither in the preoperative CT scan nor intraoperatively. After craniotomy, the skull bone was found to be abnormally thick and bleeding. We suggest the cause of this EDH to be secondary to disruption of skull bone due to expanding hematopoietic skull tissue. 
There was no evidence of infection and the bleeding profile was within normal limits.

The patient could be successfully managed by emergency craniotomy and evacuation of the EDH. Her GCS showed gradual improvement from the initial preoperative 8 to 15 two days after the operation.

Spontaneous EDH should be considered as a cause of neurological deterioration in sickle cell anemic cases and urgent management should be carried out as clinically indicated.

Conclusion: Spontaneous epidural hematoma is a rare complication in sickle cell patient which can be fatal if not expected, diagnosed and managed properly. The outcome of surgery is excellent as was the case with this reported patient.

References:

${ }^{1}$ Dahdaleh NS, Lindley TE, Kirby PA, Oya H, Howard MA 3rd. A neurosurgical crisis of sickle cell disease. J Paediat Neurosurgery 2009;4: 532-5

${ }^{2} \mathrm{Ng}$ WH, Yeo TT, Seow WT. Non-traumatic spontaneous acute epidural haematoma-report of two cases and review of the literature. J Clin Neurosci. 2004;11:791-3

${ }^{3}$ Takahashi Y, Hashimoto N, Hino A. Spontaneous epidural hematoma secondary to sphenoid sinusitis: Case report. Neurol Med Chir (Tokyo) 2010;50:399-401

${ }^{4}$ Hassan MF, Dhamija B, Palmer JD, Hilton D, Adams W. Spontaneous cranial extradural hematoma: Case report and review of literature. Neuropathology. 2009;29:480-4

${ }^{5}$ Salman, YusufA et al. 'Spontaneous Epidural Haematoma In Sickle Cell Anaemia: Case Report And Literature Review'. Journal of Surgical Technique and Case Report 4.2 (2012): 135

\section{PP32}

\section{Intracranial aspergillosis in a patient with leukemia} Yusuf Izci, Cahit Kural, Özkan Tehli (Ankara, Turkey)

Intracranial fungal infections are rare and usually presented in immunocomprised patients. Treatment is surgical evacuation of the abscess and long-term antifungal therapy. Topical antifungal agent administartion is seldom reported. In this poster, we present a case of intracranial fungal infection and treated surgically with topical antifungal administration.

17-year old male patient presented with headache, nauseavomiting and drowsiness. He had a history of acute lymphoblastic leukemia (ALL) and treatment with chemotherapy. After his admission, emergent magnetic resonance imaging (MRI) was performed and it revealed a huge left frontal contrast-enhancing mass lesion. He underwent surgical treatment with left frontal craniotomy and drainage of the frontal abscess. Intraoperative biopsy showed that this was a fungal infection (aspergillosis) and therefore we decided to insert topical antifungal agent (amphotericin-B) into the abscess cavity after drainage. The patient was improved after surgery and received antifungal therapy for 3 months. Long-term follow-up MRI confirmed the total remission of fungal infection. Children, who had the diagnosis of ALL and received chemotherapy, should be closely followed for fungal infection of the central nervous system. Prompt surgical intervention is needed in order to achieve good clinical outcome.

\section{PP33}

Early postoperative magnetic resonance imaging is not predictive for multiple intracranial abscesses in children Yusuf Izci (Ankara, Turkey)

The treatment of intracranial abscesses is surgical evacuation of the abscess associated with long-term antibiotherapy using appropriate agents. In this poster, we present 10-year old male patient admitted with nausea-vomiting and drowsiness. The magnetic resonance imaging showed multiple intracranial abscesses causing increased intracranial pressure. Emergent surgical treatment was performed and the 2 large absesses were removed with different craniotomies. Staphyloccocus aureus was detected in the abscess material and the patient underwent antibiotic administration. At the postoperative 10th day, the pediatricians performed control MRI and revealed similar contrast enhancing lesions without increased intracranial pressure. We did not suggest re-operation and followed-up the patient. Six months after surgery, the MRI confirmed total regression of all abscesses.

MRI in the early postoperative period show similar enhancements with preoperative MRI and therefore not predictive for children with multiple intracranial abscesses. Postoperative MRI should be taken at least 6 months after the surgery if the patient's clinical condition is not deteriorated.

\section{PP34}

Craniofacial bioactive bone reconstruction particularly for kids below 5 years of age

Angela Brentrup (Muenster, Germany)

In the field of neurosurgery the stuff and experience has developed during the last decades: beside the allograft bone flaps are now different deals in the neurosurgery scene, but less for kids younger than 5 years of age. Rarely severe craniocerebral injuries of small kids are survived; as well craniectomy in case of stroke is uncommen in children. Therefore the overall experience with different synthetic skull materials is little.

This study includes the need of synthetic skull implants even in very young patients, requirements for CAD/CAM skull implantations, any necessary operations before skull reconstruction, the special problems with $\mathrm{CAD} / \mathrm{CAM}$ in very young kids, the timing of operation, different avaiable skull implants and associated advantages and disadvantages. 
Finally case reports and personal conclusions for the future are presented.

\section{PP35}

Endoscopic monoportal resection of intraventricular choroid plexus papilloma in an infant

Alexander Gerard, Bassel Zebian, Jignesh Tailor, Ângela Bravo, Airi Kugisaki, Sanj Bassi, Chris Chandler (London, United Kingdom)

Introduction: Choroid plexus papillomas (CPP) are rare neuroepithelial tumours. They account for less than $1 \%$ of all intracranial neoplasms. The majority are grade I. Very few reports of endoscopic resection exist, most likely due to the highly vascular nature of the tumour and the lack of appropriate endoscopic instrumentation. We report a case of complete endoscopic monoportal resection of an atypical choroid plexus papilloma in an infant to highlight the challenges but also demonstrate the safety and feasibility.

Methods: Case report of a 5 month-old infant with an enhancing lobulated lesion within the left lateral ventricle and communicating hydrocephalus and review of the literature.

Results: Complete resection was achieved using a monoportal left parieto-oiccipital burr hole. The child developed a subdural hygroma which required a tap initially followed by delayed insertion of a ventriucloperitoneal shunt. Histologically the tumour was a grade II CPP. He made a complete recovery and is recurrence-free at 6 months.

Conclusions: Endoscopic resection of CPP in infants is safe in centres with large endoscopic experience. The main challenges to overcome are those of instrumentation and endoscopic haemostasis.

\section{PP36}

Infected cephalhematoma: case report and review of the literature

Samantha Ashworth, Airi Kugisaki, Eleni Maratos, Angela Bravo, Jose Calado, Sanj Bassi, Chris Chandler, Bassel Zebian (London, United Kingdom)

Introduction: Whilst cephalhaematomas are common birth related injuries, infected cephalhaematomas are rare and can be difficult to diagnose. We present a case of an infected cephalhaematoma in a neonate to highlight the difficulty in diagnosis and our management strategy. We also present our review of the literature.

Methods: Case report and review of the literature.

Results: Cephalhaematomas usually occur in assisted deliveries (ventouse or forceps) and are almost always managed conservatively. Reports of infected cephalhaematomas are few and far between and diagnosis can be challenging. We managed our patient by draining the infected haematoma and antibiotic administartion. The patient made an excellent recovery. Literature review supported our management strategy.

Conclusions: Although rare, infected cephalhaematomas do occur and should be considered in neonates with a cephalhamatoma when other causes of sepsis have been ruled out. Early diagnosis and treatment with drainage and antibiotic therapy ensure good outcomes.

\section{PP37}

\section{OEIS complex: case report}

Nabila Tighilt, Souad Bakhti, Youcef Benmouma, Nazim Boumahdi, Mohamed Djennas (Algiers, Algeria)

OEIS complex is a rare congenital malformation that combines omphalocele, bladder exstrophy, imperforate anus and spina bifida. Its incidence is $1 / 50000$ births. Myelomingocele is uncommonly described in this complex.

We report the case of a female newborn presenting OEIS complex with mylomeningocele associated to major hydrocephalus. The patient underwent a surgery for myelomeningocele and reservoir of Rikham placement for derivation of CSF. The patient was then managed by pediatric surgery team. A meningitis occurs in the postoperative period treated and healed with adequate antibiotics. We didn't observe skin complications. Unfortunately, the patient died from an inhalation syndrome.

Optimal timing of surgery is not well established. Some authors advocate the multidisciplinary management in the immediate postnatal period followed by abdominal reparation and meningocele closure. While for other authors, the surgery of meningocele can be postponed after healing of abdominogenital surgery.

Abdominal and genital malformations are life threatening and require then abdominal wall reparation first. However this attitude is not always done, particularly when myelomeningocele is associated because high risk of infection. According to this attitude, we have done reparation of myelomeningocele with derivation of CSF by Rickham reservoir first.

OEIS complex is a rare disease requiring a multidisciplinary surgical treatment to reduce mortality and morbidity.

\section{PP38}

\section{Spiritualism and medicine}

Ashok Kumar Mahapatra (Bhubaneswar, India)

According to the WHO, for maintaining good health, spiritualism is one dimension. Over the decades, Doctors are becoming less and less spiritual. Thus there is a need to inculcate spiritualism in medical professionals.

Spiritualism in my experience is the connection between the soul and the God. For personal development, 
spiritualism plays an important role. A spiritual doctor is likely to be more ethical as compared to a non spiritual doctor. Over the last few decades, the medical community is blamed time and again for becoming commercialized and doing unethical practice. Patients and doctors' relation is becoming more and more strain leading to dissatisfaction and lack of mutual respect. More importantly, large number of litigations is also reported for above reasons. In medical curriculum, not much humanity or ethics are taught. Thus, medical profession has become more and more materialistic. Therefore, it is imperative that doctors should have a spiritual mind. This can be done either by meditation (yoga, pranayam (breathing exercise). Hence I feel to deliver right treatment without motive; it can only be possible with development of spiritualism in health care professionals.

Conclusion: I feel it is our moral responsibility to inculcate yoga which only can be done by meditation, yoga and concentration classes. By doing so, it will be possible to improve health care delivery system and doctor and patient relation will also improve.

\section{Session 5: Oncology}

\section{PP39}

Epidemiological study of pediatric brain tumors in Ukraine before and after Chernobyl disaster

Andrii Shaverskyi, Luydmila Verbova, Leonid Marushchenko, Pavlo Plavskyi, Ivan Protsenko, Mukola Plavskyi, Andrii Svyst, Volodumur Muhaluk, Archil Agladze (Kiev, Ukraine)

Introduction: The accident at the Chernobyl nuclear power station (Ukraine) in 1986 was a tragic event for its victims, and those most affected suffered major hardship.

Objectives: We present an epidemiological study of brain tumors in children with regard to their age-relations, histology and malignancy analyzed before and after Chernobyl accident.

Material and Methods: Within 1980-2009 at the Pediatric Department of the Institute of Neurosurgery 3591 children, who had histologically verified brain tumors, were examined and treated. The epidemiological research was carried out, taking into account tumor histostructure and malignancy grade.

Results: In order of frequency the most common types were astrocytic tumors $(38.5 \%)$, embryonal tumors $(19.5 \%)$, craniopharyngioma $(10.4 \%)$ and ependymal tumors $(6.4 \%)$. Malignant tumors accounted for $45.5 \%$ of all neoplasms.

The pre-Chernobyl material (1980-1984) included 379 cases, whereas in the post-Chernobyl periods it became more sizeable: 1985-1989 -711 cases (1.9 times increase); 1990-1994 580 cases (1.5 times increase); 1995-1999 -629 cases (1.6 times increase); 2000-2004 -573 cases (1.5 times increase); 2005-2009 -717 (1.9 times increase). The analysis of brain tumor incidence in children before and after the Chernobyl disaster has clearly demonstrated its rise after the disaster. Taking into consideration the overall decline of birth (on $42.2 \%$ ) and correspondigly decrease in children's population, the 1.9 times growth of a mean incidence value seems rather substantial.

The incidence of malignant tumors (III-IV grade anaplasia) in children has also increased after Chernobyl disaster. In 1980-1984 they constituted $41.2 \%$ and in 2005-2009 came up to $46.7 \%$.

Conclusions: Analysis of brain tumor incidence in children before and after the Chernobyl disaster has clearly demonstrated its increase after the disaster. In the post-Chernobyl period malignant tumor incidence also increased.

\section{PP40}

Desmoplastic non-infantile ganglioglioma: a rare case of malignant transformation

Smith Natalie, Bassel Zebian, Bhaskar Thakur, Miren Aizpurua, Angela Calado Bravo, Safa Al-Saraj, Sanjeev Bassi, Chris Chandler (London, United Kingdom)

Introduction: Desmoplastic infantile gangliogliomas (DIG) are rare, supratentorial glioneuronal tumours of infants. They are histologically benign and considered as WHO grade I tumors. When presenting in children over the age of 1 , they are termed desmoplastic non-infantile ganglioglioma (DNIG). They are not usually associated with malignant behavior. We present a rare case of recurrent DNIG with malignant transformation.

Case Report: A four year-old girl presented with a fourmonth history of worsening headaches, aggressive behavior and left facial droop. MRI revealed a large right parietooccipital lesion with mass effect. The lesion was subtotally resected. Histology was consistent with desmoplastic infantile ganglioglioma, WHO Grade I. Follow up MRIs demonstrated aggressive growth requiring further debulking in 7 months and 10 months. The subsequent biopsies showed similar features; however increasing mitotic activity in the predominant desmin positive astrocytic cells. The proliferation index (Ki67) was focally up to $4 \%$ in the first biopsy; increasing to $8 \%$ and $12 \%$ in the subsequent biopsies. Histology from the third resection was consistent with anaplastic ganglioglioma, WHO Grade III.

Conclusion: This case of DNIG demonstrated aggressive growth despite multiple operative interventions, and histology strongly suggesting malignant transformation. Histology has shown malignant transformation, which has only been reported in a handful of cases in the literature. This has important implication for the follow up and management of these lesions. 


\section{PP41}

Anti-tumorigenic effect of galectin-3 inhibition in glioblastoma culture cells

Ricardo Santos de Oliveira, Elder Latorraca (Ribeirao Preto, Brazil)

Introduction: Glioblastomas (GB) are the most aggressive tumors of the Central Nervous System. The neoplasm exhibits a strong resistance to treatment. The actual chemotherapy anti-GB has been based in the apoptosis cell induction. Galectin-3 (gal-3) is a mammalian beta-galactoside-binding protein characterized by a shared characteristic amino acid sequence. They are expressed differentially in normal vs. neoplastic tissues and are known to play important roles in several biological processes such as cell proliferation, death and migration.

Objectives: (i) To evaluate the influence of human GB cells treatment by the cisplatin (Cis) and temozolomide (TMZ) chemotherapy in the gal-3 expression chemoresistance induction; (ii) To evaluate how the expression and subcellular location of gal-3 could influence in these cells.

Material and Methods: For this purpose, U87MG GB human cells lines were used. The gal-3 expression was genetically blocked (knockdown). To study the cellular viability the flow citometry technique was used with annexin $\mathrm{V}$ and Propidium iodide (PI) cellular staining; and to evaluate the gal-3 expression the western blotting and flow cytometry techniques were performed.

Results: Minimum inhibitory concentration for apoptosis induction and protein expression essays were $75 \mathrm{uM}$ to Cis and $1000 \mathrm{uM}$ to TMZ. The cell death studied by annexin V and PI staining showed that the gal-3 blocked cell line presented lower values to the cell death compared to the control cells (gal-3 + ) and the protein had a higher expression in cell membrane than in cytoplasm/nucleus. Conclusion: The gal-3 inhibition in GB culture cells did not potentialized the alkylating chemotherapics action.

Conclusion: The gal-3 inhibition in GB culture cells did not potentized the alkylating chemotherapics action.

\section{PP42}

From childhood to adolescence: vulnerable period in the follow up of tuberous sclerosis

Patricia Barrio Fernández, David Santamarta Gómez, Javier Pérez Suárez (Leon, Spain)

Introduction: Subependymal giant cell astrocytoma (SEGA) is the most common Central Nervous System tumor in patients with tuberous sclerosis complex (TSC). They usually occur mostly in the first two decades of life. Although these lesions are benign and non-infiltrative, they commonly arise in the region of foramen of Monro, where they can cause obstructive hydrocephalus and even sudden death.

Methods: Retrospective review of medical records of four patients with TSC and SEGA was made, highlighting the management of them in the period of transition from childhood to adolescence.

Results: Two of our patients with diagnosis of SEGA in pediatric life (6 and 12 years old, respectively), did not have brain image controls for a long period ( 9 and 12 years). Both presented with severe hydrocephalus and neurological deterioration (one of them with signs and symptoms of high intracranial pressure). Another one, with TSC and epilepsy since childhood, has not any brain MRI performed and she developed symptomatic hydrocephalus because of SEGA. These 3 cases were operated for SEGA resection.

The last patient, who has an asymptomatic SEGA without hydrocephalus, is being followed in outpatient and a brain MRI is performed every 1-2 year.

Conclusions: These findings support the recommendations proposed in the latest consensus conference on TSC (Washington, 2012). Optimal outcome is associated with early detection and treatment, so surveillance by MRI should be performed every 1-3 years in patients with TSC until the age of 25 years. Once a probable SEGA is detected, brain MRI should be performed more frequently.

As SEGA are benign lesions, a complete and safe removal whenever possible means an almost complete cure. Surgery must be considered in symptomatic SEGA, progressive ventricular enlargement and/or when there is unexplained neurological status deterioration. The presence of hydrocephalus determines worse outcome, due to its relationship with major postoperative complications.

\section{PP43}

Results of treatment of cerebellar medulloblastoma in children

Andriy Shaverskiy, Victor Morgun, Leonid Marushchenko, Ivan Protsenko, Ludmila Verbova, Ruslan Gavrish (Kiev, Ukraine)

Introduction: Cerebellar medulloblastoma is high malignant embryonic brain tumor, occurring mainly in children, characterized by early metastasis and results of treatment generally depend on the age of the child.

The purpose of this research was to evaluate the results of treatment of medulloblastoma in children of various ages.

Methods: The study was based on analysis of the results of treatment of 653 children with cerebellar medulloblastoma who were operated in Pediatric Department of Romodanov Neurosurgery Institute from 1980 to 2009. Age of children was from two weeks to 18 years. The peaks of occurrence of tumors were detected in 4 ( 84 cases) and 7 years ( 74 cases) of 
age. The localization of tumors was vermis $(81.4 \%)$ and cerebellar hemisphere (18.6\%). The tumors were solid in $97.5 \%$ cases and cystic in $2.5 \%$. Hydrocephalus was detected in 595 (91.1\%) patients.

Results: The total resection of the tumor was executed in $36.8 \%$ of patients, subtotal in $52 \%$, partial in $11.2 \%$. In $166(25.4 \%)$ cases removal was supplemented shunt operations. $49(7.5 \%)$ patients died. Catamnesis was studied in $488(74.7 \%)$ children from one month to 10 years. Surgical treatment of cerebellar medulloblastoma in children older than 3 years was supplemented by chemotherapy and radiation of all axis (protocols HIT91, HIT-2000, P-HIT 2000-BIS4, PO/02-04, SKK'92$00)$. Children up to 3 years received chemotherapy (protocol MET-HIT 2000-BIS4). The median survival was 18 months. The two-year survival rate was $42.0 \%$, five-year $6.0 \%$.

Conclusion: Children of first three years had more favorable for radical removal location of medulloblastomas (total resection was possible in $40.3 \%$ cases, subtotal in $42.0 \%$ ). The frequency of early metastasis in different age groups of children was not statistically different. The problem of correlation between volume of adjuvant therapy in different age groups of children and next survival is still unclear.

\section{PP44}

Medulloblastoma: a clinicopathological correlation of WNT/ $\beta$-catenin pathway status and clinical outcome

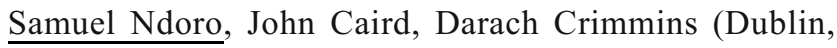
Ireland)

Objectives: The objective is to retrospectively analyze the clinical phenotypes of the 4 molecular subgroups of medulloblastoma (MB) in histologically diagnosed patients with $\mathrm{MB}$ $<16$ years of age for a 10 year period (2005-2014) and to identify which MB subgroup has the most favorable outcome in terms of overall survival time (months).

Design: Observational retrospective case series.

Subjects: Children $<16$ years of age in whom MB was histopathologically verified.

Methods: To review patient charts, histological tissue samples, radiological imaging and out-patient department follow up letters. Standard histological preparation is used to assess general architectural and cytological features. The samples are fluorescent labeled of DNA and purified. Microarray hybridization is followed by array comparative genomic hybridization processing and hybridization. Post hybridization washing, the microarray is scanned and interpreted.

Results: Immunohistochemistry plus array comparative genomic hybridization is sufficient to classify a histological sample of MB into its molecular subgroup. WNT MB accounted for $4 \%$ of our sample. Nodular desmoplastic histological subtype of MB represented $23 \%$ of results. Deceased, mean overall survival was 14.34 months.

Conclusions: A clinicopathological correlation of WNT/ $\beta$ catenin pathway and good clinical outcome exists however a large multi-centre analysis needs to be conducted.

\section{PP45}

Sinonasal adenocarcinoma involving the anterior skull base: an exceptional entity in the pediatric population

Patricia Puerta, Antonio Guillén, Franklin Santiago Mariño, Enrique Ferrer, Andrés Morales, Jordi Muchart, Mariona Suñol, Jose Luis Llorente, Laura Suárez, Mario A. Hermsen (Barcelona, Spain; Oviedo, Spain)

Objective: We present a pediatric patient with a large sinonasal non-intestinal type adenocarcinoma (non-ITAC), a rare aggressive malignancy normally seen in adults. Our aim was to outline our treatment approach and identify potential somatic or germline driver mutations.

Material and Methods: Clinical and histopathological features were analyzed. DNA was obtained from tumor and peripheral blood lymphocytes. Somatic and germline mutations were analyzed by exome sequencing and results were further studied by immunohistochemistry (IHC).

Results: A previously healthy 9-year-old male presented with frontal headaches, vomiting and behavioral changes. Physical examination showed mild left hemiparesis. Brain imaging showed a $5.9 \times 4.3 \times 5.7 \mathrm{~cm}$ right sided mass arising from the nasal cavity, eroding through the cribriform plate into the anterior cranial fossa with displacement of both frontal lobes. A combined endonasal endoscopic and bifrontal craniotomy approach achieved a gross total resection. Postoperative MRI showed no macroscopic evidence of disease. Histological examination was compatible with non-ITAC. Postoperatively, neurological symptoms disappeared and adjuvant concomitant chemo-radiotherapy was administered. The patient is in remission seven months out of therapy. Exome sequencing identified 66 somatic mutations, two of which in known cancer-related genes, AKT1 and SMARCA4. IHC analysis confirmed AKT1 pathway activation. The mutation in SMARCA4 did not result in loss of protein expression; however, the protein may be non-functional. Analysis of copy number variations from the sequencing data revealed only two chromosomal regions, $6 \mathrm{q}$ and $8 \mathrm{q}$. None of the germline mutations could be related to the disease.

Conclusion: We present the first pediatric case of a non-ITAC with intracranial involvement treated effectively with a radical surgical resection followed by adjuvant chemo-radiotherapy. Molecular analysis revealed no predisposition genetic alteration, but somatic mutations were identified in AKT1 and SMARCA4, which may play a role in tumorigenesis and should be studied for potential targeted therapy. 


\section{PP46}

Obstructive hydrocephaly caused by tumors of III ventricle in pediatric patients. Endoscopic surgery for hydrocephaly and histological verification of the tumors Volodymyr Mykhalyuk, Pavlo Plavsky (Kiev, Ukraine)

Object: Considering the efficiency of endoscopic surgery for obstructive hydrocephaly, we used simultaneously carry out histological verification of III ventricle tumors in pediatric patients.

Materials: During 2014-2015, 18 patients (age 6 months-11 years) were underwent endoscopic surgery for obstructive hydrocephaly, caused by III ventricle para-intraventricular tumors. Cystic tumors (6 cases) -performed a biopsy, cysts aspiration and implantation Ommaya reservoir into the tumor cyst cavity. Solid tumors (12 cases) performed ETV III and tumor biopsy.

Results: In all cases hydrocephaly regressed. The biopsy verified in $16(88.8 \%)$ cases. Craniopharyngioma verified in 6 cases, low-grade gliomas -in 7 cases, germinoma -in 2 cases, PNET - 1 case. In 2 cases tumor tissue was not detected.

Conclusions: Endoscopic interventions were effective for shunt-independent control for hydrocephaly in all cases. Due to histological diagnosis we determined the further treatment strategy in $88.8 \%$ cases.

\section{PP47}

Preoperative embolization as treatment adjunct of a choroid plexus carcinoma in an 8-month-old child

Dalila Forte, Amets Sagarribay Irañeta, Jaime Pamplona, Isabel Fragata, Miguel Correia, Mário Matos (Lisbon, Portugal)

Introduction: Choroid plexus tumors are uncommon neoplasms, occurring predominantly in children, representing $1 \%$ to $4 \%$ of all pediatric brain tumors. Its malignant variant, choroid plexus carcinoma (CPC) is a highly aggressive tumor, with frequent invasion of adjacent brain and associated metastasis. Although it is generally associated with a dismal prognosis, gross-total resection (GTR) is the main predictor of long-term survival. As CPC is highly vascularized, excessive bleeding recurrently limits surgical resection.

Case Report: We report a case of an 8 month-old female child presenting with increased cephalic perimeter that was diagnosed with a space-occupying temporo-parietal lesion in transfontanelar echography. She underwent brain biopsy that revealed choroid plexus carcinoma and was started on chemotherapy. After the first cycle, she developed tumor bleeding and left hemiparesis. A first surgical resection attempt was made through a temporo-parietal craniotomy, but was early aborted due to severe intra-operatory hemorrhage and failure to control the main arterial pedicles. A week after, digital subtraction angiography (DSA) was performed, with embolization of the main tumor feeders from the postero-lateral choroidal arteries. Seventy-two hours later, the tumor was totally resected, with normalization of intracranial pressure and partial recovery of left hemiparesis. An Ommaya reservoir was inserted so that intrathecal chemotherapy was administered. After 16 months follow-up the child is asymptomatic and there is no radiological evidence of recurrence in the control magnetic resonance imaging.

Conclusion: Excessive bleeding hinders operative management of choroid plexus tumors, limiting tumor removal extension and consequently long-term outcome. Preoperative embolization of choroid plexus tumors is a safe endovascular technique that reduces perioperative blood loss and allows a higher gross total resection rate. We suggest that preoperative embolization is a useful adjunct that should be considered prior to surgical resection in managing these patients.

\section{PP48}

\section{Cerebellopontine angle nerve hemangioma in a neonate}

Dalila Forte, Amets Sagarribay Irañeta, Erica Torres, Miguel Correia, Mário Matos, Carla Conceição, Eulália Calado, Manuela Mafra (Lisbon, Portugal)

Introduction: Intracranial infantile hemangiomas are extremely rare and have barely been reported in association with concurrent multiple cutaneous or visceral lesions.

Clinical Case: We report a case of a 27-day-old full term neonate who presents with a seven-day history of acute facial assymetry with progressive worsening. She showed right peripheral facial palsy and no cutaneous lesions on physical examination. Cranial magnetic resonance imaging (MRI) revealed a contrast enhancing right cerebellopontine angle (CPA) mass, with extension to the internal auditory canal. No further lesions were found on the neuroaxis exam. Right auditory evokedpotentials were absent. She was started on corticosteroids and repeated cranial MRI a few days later, showing enlargement of the lesion. A retrosigmoid approach was performed with total removal of the cisternal component of a largely vascularized lesion encasing both vestibular nerves. The postoperative course was uneventful. Histological examination demonstrated hemangioma with intralesional nerve bundles. Endothelial cells were immunopositive for GLUT1. She was started on oral propranolol and initiated a rehabilitation program. At six-month follow-up, she recovered partially and shows no radiological signs of recurrence.

Conclusion: This case encloses unique features, namely the presence of an intracranial nerve hemangioma in a neonate without associated hemangiomatosis, causing 
progressive neurological deficit. Despite their rarity, hemangiomas should be considered in the differential diagnosis of CPA lesions in children.

\section{PP49}

\section{Pediatric cerebellar glioblastoma - A case report}

Veejay Bagga, Vikky Lee, Paul Ince, Saurabh Sinha (Sheffield, United Kingdom)

Primary cerebellar glioblastoma is extremely rare in both adults and children. In adults, it accounts for $1 \%$ of all glioblastoma cases (Hur et al., 2008), whereas in children only a few cases have been described (Kulkarni et al., 1999; Kalina 2012; Reddy et al., 2013). In all cases, prognosis is poor despite surgical debulking and oncological medical therapy, with mean survival time reported to be in the region of 13 months.

Here we report a case of cerebellar glioblastoma in a 6 year old girl. In addition, we describe the long-term survival following surgical debulking and radiochemotherapy which has rarely been documented. At 24 months post-treatment, repeat MRI showed no tumour recurrence. At 30 months she was reviewed in clinic and continues to progress well without any signs of tumour progression.

\section{References:}

- Hur H, Jung S, Jung TY, Kim IY (2008). Cerebellar glioblastoma multiforme in an adult. J Korean Neurosurg Soc. 43(4):194-7.

- Kulkarni AV, Becker LE, Jay V, Armstrong DC, Drake JM (1999) Primary cerebellar glioblastomas multiforme in children. Report of four cases. J Neurosurg 90:546-550

- Kalina P. (2012) Pediatric cerebellar hemorrhagic glioblastoma multiforme. Open Neuroimag J. 6:13-5.

- Reddy GD1, Sen AN, Patel AJ, Bollo RJ, Jea A. (2013). Glioblastoma of the cerebellum in children: report of five cases and review of the literature. Childs Nerv Syst. 29(5):821-32.

\section{PP50}

The melanotic neuroectodermal tumor of infancy $-A$ rare tumor within the first year of life

Fernanda Andrade, Fernando Roberto Gondim CabralVasconcelos, Daniel Dante Cardeal, Hamilton Matushita (Sao Paolo, Brazil)

Introduction: The melanotic neuroectodermal tumor of infancy (MNTI) or melanotic progonoma is a rare and usually benign tumor with in the first year of age. There are about 480 cases described in literature since 1918. There is a predilection for the maxilla but there are also cases located in cranial vault (around 10\%). The standard treatment is complete resection. Malignant behavior has been reported in $6.5 \%$ and recurrence rate is about
$10 \%$ to $60 \%$ of MNTI. Other treatments such as chemotherapy and radiotherapy are required in these cases. Case Report: The authors present a case of 8-months-old female infant with left retroauricular mass that has grown 5 months ago. The mass was solid and fixed to the underlying skull. CT showed a expansive mass with soft interior and a thin layer of hypertrophic bone surrounding. MRI showed no intracranial invasion. This case was referred from other hospital and a small biopsy had been performed there. MNTI was the diagnosis. We performed a total excision of the tumor including its adjacent hypertrophic bone. A thin layer of normal bone was kept to avoid bone grafting.

Discussion: Embryonal tumors such as medulloblastoma, supratentorial primitive neuroectodermal tumor and atypical teratoid/rhabdoid tumor are the most common brain tumors in infants in the first year of age. These tumors are usually aggressive and have a poor prognosis. MNTI is considered a congenital neoplasm of neural crest origin, rapidly growing, and locally aggressive, although is usually benign. A rapid diagnosis and surgery are important to achieve a complete excision and have a better prognosis.

Conclusion: A close follow-up is required for at least 2 years. MNTI should be considered in differential diagnosis of benign or malignant lesions of calvarium in infants.

\section{PP51}

Frameless biopsy of diffuse intrinsic pontine glioma (DIPG) provides viable tumor cells for culture

Antonio Guillén, Nagore Gene-Olaciregui, Sonia Paco, Patricia Puerta, Mariona Suñol, Carmen de Torres, Ofelia Cruz, Jaume Mora, Andrés Morales La Madrid, Angel M. Carcaboso (Barcelona, Spain)

Introduction: The reasons behind the dramatic therapeutic failure in DIPG are not totally understood, mainly because of the lack of accurate preclinical DIPG models. Thus, we have recently established a fully translational research program in DIPG at Hospital Sant Joan de Deu Barcelona (HSJD). The main goal of such program is to develop preclinical tumor models from patient tumor biopsies and autopsies. Methods: Since April 2013 we have diagnosed nine DIPG patients at HSJD. Seven of them were biopsied using frameless stereotactic neuronavigation. Autopsy samples have been taken from six DIPG patients since May 2012. Tumor samples from biopsies and autopsies were processed and cultured following the technical approach to select DIPG stem cells in serum-free medium.

Results: All stereotactic biopsies $(\mathrm{n}=7)$ were precise and targeted tumor tissue, as confirmed by the histological and molecular analysis. All tumor samples contained viable cells that continued growing successfully in culture as tumor spheres. Only one out of six samples obtained from the 
autopsies contained viable cells in the brainstem parenchyma. Such viable tumor continues growing in culture and it was obtained from one patient treated with only three doses of upfront irinotecan-cisplatin with no previous radiotherapy, who died secondary to intratumoral bleeding one month after diagnosis. The tissue obtained from the other five autopsies belonged to patients that had received antitumoral treatment and progressed on therapy during the first or second year after diagnosis.

Conclusion: In our experience, frameless tumor biopsy reaches $100 \%$ success rate to obtain viable tumor tissue from DIPG patients. In contrast, autopsy samples do not provide viable tumor in most of the cases. Our approach has established unique preclinical research tools to study DIPG.

Funding: Fondo Alicia Pueyo, Marie Curie International Reintegration Grant (PIRG-08-GA-2010-276998), ISCIIIFEDER (CP13/00189) and Xarxa de Bancs de Tumors de Catalunya (XBTC) sponsored by Pla Director d'Oncologia de Catalunya.

\section{PP52}

\section{Tactics of thalamic tumor surgery in children}

Pavlo Plavskyi, Mykola Plavskyi, Oleksandr Voloshchuk, Archil Agladze, Gavrysh Ruslan (Kiev, Ukraine)

Introduction: Thalamic tumours account $1-5 \%$ of all brain tumours. The achievements in the field of neurovisualization and methods of surgical treatment provide more exact approach to thalamic tumours in children while minimizing surgical morbidity and postoperative invalidization.

Methods: In 2015 seven patients with thalamic tumours were treated at the Neurosurgery Institute: three boys and four girls (range 3-17 years) with gradually progressive neurological symptoms; two patients had contralateral hemiparesis of the extremities and face (central type), four had contralateral hemiparesis of the extremities and one child had tremor of the upper extremities. Preoperative magnetic resonance imaging (MRI) was conducted for all patients; tractography presented lateral location of corticospinal tract in two patients and medioventral in one patient. Tactics of surgery was defined considering the tumour and functionally important brain structures location. The anterior interhemispheric transcallosal approach was performed in four patients and one of each posterior interhemispheric transcallosal, transcortical and transsylvian approaches for tumour removal.

Results: In postoperative period all patients showed regression of brain neurological symptoms and two patients had transitoric hemiparesis. Postoperative the contrastenhanced MRI identified gross total removal in three patients, near-total and subtotal in four children, considering the complicated tumour location. Pathomorphology results were fibrillary astrocytoma Gr.II, anaplastic astrocytoma Gr.III and glioblastoma Gr.IV, and two cases each of diffuse-protoplasmic astrocytoma Gr.II and diffuse astrocytoma Gr.III.

Conclusion: Magnetic resonance tractography is essential for the choice of adequate surgical approach to thalamic tumours. Gross total or subtotal removal of thalamic tumours in children can result in a good quality of life in postoperative period.

\section{PP53}

Surgical approaches to the treatment of the tumors in infants

Albert Sufianov, Iurii Iakimov, Galina Sufianova (Tiumen, Russia)

Surgical treatment of brain tumors in infants has always been problematic for several reasons: difficulties and specific surgical procedures, the immaturity of all organs and systems in infants causes a high risk of serious complications in the intraand post-operative period.

Objective: Improve the results of surgical treatment of brain tumors in infants use different approaches.

Materials and Methods: Analysis of 12 patients under the age of 1 year (mean age 7.5 $\pm 0,9$ months.) Among the studied groups of patients were 9 boys and 3 girls. Preoperative examination included assessment of clinical and neurological status, CT, CT angiography, magnetic resonance imaging of the brain with contrast, MR tractography, MR -spectrography. According neuroimaging dominated supratentorial processes 11 cases, including 4 cases with parasellar tumors, 5 cases of tumors of the brain ventricles, 2 cases with a giant tumor of the parietal-temporal-occipital region. Subtentorial localization was represented in one case. Morphological diagnosis verification was carried out in accordance with WHO (2007), using a four histological grading, respectively grade from 1st degree (benign-Grade 1) and 4th (malignantGrade 4). Analysis of perioperative mortality was carried out depending on the timing: intraoperative (during surgery), in the early postoperative period (first 7 days) and late postoperative period (7-30 days).

Results: All patients were operated by us after admission to the hospital. In all patients we performed intraoperative ultrasound scanning, neuronavigation, neurophysiological monitoring of somatosensory, auditory, visual evoked potentials and spontaneous potentials. We have identified three groups of patients. The first group included patients with a skull base approach (cranioorbitozigomatic) and microsurgical removal of the tumors in 4 patients (parasellar tumors). The second group included 3 cases with endoscopic removal of the tumors. The third group included 5 patients operated on standard approaches (pterional transsilvian approach). 5 patients required shunt surgery due to a secondary hydrocephalus in a median of 6.5 days after surgery. According to the control magnetic resonance imaging radical removal was achieved in 8 patients, 4 patients conducted subtotal removal of the 
tumor due to a higher risk of severe neurological deficits. Histological variants were verified in $100 \%$ of cases. Of these medullobastoma, Grade IV ( $\mathrm{n}=1)$, pinealoblastoma, Grade IV $(\mathrm{n}=1)$, pilomicsoid astrocytoma, Grade II $(\mathrm{n}=1)$, gangliocytomas, Grade I ( $\mathrm{n}=1)$, ganglioglioma Grade I ( $\mathrm{n}$ $=1)$, choroid papilloma, Grade I $(n=3)$, anaplastic epindemoma Grade III $(n=2)$, ependymoma Grade II $(n=1)$, cavernous hemangioma $(\mathrm{n}=1)$.

In infants tumor supratentorial localization predominated in $92 \%$, subtentorial tumor was located in $8 \%$ of cases.

Perioperative mortality in the studied group of children missing. Complications after surgery amounted to four children with parasellar tumors who developed diabetes insipidus, requiring of a hormone therapy. Analysis of long-term results in follow-up period own research has shown that the survival rate at 1 year was $83.3 \%$ and this group consisted of children with tumors of Grade I and Grade II.

Conclusions: Surgical treatment of tumors is the primary method of treatment of brain tumors in children under 1 year, and according to our study is not associated with severe complications and mortality in perioperative period. The choice of surgical approach is determined based on the location of the tumor and does not exclude the use of skull base surgery for infans and endoscopic technique.

\section{PP54}

Pediatric brain tumors in the first three years of life: a review of 541 cases

Andrii Shaverskyi, Luydmila Verbova, Leonid Marushchenko, Pavlo Plavskyi, Ivan Protsenko, Mukola Plavskyi, Andrii Svyst, Volodumur Muhaluk, Archil Agladze (Kiev, Ukraine)

Introduction: Brain tumors in infants differ sufficiently from those of the older children in presentation, distribution and some morphological characteristics and need to be considered separately.

Methods: 541 infants (aged less than 3 years) with brain tumors were treated at the Pediatric Department of the Institute of Neurosurgery between 1980 and 2009. Those patients represented $12 \%$ of all pediatric tumor cases seen in our department during this period. From those, $49.9 \%$ tumors were supratentorial, $43.1 \%$ with lateral localization.

Results: The tumors were histologically verified in 427 $(79 \%)$ cases. In order of frequency the most common types were astrocytic tumors (all grades, 32.5\%), embryonal tumors $(22.9 \%)$, choroid plexus tumors $(10 \%)$, ependymal tumors (all grades, 14\%), craniopharyngiomas $(3.5 \%)$, germinomas $(1.1 \%)$. Malignant tumors accounted for $48 \%$ of all neoplasms. Choroid plexus papillomas were the most common tumor during the $1^{\text {st }}$ year of life $(31 \%)$.
The surgical removal of the tumor was performed in 355 (65.6\%) patients: total removal -164 , subtotal removal -115 , partial removal -76. In some cases after tumor excision, patients required CSF-shunting. 40 patients received postoperative chemotherapy. Follow-up data (from 6 months to 18 years) is available for $56.8 \%$ patients.

Conclusions: Children with brain tumors younger than 3 years of age have a worse prognosis than the older children. In their management surgical removal is the first choice of the treatment, chemotherapy does not seem to offer effective cure but can at times provide control on tumor growth. Radiation therapy should be delayed until 3 years of age.

\section{PP55}

Comparison of two isotonic solutions during brain tumor ressection in pediatric patients: a pilot-study

Mariana Lima, Iuri Neville, Sergio Cavalheiro, Patricia Dastoli, Dafne Bourguignon, Suzana Teruya, Luis Eduardo Martins, Andre Lourencatto, Bruno Deliberatto, Luiz Marcelo Malbouisson (Sao Paolo, Brazil)

Introduction: Fluid management of children during neurosurgery is not well established. Hypotonic solutions, commonly still used in pediatric patients, may enhance cerebral edema and worse operative conditions. This study compares two different isotonic solutions administered perioperatively regarding acid base and fluid electrolyte disturbances.

Methods: Children between 6 months and 10 years of age underwent elective neurosurgery for ressection of brain tumor. Patients were randomized in two groups: normal saline group (NSG) and balanced crystalloid solution group (BCSG). NSG and $\mathrm{BCSG}$ received $\mathrm{NaCl}$ $0.9 \%$ and PlasmaLyte ${ }^{\circledR}$ (Baxter) from anesthetic induction until 24 hours after surgery, respectively. Electrolytes and arterial blood gas analysis were obtained on three distinct moments: anesthetic induction (AI), immediate postoperative day (IPD), and first postoperative day (FPD).

Results: Fourteen consecutive children were randomized and included, seven on each group (NSG and BCSG). Mean age in months were 54 and 45, respectively. There was no significant variation of serum sodium on both groups $(5.42 \pm 2.87$ vs $1.42 \pm 4.43, \mathrm{p}=0.068)$ and the values were kept within normal range. Regarding the values obtained on IPD, serum chloride variation was significantly greater on NSG than BCSG $(6.57 \pm 5.42$ vs 1.33 $\pm 2.42, \mathrm{p}=0.037)$, as well as $\mathrm{pH}(-0.13 \pm 0.08$ vs -0.006 $\pm 0.095, \mathrm{p}=0.02)$ and base excess variations $(-4.98 \pm 3.12$ vs $-0.86 \pm 2.53, \mathrm{p}=0.018)$. Mean $\mathrm{pH}$ on IPD was significantly lower on NSG ( 7.25 vs $7.38, \mathrm{p}<0.01)$.

Conclusion: Serum chloride, $\mathrm{pH}$ and base excess variations were significantly higher on NSG. Although serum 
sodium variation was not statistically significant, there was a trend towards higher values on NSG. The differences between groups were more evident on the IPD with a tendency of normalization on FPD. This may be due to the higher infusion rate on the former period.

\section{PP56}

A pilocytic astrocytoma showing de novo anaplastic transformation

Mehmet Ilker Ozer, Cahit Kural, Mehmet Can Ezgu, Ozkan Tehli, Yusuf Izci (Ankara, Turkey)

Introduction: Pilocytic astrocytomas composed of \%2-6 of primary brain tumors. Most affected children ages are 8-13 years and tumor grows slowly. Mostly cerebellum, optic nerve, optic chiasma, hypotalamus and third ventricul area are get invaded. Survey without recurrence is approximately above 20 years. Transformation to malign astrocytomas is very rare, unlike diffuse astrocytomas.

Material: Nine years old male patient has come to emergency clinic with headache and vomitting. His complaints have begun three months ago but got worse for last two days. There was no loss of strength or pathological reflex at his pysical exam but papillary stasis was detected. Cystic and necrotic mass in size of $40 \times 35 \times 22 \mathrm{~mm}$ has seen at his MRI. The mass lesion pushed both thalamus and cerebral peduncles to posterior as well as fulfilled third ventricul and supracellar cystern.

Result: Patient got operated and mass was removed subtotally. Ventriculoperitoneal shunt was also inserted at same operation. Glial differentiation has been shown because of appearent fibriller ground and strong expression of GFAP at the histopathological report. The mass lesion has showed high mitotic activity and expression of Ki-67. Radyotherapy has executed after surgery. Patient has admitted to hospital again with tendency to sleep and lack of appetite after 4 months from surgery. Patient was confused and has lost place-time orientations. The tumor's size was $53 \times 40 \times 42$ which fulfilling third ventricul entirely, and it obliterated suprasellar cystern, interpeduncular system, and superior of prepontin cystern has been seen at his MRI. Surgery was performed again. Eozinofilic granuler bodies, fokal microcystic forms with partially compact fibriller areas was seen at patient histopathological examination and reported as "Pilocytic astrocytomas showing de novo anaplastic transformation" at effect of radyotherapy (Fig. 4). Patient deceased after 20 days from second surgery.

Conclusion: Malignant transformation of Pilocytic astrocytomas is extremely rare. So we share our exceptional phenomenon.

\section{PP57}

Structural features of brain tumors in children of different age groups and structural criteria for individualized treatment

Tetyana Malysheva, Pavlo Plavskyi, Andriy Svyst, Andriy Shaversky, Yuriy Orlov (Kiev, Ukraine)

Introduction: Diagnostics and treatment of brain tumours in children of different ages have special aspects and require improvement.

Objective: To identify clinicopathological features of brain tumours in children to determine the differential approach and individualized treatment.

Materials and Methods: We reviewed 3591 cases of children of different ages with histological type of tumour according to WHO criteria operated between 1980 and 2009 at the Romodanov Neurosurgery Institute (Ukraine). We had 567 children under the age of 3 years, $79.9 \%$ of them with established histological diagnosis. Lumbar liquor cytology study was performed for 855 patients.

Results: Supratentorial tumours were diagnosed in $49.6 \%$ and infratentorial in $50.4 \%$ cases. Children under the age of 3 years had $12.5 \%$ of all astrocytomas with supratentorial prevalence $(\mathrm{p}<0.01)$. Deep brain tumours of brain hemispheres $(22.5 \%)$ and lateral ventricles $(23.3 \%)$ dominate in babies. Cerebellar tumours (31.8\%$34.8 \%)$, IV ventricle (10.8\%-13.7\%) and brain stem tumours $(4 \%)$ prevail in children during the second and third years of life. Astrocytomas (Low grade) in young children were $35.9 \%$, malignant gliomas (High grade) $64.6 \%$. Supratentorial primitive neuroectodermal tumours (PNET) present $4.6 \%$ in young children and $1.4 \%$ in children of all ages. Medulloblastomas were in $17.3 \%$ and metastases were revealed in $14.9 \%$ cases at admission. Metastatic PNETs were found in $31.8 \%$ cases in young children. Postoperative mortality rate was $20.7 \%$, children with subtotal removal of the tumour died more frequently of haemorrhage in the bed of removed tumour.

\section{Conclusions:}

1. Intracranial tumours in children of young ages have features in comparison with the same indicators in the overall group of paediatric patients.

2. PNETs are complicated for morphological assessment in view of polyclonal molecular-genetic peculiarities and immature microvascular nervous system flow. The significant morphological features of diffuse astrocytoma, anaplastic ependymoma and plexus papilloma were identified in children of young age.

3. A cytological study is justified not only in cases of PNETs and ependymomas, but also other types of central nervous system tumours in order to optimize subsequent treatment. 


\section{PP58}

\section{Surgery for posterior fossa tumors in children} Jorge W.J. Bizzi, André Bedin (Porto Allegre, Brazil)

Introduction: Posterior fossa is the most common location of tumors in children. The most important prognostic factor is the complete resection for malignant and also for benign tumors. Surgery for these tumors might be a challenge, considering its proximity to brain stem, cranial nerves and major arteries and venous sinus. The purpose was to review the surgical results. Methods: Between 1996 and 2015, 374 patients, under 18 years of age, received surgical treatment for intracranial tumor and 188 were located in the posterior fossa (50\%). Data was collected prospectively, forming a database for brain tumor. Patients were operated prone, through a suboccipital craniotomy.

Results: Were operated 105 boys (55\%) and 83 girls (45\%). The most frequent presentation was intracranial hypertension in 156 patients (82\%), with hydrochepalus in 160 (85\%). The tumor location showed preference for the region of cerebellar vermis and fourth ventricle in $68 \%$ and cerebellar hemisphere in $18 \%$. Definitive treatment for hydrocephalus after tumor removal was necessary in 43 patients (27\%). The histopathology revealed astrocytic tumors in $72(39 \%)$, being $25 / 72$ pilocitic astrocytoma (25\%), embrional tumors in $70(37 \%)$, being 63/70 medulloblastoma (34\%) and ependymal tumors in $32(17 \%)$, being ependymoma in $15(8 \%)$ and anaplastic ependymoma in $15(8 \%)$. Total resection was obtained in 150 (79\%), partial resection in $32(17 \%)$ and biopsy in $6(4 \%)$. CSF leak was the most frequent complication occurring in 25 cases $(13 \%)$. The was no transoperative mortality and surgical mortality (30 days) occurred in 2 cases (1.1\%).

Conclusion: The aggressive surgical treatment aiming total resection of tumors in posterior fossa can be accomplished in most of cases with low morbidity and mortality.

\section{PP59}

Central nervous system tumors under two years of age: a single institution experience

Amets Sagarribay, Dalila Forte, Lino Fonseca, Pedro Branco, Gonçalo Novais, Miguel Correia, Mário Matos, Lissete Matos, Marta Oliveira, Sofia Nunes, Duarte Salgado, Manuela Mafra, Carla Conceição (Lisbon, Portugal)

Objective: Central nervous system (CNS) tumours at the first two years of age are uncommon, representing approximately $10-18 \%$ of all childhood CNS tumours and, despite the advances in medical and surgical care, are still associated with a poor prognosis. The specific features of this population, namely their small blood volume and its singular and diverse epidemiology, makes the treatment of this pathology a challenging issue, requiring an experienced multidisciplinary team in order to minimize risks and achieve the best results.
Methods: The authors review a surgical series of 23 cases of CNS tumours diagnosed under two years of age from January 2006 to January 2016 at Hospital Dona Estefânia (Lisbon, Portugal) and review the literature in Pubmed/MEDLINE database.

Results: Our series include 23 patients, with a slight predominance of male gender (13 males and 10 females), with a median age of 9 months, being the youngest child 13 daysold when she underwent surgery. Three of the cases were considered congenital. The majority of patients presented with intracranial hypertension signs and symptoms. Most of the tumours were located in the infra-tentorial compartment (65\%). 10 patients were submitted to only one surgical procedure and the remaining to two or more. Preoperative embolization was used in one case. There was one case of intraoperative death. Four patients were lost to follow-up. The most frequent histologic diagnosis were pilocytic astrocytoma (17\%) and anaplastic ependymoma (17\%), followed by pilomyxoid astrocytoma (13\%). There were 12 low-grade tumours and 10 high-grade tumours. Median time of follow-up was 17 months. The authors report also data about blood loss, perioperative support, complications and surgical details.

Conclusions: CNS tumours at a very young age represent a challenging subset of tumors for neurosurgeons, anaesthesiologists, intensive care staff and neuro-oncologists. A multidisciplinary approach is needed to minimize morbidity and mortality.

\section{PP60}

U-shaped dural opening for posterior fossa lesions and Chiari malformations in children

Rousinelle Da Silva Freitas, Cahit Kural, Martin U. Schuhmann (Tuebingen, Germany)

Objective: Midline suboccipital approach is the standard approach for many posterior fossa pathologies. Y-shaped dural incision is the classical technique for dura opening, however duraplasty is often necessary for watertight closure. U-shaped durotomy is an alternative with several advantages. The aim of this study was to analyze results of U-shaped durotomy in pediatric cases with median suboccipital approach.

Methods: 40 children underwent surgical treatment in the posterior fossa using U-shaped durotomy. 20 (50\%) cases had pylocytic astrocytoma, $10(25 \%)$ medulloblastoma, 5 cases $(12,5 \%)$ other tumor pathologies and 5 (12.5\%) Chiari 1 malformation. We recorded extend of exposure, necessity of dural retraction sutures, percentage of primary dura closure versus duraplasty, and rate of postoperative CSF leaks.

Result: U-shaped durotomy was easily applied in all cases. No additional lateral dural retraction was necessary for full exposure also for lateralized pathologies. The dura was closed by primary suture in $35 / 35$ tumor cases and in 1 chiari. In the 
other 4 of 5 Chiari a planned artificial graft extensionduraplasty was performed, as in one tumor case as a secondary procedure during later revision. No CSF fistula or pseudomeningocele were observed in postoperative period.

Conclusion: U-shaped dural opening can be easily applied in all pediatric posterior fossa lesions and chiari malformations. Midline or lateral seated lesions can be resected without additional dural retraction or cut. U-shaped durotomy in this series had a $0 \%$ rate of duraplasty for closure and $0 \%$ rate of CSF fistula. Compared to $\mathrm{Y}$ shape opening, $\mathrm{U}$ shape opening seems to be the superior technique.

\section{PP61}

Diffuse cerebellar swelling in high-risk medulloblastoma patients. Is posterior fossa decompression really needed? Pasquale Gallo, Kandasamy Jothy, Kaliaperumal Chandrasekhar, Drahoslav Sokol, Mark Brougham (Edinburgh, United Kingdom)

Introduction: Diffuse cerebellar swelling with upward herniation (DCSUH) in the acute post-operative period is a rare condition observed in the high-risk group medulloblastoma patients and potentially fatal in half of them. In the few previous cases described in the literature the main recommendation has been early posterior fossa decompression plus emergency chemotherapy. We report a case of DCSUH following a medulloblastoma resection managed successfully without posterior fossa decompression.

Methods: A 4 years old boy presented to our Hospital with a 2 weeks history of headache, neck pain and morning vomiting. On examination, he was neurologically intact but the presence of papilloedema on fundoscopy. Magnetic resonance imaging (MRI) showed a fourth ventricle mass with diffuse leptomeningeal metastasis and tri-ventricular hydrocephalus. He underwent gross total resection of the tumor (histopathology revealed a Large-cells Medulloblastoma). A ventriculoperitoneal shunt was placed on the 15 th postoperative day.

Results: Two weeks after the VP shunt insertion the patient developed a progressive lethargy with decerebrate posturing. MRI revealed a diffuse cerebellar swelling with upward herniation and a smaller ventricular size. High dose Dexamethasone was commenced along with emergency chemotherapy with Carboplatin and Etoposide. Three days after initiation of the treatment the patient woke up and a after a week he was back to his neurological status observed before this catastrophic event.

The DCSUH was resolved on the MRI scan performed 7 days after the chemotherapy and the leptomeningeal disease was much improved on the 2 months follow-up scan. Craniospinal radiotherapy was commenced 12 weeks after the initial tumor resection followed by another cycle of high dose chemotherapy with stem cell support.
Conclusion: The mechanisms underlying DCSUH still need to be further elucidated. However our case shows that emergency chemotherapy is the key in treatment of this condition and it shouldn't be delayed.

\section{PP62}

2 years old child with a giant low grade glioma

Ozkan Tehli, Cahit Kural, Mehmet Can Ezgu, Mehmet Ilker Ozer, Yusuf Izci (Ankara, Turkey)

Introduction: Pediatric low-grade gliomas are the most common tumors of the central nervous system in children. They encompass a heterogeneous set of tumors of different histologies. Surgery is the main therapy. Overall survival rates for patients whose tumors are completely resected are $90 \%$ or greater, 10 years from diagnosis. Combination chemotherapy or radiotherapy is recommended for the recurrent tumors.

Methods: Our patient was 2 years old. After a head trauma, the CT scan showed a giant lesion localized at the right parieto occipital region. The MRI report drew attention to that, 91x70x94 mm sized lesion was to be considered as a primitive neuroectodermal tumor or an atypical teratoid/rhabdoid tumor. The patient's EEG was normal. There was no papilledema. The only neurological deficit was ataxic walking.

Result: He was operated in our institution but the removal of the lesion was partial and had to be completed in a second intervention in order to protect the thalamostriate vein. The pathology report couldn't define the exact type of the tumor, but overall, it was a low grade glioma with ki-67 proliferation index below 1\%. 3 months after the surgery, a control MRI was performed and $51 \times 50 \times 62 \mathrm{~mm}$ sized residual tumor was found. The lesion was dissected from the normal brain tissue. The neurological examination was totally normal after the second surgery.

Conclusion: The treatment decision for children with subtotal resection has been controversial. If the likelihood of functional impairment is minimal and the neurosurgeon thinks it is feasible, a repeat surgery can be attempted to remove the residual tumor. The necrosis, which will develop after the first surgery due to the breakdown of tumor vascularity, will ease to remove the tumor.

\section{PP63}

Evaluation and management of lumbosacral myelomeningoceles in children

Cahit Kural, Mehmet Ilker Ozer, Mehmet Can Ezgu, Ozkan Tehli, Yusuf Izci (Ankara, Turkey)

Objective: Myelomeningocele are the common form of open neural tube defects that are usually associated with neurological deficits. Many techniques of repair and methods of prevention have been proposed with respect to the size of defect 
and the neurological condition of patient. The aim of this study was to report our experience on the management of lumbosacral myelomeningoceles in children.

Materials and Methods: We retrospectively analysed the data of 45 pediatric cases of surgically lumbosacral myelomeningocele treated in our department between 1998 and 2016. Twenty five (55\%) patients were female and sixteen were male, with a mean age of 4 months (ranged between 0 and 24 months). All patients had neurological deficits in the preoperative period. Computed tomography was performed in $42(93 \%)$ patients and magnetic resonance imaging in 19 $(42 \%)$ patients in the preoperative period. Repair of the myelomeningocele and closure of the skin defect were performed in all patients. The mean follow-up period was 36 months.

Results: Thirty eight (83\%) patients were operated for hydrocephalus and 12 (26\%) patients were re-operated for tethered cord syndrome during the follow-up period. Neurological worsening was not observed in any patient while cerebrospinal fluid fistula was detected in 2 patients.

Conclusion: Surgical treatment using appropriate microsurgical techniques is crucial for lumbosacral myelomeningoceles in children. Early surgical intervention with close follow-up will improve the neurological condition of the patients.

\section{PP64}

Brainstem ganglioglioma associated with status epilepticus: case report

William Gump (Louisville KY, USA)

Introduction: Gangliogliomas are known to be highly epileptogenic tumors, and most commonly occur in the temporal lobe. A small percentage arises as intrinsic brainstem tumors. The brainstem is not typically considered an epileptogenic zone.

Case Report: A 10 month old girl was hospitalized for suspected progressive seizure activity characterized by grunting, stereotyped posturing, and abnormal eye movements. Brain imaging was normal except for a small exophytic nonenhancing mass intrinsic to the right middle cerebellar peduncle. EEG was nonlocalizing. The patient progressed to refractory status epilepticus requiring continuous burst suppression. She was ultimately taken to surgery where a gross total resection of the brain lesion was achieved. Pathology was consistent with ganglioglioma.

Discussion: Postoperative imaging unfortunately also showed bilateral supratentorial hypoxic injury that may have resulted from an episode of respiratory arrest associated with prolonged severe seizure one week earlier, and at which time burst suppression had been initiated. 6 months postoperatively, the patient has global hypotonia and developmental delay, and likely cortical blindness. There has been no observed postoperative seizure activity on a regimen of lacosamide, levetiracetam, and phenobarbital. Gangliogliomas are well known to be highly epileptogenic, although they are rarely found intrinsic to the brainstem, and their potential to cause seizures in this location is not well characterized.

Conclusion: The epileptogenic potential of ganglioglioma may not be limited based on tumor location. Seizure prophylaxis should be considered with this diagnosis, independent of anatomic location. Overall long term prognosis may be favorable.

\section{PP65}

Pediatric patient with a cerebellar mass which is extended to the cervical canal

Yusuf Izci, Yunus Kaçar, Cahit Kural (Ankara, Turkey)

Introduction: Ependymoma is a tumor of the brain and spinal cord and it arises from ependymal cells these are localized at the ventricles of the brain and the centre of spinal cord. Ependymomas account for 5 to $10 \%$ of childhood brain tumors and it is the third most common posterior fossa tumor in childhood after astrocytoma and medulloblastoma/PNET. İnfratentorial ependymomas are seen about $\% 75$ in childhood. They protrude the upper servical spinal region and often grow into the shape of where they are seen. Ependymomas intercept the cerebrospinal fluid circulation and that causes hydrocephalus which is the reason for intracranial mass effect. We present our pediatric patient with a cerebellar mass which is extended to the cervical region.

Methods: Our patient was a two years old girl, whose complaints are gait disturbance for two months, and neurological findings are restricted neck movements, cerebellar disturbance and hyperactive deep tendon reflexes and grade 1 papilledema was found. MRI showed a mass in posterior fossa. A severe hydrocephalus in third and lateral ventricles was shown because of mass effect.

Result: Patient was operated in our clinic. The tumor was removed partially with suboccipital craniotomy. Histopathological findings defined an epandimoma grade two. At the fourth day after the operation we performed a CT scan and a severe hydrocephalus was seen. Therefore we made a second operation in order to insert ventriculoperitoneal shunt. After the surgery, patients' complaints and examination findings disappeared.

Conclusion: Gross total resection must be the first aim as a treatment. In spite of the development of alternative methods of treatment for ependymoma, such as chemotherapy, conventional radiation therapy and stereotactic radiosurgery, surgical treatment is still the basis of treatment procedures both in primary and recurrent tumour.

\section{PP66}

Intramedullary teratoma in a child 
Nabila Tighilt, Souad Bakhti, Fella Terkmani, Youcef Benmouma, Mohamed Djennas (Algiers, Algeria)

Teratomas of the spinal cord are very uncommon. Their occurrence in pediatric patients is rarer.

We report the case of a 6-year-old girl, who was operated for a congenital mega ureter at 3 months. She presents a limping since 6 months and a disturbance of urinary function. The neurological exam showed a spastic paraparesis, with hyperreflexia and bilateral flexor plantar reflexes and urinary incontinence.

MRI showed an intramedullary lesion extending from D7 to D11 with cystic and solid components that highly enhance after injection of contrast.

The patient underwent a surgery. Fatty tissue, bony fragments, and hair were found inside the tumor. Partial resection was performed because of the adherence of the lesion to the spinal cord.

The histological examination revealed a mature teratoma and no evidence of malignant components. There was an improvement of the neurological status in the postoperative period.3 months after surgery; the patient presented a neurological worsening. The MRI found a remnant of the solid portion without cyst. A second surgery was done with again a partial removal.

At 30 months follow-up, the patient has a slight motor deficit and a normal bowel function; she is totally independent. However she had a slight kyphosis which is now under orthopedic treatment.

Intramedullary teratoma is a very rare condition. The gold standard treatment is radical surgical excision. However some cases present tight adherences to the spinal cord and then a subtotal resection should be considered.The diagnosis is based only on intraoperative findings and the histological examination.

\section{PP67}

\section{Intraventricular schwannoma in children}

Jorge W.J. Bizzi, André Bedin, Cristina B. Bleil, Larissa B. Caetan (Porto Allegre, Brazil)

Introduction: Schwannomas represents $8 \%$ of all intracranial lesions. They occur at the cerebellopontine angle in $90 \%$ of the cases and less frequently at other location, including spine. Generally in association with a cranial or spinal nerve. The intraventricular location is not expected, because do not have any nerve in the proximity, but can occur rarely. Literature review shows only nine cases in children. The aim of this paper is to report the rare case of an intraventricular schwannoma in a teenage girl.

Case Report: N.F.B, 16 year-old presented at the emergency department with seizures. She complained of daily headaches in the last 6 months and the parents also noted gait disturbance and aggressive behavior in last few weeks. Neurological exam showed a mild left side hemiparesis and cranial vault bulging at the right parietal bone. CT scan showed an intraventricular lesion with an obstructive hydrocephalus. Cranial MRI identified a solid tumor, with heterogeneous gadolinium enhancement, measuring $6.8 \times 6.6 \times 6.6 \mathrm{~cm}$ at the right lateral ventricle atrium. The patient underwent surgery by a transparietal, transcortical approach. The tumor had a hard consistency and was very bloody. It was removed in pieces, cut with the monopolar cautery. Gross total resection was achieved and an EVD was left on the right lateral ventricle. The child had a well recovery and was discharged ten days after surgery. Pathology report revealed an intraventricular schawannoma, confimed later with immunohistochemistry techniques. After six months follow up the girl had her post-operative left leg weakness fully recovered.

Discussion: Schwannomas are rare lesions at this site of the brain, and they must be considered in differential diagnosis of intraventricular tumors. Despite being benign lesions, literature reports two cases of malignancy. Surgical resection is the main treatment, and total excision should be attempted.

\section{PP68}

\section{Suprasellar mature teratoma}

Jakob Nemir, Krešimir Saša Đurić, Marjan Rožanković, Nataša Rojnić-Putarek, Zvonka Gužvinec, Ivan Lehman, Hrvoje Jednačak (Zagreb, Croatia)

Background: Intracranial teratomas are rare and comprise about $0.5 \%$ of all intracranial tumours. Only few cases of suprasellar teratoma have been described. Although rare, hypothalamic-pituitary teratomas should be taken into account in the differential diagnosis of hypothalamic-pituitary region tumours. Our aim is to present a new rare case of suprasellar mature teratoma

Case Report: A fifteen years old female presented with frequentative headaches, cognitive disturbances and vomiting. Prior to symptoms of raised intracranial pressure she developed diabetes insipidus. Further endocrinological examination showed panhypopituitarismus. Ophtalmologic examination of eye fundus showed optical nerve edema. MSCT was performed and showed suprasellar tumor with signs of hypertensive hydrocephalus. After that, ventriculoperitoneal shunt was performed. After the patient was stabilized MRI was performed and showed the suprasellar gadolinium enhanced tumor with extension to the third ventricle. Based on the clinical status, neurological status and MRI a surgery procedure was indicated. Using frontotemporal osteoplastic craniotomy and translamina terminalis approach the tumor was removed. After surgical procedure materiala was sent to neuropathological evaluation which confirmed the diagnosis mature teratoma. Patient was after surgery transmited to the ICU and then 
to the neurosurgical pediatric department with any new neurological deficits. Control MSCT and MRI showed complete tumor removal with no neurosurgical complications.

Conclusion: Although rare, supraselar mature teratoma should be taken into account in differential diagnosis of intracranial tumors, especially in pediatric age group.

\section{PP69}

Multiple brain abscesses mimicking brain neoplasms

Alexandre Canheu, Marcio Lehmann, Antonio Dias Marcos, Luis Koury, Henrique Galles Marcos, Roberto Parente Neto, Tomas Monte Raso, Luis Penzo, Felipe Da Silva, Paulo Henrique Pires De Aguiar (Londrina, Brazil)

Introduction: Infectious diseases in infancy and childhood play an important role in the differential diagnosis of neoplasms of the central nervous system. This paper depicts a rare case of tuberculosis infection in a child mimicking metastatic disease.

Methods: Case Report: A 4 year-old boy, black, was admitted complaining of light fever and headache since the last 3 months. The CSF showed 168 white cells, lymphocytic predomination, protein $88 \mathrm{mg} \%$, glucose $5 \mathrm{mg} / \mathrm{dl}$. The tuberculin test was positive. CT scans showed multiples lesions, heterogeneous and calcified contents: right frontal and parietal, and left frontal and parietal and right occipital;, marked iodinecontrast enhancement, and also an isolated lesion with mass effect, solid-cystic contents, occupying the left portion of posterior fossa, displacing ipsilateral cerebellar hemisphere. A posterior fossa craniotomy was performed and total removal of a firm and solid encapsulated lesion with "butter-like" content was done. The pathology showed scattered areas of caseosa necrosis, and chronic granulomatous reaction. He was immediately treated with isoniazid, rifampicin and pyrazinamide. As the child woke up, he began to present clusters of generalized seizures and status epilepticus lasting two days, who ceased after inserting an intravenous midazolam line. The patient was kept inpatient one month recovering his clinical status.

Results: After 4 weeks of the discharging, the child returned with symptoms of intracranial hypertension and the images revealed hydrocephalus. A ventriculoperitonial shunt was inserted. 4 weeks after, the symptoms recurred and this time boy had been developed hypertrophic pachymeningitis. The treatment was additional corticosteroids medication. 18 months after neurological rehabilitation the boy recovered completely with normal psychomotor status.

Conclusions: Granulomatous diseases show a myriad of neurological affections that every neurosurgeon should be aware of. This difficult and rare case, call us attention how heterogeneous and multiform can be this serious disease.
Session 6: Neurovascular management

PP70

Postnatal delayed exacerbation of prenatally diagnosed dural sinus malformation

Katsuhiro Mizutani, Miwa Tomoru, Akiyama Takenori, Kanazawa Tokunori, Nagashima Hideaki, Miyakoshi Kei, Niimi Yasunari, Yoshida Kazunari (Tokyo, Japan)

Introduction: Dural Sinus Malformation (DSM) is a rare pediatric dural arteriovenous shunts disease. The knowledge of DSM has not been enough to fully understand its pathophysiology mostly because of its rarity. We experienced the prenatally diagnosed impressive DSM, whose clinical course was very unusual and was helpful for the better understanding of clinical entity of DSM.

Case Description: DSM of the male fetus was detected at 26 weeks of gestation by fetal ultra sound. Although the size of DSM once regressed at the fetal stage and the arteriovenous shunts was insignificant in neonate, the shunts drastically developed 4 months after birth. The aggravated shunts were successfully treated by the three times of endovascular transarterial embolizations.

Discussion and Conclusions: DSM of the present case changed its morphology both prenatally and postnatally and the arteriovenous shunts were drastically aggravated postnatally. Its clinical course was extremely unusual and unexpected. The repeated evaluation of shunts flow by fourdimensional Computed Tomographic Angiography was important and necessary to determine the timing of endovascular treatment. We had successfully performed endovascular treatments in proper timing and the clinical outcome of our case showed good course.

\section{PP71}

Complete resolution of Chiari malformation and syrinx after treatment of a cerebral posterior fossa arterio venous malformation: case report

Pierre-Henri Pretat, Serge Bracard, Olivier Klein (Nancy, France)

Introduction: Chiari type 1 malformation can be related to various conditions such as hydrocephalus or posterior fossa mass. We present a Chiari malformation related to a posterior fossa arterio venous malformation (AVM) associated to a large dorsal syringomyelia.

Case Report: A 14 years-old girl presented tremor episodes of the right hand with progressive worsening, resulting in writing difficulties.

Brain and spine MR highlighted a large cerebral posterior fossa AVM associated with Chiari malformation and a marked syringolmyelia from $\mathrm{C} 1$ to D9. The AVM was classified as Spetzler-Martin grade V. 
Treatment plan was: endovascular treatment (embolization, 6 courses) followed by stereotactic radiosurgery.

This treatment led to a complete radio-anatomical cure of the AVM and the resolution of both the syrinx and the Chiari malformation.

Conclusion: The best of our knowledge, we report here the fourth case $r$ about Chiari type I malformation caused by AVM.

\section{PP72}

Hemorrhagic cavernous malformations in children younger than 3 years - An increased risk?

Pedro Branco, Lino Fonseca, Gonçalo Novais, Dalila Forte, Amets Sagarribay, Miguel Correia, Mário Matos (Lisbon, Portugal)

Introduction: Non traumatic intracerebral hemorrhage corresponds to $50 \%$ of all strokes in children. Up to $57 \%$ of these hemorrhages are associated with vascular malformations, of which $10 \%$ are due to cavernomas. It is estimated that 0.05 0.15 hemorrhages/100.000 children every year are caused by cavernous malformations. Mixed adult and pediatric population studies estimate the hemorrhagic risk in 1.3-3.1\% per year, increasing to $23 \%$ during the first year after bleeding.

Methods: Retrospective analysis of all consecutive cases of intracerebral hemorrhages associated with cavernous malformation in children younger than 3 years admitted to our center between 2009 and 2014. A revision of published literature was made.

Results: Two cases were identified. Both presented with a hemorrhagic event at 2 years old, with a second hemorrhage identified imagiologically in less than 2 month. One presented with a cavernous malformation on the right motor cortex area with mild contralateral hemiparesis. The second case had a cavernoma on the inferior right cerebellar peduncle, and presented with gait instability torticollis and vomiting. Both were operated with complete resection of the lesion

Discussion: The natural history of cavernous malformations in children is not completely understood but it appears that it has an increased hemorrhagic risk comparing to the adult population. Fetal and neonates are more at risk but the risk's variation with increasing age has not been evaluated. The two presented cases show that the adult's determined risk cannot be applied in pediatric population. The presence of rebleading in both cases may represent a bleeding risk similar to the one described in fetal and neonate's cavernomas. The exact mechanism that makes this population more susceptible to a hemorrhagic event still is unknown.

Conclusion: The increase hemorrhagic risk of cavernous malformations described in nenonates may persist until latter ages, by unknown mechanisms. A better understanding of the pathology's natural history is fundamental for a better guidance and treatment decision making in this population.

\section{Session 7: Craniofacial surgery}

\section{PP73}

Temporal fascia flap and orbitoplasty for orbital reconstruction in case of severe microphthalmia

Rebeca Conde-Sardon, Eva Lopez, Patricia Gutierrez, Eloisa M.Villaverde, Pedro Perez-Borreda, Carlos Botella (Valencia, Spain)

Introduction: Microphthalmia is a rare condition which is often associated with micro-orbitism causing facial dissmetry. We report our method for orbital reconstruction for patients with severe microphthalmia.

Methods: We present three cases of severe microphthalmia, first of all, eye had previously removed by ophthalmologists and they were carrying a little eye conformer, surgery was carried by pediatric neurosurgeon and plastic surgeons. Osteotomies of the superior orbital rim and frontal bone were performed following an oval design in the frontal osteotomy, a lower strip of the removal bone was cut and taken off to increase the height of the orbit. The orbit was reconstructed repositioning the bone fragments to conform a new orbit (image osteotomies). The internal orbital coverage was provided by a temporal fascial flap from the ipsilateral side (image temporal-flap). We first designed the flap and determined the pivotal point, just under the repositioned orbital rim. We concluded the surgery with an external canthoplasty (image canthoplasty). After one week, eye conformed was located in the reconstructed orbit; this is left in the orbit for 6-8 weeks, and finally replaced for an eye prosthesis.

Results: In our patients we achieved an excellent result with an appropriate orbital size and volume, and a good coverage of the orbital cavity with temporal fascial flap.

Conclusions: There are several options to reconstruct the orbit, being the temporal fascial flap an interesting option for its features of thinness and softness, and ability of filling up the dead space and maintain the convex shape of the eye socket. Quality in orbital reconstruction is essential to achieve good cosmetic and functional results, but reconstruction options need to be all considered and choose the proper procedure based on the size, volume, shape, and area of defect, as well as the patient's features.

\section{PP74}

Parietal osteogenic distraction treatment of secondary chronic intracranial hypertension in children operated for scaphocephaly 
Gregoire Pech-Gourg, Audrey Gallucci, Nathalie Chivoret, Didier Scavarda (Marseille, France)

In the follow-up of children treated for scaphocephaly by $\mathrm{H}$ craniectomy surgery, the occurrence of a secondary coronal suture synostosis concerns about $10 \%$ of children. The occurrence of intracranial hypertension in children is exceptional but requires reoperation. We present our experience with two cases of children treated with parietal distraction.

Two children (aged 3 and 5 months) had been operated for scaphocephaly by $\mathrm{H}$ craniectomy in two different hospitals. Their clinical follow-up described partial persistence of dolichocephalic deformation and parietal stenosis. During their growth, chronic headaches have emerged with a complaint expressed at ages of 4 and 5 years. In both cases the fundus showed a significant papilledema without visual loss. The imaging assessment (CT and MRI) showed the absence of Chiari malformation and venous anomaly. We found images of parietal lobes compression related to the persistence of the temporal-parietal stenosis. An additional neurosurgical care for osteogenic distraction of a parietal-occipital flap was performed in these children.

The surgery consisted in a parietooccipital supratentorial craniotomy. Distractors (KLS Martin *) were placed so as to obtain parallel vectors upwardly and rearwardly. The distraction started at day 7 and continued at home at the rate of $1 \mathrm{~mm}$ per day. No infectious or hemorrhagic complications were noted. In one case, the distraction was stopped after 15 days and the activators were removed because of the palpation of a "stair step" on the anterior part of the distracted flap, but after checking the marked regression of papilledema at the fundus. Six months later, the fundus was normalized, the "stair step" was very much faded and did not require surgical correction at the time of removal of the distractors. In the second case, the distraction was continued up to 18 days to arrive distraction stop $(20 \mathrm{~mm})$. The fundus at 3 months was also normalized. The intracranial volumetric gains were 193.7 and 227.5 $\mathrm{cm}^{3}$.

Osteogenic distraction is an effective method of volume expansion of the skull and brain of children with complex and syndromic craniosynostosis. Its use in secondline in children operated on scaphocephaly with secondary chronic intracranial hypertension, not described so far, is possible and shows encouraging results.

\section{Session 8: Intraoperative neuromonitoring}

\section{PP75}

A single center experience in intraoperative neuromonitoring Djamila Benderbous (Cairo, Egypt)

Introduction: Since the introduction of the intraoperative neuromonitoring at El Galaa Military Hospital, the patient outcome has considerably improved and the complications decreased.

Materials: 30 cases from tethered cord, faty filum and lipome to spasticity have been operated this last fews years using intaoperative neuromonitoring assesment.

Conclusion: Since the use of the intraoperative neuromonitoring in lipoma-tethered cord and spasticity, we have improved the outcome of the patient with less complications.

\section{Session 9: Hydrocephalus}

\section{PP76}

Neuroendoscopy and optical phenomena in the Dutch golden age painting: an aesthetic and physical correlation Waleed Azab (Kuwait, Kuwait)

Introduction: The Dutch Golden Age of painting was characterized by a supreme expression of light effects and a notable application of the rules of perspective. Observing works by domestic life painters like Gerrit Duo, Gerard Ter Borch, Frans Van Mieris and Johannes Vermeer conveys a subjective impression of visual similarity between these works and neuroendoscopic views of the intracranial cavity. In this work, an attempt is made to further explain such a relation.

Materials and Methods: Neuroendoscopic video recordings and digital high resolution images of paintings by Dutch Golden Age Master painters Gerrit Duo, Gerard Ter Borch, Frans Van Mieris and Johannes Vermeer were observed to elaborate on features of similarity. The pertinent medical and art literature was then reviewed.

Results: Specific optical phenomena are shared by many paintings by the Dutch Golden Age Master painters and neuroendoscopic views of the intracranial cavity. These features peculiarly include light and color effects, as well as the rules of linear perspective.

Conclusions: The masterful depiction of light phenomena in the three dimensional spaces of the Dutch Golden Age domestic paintings creates a world of optical realism that simulates endoscopic views of the intracranial chambers. The use of an optical device, namely a camera obscura by Johannes Vermeer offers a further physical support to the presence of visual link between his paintings and the endoscopic views of the intracranial cavity. Exploring such a relation infuses our specialty with an aesthetic dimension that neurosurgery truly deserves.

PP77

Historical evolution and current surgical techniques of endoscopic choroid plexus coagulation 
Waleed Azab, Radovan Mijalcic (Kuwait, Kuwait)

Endoscopic choroid plexus coagulation (CPC) refers to a surgical treatment of hydrocephalus in which the choroid plexus is endoscopically ablated by coagulation. The evolution of the surgical technique of endoscopic CPC has a longstanding history that now spans more than one century since it was first attempted by Victor D. Lespinasse in 1910. Currently, many technical variations are successfully applied to perform the procedure. In this work we present an overview of the historical development of the surgical technique of endoscopic (CPC) until it reached its current technical status. We also present our surgical technique and nuances of (CPC).

\section{PP78}

\section{Herbal medicine today: clinical and research issues}

Mohammed Taqee Ansari (Hyderabad, India)

Demonstrate our indigenous medicines that are made by 1000 years old Herbal and Ayurvedic medicines practicing field in the spectrum of finding remedies for Asthma, Tuberculosis, Cancer and various other ailments.

The natural history, medical management, and outcome in infants with progressive posthemorrhagic hydrocephalus after intraventricular hemorrhage were studied prospectively. Infants with asymptomatic severe posthemorrhagic hydrocephalus were managed with a predetermined protocol. Outcome between groups at 1 to 2 years and at more than 3 years was compared. The natural history study, restricted to the inborn population, revealed that posthemorrhagic hydrocephalus developed in 53 of 409 infants with intraventricular hemorrhage. The progression of hydrocephalus either was arrested or regressed in 35 of 53 infants; progression to severe hydrocephalus occurred in 18 of 53 infants. The severe posthemorrhagic hydrocephalus was asymptomatic in 16 of 18 infants. The management and outcome study included both inborn and outborn infants. Of 50 infants, 12 had symptomatic severe hydrocephalus and 38 had asymptomatic severe hydrocephalus. The 16 infants managed with close observation were as likely to remain shunt free as the 22 infants managed with serial lumbar punctures. Of 38 infants, 20 were managed without shunts. At 3 to 6 years, the outcome of infants in the close observation group did not differ from that in the lumbar puncture group. Long-term outcome of infants with progression to asymptomatic severe hydrocephalus did not differ from that of infants in whom disease progression was arrested. Poor outcome in infants with intraventricular hemorrhage and subsequent posthemorrhagic hydrocephalus was related to severity of hemorrhage and gestational age at birth $<30$ weeks. Because long-term outcome of infants with severe hydrocephalus did not differ from that of infants in whom the progression of hydrocephalus was arrested or whose condition improved before hydrocephalus became severe, we currently attempt medical management of these infants.

Acknowledgement: Collaborate Company: BiotechnicaPharma.

\section{PP79}

Pediatric hydrocephalus and its treatment - An analysis of 476 cases

Elke Januschek, Nina Evertz, Andreas Röhrig, Sandra Kunze, Martina Messing-Jünger (Offenbach, Germany; Sankt Augustin, Germany)

Objective: In Europe the prevalence of congenital and infantile hydrocephalus is $0.5-0.8$ per 1000 births. After primary shunting further follow-ups become necessary during lifetime. In the first year of life the shunt failure rate can be up to $40 \%$ despite improved devices. The aim of this study was to analyze a typical pediatric hydrocephalus cohort regarding etiology, shunt types, revision rates and underlying causes.

Methods: We retrospectively evaluated all consecutive patients with congenital or infantile hydrocephalus treated by shunt implantation between 1/2010 and 12/2014 in our institution. We also captured children who were previously operated non-local or in-house before 2010 requiring a shunt revision. Hydrocephalus etiology, number and cause of revisions and shunt types/pressure levels were investigated.

Results: 476 children $(283 \mathrm{~m}, 193 \mathrm{f})$ were included. Hydrocephalus etiology was posthemorrhagic in 162 cases, dysraphic in 119, brain malformation and cystic in 79 , tumor in 28 , syndromal in 11 , posttraumatic in 11 , postinfectious in 11 , pseudotumor cerebri in 8 and other causes in 33 patients. Drainage of hygroma had to be done in 14 patients. In $46 \%$ the primary shunt insertion was non-local. In the primary in-house group a nonadjustable valve with integrated gravitational unit was implanted in $78 \%$. In 40 cases initially an antibiotic coated catheter were used. The preferred derivation was ventriculoperitoneal (84\%). $50 \%$ of the patients did not require revisions in the follow up period (1/2010-12/ 2014). No mortality and no intraoperative complications occurred. In the high risk population with posthemorrhagic hydrocephalus 2 to 5 revisions became necessary in $43 \%$.

Conclusions: Posthemorrhagic and dysraphism-associated hydrocephaluses are the most common etiologies, both with higher revision rates. Shunt surgery in early childhood is a safe procedure. The high rate of patients primarily treated in another institution demonstrates a trend to refer complicated cases to a specialized pediatric neurosurgical center. 


\section{PP80}

Pediatric hydrocephalus: factors related to shunt survival in a single institution

Sara Iglesias Moroño, Bienvenido Ros Lopez, Alvaro Martin Gallego, Antonio Carrasco Brenes, Francisca Rius Diaz, Miguel Angel Arraez Sanchez (Malaga, Spain)

Introduction: Shunting for hydrocephalus is the most frequent procedure in Pediatric Neurosurgery, being revision surgery needed several times along the life of the same patient. Improve shunt survival and know factors related to failure, are still a challenge for neurosurgeons, in order to avoid associated morbidity.

The aims of this study were analyze shunt survival in a long time follow up series in a single institution and assess the associated factors.

Methods: A retrospective review of 166 pediatric patients diagnosed of hydrocephalus who underwent 425 shunt procedures between 2000-2015 was conducted. Patient characteristics as age, hydrocephalus etiology, meningitis; surgical procedure: burr hole location, type of first valve and catheters; and complications: hemorrhage, shunt infection and overdrainage were studied. Statistical analyses were done using chisquare, Kaplan-Meier survival curve, and multivariate analyses.

Results: During 93 months of mean follow-up, 259 shunt revisions were performed, with $60 \%, 40 \%$ and $25 \%$ shunt survival rate at 2-, 5- and 10-year followup. Post hemorrhagic and post infectious hydrocephalus, meningitis episode, posterior burr hole location, differential pressure valve and standard catheters at first surgery and symptomatic overdrainage were statistically significant related to failure. In the multivariable analysis, previous meningitis and symptomatic overdrainage were risk factors for shunt failure and frontal burr hole location and antibiotic impregnated catheter type were protective.

Conclusion: Despite certain limitations and biases, the more important predictors of shunt failure in multivariable analysis were meningitis and symptomatic overdrainage. Frontal burr hole and antibiotic impregnated catheters at first surgery were associated with better shunt survival. Prospective, randomized, and controlled trials are required to validate these results.

\section{PP81}

Management of complex hydrocephalus with the help of telemetric shunt reservoir pressure readings - Challenges and future strategies. A case study

John Preston, Priyank Sinha, John McMullan, Hesham Zaki, Shungu Ushewokunze, Saurabh Sinha (Sheffield, United Kingdom)
An evaluation of the first six months after insertion of a Miethke sensor reservoir ${ }^{\circledR}$ into a 3 year old boy with complex hydrocephalus, using pressure readings to guide adjustments to a combined proGAV ${ }^{\circledR}$ and proSA ${ }^{\circledR}$ ventriculoperitoneal shunt system.

Management of complex hydrocephalus in children can be challenging and can present diagnostic dilemmas. We replaced a standard reservoir of an indwelling proGAV/ proSA $^{\circledR}$ combined ventriculoperitoneal shunt system with a Miethke sensor reservoir ${ }^{\circledR}$ in a 3 year old boy with complex hydrocephalus. The child had undergone numerous neurosurgical interventions including shunt revisions, extraventricular drains and ICP monitoring. By combining telemetric pressure sensor readings along with reported symptoms we were able to guide his management without the need for further surgical intervention.

We acknowledge the limitations of our study, namely that we currently only have two months of data; although we will have 6 months of data at the time of presentation. However this is a pilot study evaluating the use of the Miethke sensor reservoir ${ }^{\circledR}$ in pediatrics and our initial results have been encouraging. We will present data from the pressure sensor alongside symptoms reported and discuss our experience of its use in this pediatric case study.

We believe the use of this device can improve the management and reduce the need for surgical intervention in children with complex and difficult to manage hydrocephalus.

\section{PP82}

Slit ventricle syndrome: diagnosis and clinical management

Angelo Silva Neto, Cesar Garcia, Glena Marcelino, Vanessa Almeida, Daniel Ferreira (Natal, Brazil)

Introduction: Cerebrospinal fluid hyper-drainage may be associated with a neurological syndrome characterized by intense headache that interferes with the quality of life of patients with a ventriculoperitoneal shunt. When the findings are associated with the image of slit ventricles, it is known as Slit Ventricle Syndrome (SVS).

Objectives: Make a brief review and report the experience of six cases presenting SVS that were treated surgically.

Method: The collection of cases relating the hyper-drainage theme and the SVS. Review of the literature.

Results: Among the patients evaluated, ages ranged from 4 to 14 years, the causes of hydrocephalus were: meningitis sequelae (1), prematurity (2), Aqueductal stenosis (1) and Chiari (2). The main symptom was headache in $83.3 \%$; nausea and irritability in $66.6 \%$ of cases. Among the patients with headache, 2 had symptoms of intracranial hypotension.

Scaphocephaly was seen in 2 cases. Intracranial pressure was monitored for 48 hours in 3 patients, with a high ICP confirmation (above $20 \mathrm{mmHg}$ ) in 2 , both at night. In one case there 
was an oculomor nerve palsy due to the poor positioning of the ventricular catheter. The treatment was the shunt revision in all cases. In 3 of them, an anti-siphon programmable valve devices was chosen, and in the others it was the exchange of a low-pressure for a medium pressure system. In 2 patients, both with cranio-cephalic disproportion, the symptoms remained and a bitemporal craniotomy was needed for cranial expansion with dramatic response.

Conclusion: Most neurosurgeons that treat children with shunts find patients with SVS and shall take appropriate therapeutic decisions. The intracranial pressure monitoring, the review of the shunt valves with anti-siphon mechanism, and the adjustment of the pressure gradient usually solve most of the cases. In rare situations, often with evident cranio-cephalic disproportion, a craniotomy is required and the outcome is proper.

\section{PP83}

Semiology of dysfunction of ventriculo-peritoneal shunting in children $-A$ review

Estelle Neiter, Catherine Guarneri, Pierre-Henri Pretat, Anthony Joud, Jean-Claude Marchal, Olivier Klein (Nancy, France; Rennes, France)

Introduction: Ventriculo peritoneal shunting (VPS) is a treatment of hydrocephalus that may dysfunction. The clinical presentation of shunt dysfunction is variable. We therefore decided to focus on clinical presentation of VPS malfunction in children, for this condition requires an emergency treatment and because of the sometimes confusing signs of intracranial hypertension in a shunted child.

Materials and Methods: We searched in PubMed (PubMed (US National Library of Medicine; National Institutes of Health; http://www.ncbi.nlm.nih.gov/pubmed) with the following groups of keywords: (dysfunction OR blockage) AND shunting AND hydrocephalus; shunt complications AND hydrocephalus; hydrocephalus AND shunt AND malfunction. Articles dealing with ventriculo atrial shunt were excluded.

79 articles were retained for analysis (English and French). Case reports were excluded.

Results: The clinical presentation is variable by age: vomiting and alterated level of consciousness are the most frequent signs in older children, whereas infants present more often with raised intracranial pressure symptoms such as nausea, vomiting, irritability and bulging fontanel. Drowsiness is a good predictor of VPS dysfunction. An asymptomatic presentation is rare but possible. An abdominal presentation is also possible, ranging from abdominal discomfort to peritonitis. Fever, short time since the last intervention and irritability are good predictors of shunt infection. Pumping the chamber of the VPS has a weak positive predictive value (12\%). Shunt dysfunction can lead to death, with an estimated mortality rate at $1 \%$ per year during the first years.

Conclusion: It is essential to be aware of the variability of clinical presentation of VPS dysfunction, because of the potential severity of this condition. It is important to pay attention to the comments of the parents, especially if the child experienced a previous shunt malfunction.

\section{PP84}

Intradiploic growing skull fracture: review of mechanisms and literature

Luc Le Fournier, Pierre-Louis Henaux, Claire Haegelen, Maia Proisy, Laurent Riffaud (Rennes, France)

Purpose: A rare case of intradiploic growing fracture of the occipital bone is presented. Examination of this case and literature review was carried about to find significant specific features of such a rare condition.

Case: A 4-year-old boy was operated on for a huge occipital intradiploic growing skull fracture. His medical history, surgical management, and postoperative course are presented.

Literature Review: A computer literature search using the Medline database revealed 31 well-documented cases.

Conclusions: Special attention must be paid to skull fractures in childhood when occurring near CSF large spaces, especially if ventricle enlargement, suggesting that hydrocephalus is present. Surgeons should evaluate hydrocephalus before treating such lesions in order to improve surgical result and postoperative clinical evolution.

\section{PP85}

Bedside-implanted percutaneous-tunneled transfontanellar external ventricular drainage (PTTEVD) in the management of hydrocephalus in extremely low birth weight infants

Mino Zucchelli, Mariella Lefosse, Francesca Nicolini, Ercole Galassi (Bologna, Italy)

Introduction: Hydrocephalus treatment in extremely low birth weight (ELBW) infants still represents a challenge for both the pediatric neurosurgeon and the neonatologist, even more when the weight of the patient is far below 1,000 grams. In these cases, the benefits of an early treatment do not easily coincide with the safety of the cure, especially considering the great difference in surgical risk before the weight increases.

Methods: Ten cases of posthemorrhagic hydrocephalus (PHH) in ELBW infants (half of cases $<700$ grams, range 550-1000 grams) were treated with a percutaneoustunneled transfontanellar external ventricular drain (PTTEVDr) implanted at bedside as a first measure of a stepwise approach. PTTEVDr remained in place for an average of 24 days (range $8-45$ days). The average 
duration of the procedure was 7 minutes, blood loss was absent.

Results: In all cases early control of the hydrocephalus was achieved. One case had a single episode of CSF leakage (due to insufficient CSF removal), in another case enterococcus in the CSF sample was detected the day after abdominal surgery with ileostomy (infection resolved with intrathecal vancomycin instillation), and one case died of streptococcus sepsis (systemic infection existing prior to PTTEVD placement). Once reached $1 \mathrm{~kg}$ of weight, when necessary, the ventriculo-peritoneal shunt was implanted and the PTTEVD removed.

Conclusion: After the introduction of the PTTEVD, the needs of these babies were cared for quickly and, overall, with less hassle for both the neurosurgeon and the neonatologist: the introduction of the -only apparently- simple technique in our standard protocol of management of PHH has proved to be a wise option for those small patients.

\section{PP86}

Intra-peritoneal sheath formation after ventriculoperitoneal shunt in a patient with Shprintzen-Goldberg syndrome

Mihoko Kato, Sachiko Kato, Daimon Shiraishi, Hirokatsu Osawa (Aichi, Japan)

During ventriculo-peritoneal shunt (VPS) revision we often find a subcutaneous connective tissue sheath that surrounds a peritoneal catheter. However an intra-peritoneal connective tissue sheath is rare. We present a case associated with shunt malfunction caused by an intra-peritoneal connective tissue sheath. We tried to analyze mechanism of the sheath formation.

Case: A 5-year-old girl had a right V-P shunt due to congenital hydrocephalus 6 months after birth. She was diagnosed Shprintzen-Goldberg syndrome at 3 years old. She was admitted with vomiting and abdominal distention. We immediately changed VPS to external ventricular drainage (EVD). After the procedure, she showed persistent abdominal distension and finally received laparotomy due to ileus. During operation, we found a connective tissue sheath in peritoneal space. The sheath was located from the abdominal wall to the mesentery and created an ileus band. We also found an ulcer on mesentery caused by her old peritoneal catheter.

Discussion: The ileus caused by peritoneal catheter was previously reported. In such condition, patients usually recovered by EVD. However, the present case was not improved by simply pulling out the catheter, because a connective tissue sheath in the peritoneal space was created. It is unclear whether the Shprintzen-Goldberg syndrome is involved in the connective tissue sheath formation or not. The sheath had two portions. One consisted of the connective tissue and another consisted of the omentum. This rare condition could be created by a connective tissue sheath and omentum adhesion.

Conclusion: In shunt malfunction with persistent ileus, we should consider a sheath formation in the peritoneal space.

\section{PP87}

Symptomatic spinal arachnoid cysts in pediatric patients Miroslav Gjurasin, Ana Tripalo Batos, Vlasta Djuranovic, Pavle Miklic, Tonci Grmoja, Ljiljana Popovic (Zagreb, Croatia)

Introduction: Spinal arachnoid cysts in pediatric patients are rare. The cyst may be intradural or extradural. If sypmtomatic cyst causes a progressive neurologic deficit, most often weakness of lower extremities, neurosurgical treatment is mandatory. Methods: In this work we present 2 symptomatic pediatric patients with spinal arachnoid cyst, one intradurally and other extradurally located, surgically treated at Children's Hospital Zagreb - Klaiceva. We analyze clinical presentation, radiologic and operative findings, and outcomes.

\section{Results:}

Case 1: A 19-months-old boy was hospitalized due to progressive leg weakness. Spinal MR showed intradural dorsal arachnoid cyst at the Th3-Th9 level, with significant medullary compression. Osteoplastic laminotomy and removal of the cyst was performed. At operation, a well encapsulated tensive intradural cyst with bright fluid inside the thick wall was fenestrated and removed. Histology confirmed the arachnoid cyst. After rapid postoperative improvement of neurological deficit, the patient completely recovered during 2 -months period of rehabilitation.

Case 2: An 11-years-old girl presented with 2 weeks history of back pain, difficult walk and significant lower extremity weakness progressed to severe paraparesis. Spinal MR showed large dorsal spinal epidural cystic formation at the Th12-L2 level, enlargement of spinal canal and neural foramina, and significant compression and anterior dislocation on neural structures including medullary conus and neural roots. At operation, after osteoplastic laminotomy and opening of the widely exposed brightfluid cyst, at the bottom of the cyst there was a mediosagital cleft of the dura with multilayer arachnoid passing through this cleft and forming the epidural arachnoid cyst. The dural cleft was primarily sutured in a regular fashion. After 5-months period of rehabilitation, the recovery was complete.

Conclusion: In symptomatic patients, especially with progressive neurologic deficit, operative removal of the cyst has to be performed as soon as possible. 


\section{PP88}

The isolated fourth ventricle: surgical treatment. Review of the literature and a report of six cases

Angelo Silva Neto, Cesar Garcia, Glena Marcelino, Vanessa Almeida, Daniel Ferreira (Natal, Brazil)

Introduction: Patients with hydrocephalus that develop infection, ventricular hemorrhage or shunt overdrainage may present Isolated Fourth Ventricle (IFV) from the ventricular system. The resultant mass effect may induce a compression of the brainstem. The correct diagnosis of this condition is challenging.

Objectives: Review of the literature and report six cases that presented IFV and were treated.

Method: The collection of medical records of patients that presented IFV.

Results: Of 06 patients, all underwent neuroendoscopic procedures. Ages ranged from 18 months to 32 years. The average follow-up is 37.6 months (11-60). The most frequent symptoms were somnolence $(83.3 \%)$, followed by feeding difficulty $(50 \%)$ and headache $(66.6 \%)$. 2 cases had neurocysticercosis (NCC), 2 patients presented DandyWalker malformation with hyper-drainage (DWH), and 4 cases were posthemorrhagic hydrocephalus of prematurity (PH). During follow-up, 1 case of $\mathrm{PH}$ died from infectious complications. Aquedutoplasty was perfo rmed in 5 cases, and in three cases we used the neuronavigation due to the large abnormality and septations of the ventricular system. In cases with NCC, we removed the cysticercus and made thirdventriculostomy. In one of the patients with $\mathrm{PH}$ we performed inter-ventriculostomy communicating the third ventricle with the fourth ventricle, through the posterior wall of the third ventricle. With the exception of two cases presenting NCC, all patients had shunt (VP). In two cases there was a re-operation for put a stent in the aqueduct, using a multifenestrated ventricular catheter and connecting to the shunt. Such an approach was employed directly in other cases of endoscopic aquedutoplasty. There was partial improvement of symptoms in 2 cases and complete remission in the rest.

Conclusion: In patients with isolated IV ventricle, prompt recognition of the signs and symptoms and the appropriate neuroendoscopic planning allows good surgical results. The aquedutoplasty with interposition of stent, and aid of neuronavigation, proves to be the treatment with better results.

\section{PP89}

Combined technique (endoscopic and shunting) for the treatment of posthemorrhagic hydrocephalus in children Yusuf Izci, Cahit Kural, Özkan Tehli (Ankara, Turkey)

Posthemorrhagic hydrocephalus in children is an important clinical problem for neurosurgeons. Endoscopic third ventriculostomies are usually failed and ventriculoperitoneal shunts are obstructed frequently. A total of 3 pediatric patients underwent surgery for posthemorrhagic hydrocephalus between 2008 and 2012. The mean age was 22 months (ranged between 3 months and 15 years) and 2 patients were male and one patient was female. Germinal matrix hemorrhage was the cause of hydrocephalus in 2 patients and head trauma in one patient. Endoscopic third ventriculostomy+shunting was performed in 2 patients and endoscopic septostomy+shunting in one patient. Clinical improvement was observed and the size of lateral ventricles was diminished in all of 3 patients. The opening of the intraventricular adhesions and thick membranes were main difficulties of the endoscopic surgery while the main advantage of endoscopy is the correct placement of the ventricular catheter of the shunt. Yellow and irregular ventricular surface caused poor vision and orientation during endoscopy. No shunt revision was performed in these patients. Children with posthemorrhagic hydrocephalus are poor candidates for endoscopic treatment. Intraventricular adhesions and thick membranes are the major problems during surgery. Anatomical variations and yellow ventricular surface deteriorate the orientation of neurosurgeon. Combined technique including endoscopic fenestration and ventriculoperitoneal shunting seems the best approach for posthemorrhagic hydrocephalus in children.

\section{PP90}

The invisible cyst: triventriculomegaly due to third ventricular ependymal cyst

Anand Pandit, Mahum Imran, Nida Kalyal, Jozef Jarosz, Anastasia Gontsarova, Istvan Bodi, Andrew King, Sanj Bassi, Chandler Chris, Bassel Zebian (London, United Kingdom)

Introduction: Ependymal cysts are neuroepithelial cysts with histological similarities to choroid plexus cysts. They rarely occur in the third ventricle and, even more rarely, cause obstructive hydrocephalus in this location. We describe an ependymal cyst of the posterior third ventricle in a 13-year old child with tinnitus, delayed puberty and triventricular hydrocephalus identified on conventional MRI sequences. The cyst was only clearly visible once high resolution mid-sagittal CISS MRI was performed. The child underwent endoscopic resection with significant improvement in both outcome and radiological appearances post-operatively.

Method: Case report with pictures and video of the operative intervention as well as discussion of the histology and review of the literature.

Results: Complete resection was achieved using a monoportal image-guided endoscopic approach. We also performed a third ventriculostomy to prevent future adhesion formation at the level of the aqueduct. Histologically, the cyst was 
consistent with a benign epithelial cyst. The lack of colloid material favoured the diagnosis of ependymal cyst. Clinically, there was almost complete resolution of her tinnitus following the operative intervention.

Conclusion: Ependymal cysts of the third ventricle can rarely cause obstructive hydrocephalus and can be difficult to detect on routine imaging. We recommend that in cases of obstructive hydrocephalus with no clear cause, high resolution T2-weighted MRI sequences such as CISS or FIESTA are carried out. When a cyst is identified, our preference is for a monoportal endoscopic approach to resection, if this is deemed safe intra-operatively.

\section{PP91}

Use of gravitational shunts in the treatment of pediatric hydrocephalus. A single centre experience

Christos Chamilos, Spyros Sgouros (Athens, Greece)

Introduction: We describe an observation study with a single centre experience of gravitational shunts in the treatment of pediatric hydrocephalus.

Material: During August 2008 - December 201578 gravitational ventriculo-peritoneal shunts were implanted (74 Pedi-GAV and 4 GAV valves, Christophe Miethke GMBH and Co KG, Potsdam, Germany) in 63 hydrocephalic children (27 males). New shunts were 42 of the 78 operations, 17 in the first year of life. Mean age at implantation was 51 months (range: 1.7-213). Mean followup was 38.2 months (range: 1-92). Cause of hydrocephalus was IVH in 19, tumour in 23, infection in 3, Dandy Walker syndrome in 2, vascular (AVM, vein of Galen) in 3 , congenital pathologies and aqueduct stenosis in 4, spina bifida in 2, and Chiari I in 2, arachnoid cyst in 3.

Results: Among the 78 implanted valve systems there were 14 obstructions (17.8\%), 3 infections (3.8\%), 2 underdrainage (previous CSF infection, the opening pressure of $4 \mathrm{~cm} \mathrm{H}_{2} \mathrm{O}$ was too high to be tolerated) and 1 case of overdrainage (slit ventricles, history of differential pressure as the first valve in the neonatal period). Of the 14 obstructions $6(7.6 \%)$ were due to ventricular catheter malposition (replaced in the first few days after surgery). Hence, the true valve system obstruction rate was $10.2 \%$. The total complication rate was $24.3 \%$ in mean follow up of 38 months.

Discussion: The midterm result of the use of gravitational valves in pediatric hydrocephalus is satisfactory, with a low early complication rate and no significant late side effects.

\section{PP92}

What shunts must be used in children with hydrocephalus? Dmitry Zinenko, Artyom Shramko, Evgenia Berdichevskaya, Maxim Vladimirov, Farhod Khafizov (Moscow, Russia)

Shunt surgery remains the most common treatment of pediatric Hydrocephalus, but it associated with big amount of complications and the most often and severe is overdrainage. Recent researches show that number of complications doesn't depend on design and pressure of the shunt.

Goal: To see how the flow through the shunt depends on the opening pressure and the effect of the length of the catheter to the stream of the CSF.

Method: We tested shunts of three companies for low, median, high an also adjustable shunts with simulation in vitro of an upright position ( $80 \mathrm{~cm}$ differential).

Results: All shunts opens according to figures in the manual information but after connection of standard distal catheter the flow becomes uncontrolled, independent to opening pressure and 10-50 higher than physiological volume of CSF production $(204-1200 \mathrm{ml} . \mathrm{p} / \mathrm{h})$.

Adjustable shunts showed flow 10 times smaller than shunts with fixed opening pressure $(36,6-55,2 \mathrm{ml} . \mathrm{p} / \mathrm{h})$.

Conclusion: Conventional differential pressure shunts are unphysiological and can't be suggested. Only adjustable shunts should be used in pediatric hydrocephalus. 